\title{
Viability Study of Photo-Voltaic Systems Added to Terrestrial Electric Vehicles
}

Wally E. Rippel

September 1990

Sucty carriod out under sponcorship of

U. S. Department of Energy

by

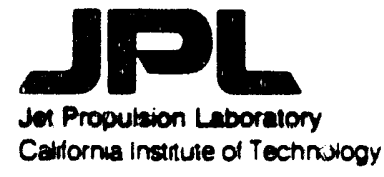


1. PURPOSE OF STUDY . . . . . . . . . . . . . . . . . . . . . . . $1-1$

2. BACKGROUND . . . . . . . . . . . . . . . . . . . . . . . . . 2 . 1

3. METHODOLOGY . . . . . . . . . . . . . . . . . . . . . . . . 3-1

4. COMPUTER PROGRAM . . . . . . . . . . . . . . . . . . . . . . . . 4-1

5. SOLAR INSOLATION MODEL . . . . . . . . . . . . . . . . . . . . . . 5-1

6. ARRAY MODEL . . . . . . . . . . . . . . . . . . . . . . . . $6-1$

6.1 ARRAY TYPES SELECTED FOR TABULATED RESULTS . . . . . . . . 6-1

6.2 TEMPERATURE CORRECTION OF EFFICIENCY . . . . . . . . . . . . 6-2

6.3 ARRAY WEIGHT . . . . . . . . . . . . . . . . . . . . . . 6-2

6.4 ARRAY AERODYNAMIC DEGRADATION . . . . . . . . . . . . . . 6-3

7. POWER CONVERTER MODEL . . . . . . . . . . . . . . . . . . . . . . . . 7-1

7.1 PARAMETERS . . . . . . . . . . . . . . . . . . . . $7-1$

8. VEHICLE ENERGY USE MODEL . . . . . . . . . . . . . . . . . . . . . . . 8-1

9. VEHICLE USE PATTERN . . . . . . . . . . . . . . . . . . . . . 9-1

10. BATTERY MODEL . . . . . . . . . . . . . . . . . . . . . . . . 10-1

10.1 LIFE MODELING . . . . . . . . . . . . . . . . . . . . . . 10-1

10.2 COST AND EFFICIENCY AND ENERGY STORAGE MODELLING . . . . 10-3

11. AN APPLICATION GUIDE TO APPENDIX A . . . . . . . . . . . . . . . . . 11-1

11.1 ESTIMATION OF PAYBACK TIME FOR CANDIDATE APPLICATIONS . • $11-2$

11.2 SENSITIVITY ANAIYSIS . . . . . . . . . . . . . . . . . 11-2

12. OBSERVATIONS . . . . . . . . . . . . . . . . . . . . . $12-1$

13. RECOMYENDATIONS FOR FUTURE WORK . . . . . . . . . . . . . . . . 13-1 REFERENCES . . . . . . . . . . . . . . . . . . . . . . . 13-3 
A

B

Eigures

4-1.

5-1.

5-2.

5-3.

5-4.

5-5.

5-6.

$5 \cdot 7$.

5-8.

9-1.

9-2.

$9-3$.

$9-4$.

9-5.

$10-1$.
PRINTOUT OF SIX PARAMETERS ASSOCIATED WITH

3240 PV-ASSIST SIMULATIONS . . . . . . . . . . . A-1

LISTING OF COMPUTER PROGRAM

(C LANGUAGE, FORMAT TAR)

B - 1

Data Processing Flow Chart . . . . . . . . . . . 4-2

Plot of Solar Insolation for Las Vegas . . . . . $5-4$

Plot of Solar Insolation for Bryce Canyon . . . . $5-4$

Plot of Solar Insolation for Sacramento... . . 5.5

Plot of Solar Insolation for Dallas-Fort Worth . 5-5

Piot of Solar Insolation for Atlanta . . . . . . 5-6

Plot of Solar Insolation for Detroit . . . . . . 5-6

Plot of Solar Insolation for Seattle . . . . . . 5-7

Plot of Solar Insolation for Fairbanks . . . . . 5-7

Plot of Vehicle Use Pattern No. $1 . . . . . . .99$

Plot of Vehicle Use Pattern No. 2........ 9.3

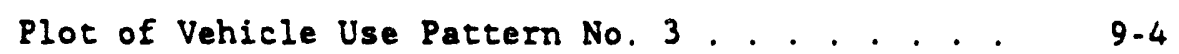

Plot of Vehicle Use Pattern No. 4. . . . . . . . 9-4

Plot of Vehicle Use Pattern No. 5. . . . . . . . 9.5

Battery Life Versus Depth of Discharge for Battery Types, 1,2 and 3 Using Constunts

Listed in Tabie $10-1$. . . . . . . . . . . . . . $10-4$ 


\section{Tables}

5-1. Iwolation Constants ................ 5-3

6-1. Array Parameters . . . . . . . . . . . . . . . . . . . . 6-1

7-1. Power Converter Constants . . . . . . . . . . . . 7-2

8-1. Vehicle Constants.................... 8-1

9-1. Vehicle Use Patterns . . . . . . . . . . . . . . . 9-2

10-1. Battery Life Constants . . . . . . . . . . . . . 10-3

10-2. Miscellaneous Battery Constants............. 10-5 


\section{SECTION 1}

PURPOSE OF STUDY

The purpose of the following computer study is to determine the set of necessary conditions under which the addition of photo-voltaic (PV) cells to electric vehicles provides a net utility or economic benefit. Economic benefits are given the primary focus and are evaluated in terms of a payback period. 
The potential benefits of adding PV cells to an electric vehicle (EV) include increased range, reduced recharge frequency, the possibility of independence from recharge power in some cases and reduced operating expense. The range benefit is generally questionable in that it epplies only to daytime driving and varies with location, weather, season and use patterns. Specifically, under worst-case conditions (e.g., night driving) the addition of PV will produce a slight range reduction due to added weight (and possibly increased air drag and parasitic power loss). If range is to be estimated under conservative conditions, then the range component of benefit will be negative. Section 6.3 provides a quantitative discussion of nightelme range reduction due to array weight.

While solar insolation is unpredictable at a given moment, over a long period, such as a year, the energy produced is moderately well defined. Accordingly, the long-term economic benefits of PV may be studied with some degree of certainty. The two main components of operating expense impacted by PV are electrical energy and battery replacement costs. As will be shown, reduced battery costs, due to increased life which results from reduced depth. of-discharge is often the major benefit of adding PV.

The economic benefit of adding PV is measured in terms of a payback period .. the time required for battery and energy cost savings to equal the cost of the array and associated hardware. It should be noted that this approach neglects the following considerations:

1. The cost of money (prevailing and future interest rates).

2. Long term trends in energy and battery replacement costs.

3. Long term trends in battery characteristics.

4. Maintenance costs of $\mathrm{PV}$ and associated hardware.

5. Indirect vehicle costs due to added weight of PV and associated hardware.

In the study, it is assumed that all utility type battery charging is done after sunset. It should be noted that daytime charging can be viewed as an alternative to $\mathrm{PV}$ for depth-of-discharge (DOD) reduction. Thus, in those cases, where on-vehicle PV produces substantial bettery life improvements, similar improvements could be achieved through daytine charging. This, however, would require the availability of utility power and power metering while prisenting the inconvenience of plugging and unplugging the vehicle. 
SECTION 3

METHODOLOGY

One way to evaluate the economic effectiveness of PV would be a single point evaluation dealing with an "average" EV operating under "average" weather and driving conditions using "average" PV hardware. This approach, however, is deficient in several ways:

1. In many cases, we do not know what "average" is.

2. It does not show which combination of circumstances are favorable and which are not.

3. It requires that all parameters and factors be identified at the outset.

A second approach (the one used in this study) is to let all parameters vary over a significant range. For each combination of parameters, the payback period is calculated and tabulated. With this approach, a detailed, functional view can be gained of where PV makes economic sense and where it does not. The user (not the author) selects the actual combination of array, battery, vehicle, vehicle use and vehicle location. To this end, six mathematical models are established and then linked together in a computer program. The individual models are:

1. Solar Insolation Model

This model provides a measure of solar radiation ( $W / \mathrm{m}^{2}$ ) through a horizontal surface as a function of location, season and time of day. Models for eight locations and four seasons are included. See Section 5 for detalls.

2. Array Model

Array efficiency and array cost are modelled for three different array types. Weight modeliing is not included. Sae Section 6 for details.

3. Power Converter Model

The switch-node converter which interfaces between the array and the battery bus 18 modelled with respect to efficiency and cost. Only one converter is modelled. See section 7 for detalls.

4. Vehicle Energy Use Model

This model provides energy use ( $\mathrm{kWh} / \mathrm{km}$ ) as a function of characteristic vehicle speed. Three different vehisle types are simulated. See Section 8 for detalls. 


\section{Vehicle Use Pattern Model}

This model defines vehicle speed versus time of day using simple step functions. Five use patterns are used in the simulation. See section 9 for details.

6. Battery Model

This model defines battery degradation as a function of battery parameters and DOD. Three battery types are handled in the simulation. See Section 10 for details.

In Section 4, the computer program which links the models is discussed; the computer print-out is included in Appendix $A$ and the program is listed in Appendix B.

Section 11 is dedicated to the use of the Appendix A results. Finally, Section 12 carries out observations and conclusions, while Section 13 identifies areas for future work. 
Figure 4-1 is a simplified flow diagram of the program. The purpose of this program is to determine payback periods for all combinations of the following:

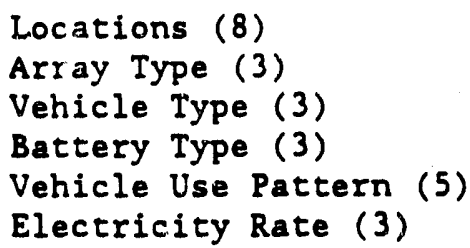

A total of $8 \times 3 \times 3 \times 3 \times 5 \times 3-3240$ cases are computed and tabulated. In addition, for each case, the following are also computed and tabulated:

1. Up-Front Costs

(This is the sum of the array and converter plus installation costs.)

2. Percent Energy Reduction

(This is the fractional decrease in recharge energy due to the addition of PV.)

3. Maximum Winter Deptil of Discharge

(This is the maximum depth of discharge where zero PV energy is assumed. )

4. Percent DOD Reduction

(This is the yearly average reduction of DOD due to the inclusion of PV.)

\section{Percent Battery Life Increase}

(This is the fraction by which batcery 11fe is increased due to the inclusion of $\mathrm{PV}$. )

Referring to the flow diagram of Figure 4-1, blocks 1 through 6 provide an output of power into the battery bus varsus time of day and season for each of the eight selected locations. If the battery is fully charged, none of this power is applied to the battery. 


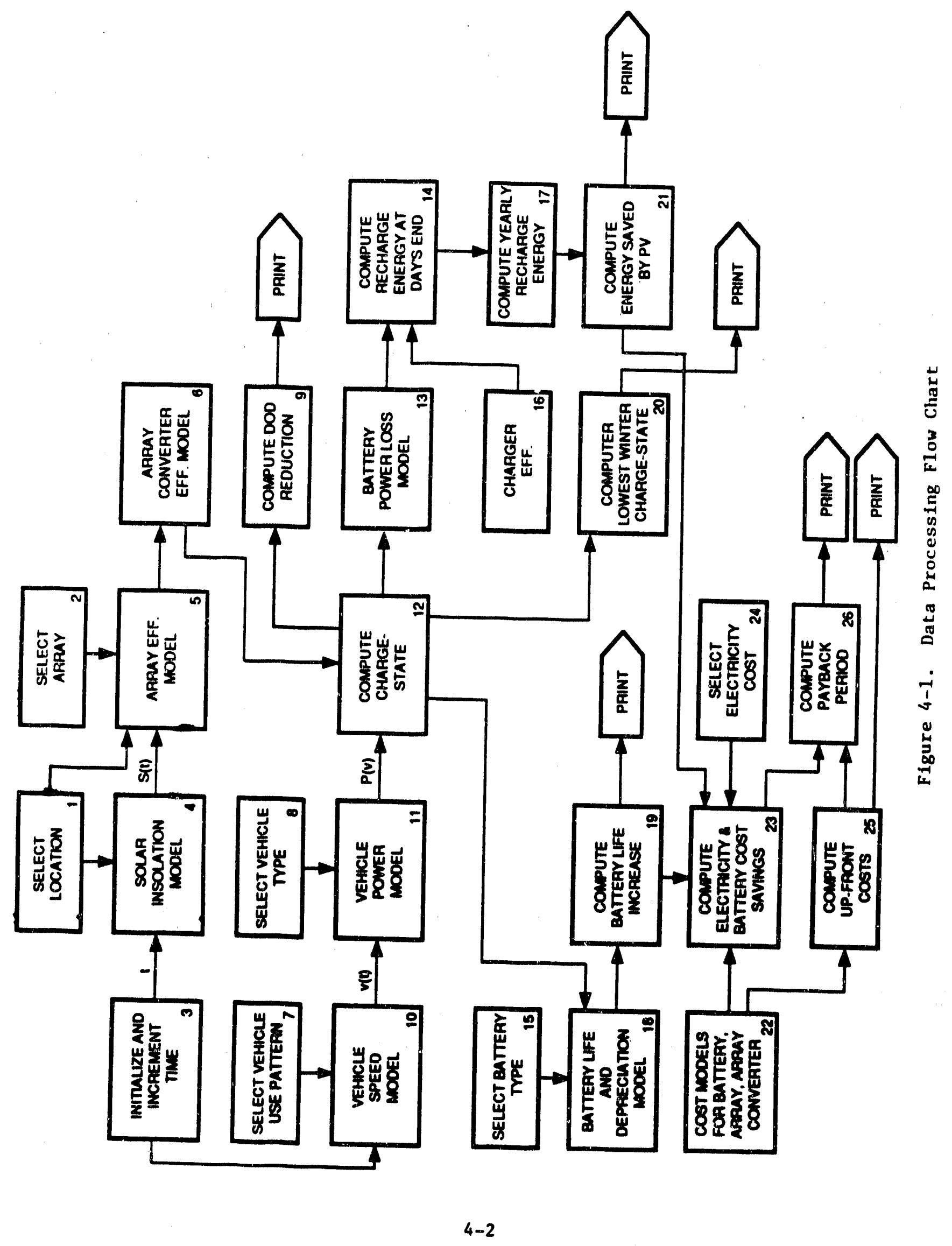


Blocks 3, 7, 8, 10 and 11 provide an output of power from the battery versus time of day, vehicle type and vehicle use pattern. Block 12 integrates the difference of recharge and discharge power to determine charge state. At the start of each day, full charge (08 DOD) is assumed. During the day, the point of maximum discharge is selected and used for calculation of battery depreciation.

Blocks 13, 14, 16 and 17 provide computation of yearly recharge energy. This calculation is performed twice -. once without PV and once with the PV output of block 6 .

Using daily maximum DOD (from block 12), blocks 15, 18 and 19 compute battery depreciation rate and life for both the cases without and with PV. Blocks 22 and 25 serve to compute the up-front costs associated with the PV addition.

Blocks 23 and 24 serve to compute yearly electricity cost and battery depreciation for the cases without and with PV. Finally, block 26 uses the outputs of blocks 23 and 25 to compute the payback period. (Note that no account is given for the cost of money; i.e., a zero interest rate is as sumed.) 
SOLAR INSOIATION MODEL

As discussed in Section 4, the purpose of this model is to provide estimates of solar insolation, $S(t)\left(W / \mathrm{m}^{2}\right.$ on a horizontal surface) as a function of location, season and time of day. Data are obtained from Ref. 1 which provide total solar energy per horizontal square meter per day as a function of season and location for a so-called "Typical Meteorological Year" (TMY). Using this data and trigonometric considerations, $S(t)$ was obtained for eight US locations for each of the four seasons.

If $S_{0}$ is the solar flux through a surface which is normal to the sun's direction, then, by noting the dot product of the sun vector and a vector normal to the Earth's surface, the insolation through a horizontal surface is found for the summer and winter solstices as:

$$
S(t)=S_{0}(\cos \alpha \cos \theta \cos \omega t+\sin \alpha \sin \theta) \text {, }
$$

where $\alpha$ is the Earth's inclination relative to the ecliptic plane ( $\alpha$ - 0.4084 radians for summer solstice; $\alpha-0.4084$ radians for winter solstice), $\theta$ is the latitude of the location in question, $w$ is the Earth's rotation rate $(0.2618 \mathrm{radian} / \mathrm{hour})$ and $t$ is the elapsed time in hours after high noon.

At sunset, $S(t)=0$. Accordingly, Equation (5-1) gives solstice sunset times as

$$
T_{s}-\frac{1}{\omega} \cos ^{-1}[-\tan \alpha \tan \theta]
$$

The average spring and summer sunset times $(T)$ are taken as a weighted average of the equinox and solstice sunset times:

$$
T=\frac{1}{3} T_{E}+\frac{2}{3} T_{S}
$$

As $T_{E}=6.00$ hours,

$$
T=2.000+2.546 \cos ^{-1}(\mp 0.4328 \tan \theta)
$$

(The minus sign applies for spring and summer and the plus sign applies for Eall and winter.)

Equation (5-1) may be written in tise form:

$$
S_{i j}(t)=A_{i j} \cos \omega t+B_{i j} \text {. }
$$

where $i$ refers to location and $j$ refer to season. Integrating $(5-5)$ over $t$ from sunrise to sunset glves the total energy per day, $E_{i j}$. In turn, solving for $A_{i j}$ and $B_{i j}$ gives: 


$$
\begin{aligned}
& A_{i j}=\frac{1}{2} E_{i j} /\left(\frac{1}{\omega} \sin \omega T_{i j}-T_{i j} \cos \omega T_{i j}\right) \\
& B_{i j}=-A_{i j} \cos \omega T_{i j}
\end{aligned}
$$

Values of $F_{i j}, \theta_{i}, T_{i j}, A_{i j}$ and $B_{i j}$ are listed in Table 5-1 for each of eight US locations. $E_{i j}$ and $\theta_{i}$ data are obtained from Ref. 1 . Actual locations chosen are such that consecutivi $E_{i}$ increments are approximately equal. Plots of Eqn. (5-5) for each of the elght locations a:s shown in Figures 5-1 through 5-8. Eqn. (5-5) and computer generated villues of $A_{i j}$ and $B_{i j}$ are inputs to the simulation projram. 


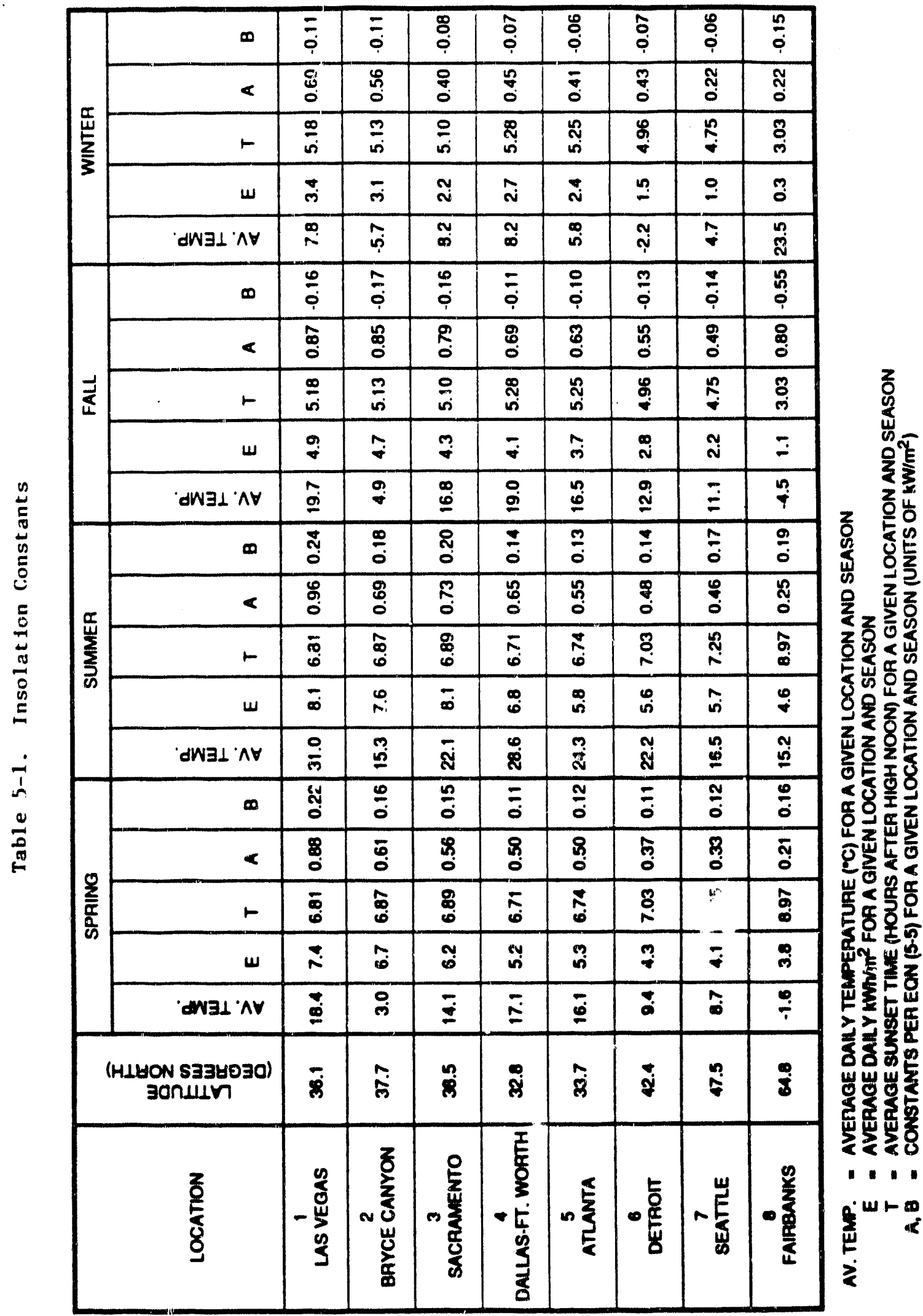




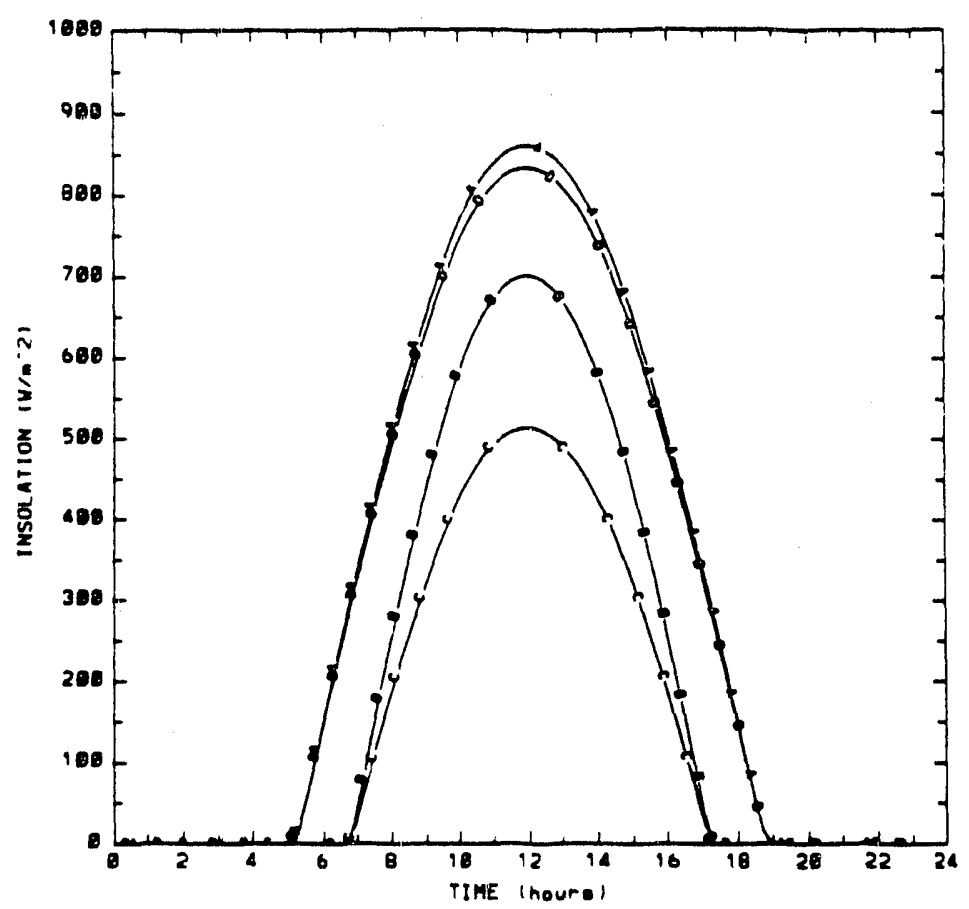

Figure 5-1. Plot of Solar Insolation for Las Vegas. Averages for Sumer (A), Fall (B), Winter (C) and Spring (D)

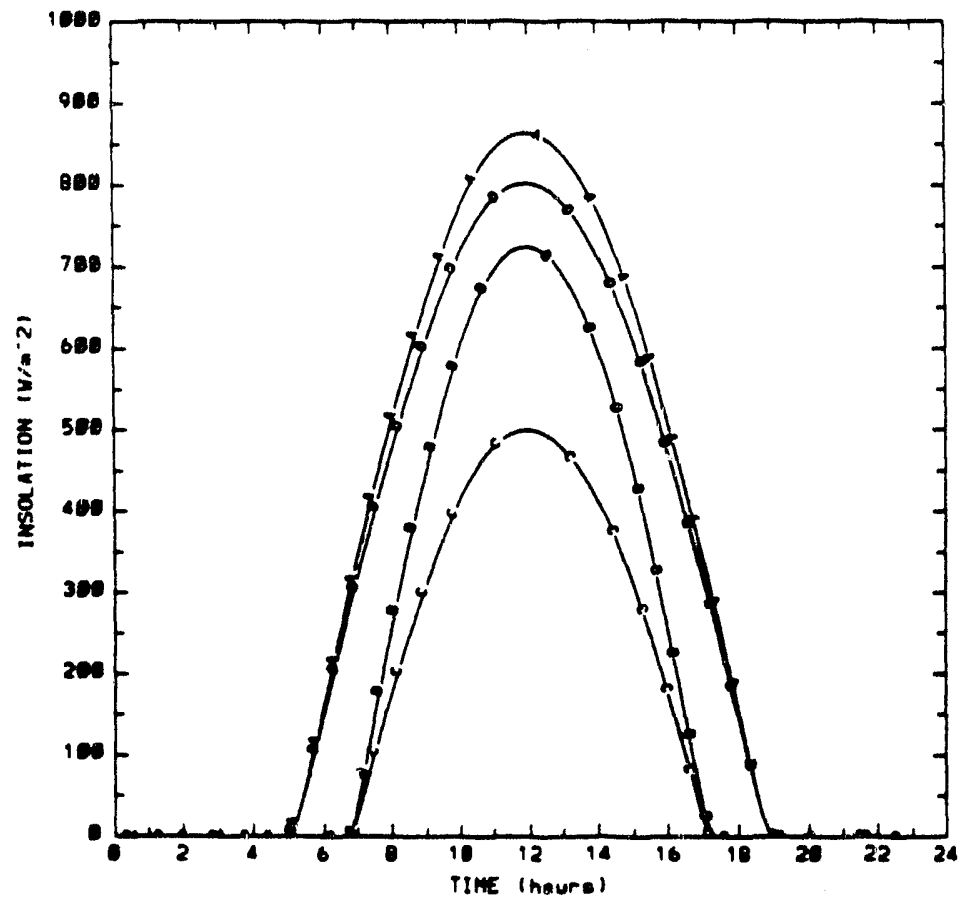

Figure 5-2. Plot of Solar Insolation for Bryce Canyon. Averages for Sumer (A), Fall (B), Wintex (C), and Spring (D) 


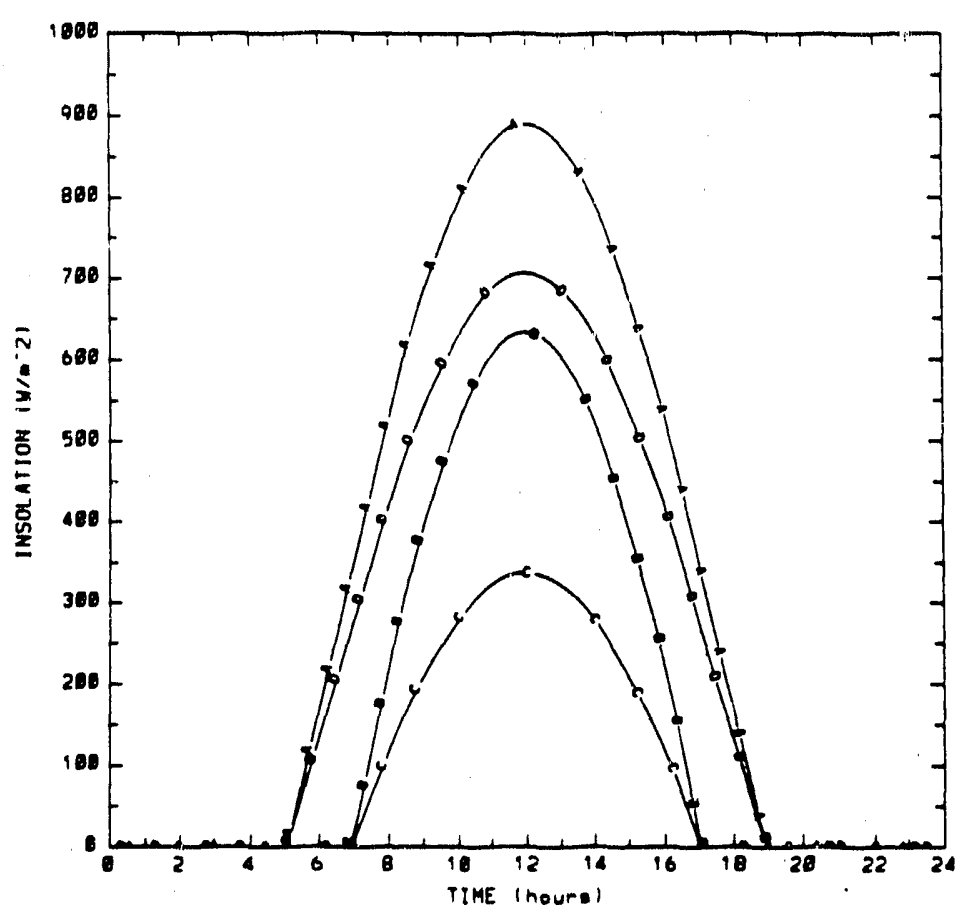

Figure 5-3. Plot of Solar Insolation for Sacramento. Averages for Summer (A), Fall (B), winter (C) and Spring (D)

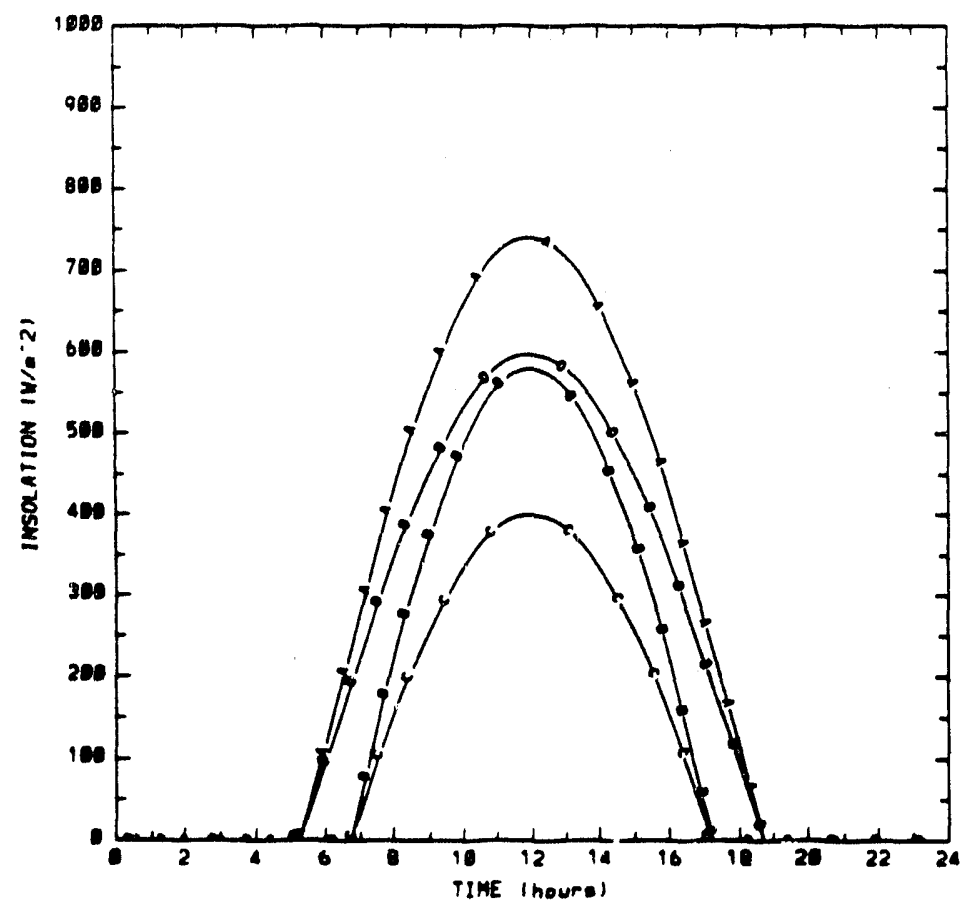

Figure 5-4. Plot of Solar Insolation for Dallas-Fort Worth, Averages for Sumer (A), Fall (B), Winter (C) and Spring (D) 


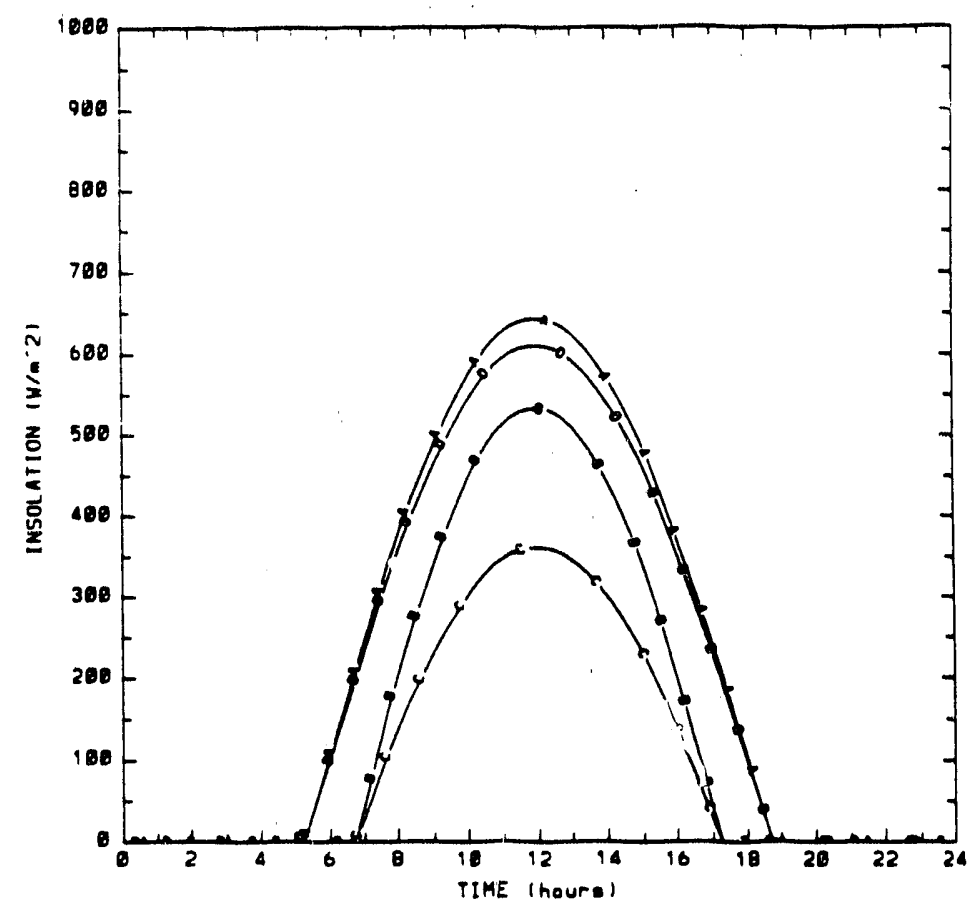

Figure 5-5. Plot of Solar Insolation for At lanta. Averages for Summer ( $A_{A}$ ), Fall (B), winter (C) and Spring (D)

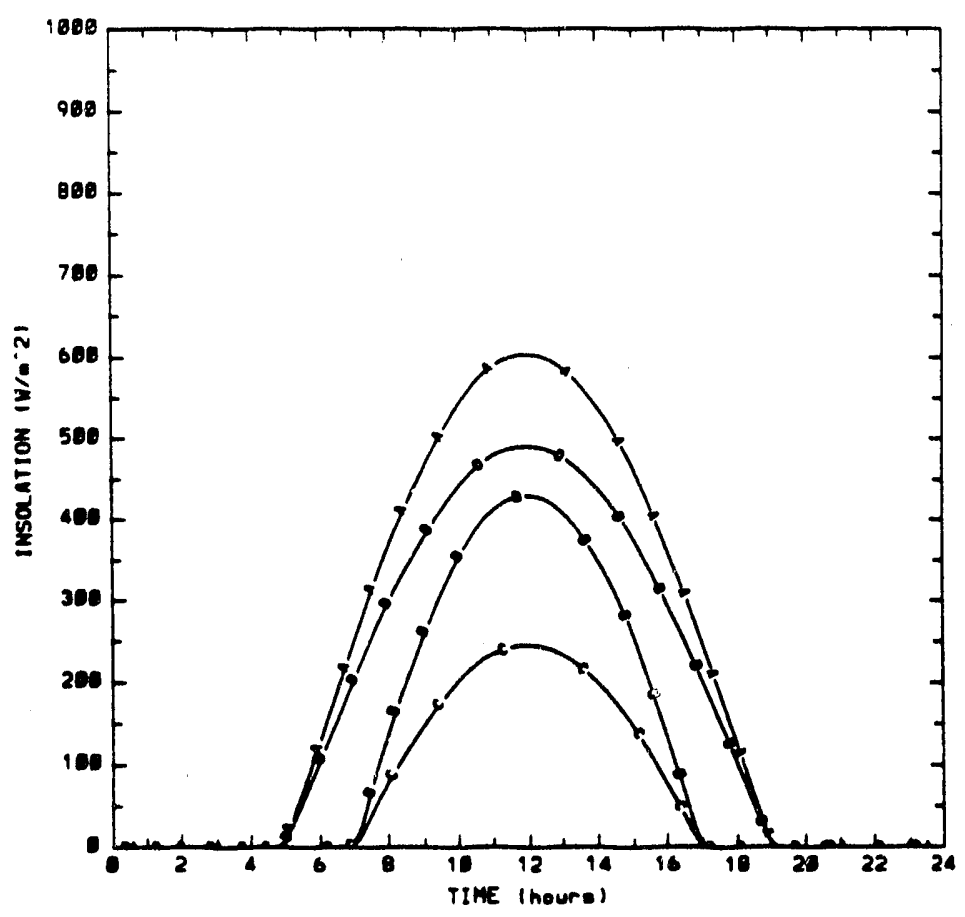

Figure 5-6. Plot of Solar Insolation for Detrolt. Averdges for Summer (A), Fall (B), Winter (C) and SpCIng (D) 


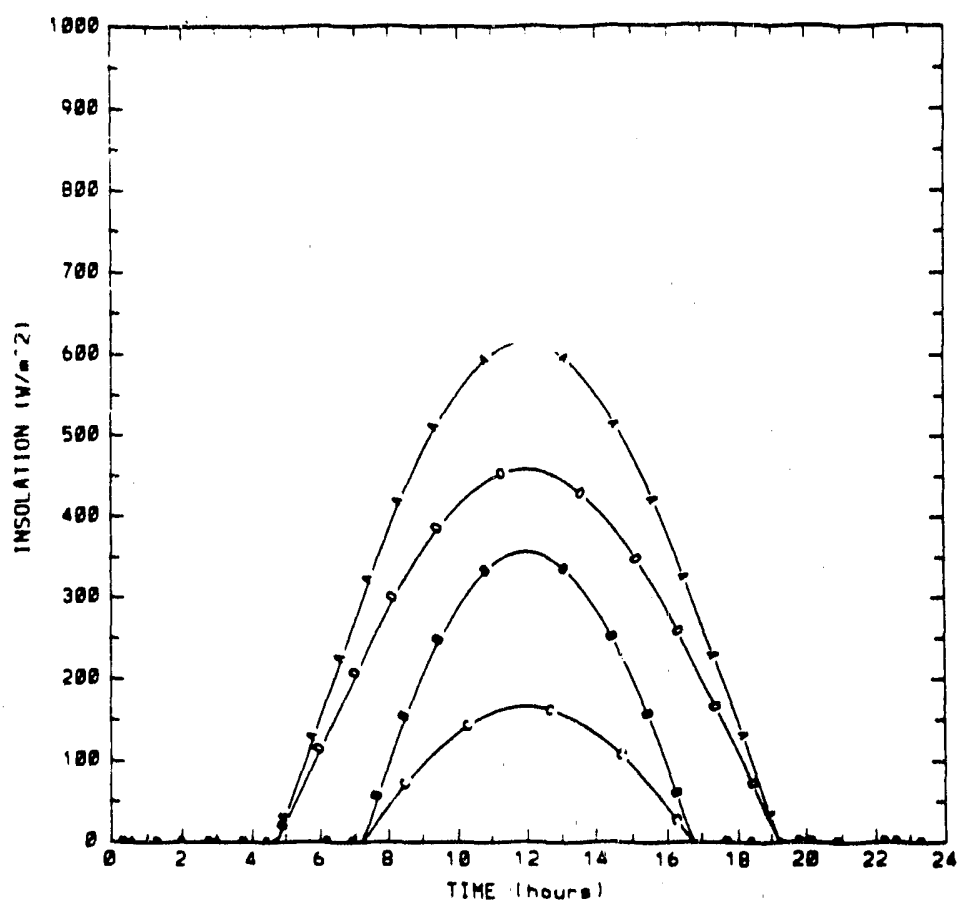

Figure 5-7. Plot of Solar Insolation for Seattle. Averages for Summer (A), Fall (B), Winter (C) and Spring (D)

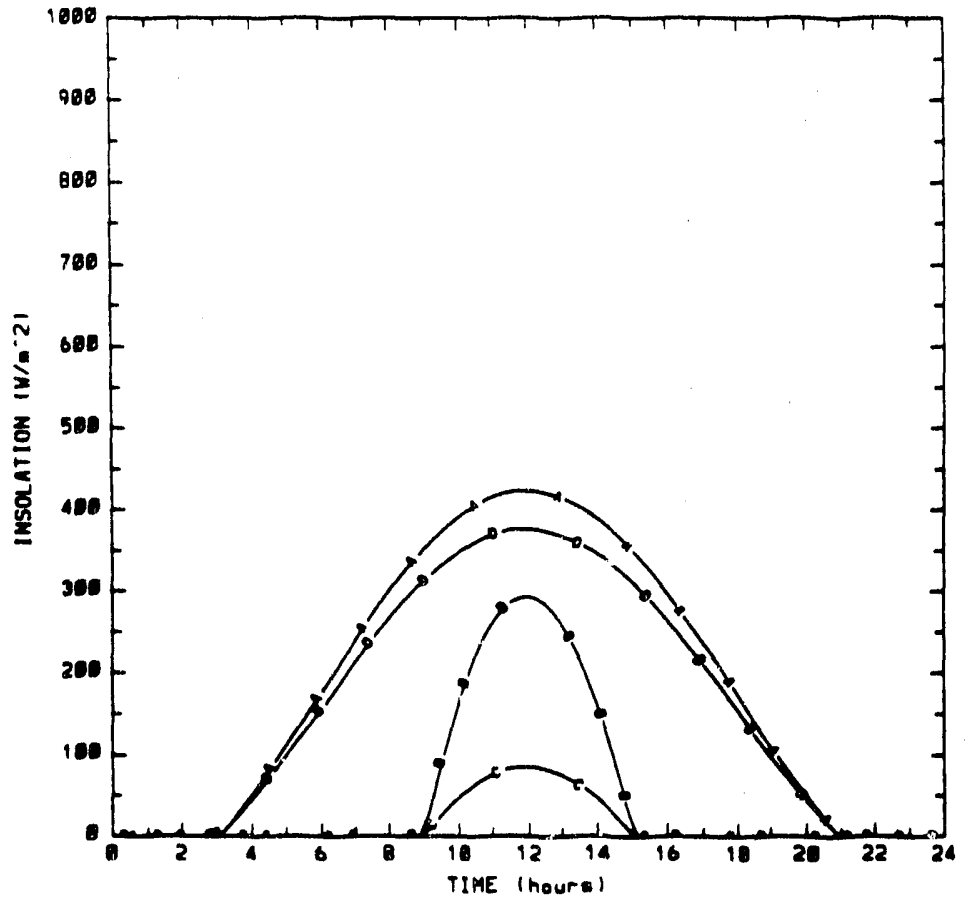

Figure 5-8. Plot of Solar Insolation for Falrbanks. Averages for Sumer (A), Fall (B), Winter (C) and Spring (D) 
SECTION 6

ARRAY MODEL

The purpose of the array model is to provide estimates of array efficiency, cost, weight and aerodynamic degradation for candidate array types. Several issues are recognized, but not given account in the simulation due to lack of available data. These include:

1. Efficiency degradation due to aging.

2. Efficiency degradation due to build up of foreign matter.

3. Efficiency degradation due to temperature rise above ambient (which in turn is a function of vehicle speed-time profile and array construction).

4. Efficiency degradation due to shadowing effects -. both while driving and while parked.

Because of the above effects which diminish efficiency and the difficulty in properly quantifying these effects, it was decided that array efficiency be defined on an in sit's basis. As such, the above issues must be given account by the user in determining a derated nominal efficiency. This derated efficiency can then be used in the simulation program. Where Appendix A tabulated data is used, data blocks should be selected having array parameters which best approximate the array in question.

\section{1}

\section{ARRAY TYPES SELECTED FOR TABUIATED RESULTS}

Three type of arrays were selected for the simulation. For each array type, two cost components are considered .. a fixed cost (C1) associated with installation and a proportional cost (C2) which is proportional to the array area (A). Array efficiency (N1) is in situ. Accordingly, corresponding stand-alone efficiencies must be appropriately higher. Cost and efficiency parameters are listed in Table 6-1 for the three array types. (In all cases, the array area is $2 \mathrm{~m}^{2}$.)

Table 6-1. Array Parameters

\begin{tabular}{|l|c|c|c|}
\hline & Type 1 & Type 2 & Type 3 \\
\hline In situ Efficiency (N1) & 0.08 & 0.25 & 0.10 \\
\hline Installation Cost* (C1) & $\$ 150$ & $\$ 250$ & $\$ 250$ \\
\hline Array Cost $/ \mathrm{m}^{2 *}$ (C2) & $\$ 150$ & $\$ 500$ & $\$ 1000$ \\
\hline Array Area Assumed (A) & $2 \mathrm{~m}^{2}$ & $2 \mathrm{~m}^{2}$ & $2 \mathrm{~m}^{2}$ \\
\hline
\end{tabular}

* Cost at retail level, 1990 dollars 
Array type 1 represents present single junction silicon technology which is in production. Assuming a nominal stand-alone efficiency of 10 and a peak insolation of $1000 \mathrm{~W} / \mathrm{m}^{2}$, cost per peak dc watt is $\$ 1.50$, excluding installation costs.

Array type 2 represents a future possibility of very high efficiency made possible by multi-junction techniques; the corresponding stand-alone efficiency would be on the order of 308 to 358 . The cost of $\$ 500 / \mathrm{m}^{2}$ which again corresponds to about $\$ 1.50$ per peak watt is optimistic in that high efficiency technology is expected to have considerably higher cost per watt cost when compared with "conventional efficiency" technology. Note that assumed installation cost for type 2 is higher than for type 1 ; this is based on the assumption that type 2 technology will be produced in lower: volume and may be more sensitive to mechanical stress.

Array type 3 represents conventional single junction silicon technology selected for high efficiency. Like type 1, type 3 technolog. is presently in production. The high cost of $\$ 8.00$ per watt is consistent with limited volume and relatively high efficiency (12 to 15 stand alone).

\subsection{TEMPERATURE CORRECTION OF EFFICIENCY}

For a given array installation, array temperature will tend to track with ambient temperature. Accordingly, efficiency may be corrected in accordance with the average dally temperature for a given location and season. From Ref. 4, the temperature corrected efficiency, $E(T)$ is given as

$$
E(T)=E_{0}(1.121-0.00433 T) \text {, }
$$

where $E_{0}$ is the nominal efficiency at 28 degrees $C$ and $T$ is the array temperacure (degrees $C$ ). In the simulation program, Eqn. $(6-1)$ was applied to each of the three array types for each location and for each season, using average daily temperature for the value of $T$. While this approach suffers from several errors, it is expected to give approximately correct relative results between the different locations.

\subsection{ARRAY WEIGHT}

The addition of the array adds welght which is proportionate to array area. The added weight is due mainly to support structure and protective covering. Based on experience with experimental vehicles, the added weight factor appears to be in the range of 5 io $10 \mathrm{~kg} / \mathrm{m}^{2}$ with present technology. In that electric vehicles typically have an energy versus weight sensitivity of about 0.5 , depending on the type of driving. It follows that a $1000 \mathrm{~kg}$ vehicle will require 0.25 to 0.50 nore energy per square meter of array. Accordingly, the nighttime range will be reduced by a similar percentage. In that typical array outputs are on the order of $100 \mathrm{~W} / \mathrm{m}^{2}$ for peak sunlight, while typical vehicle propulsion power levels are on the order of $10 \mathrm{~kW}$, it follows that the net range increase during daytine will be on the order of one percent per square meter of array. Assuming 30 array efficiencies are possible in the future and assuming that very high-efficiency 
vehicle designs are also used $(60 \mathrm{~W}-\mathrm{hr} / \mathrm{km})$, the range benefit could reach 5 per square meter for highway driving and 10 or or more per square meter for city driving .. under ideal sun conditions.

On the other hand, it should also be noted that either the vehicle payload must be reduced to accommodate the added weight of the array or vehicle structure must be added. Finally, if acceleration and range are to be preserved when PV is added, then added propulsion and corresponding structure mass are required .. which, in turn, add cost and increase the energy use. From previous experiences with electric vehicles, the above weight and cost multipliers are on the order of $1.4 \mathrm{~kg} / \mathrm{kg}$ and $\$ 7.00 / \mathrm{kg}$. Thus, with present technology, adding one square meter of array means that the net vehicle mass will increase from $7 \mathrm{~kg}$ to $14 \mathrm{~kg}$ while the vehicle cost (excluding the array) will increase from $\$ 50$ to $\$ 100$.

In the simulation, no account was given fti array weight, due both to the lack of solid data and the belief that very light weight structures (less than $1 \mathrm{~kg} / \mathrm{m}^{2}$ ) may be realistic in the near future. In any case, the simulation is "optimistic" with respect to the issue of array weight.

\section{4}

\section{ARRAY AERODYNAMIC DEGRADATION}

If the array is carefully integrated with the vehicle body, the increase in aerodynamic drag $\left(C_{D} A\right)$ may be negligible. On the other hand, if the array is not integrated with body, as in the case where a flat, non-curved array is used, a significant aerodynamic penalty will occur.

For the simulation, it was assumed that the array would be fully integrated with the vehicle body and that no increase in $C_{D} A$ would result. Accordingly, the simulation may be optimistic in this respect. 


\section{SEC'TION 7}

POWER CONVERTER MODEL

The Power converter (PC) provides an interface between the solar array and the vehicle battery bus. The role of the PC is to present a load line to the solar array such that maximum array power is extracted and applied to the battery bus .. except in cases where battery charge acceptance is limited due to either a high charge state or due to the pressure of recharge power provided by regenerative braking.

Hardware consists of two main circuit blocks .. a power section and a control section. The power section performs the role of a variable ratio de transformer while the control section provides a control signal to the power section in response to sensed inputs such 8 s array voltage and current and battery voltage. Typically, the power section is a switch-mode buck regulator; this configuration requires that the array voltage be greater than the battery voltage. Alternatively, boost regulator may be used; this requires that the array voltage be less than the battery voltage. The boost scheme is favored in the case of high voltage battery systems in that lower (and safer) array voltage is utilized. Both the buck and boost schemes have negative grounds which are common to input and output.

The control section is essentially a function generator .. which may be implemented by either analog or digital weans. Various algorithms and circuit techniques have been devised which provide the desired control results.

\subsection{PARAMETERS}

Parameters relevant to the simulation include efficiency, weight and cost. In turn, efficiency is modelled in terms of a "fixed" or "tare" loss (PO) combined with an incremental efficiency (N2).

Control circuit power, magnetic core losses and semiconductor capacitance are physically responsible for the tare loss component while switching and resistive losses are physically responsible for the incremental term. Based on experience with state-of-the-art hardware, the tare loss is estimated at $1.6 \mathrm{~W}$ and the incremental efficiency is estimated at $98 \mathrm{*}$. No losses are assumed due to imperfections in the control circuit (1.e., a 100 tracking efficiency is assumed).

Based on demonstrated hardware, power to welght ratios of better than $1 \mathrm{~kW} / \mathrm{kg}$ are now avallable. Accordingly, for a high efficiency (258) array, converter mass will not exceed 250 \& per square meter of array. Because of the small mass impact, no account was given in the simulation.

Cost may be resolved into two components -. one fixed (C3) and one which is proportionate to the converter power rating ( $\mathrm{C} 4$ is proportionality constant). The fixed cost terw relates mainly to the controls .. which are not impacted by power level. Conversely, the proportional term relates to the 
power section where cost and mass are nearly proportionate to power level. A fixed cost of $\$ 25.00$ was assumed based on experience with state-of-the-art hardware. Likewise, a cost of $\$ 300 / \mathrm{kW}$ was assumed for the power section. (Note that the converter input power is equal to NIA, where NI is the array efficiency and $A$ is the array area.) Constants for the converter are summarized in Table 7-1.

In the simulation, $P O$ is subtracted from the available array power, and $\mathrm{N} 2$ times the remaining power is applied to the battery bus unless the battery is fully charged .. in which case, the converter output power is assumed zero.

Table 7-1. Power Converter Constants

\begin{tabular}{|l|c|}
\hline Tare Loss (PO) & $1.6 \mathrm{~W}$ \\
\hline Incr. Eff. (N2) & 988 \\
\hline Cost Intercept* (C3) & $\$ 25$ \\
\hline Cost Coefficient* (C4) & $\$ 300 / \mathrm{kW}$ \\
\hline
\end{tabular}

*Cost at retail level, 1990 dollars 


\section{SECTION 8}

VEHICLE ENERGY USE MODEL

The purpose of this model is to provide measures of energy use (measured at the battery bus) for candidate vehicles as a function of speed. In order to simplify both the vehicle energy use and vehicle use pattern models, it is assumed that the number of starts and stops per km varies inversely with the characteristic driving speed; one start/stop per $\mathrm{km}$ is assumed at $40 \mathrm{~km} / \mathrm{hr}$. With the assumption, energy use per $\mathrm{km}$ may be expressed as

$$
E_{B}-E_{o}+A v+B v^{2},
$$

where $E_{B}$ is the energy in $W-h$ removed from the battery bus per km of driving, $U$ is the steady-state speed and $E_{O}, A$ and $B$ are constants which characterize the energy use per $\mathrm{km}$ for a given vehicle. It should be noted that $E_{B}$ represents the sum of all losses associated with tires, aerodynamics and the drive system (excluding the battery aud battery charger; these losses are separately handled).

In the simulation, three vehicle types were considered. The first is a small four-seat passenger vehicle designed specifically for high efficiency and low weight. Vahicle curb weight is $1200 \mathrm{~kg}$ and energy use, $E_{B}$, at $97 \mathrm{~km} / \mathrm{hr}(60 \mathrm{mph})$ is $8900 \mathrm{~W}$. The second vehicle type represents a typical four-seat conversion - - characterized by $1400 \mathrm{~kg}$ and an energy use of $18 \mathrm{~kW}$ at $97 \mathrm{~km} / \mathrm{hr}$. The third vehicle type represents a van conversion having a curb weight of $2300 \mathrm{~kg}$ and an energy use of $30 \mathrm{~kW}$ at $97 \mathrm{~km} / \mathrm{hr}$.

Constants for the three vehicle types are summinized in Table 8-1.

Table 8-1. Vehicle Constants

\begin{tabular}{|l|c|c|c|}
\hline & Type 1 & Type 2 & Type 3 \\
\hline Vehicle Curb Weight (kg) & 1200 & 1400 & 2300 \\
\hline Wh/km @ 97 km/hr. & 92 & 182 & 310 \\
\hline$E_{\rho}(\mathrm{Wh} / \mathrm{km})$ & 30 & 55 & 100 \\
\hline A $\left(\mathrm{Wh}^{2} / \mathrm{cm}^{2}\right)$ & 0.21 & 0.45 & 0.71 \\
\hline B $\left(\mathrm{wh}^{3} / \mathrm{km}^{3}\right)$ & 0.0045 & 0.0090 & 0.0150 \\
\hline
\end{tabular}


A vehicle use pattern is defined by a speed-time function. "Realworld" use patterns are complex in that the use pattern is a function of the individual driver, his specific needs, the vehicle type, the vehicle age, the time of year, etc. Accordingly, idealized and simplified driving cycles or use patterns are sought .. which capture the important parameters such as total mileage per year and speed versus time of day. Using data from Ref. 2, a total of five ise parterns were defined. Each use pattern is characterized by a simple step function which repeats for each day of the year. Each of the five use patterns is defined in Table 9.1 and is shown graphically in the plots of Figures 9-1 through 9-5.

Use patterns one, two and three are associated with commuter driving to and from a place of work. Time of arrival at the work place is $8 \mathrm{a} . \mathrm{m}$. and time of departure is 5 p.m. Each day, at noon, a short trip is made.

With pattern one, the one-way commute distance is $10 \mathrm{~km}(6 \mathrm{mi})$ at an average speed of $40 \mathrm{~km} / \mathrm{hr}$ (24 mph). During lunch, a short drive of $5 \mathrm{~km}$ ( $3 \mathrm{mi}$ ) at $30 \mathrm{~km} / \mathrm{hr}(18 \mathrm{mph})$ is taken. Use patterns two and three are similar to pattern one except that commute speeds and durations are increased.

Use patterns four and five are associated with random daily commuter driving. These use patterns characterize use by such as unemployed parents or delivery services; use is evenly distributed through the day. With pattern four, use is 1 ight and total yearly driving is $9125 \mathrm{~km}$ (5700 mi) and with pattern five, use is heavy; yearly use is $27,375 \mathrm{~km}(17,100 \mathrm{mi})$.

It should be noted that above use patterns a:e "simplistic" in that the following items are missing:

1. Night driving.

2. Variations with seasons.

3. Variations with weather.

4. "High Frequency" details of speed versus time.

Despite these limitations, it is belleved that the five patterns are useful and give meaningful "first-order" results. 
Table 9-1. Vehicle Use Patterns

\begin{tabular}{|c|c|c|c|c|c|c|c|c|}
\hline $\begin{array}{l}\text { START } \\
\text { TIME(S) } \\
\text { (hr) }\end{array}$ & $\begin{array}{l}\text { AVERAGE } \\
\text { SPEED } \\
\text { (km/hr) }\end{array}$ & $\begin{array}{c}\text { FINISH } \\
\text { TIME(S) } \\
(h r)\end{array}$ & $\begin{array}{l}\text { START } \\
\text { TIME(S) } \\
\text { (hr) }\end{array}$ & $\begin{array}{l}\text { AVERAGE } \\
\text { SPEED } \\
\text { (km/hr) }\end{array}$ & $\begin{array}{l}\text { FINISH } \\
\text { TIME(S) } \\
\text { (hr) }\end{array}$ & $\begin{array}{l}\text { START } \\
\text { TIME(S) } \\
\text { (hr) }\end{array}$ & $\begin{array}{l}\text { AVERAGE } \\
\text { SPEED } \\
\text { (km/hr) }\end{array}$ & $\begin{array}{c}\text { FINISH } \\
\text { TIME(S) } \\
\text { (hr) }\end{array}$ \\
\hline \multicolumn{9}{|c|}{$\begin{array}{c}\text { USE PATTERN NO. } 1 \\
\text { LOW USE DAILY COMMUTE (25 km day, } 9125 \mathrm{~km} / \mathrm{yr})\end{array}$} \\
\hline-4.25 & 40 & .4 .00 & 0.00 & 30 & 0.17 & 5.00 & 40 & 5.25 \\
\hline \multicolumn{9}{|c|}{$\begin{array}{c}\text { USE PATTERN NO. } 2 \\
\text { MODERATE USE DAILY COMMUTE (50 km/day, } 18,250 \mathrm{~km} / \mathrm{yr})\end{array}$} \\
\hline-4.33 & 60 & -4.00 & 0.00 & 30 & 0.33 & 5.00 & 60 & 5.33 \\
\hline \multicolumn{9}{|c|}{$\begin{array}{l}\text { USE PATTERN NO. } 3 \\
\text { HIGH USE DAILY COMMUTE (75 km/day } 27,375 \mathrm{~km} / \mathrm{yr})\end{array}$} \\
\hline-4.38 & 80 & -4.00 & 0.00 & 30 & 0.50 & 5.00 & 80 & 5.50 \\
\hline \multicolumn{9}{|c|}{$\begin{array}{l}\text { USE PATTERN NO. } 4 \\
\text { LOW USE RANDOM COMMUTE (25 km/day, } 9125 \mathrm{~km} / \mathrm{yr} \text { ) }\end{array}$} \\
\hline $\begin{array}{r}-5.00 \\
1.00 \\
7.00\end{array}$ & $\begin{array}{l}40 \\
40 \\
40\end{array}$ & $\begin{array}{r}-4.90 \\
1.10 \\
7.03\end{array}$ & $\begin{array}{r}-3.00 \\
3.00\end{array}$ & $\begin{array}{l}40 \\
40\end{array}$ & $\begin{array}{r}-2.90 \\
3.10\end{array}$ & $\begin{array}{r}-1.00 \\
5.00\end{array}$ & $\begin{array}{l}40 \\
40\end{array}$ & $\begin{array}{r}-0.90 \\
5.10\end{array}$ \\
\hline \multicolumn{9}{|c|}{$\begin{array}{c}\text { USE PATTERN NO. } 5 \\
\text { HIGH USE RANDOM COMMUTE (75 km/ay, } 27,375 \mathrm{~km} / \mathrm{y})\end{array}$} \\
\hline $\begin{array}{r}-5.00 \\
1.00 \\
7.00\end{array}$ & $\begin{array}{l}60 \\
60 \\
60\end{array}$ & $\begin{array}{r}4.80 \\
1.20 \\
7.05\end{array}$ & $\begin{array}{r}-3.00 \\
3.00\end{array}$ & $\begin{array}{l}60 \\
60\end{array}$ & $\begin{array}{r}-2.80 \\
3.20\end{array}$ & $\begin{array}{r}-1.00 \\
5.00\end{array}$ & $\begin{array}{l}60 \\
50\end{array}$ & $\begin{array}{r}-0.80 \\
5.20\end{array}$ \\
\hline
\end{tabular}




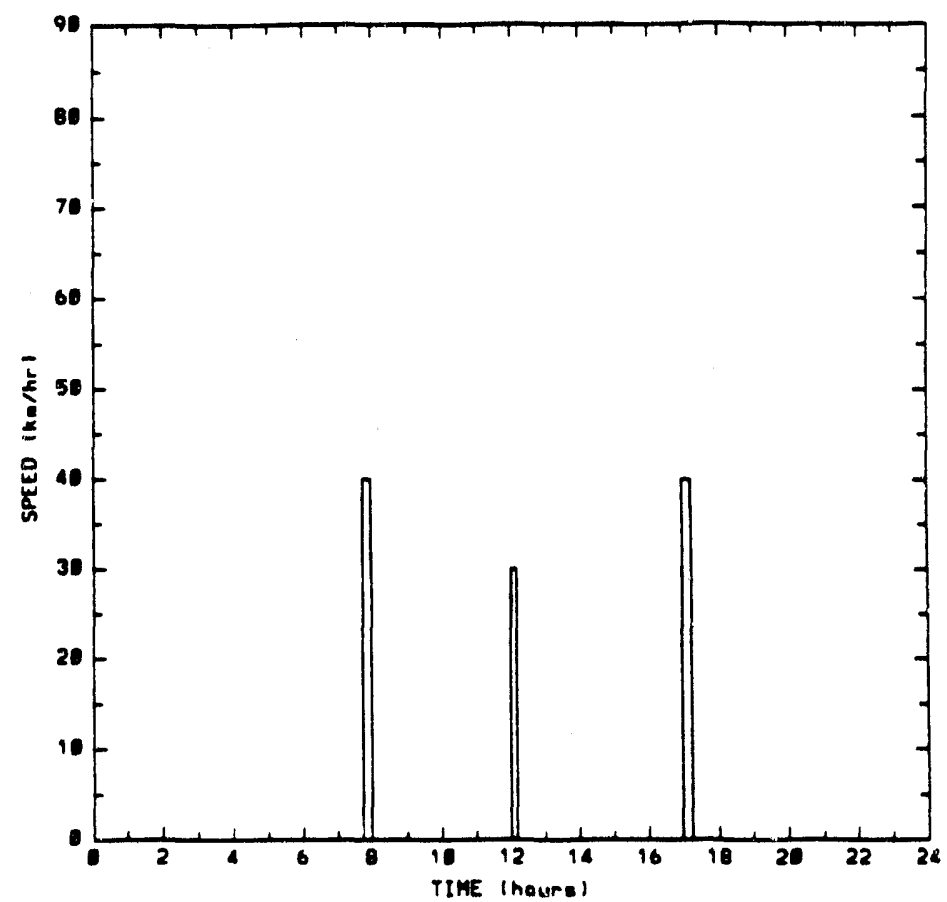

Figure 9-1. Plot of Vehicle Use Pattern

No. 1 (Time of Day Starts at Midnight)

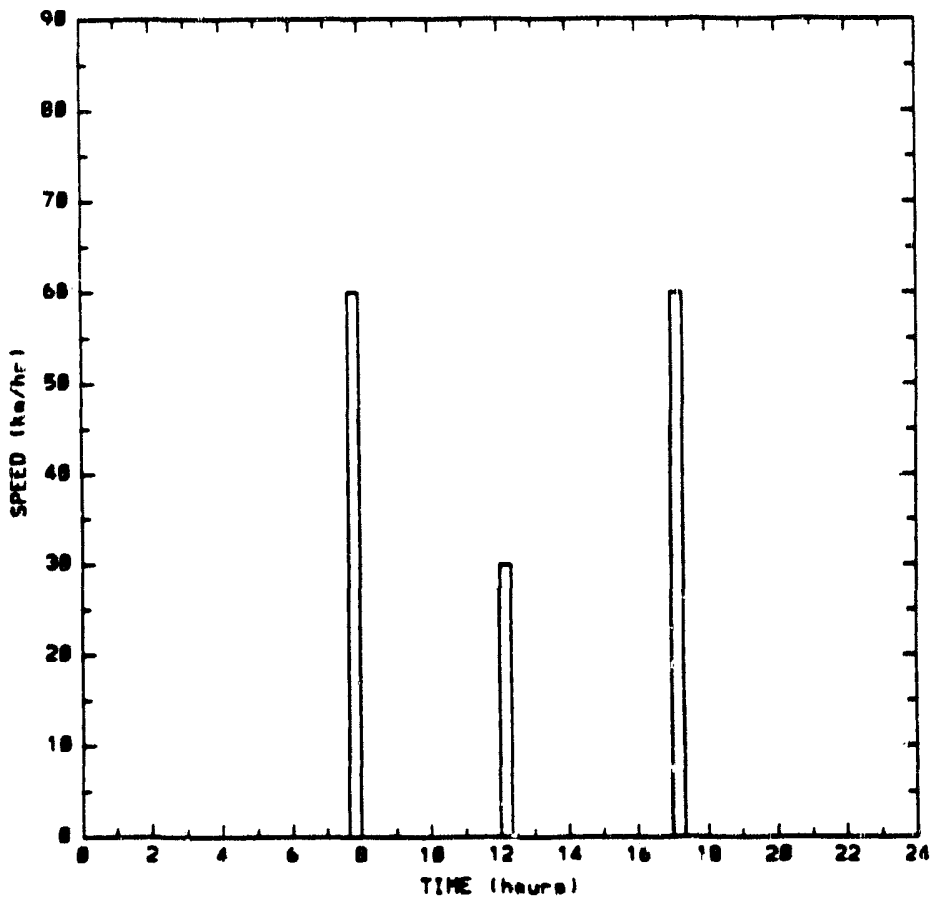

Figure 9-2. Plot of Vehicle Dse Pattern

No. 2 (Time of Day Starts at Midnight) 


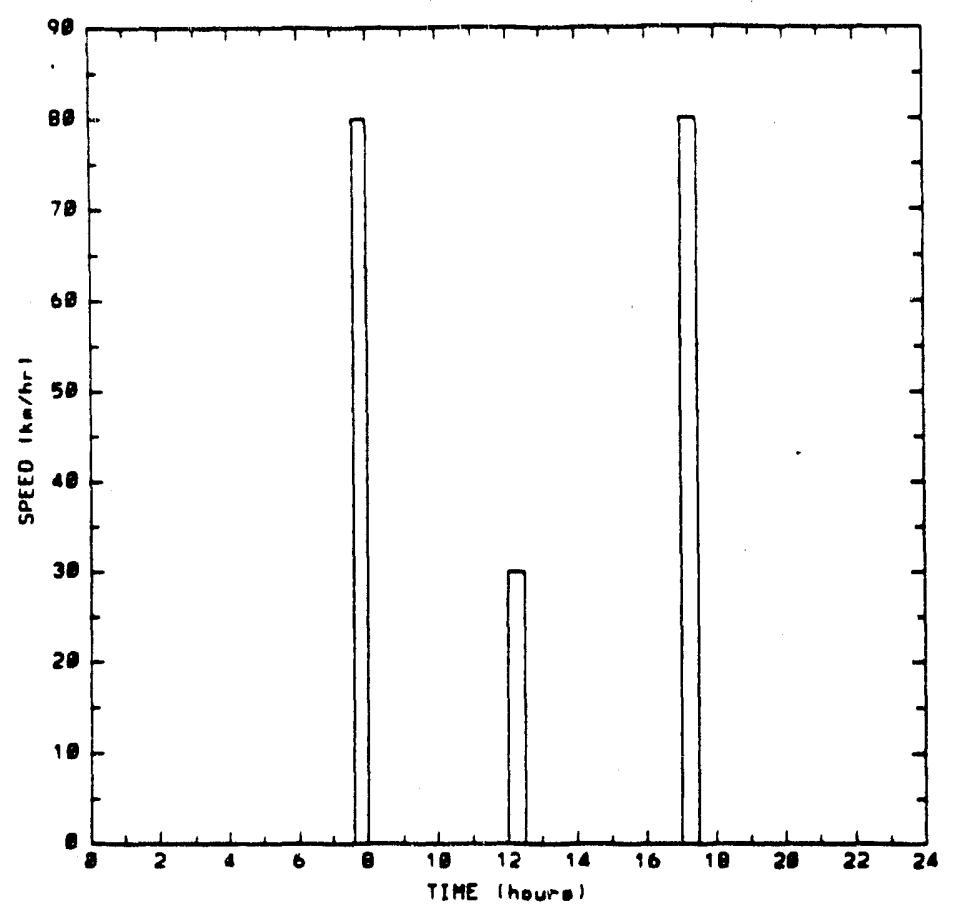

Figure 9-3. Plot of Vehicle Use Pattern No. 3 (Time of Day Starts at Midnight)

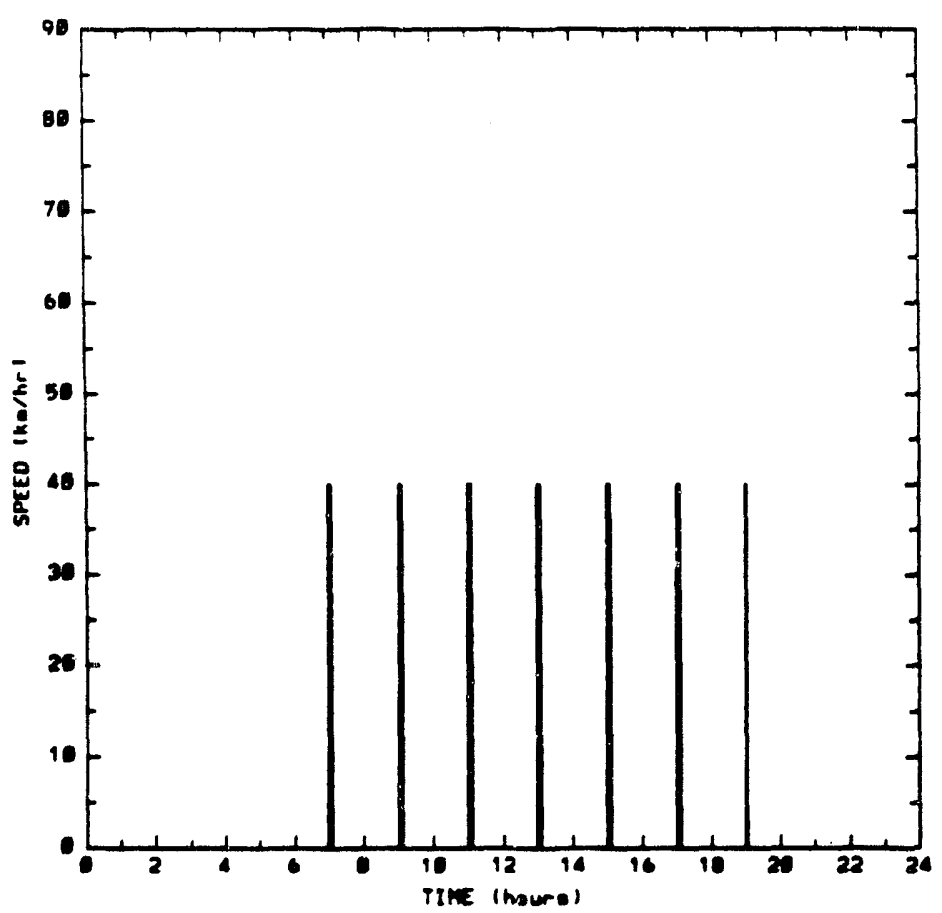

Figure 9-4. Plot of Vehicle Use Pattern No. 4 (Time of Day Starts at Midnight) 


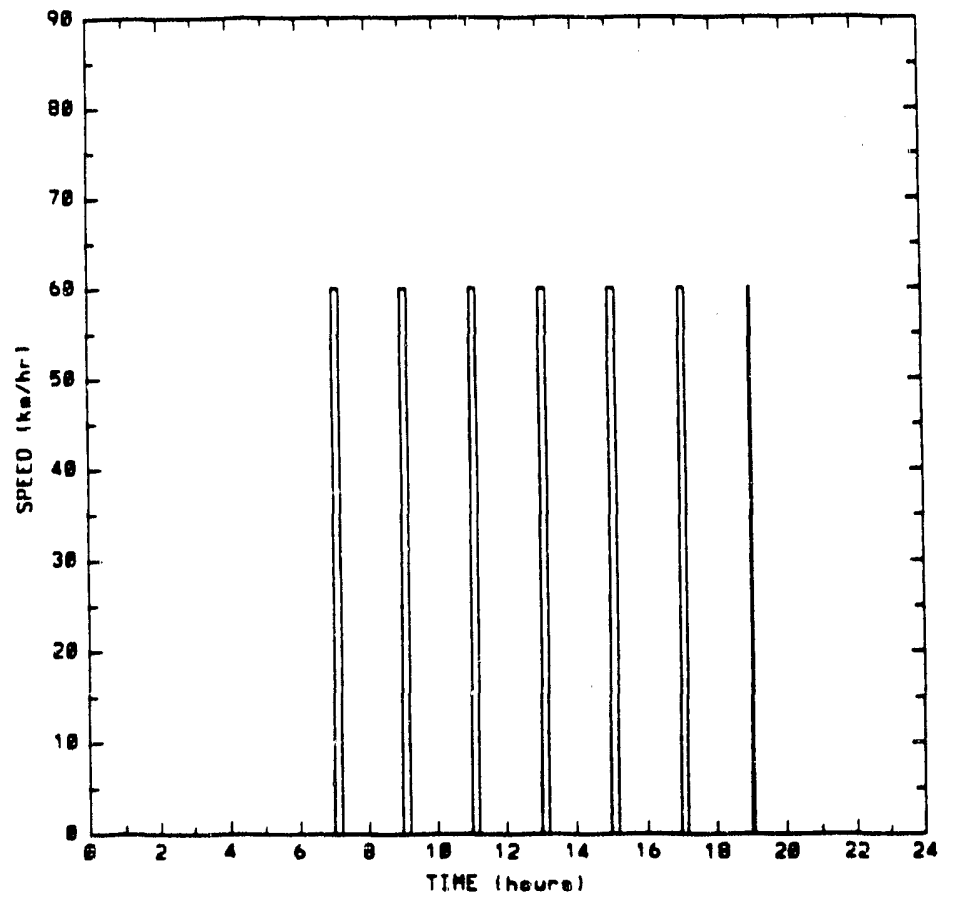

Figure 9-5. Plot of Vehicle Use Pattern No. 5 (Time of Day Starts at Midnight) 
The purpose of the battery model is to provide estimates for life and energy efficiency for different types of batteries likely to be used in electric and solar electric vehicles. The life modeling in turn enables estimation of economic depreciation. Three battery types were used in the simulation corresponding to:

1. High Ferformance Sealed Lead Acid.

2. Low Performance Industrial Type Lead Acid.

3. Traction Type Sodium Sulphur.

\subsection{LIFE MODELING}

The existence of a "wearout" function, $F$, is postulated. For a new (healthy) battery, $F=0$, and at end of life, $F=1$. It is assumed that three mechanisms contribute to the wearout function, namely:

1. Calendar Effects

(These are effects such as grid corrosion and active material morphology change which are assumed proportionate to the passage of time.)

2. End of Charge Effects

(These are effects such as plate shedding, water loss and grid corrosion which are proportionate to the number of recharges; most of the "damage" takes place during the final portion of recharge when gas evolution takes place.)

3. Cycle Depth Effects

(These are effects such as plate shedding, fatigue corrosion, morphology change and grid-to-active anterial interface degradation. Damage per cycle is a nonlinear function of cycle depth.)

In that each of the three mechanisms just mentioned tends to weaken common elements, such as grid integrity or the grid to active material interface, it may be assumed that the total damage during a given time interval is proportionate to the sum of the component damages. Thus, on a per day basis,

$$
\Delta F=\Delta F_{\text {cal }}+\Delta F_{\text {charge }}+\Delta F_{\text {cycle }}
$$


where $\Delta F_{c a l}$ is the fractional damage due to calendar effects, $\Delta F_{\text {charge }}$ is the fractional damage due to recharge and $\Delta F_{\text {cycle }}$ is the fractional damage due to cycle depth effects.

Each of the three subcomponents may be expressed in terms of more conventional variables:

$$
\begin{aligned}
& \Delta F_{\text {cal }}=\frac{1}{T_{\text {cal }}}, \\
& \Delta F_{\text {charge }}=\frac{1}{N_{\text {charge }}}, \\
& \Delta F_{\text {cocle }}=\frac{1}{N_{c}} \cdot \frac{\left(e^{\alpha \times}-1\right)}{\left(e^{\left.\alpha x_{0}-1\right)},\right.}
\end{aligned}
$$

where $T_{\text {cal }}$ is the battery life under hypothetical conditions where no stresses due to charging or cyciing are present, $N_{\text {cherge }}$ is the recharge cycles to end of life (one per day) where colendar and cycle depth effects are excluded, $N_{c y}$ is the cycle life at depth of discharge equal $x_{0}$ where calendar and recharge effects re excluded; $\alpha$ is a sensitivity parameter which determines how cycle life varies with depth of discharge. A high value of a means that cycle life falls rapidly as depth of discharge is increased.

Paramefiers of Equations 10-2 through 10-4 are not generally available. Instead, the usual data express in situ cycle life as a function of Lepth of discharge $(x)$. Often, life is simply expressed in years of service versus cycle depth. Service life is sometimes given for $x-0$. $x-0.3$ and $x=0.8$. Denoting $T_{0}, T_{30}$ and $T_{80}$ as the service life which corresponds respectively to 08,30 and 80 depth of discharge, equations may be derived which give $T_{c a l}, N_{c h a g e}, N_{c y}$ and $\alpha$ in terms of these variables.

Based on experimental input, the following approximation is assumed

$$
T_{\text {cal }}=2 T_{0}
$$

where both $T_{\text {cal }}$ and $T_{0}$ are in years.

Under zero depth of discharge conditions,

$$
T_{a}-\frac{1}{T_{\text {cal }}}+\frac{365}{N_{\text {chere }}}
$$

From Equations $(10-5)$ and $(10-6)$ it follows that

$$
N_{\text {chere }}=720 T_{0}
$$


It can also be shown that

$$
\mathrm{N}_{c y}=\frac{365 \mathrm{~T}_{0} \mathrm{~T}_{80}}{\mathrm{~T}_{0}-\mathrm{T}_{80}}
$$

The value of a (as used in Equation 10-4) must be found by an iterative approach

$$
\begin{aligned}
& \alpha=1.25 \ln Z \\
& Z=Q /\left(Z^{0.375}-1\right)+1 \\
& Q=\frac{\left(T_{0}-T_{30}\right) T_{80}}{\left(T_{0}-T_{80}\right) T_{30}}
\end{aligned}
$$

Equations (10-1) through (10-4) were used in the simulation for the three battery types. Constants for the three battery types are listed in Table 10-1. Values of $T_{0}, T_{30}$ and $T_{80}$ were obtained from References 3 and 4. Values of $T_{\text {cal }}, N_{\text {charge }}, N_{c y}$ and $\alpha$ were in turn obtained from Equations 10-5 through $10-10$.

By reciprocating Equation 10-1, battery life (in days) is obtained. Thus, Iffe (in years) is equal to $1 /(365 \Delta F)$. Using this definition for life and the constants in Table 10-1, the life versus DOD plot of Figure 10-1 is obtained.

10.2

COST AND EFFICIENCY AND ENERGY STORAGE MODELLING

Battery cost is assumed proportional to the energy storage and battery mass. Cost per $\mathrm{kWh}$ (C5) and specific energy (E2) (both with respect to 100 depth of discharge at the 2 hour rate) are 1isted in Table 10-2 using data from References 3 and 4.

Table 10-1. Battery Life Constants

\begin{tabular}{|l|c|c|c|c|c|c|c|}
\hline \multicolumn{1}{|c|}{ Battery Type } & $\begin{array}{c}T_{0} \\
\text { (years) }\end{array}$ & $\begin{array}{c}T_{30} \\
\text { (years) }\end{array}$ & $\begin{array}{c}T_{80} \\
\text { (years) }\end{array}$ & $\begin{array}{c}T_{c a l} \\
\text { (years) }\end{array}$ & $N_{\text {charge }}$ & $N_{c y}$ & $\alpha$ \\
\hline $\begin{array}{l}\text { Type 1 - High } \\
\text { Performance } \\
\text { Sealed Lead } \\
\text { Acid }\end{array}$ & 5.0 & 3.0 & 0.5 & 10.0 & 3650 & 166 & 4.7 \\
\hline $\begin{array}{l}\text { Type 2 - } \\
\text { Industrial } \\
\text { Lead Acid }\end{array}$ & 10.0 & 6.0 & 3.0 & 20.0 & 7300 & 842 & 1.0 \\
\hline $\begin{array}{l}\text { Type 3 - } \\
\text { Sodium Sulphur }\end{array}$ & 12.0 & 8.5 & 4.0 & 24.0 & 8760 & 1095 & 2.0 \\
\hline
\end{tabular}




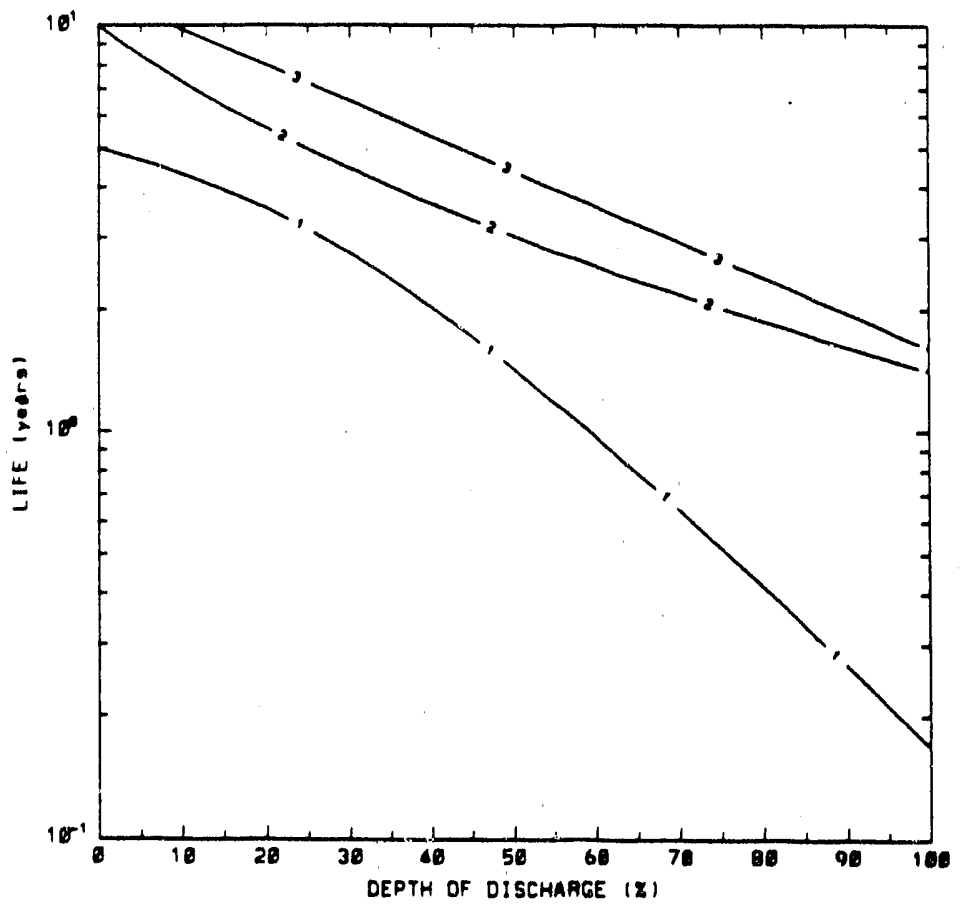

Figure 10-1. Battery Life Versus Depth of Discharge for Battery Types 1,2 and 3 Using Constants Listed in Table 10-1 
Because of energy losses associated with over-charge, a fixed energy loss is assumed with each cycle which is mass proportional and independent of cycle depth. Accordingly, the battery output energy $\left(E_{\text {out }}\right)$ is given by

$$
E_{\text {ous }}=\left(E_{\text {in }}-K_{B} M_{B}\right) \cdot N 3 \text {, }
$$

where $E_{i n}$ is the energy input and $N 3$ is the incremental efficiency, $K_{B}$ is a constant and $M_{B}$ is the battery mass.

Values of $C 5, E 2, K_{B}, N 3$ and $K_{B}$ are listed in Table 10-2.

Table 10-2. Miscellaneous Battery Constants

\begin{tabular}{|l|c|c|c|c|c|}
\hline Battery Type & $\begin{array}{c}C 5 \\
(\$ / \mathrm{kWh})\end{array}$ & $\begin{array}{c}E 2 \\
\left(\mathrm{Wh} / \mathrm{kg}^{\prime}\right)\end{array}$ & $\begin{array}{c}K_{B} \\
(\mathrm{~W} / \mathrm{kg})\end{array}$ & $\begin{array}{c}N 3 \\
(\mathbf{})\end{array}$ & $\begin{array}{c}M_{B} \\
(\mathrm{~kg})\end{array}$ \\
\hline Sealed Lead Acid & 75 & 37 & 0.677 & 90 & 443 \\
\hline Industrial Lead Acid & & 22 & 0.440 & 83 & 1136 \\
\hline Sodium Sulphur & 200 & 80 & 1.83 & 95 & 437 \\
\hline
\end{tabular}


Appendix A tabulates payback period and five other parameters for each of $3,240 \mathrm{PV}$ applications. The variables used in the computer simulation, listed in order of print-out nesting, include:

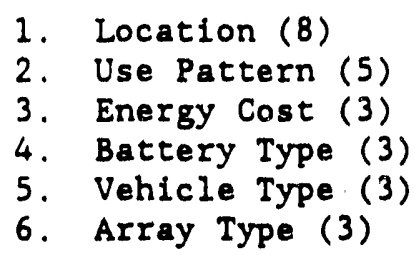

Each page of Appendix A corresponds to one location, one use pattern and one energy cost; each page contains data for all three battery types, all three vehicle types and all three array types. The outputs for each application are:

1. Up-Front Costs

(This is the sum of the array and converter plus installation costs. No provision is included for associated weight and aerodynamic penalties.)

2. Percent Energy Reduction

(This is the fractional decrease in recharge energy due to the addition of PV. No allowance is included for vehicle shadowing by trees and structures.)

3. Maximum Winter Depth of Discharge

(This is the maximum battery depth of discharge (DOD) where zero PV energy is assumed. Applications which exceed 60 to 80 are not considered viable for both economic and "range reliability" reasons.)

4. Percent DOD Reduction

(This is the yearly average reduction of battery DOD due to the inclusion of PV.)

5. Percent Battery Life Increase

(This is the fraction by which battery life is increased due to the inclusion of PV.)

\section{Payback Period}

(This is the time, in years, for the combined electricity and battery savings to equal the "up-front" cost of the array, converter and installation. No allowance is included for maintenance or the cost of money.) 
In order to estimate the payback period for a candidate application, select the simulated case whose parameters are closest to those of the candidate application.

Example 1 .

Determine tlie payback period for the case with the following parameters:

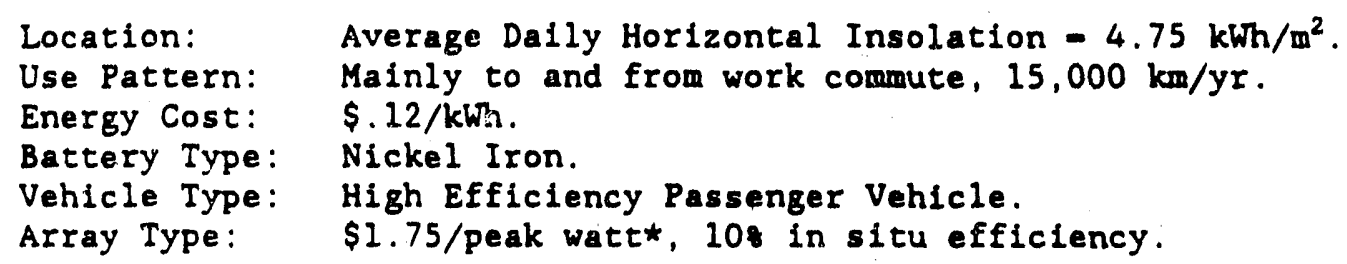

Selection of clesest simulation parameters:

The nearest location is Dallas-Fort Worth and the closest use pattern is Use Pattern Number 2 (see Table 9-1). The nearest energy cost is \$. $10 / \mathrm{kWh}$.

The nearest battery type is Type 3, the Sodium Sulphur. (see Tables 10.1 and 10.2). The reason for this selection is that both the Nickel Iron and Sodium Sulphur are fairly expensive in terms of initial cost per kWh. Also both batteries have lives which are relatively insensitive to cycle depth.

Vehicle Type 1 is chosen as this vehicle is an advanced, efficient comnuter type vehicle (see Table 8-1). Finally, Array Type 1 is chosen in that cost per watt and efficiency are in the same ballpark (see Table 6-1).

From the above, the closest simulation case is identified on page A51; the payback period is 21.3 years.

\section{2}

\section{SENSITIVITY ANALYSIS}

By noting the change in payback when one parameter is changed (and all others are held constant), it is possible to determine payback sensitivity with respect to the given parameter.

\footnotetext{
*Based on $1000 \mathrm{w} / \mathrm{m}^{2}$ insolation
} 
Example 2.

Determine the parametric sensitivities for Example 1:

1. Location Sensitivity.

Moving from Dallas-Forth Worth $\left(4.49 \mathrm{kWh} / \mathrm{m}^{2}\right)$ to Sacramento $\left(5.04 \mathrm{kWh} / \mathrm{m}^{2}\right)$, the payback period falls from 21.3 to 19.1 years. Accordingly, the sensitivity is

$$
\left(\frac{10.2-21.3}{10.2}\right) /\left(\frac{5.04-4.40}{5.04}\right)=-1.05 \text {. }
$$

Thus, a 18 increase in solar insolat ion reduces the payback period by about 18 .

2. Use Pattern Sensitivity.

Switching from Use Pattern 2 (18250 km/yr) to Use Pattern 1 $(9125 \mathrm{~km} / \mathrm{yr})$, the payback period increases from 21.3 to 23.9 years. Accordingly, the sensitivity is

$$
\left(\frac{23.0-21.3}{23.0}\right) /\left(\frac{0125-18250}{0125}\right)--0.109 \text {. }
$$

Hence, a 18 increase in yearly mileage reduces the payback period by about 0.18 .

3. Energy Cost Sensitivity.

Switching from $\$ 0.10 / \mathrm{kWh}$ to $\$ 0.20 / \mathrm{kWh}$ electricity cost, the payback period falls from 21.3 to 14.2 years. Accordingly, the sensitivity is

$$
\left(\frac{14.2-21.3}{16.2}\right) /\left(\frac{0.20-0.10}{0.20}\right)--1.00 \text {. }
$$

Thus, a increase in the cost of electricity reduces the payback period by about 1 .

4. Battery Type Sensitivity.

Switching from a high cost battery whose cycle life is insensitive to DOD (Sype 3 Battery) to a noderately expensive battery such as Type 2 which has a greater life sensitivity to DOD reduces the payback period from 21.3 years to 16.8 years. Note, however, that when the low cost, high DOD sonsitivity Type 1 battery is substituted, the payback period increases to 21.0 years. This behavior may be expected in that as battery cost falls, payback perlod will Increase, and that as DOD sensitivity increases, payback period will increase. 
5. Vehicle Type Sensitivity.

Switching from Vehicle Type 1 to Vehicle Type 2 (which is similar but less efficient), the payback period decreases slightly. (In terms of energy per mile, the sensitivity is about -0.12 ; $1 . e .$, a 1 increase in road-load causes the payback period to fall by about 0.18 .) The negative value is expected in that as road-load increases, DOD must also increase. As DOD increases, the payback period falls because of the enhanced benefits of battery life extension.

6. Array Type Sensitivity.

Changing from Array Type 1 (8) Efficiency, $\$ 150 / \mathrm{m}^{2}$ ) to Array Type 2 (25 Efficiency, $\$ 500 / \mathrm{m}^{2}$ ) causes the payback period to fall from 21.3 to 15.3 years. In that Array Types 1 and 2 have roughly the same cost per watt, most of the difference may be attributed to efficiency. The corresponding sensitivity is 0.23 ; i.e., a 108 improvement in efficlency (same cost per watt) will cause a 2.3 reduction in the payback period. 
Several observations are in order concerning payback.

1. Payback periods range from a low of 0.5 year (see $\mathrm{Pg}$ Al5) to a high of 166 years (see $\mathrm{Pg} \mathrm{A107).} \mathrm{Accordingly,} \mathrm{the} \mathrm{economic}$ viability of $\mathrm{PV}$ is dependent on application details. In some cases it may make sense, while in other cases, it may not.

2. The cases where payback is short include:

a. High solar insulation.

b. High vehicle use.

c. Use of low efficiency vehicle.

d. High cost energy.

e. High cost battery.

f. Use of battery with high life sensitivity to DOD.

g. Use of low cost array.

h. Use of high efficiency array.

3. Cases where payback is very short (e.g., less than 2 years) are due to excessive DOD. In other words, these are cases where a given electric vehicle is being driven to its range limits .. where battery life will be short and reliable range is exceeded.

4. Approximately 108 (or 300 cases) correspond to reasonable payback periods (e.g., less than 10 years) combined with reasonable vehicle use (DOD less than 608). Examples are (A9, Batt. 1, Veh. 1, Array 1, payback - 8.9 years), (A9, Batt. 2, Veh. 2, Array 2, payback - 9.5 years) and (A39, Batt. 1, Veh. 1. Array 1, payback - 10.0 years).

5. Short payback periods are due mainly to battery DOD sensitivity and not to high electricity costs. Accordingly, as batteries improve, payback periods will probably increase.

6. In the case of high cost energy ( . g., $\$ 0.20 / \mathrm{kWh})$, combined with low cost PV (Type 2) and high solar insolation, payback periods are typically less than 10 years .. Independent of battery and vehicle types provided use is at least moderate (greater than $18,000 \mathrm{~km} / \mathrm{yr}$ ).

7. Batteries which have high DOD sensitivity (0.8., Type 1) contribute to long payback times when DOD levels are aaintained low. 
While it is recognized that the present simulation is lacking in detail and input accuracy, it is none the less belleved useful in helping to identify applications where payback periods are acceptable. Accordingly, it is recommended that several such applications be identified and that an effort be extended to implement "PV Assist" .. and to track its economic effectiveness. Assuming that PV Assist gains a measure of acceptance, it would also be worthwhile to refine the present study so that it may become a key tool in the implementation of PV assisted EVs. While numerous recommendations for improvement will become apparent as future experience is gained, several recommendations are presently at hand.

1. Obtain a more extensive data base on battery life versus DOD for major battery types.

2. Obtain more extensive performance and economic data for candidate solar arrays.

3. Provide data and simulation of shading effects caused by trees and structures.

4. Provide data and simulation of PV degradation due to age and foreign matter.

5. Refine calculation of payback time to include effects of:
a. Interest rate.
b. Energy cost trends
c. PV maintenance costs.

6. Improve vehicle model so that vehicle cost reflects effects of added PV weight and vehicle up-grade required to support added PV weight. (While this effect is small, it is not negligible.)

7. Provide vehicle use patterns which are more detailed than liose presently used and correlate these use patterns with present gasoline vehicle use parterns.

8. Expand simulation so that the effects of array size are displayed. Rather than expand data $N$-fold, a better solution night be that certain "reference cases" (e.g., given battery or use pat:ern) be thusly expanded.

9. Expand data print-out to include:
a. Battery life (years) with and without PV.
b. Battery DOD with and without PV.
c. Battery depreciation rate with and without PV.
d. Electricity use with and without PV. 
10.

Restrict parametric combinations which are urlikely to be used -. so that print-out does not become excessively large. 


\section{References:}

1. "Estimates of Available Solar Radiation and Photovoltaic Energy Production for Various Tilted and Tracking Surfaces Throughout the U.S.", Sandia Report SAND85-2775, March 1986, D.F. Menicucci.

2. "Consumption Patterns of Household Vehicles," DOE Report DOË/EIA-0464(85), printed April 1987.

3. "Proceedings of the Fourth Annual Battery Conference on Applications and Advances", California State University, Long Beach, January 1989.

4. "Eighth International Electric Vehicle Symposium," Washington D.C., 1986.

Acknowledgment

The author expresses his appreciation to Mr. Steven Mikes of JPL for generation of the computer code used in this study. 
Appendix A

Printout of Six Parameters Associated With 3240 PV-Assist Simulations

Note: Data entries with $*$ correspond to cases where vehicle range (unassisted by PV) is exceeded. 


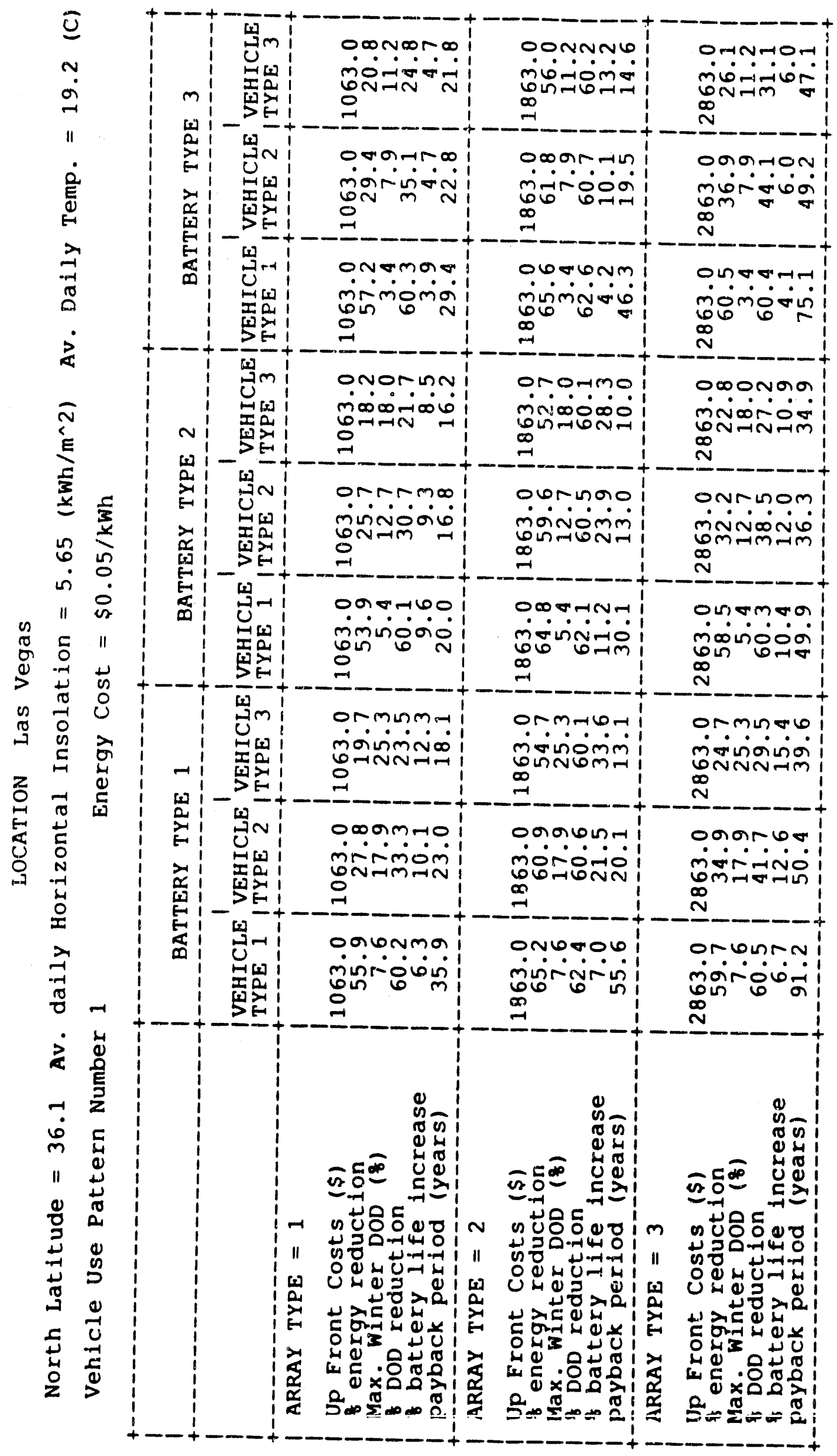




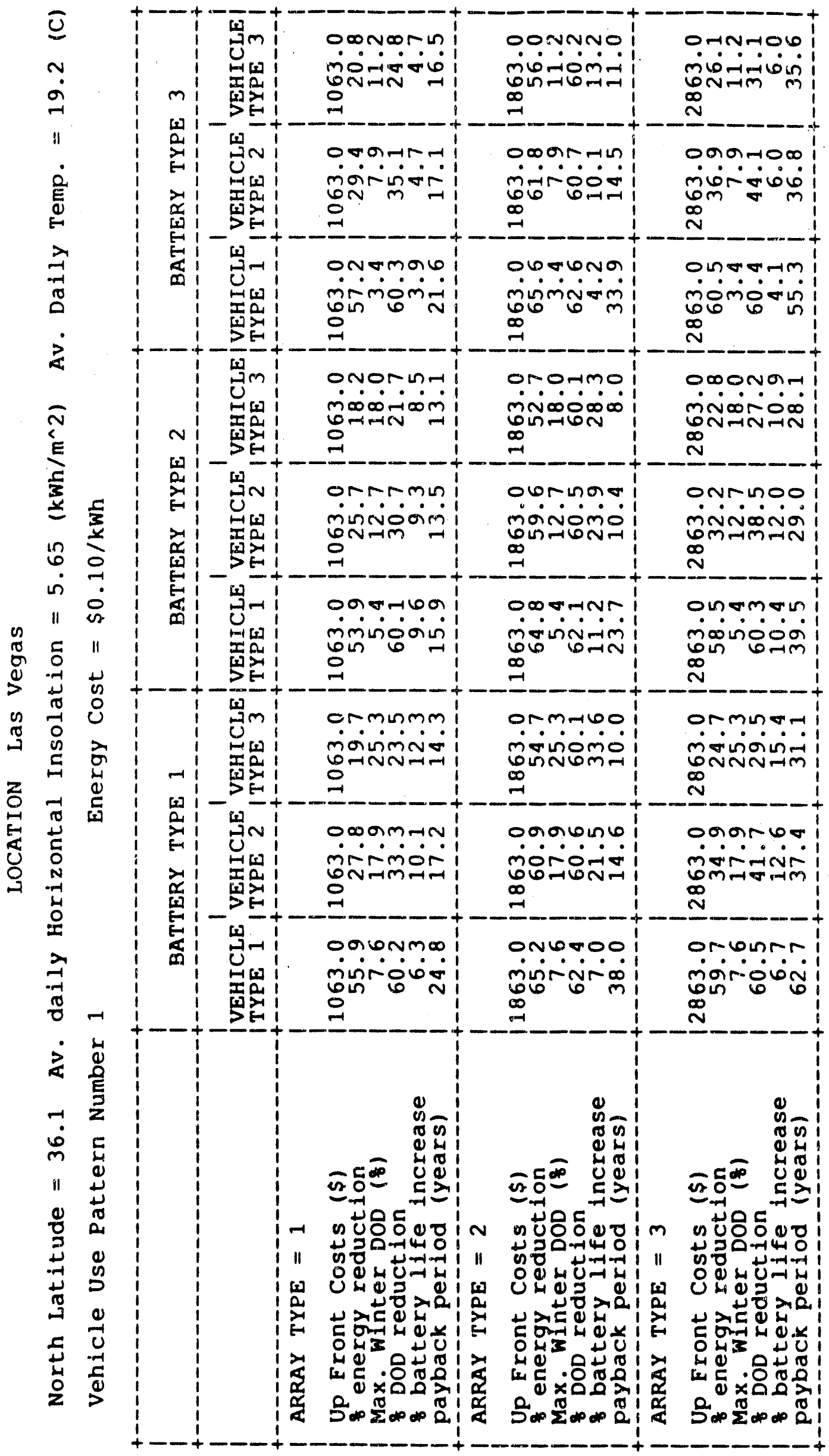




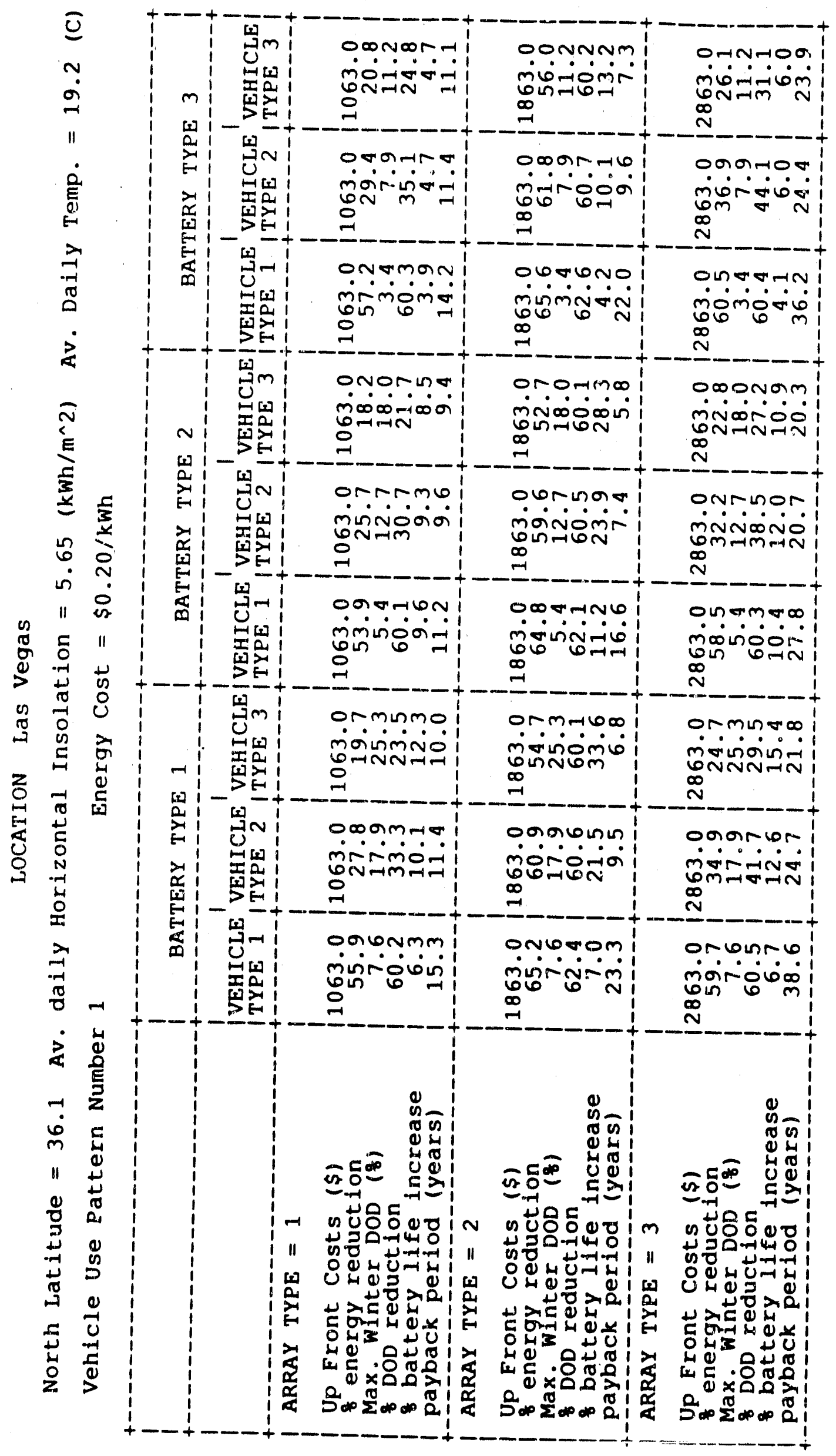




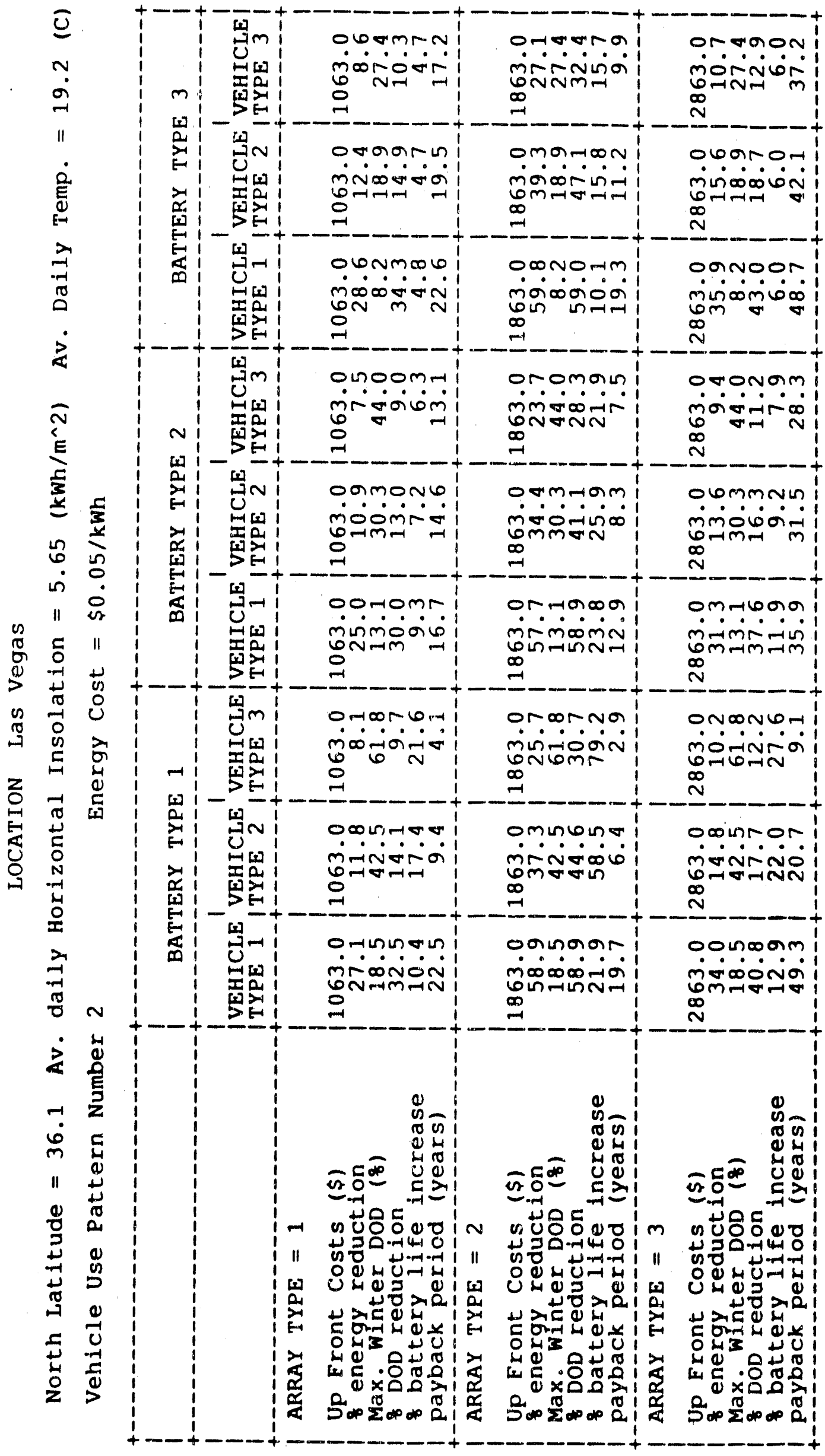




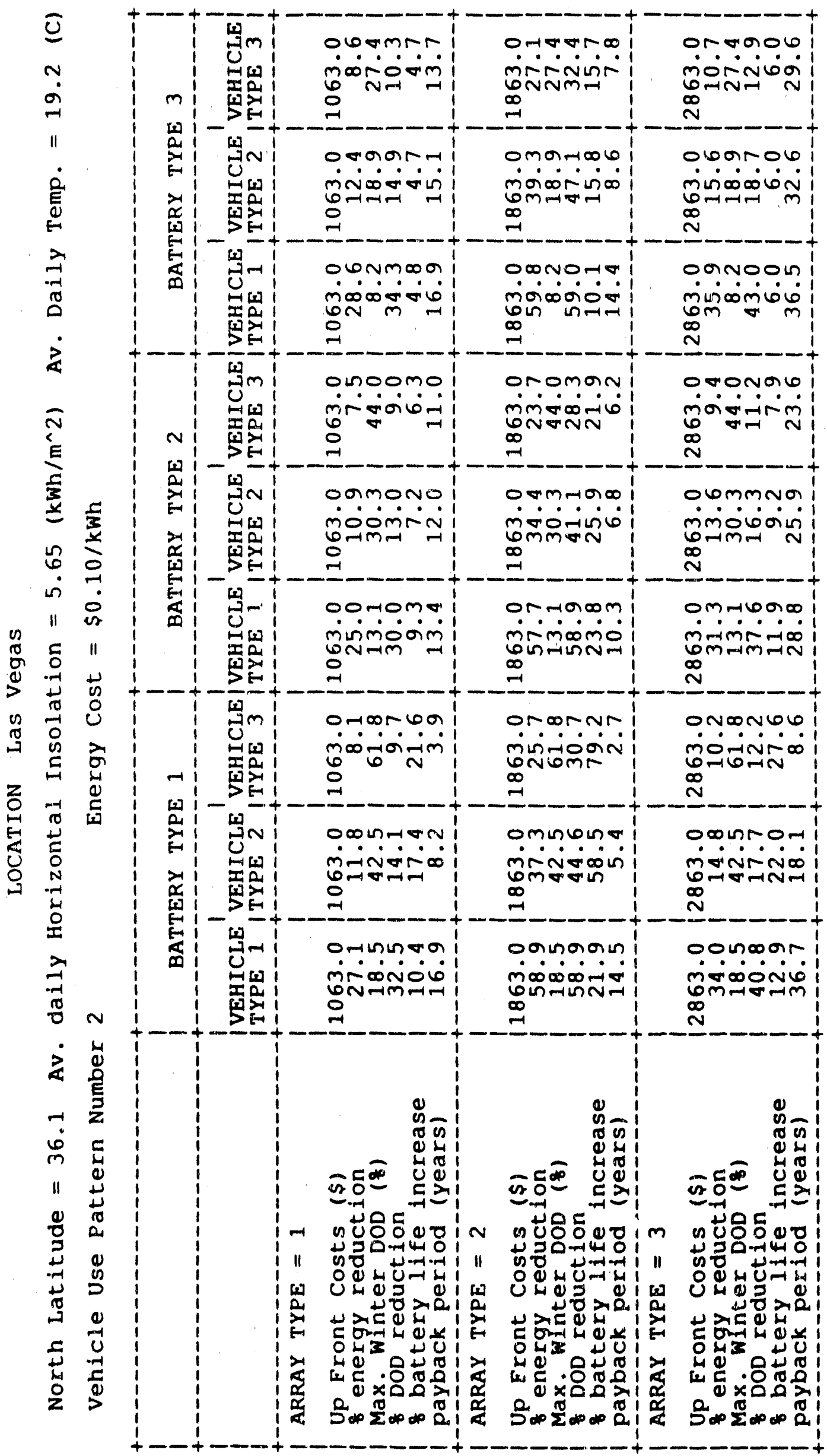




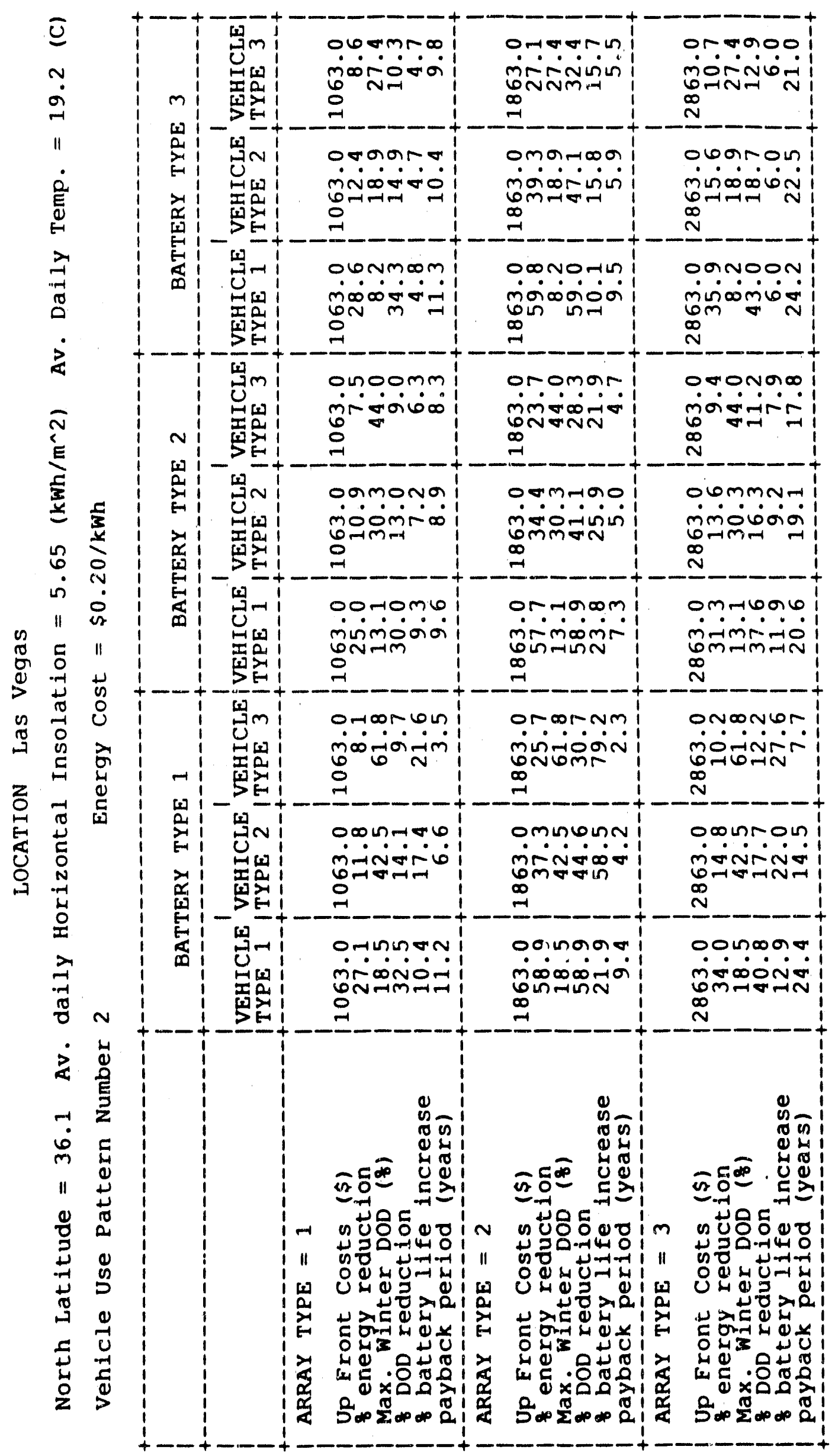




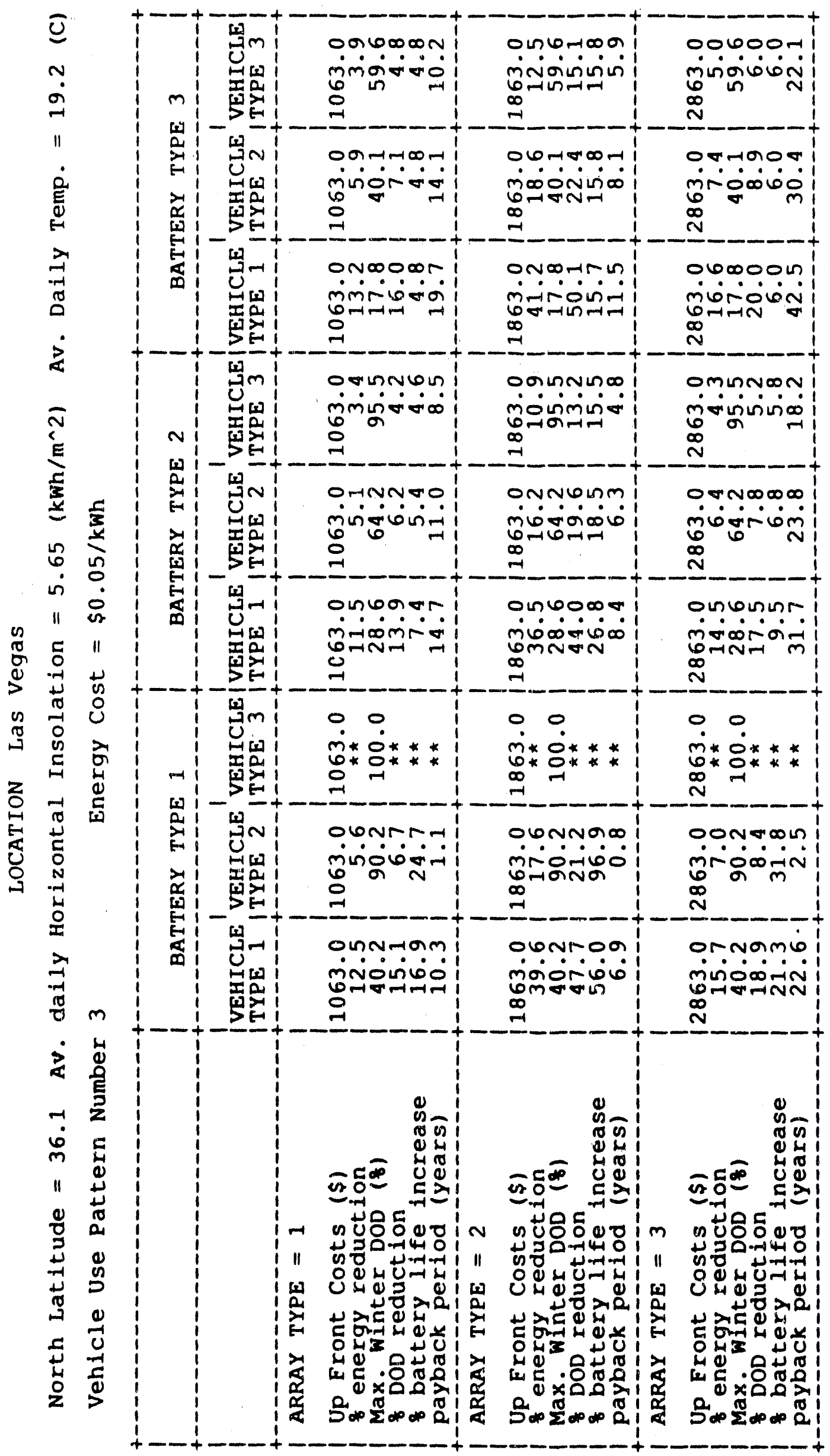




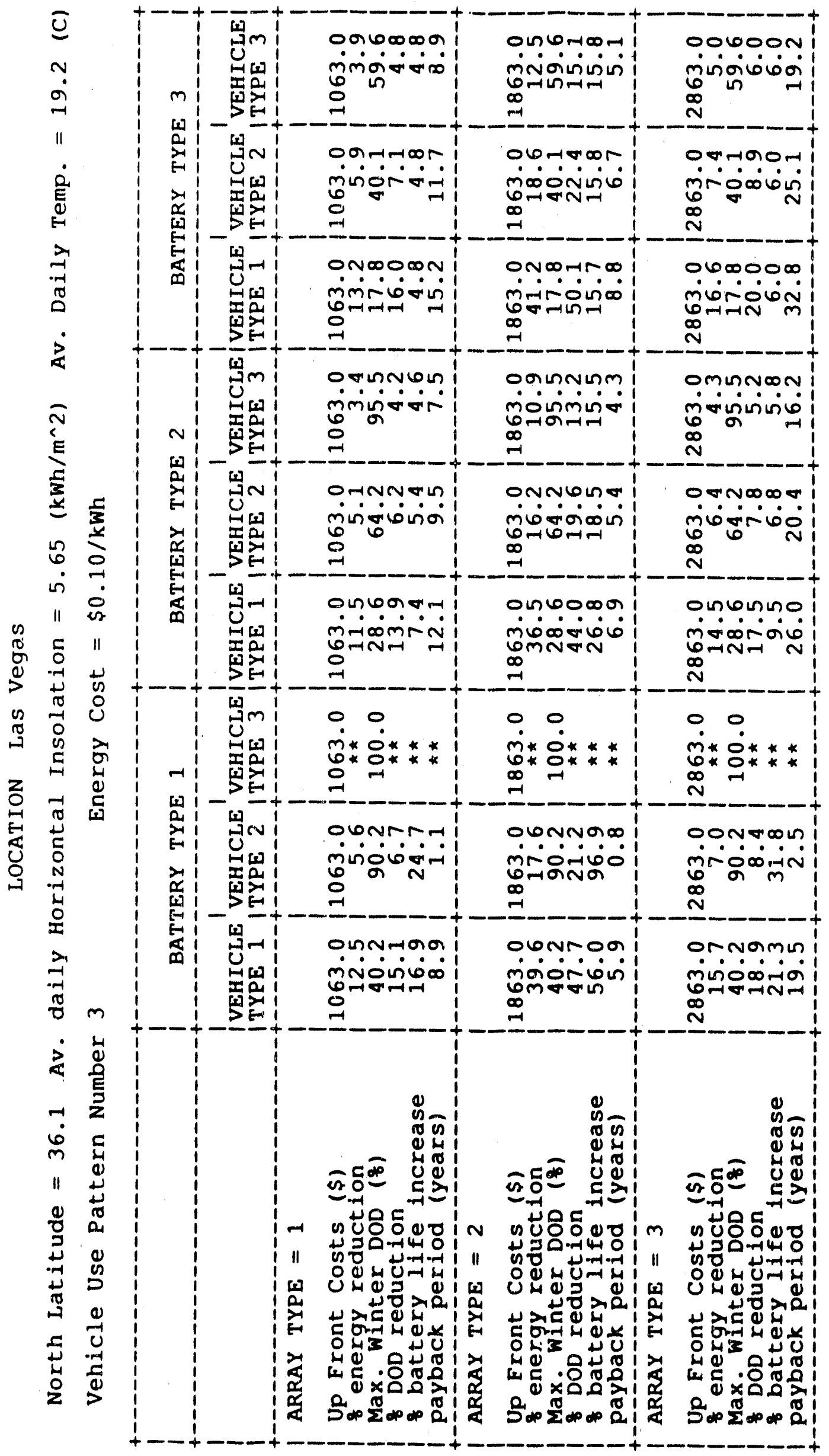




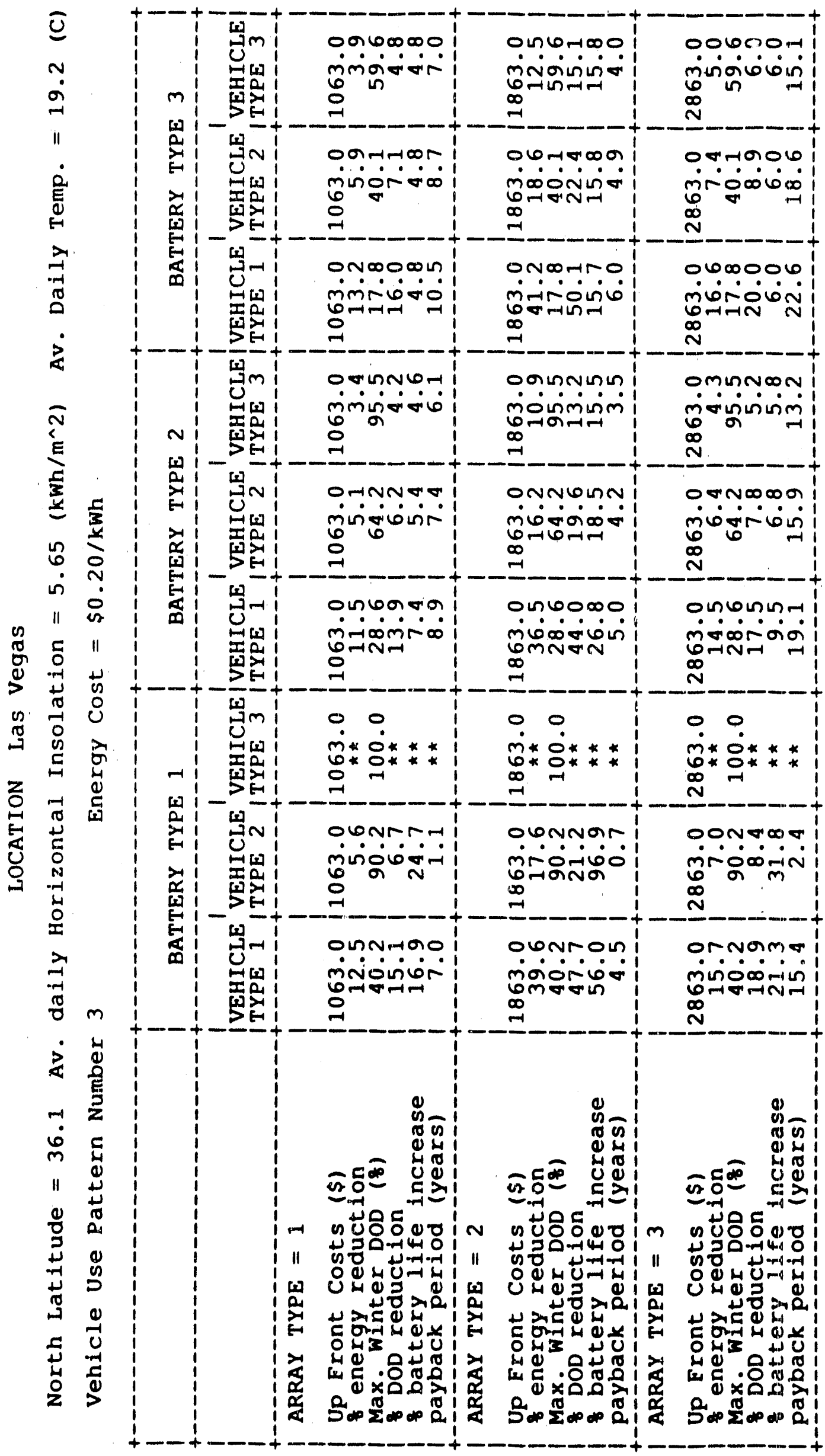




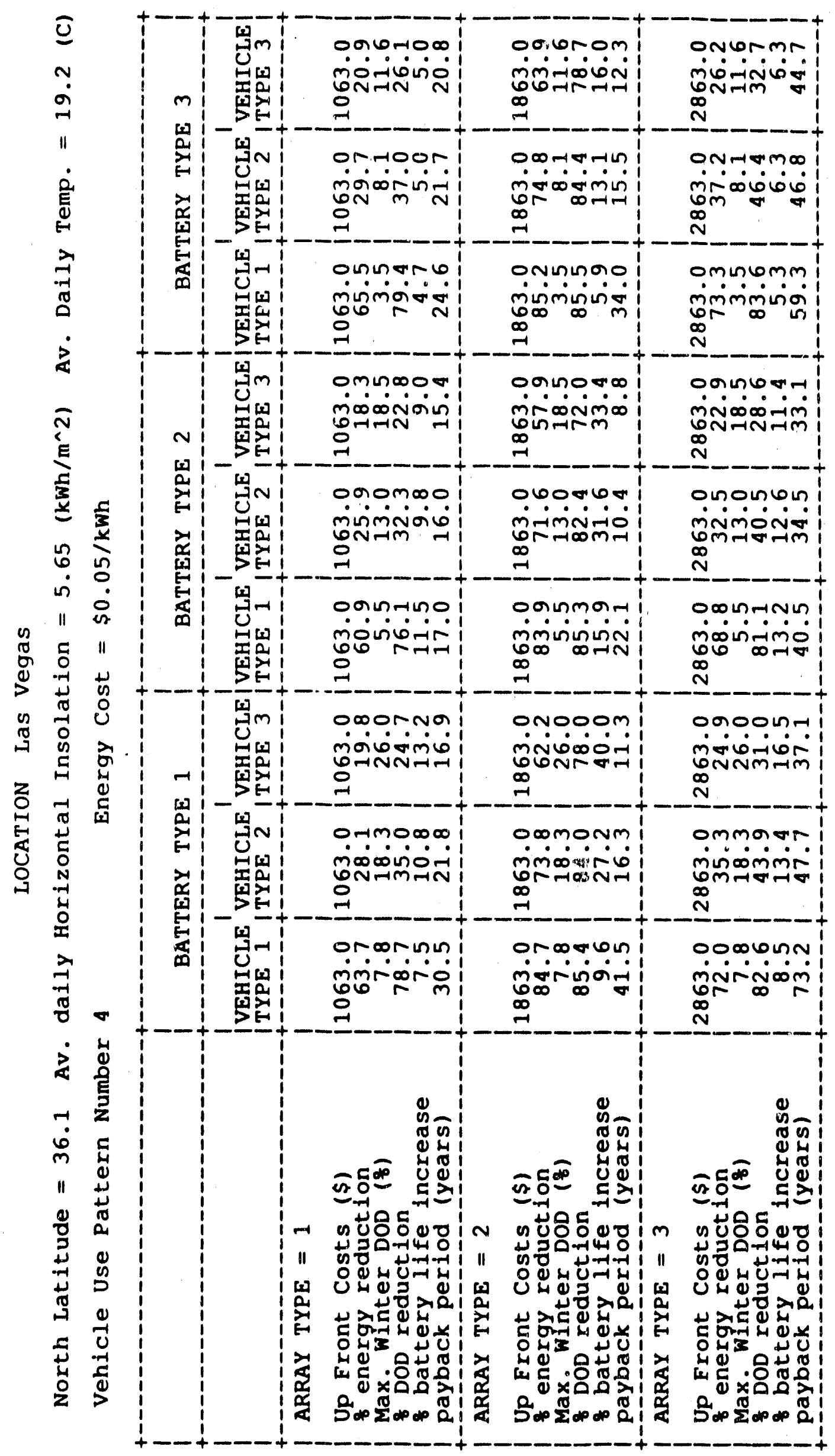




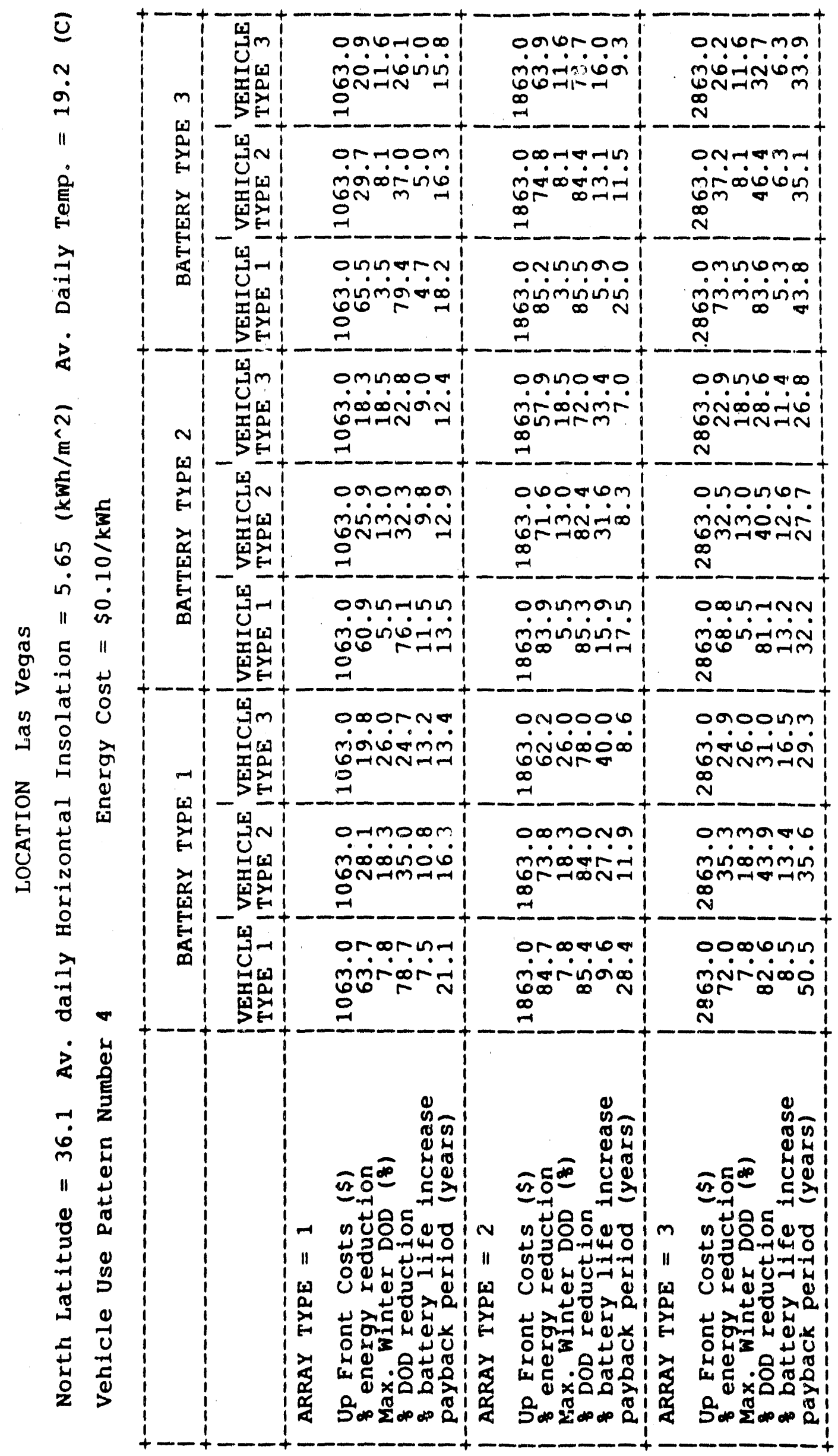




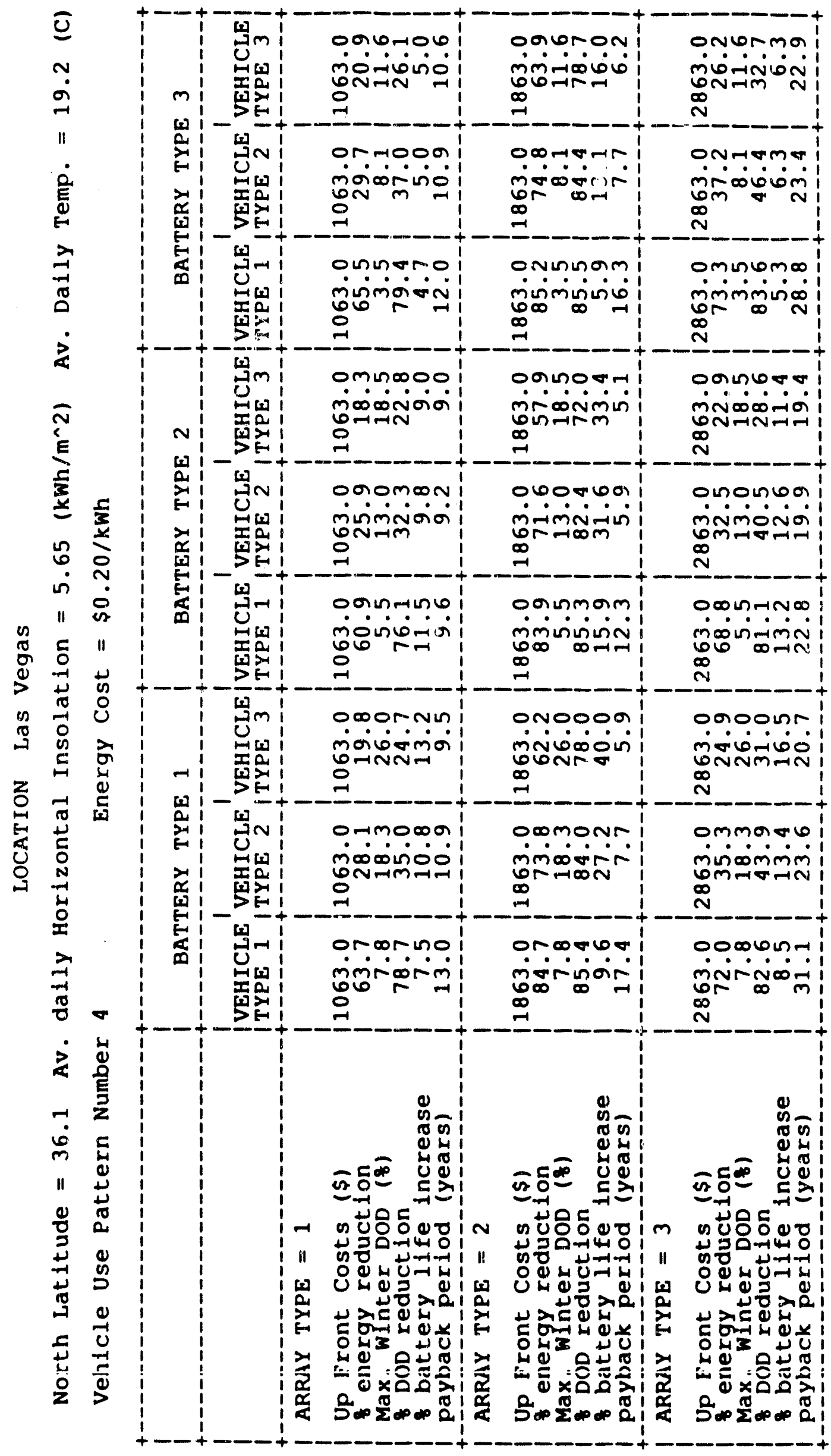




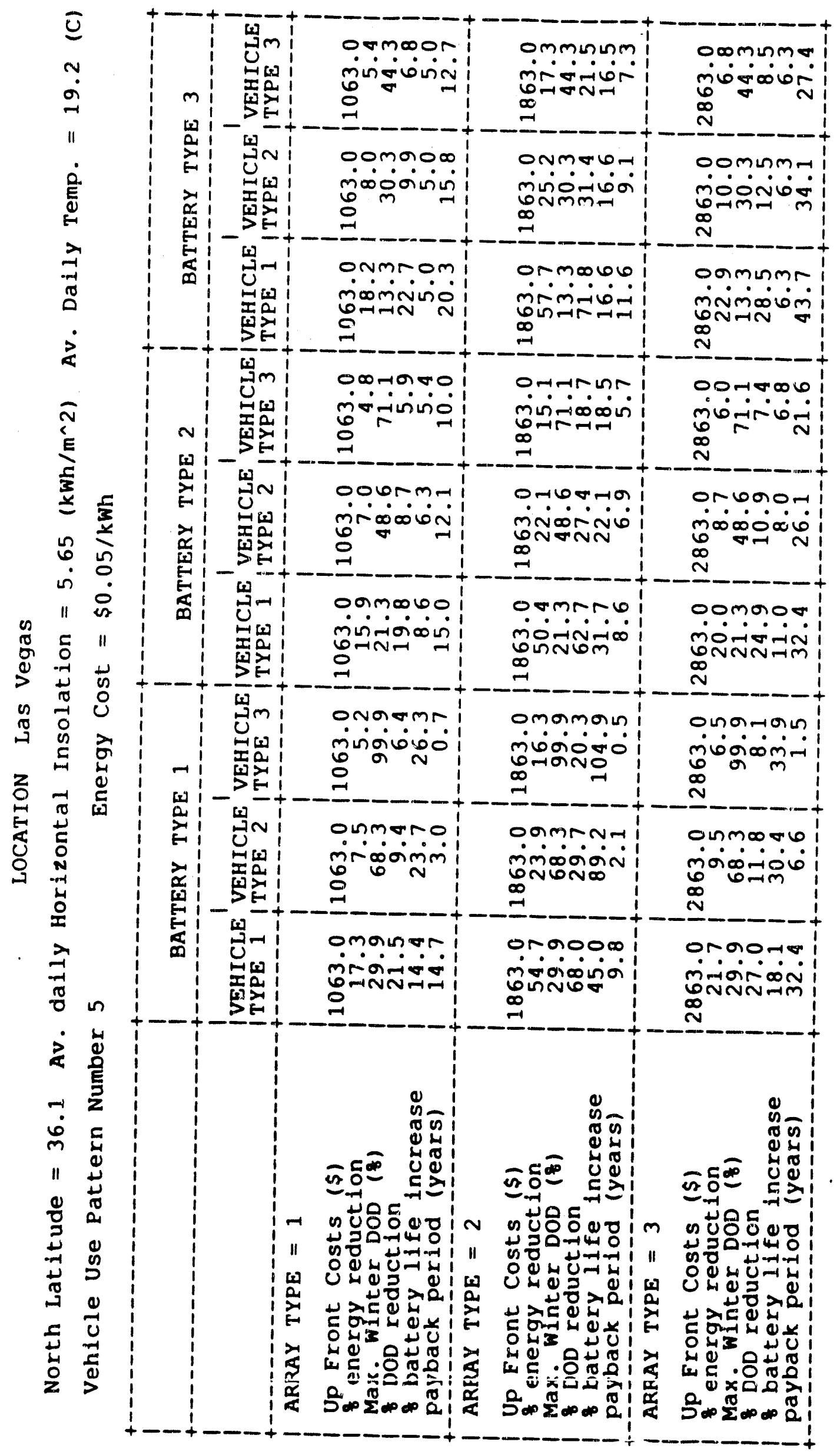




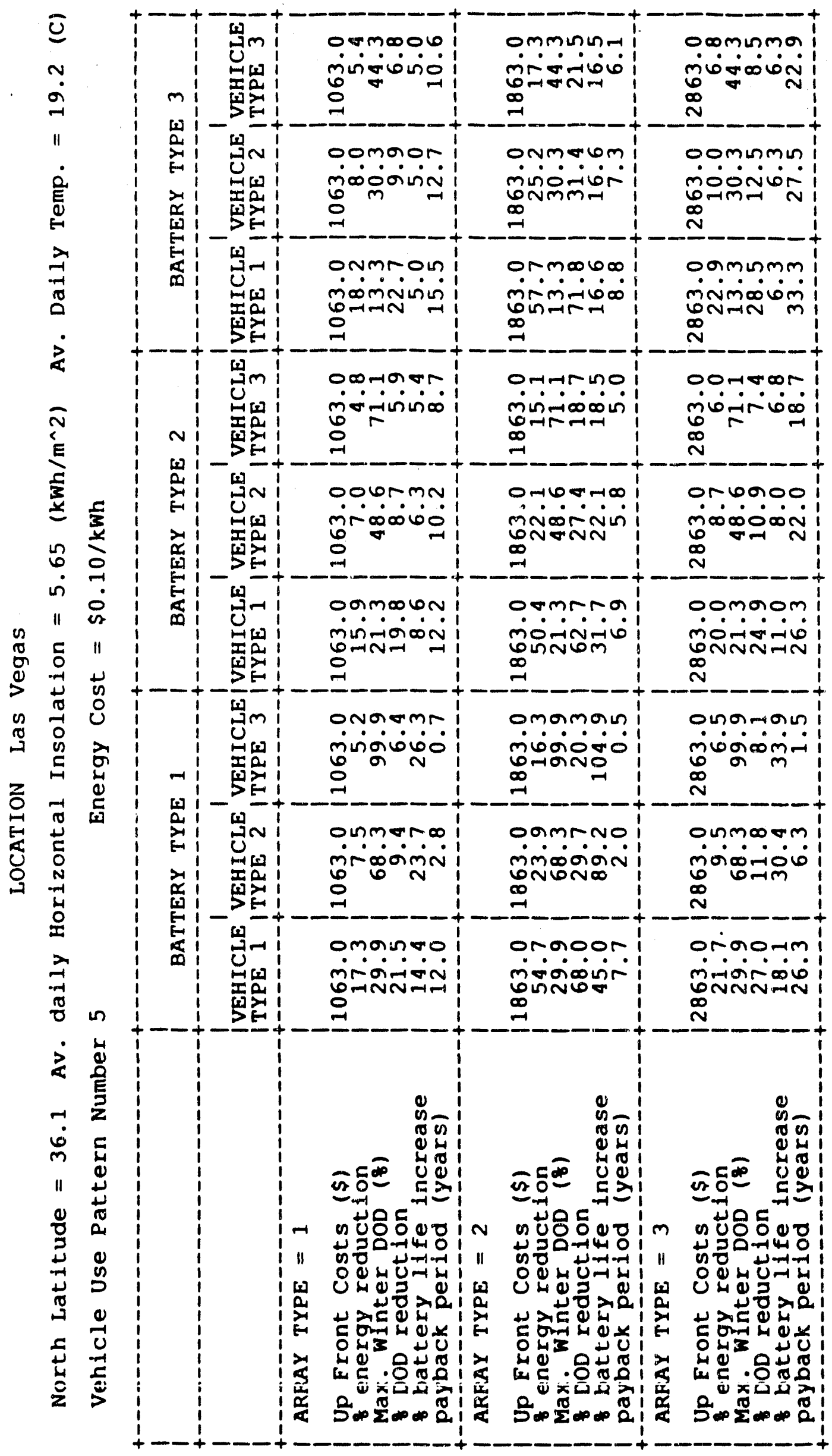




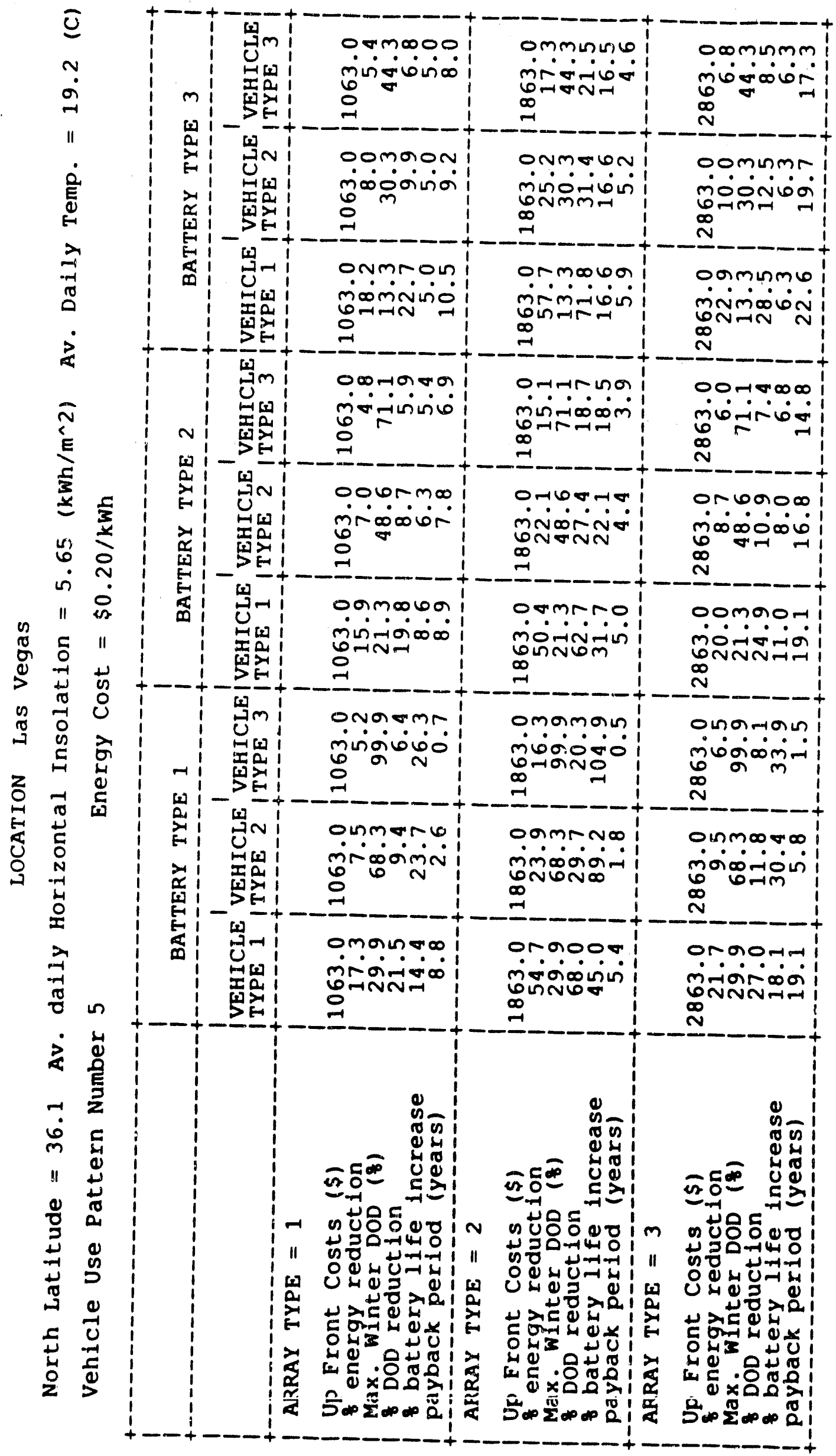




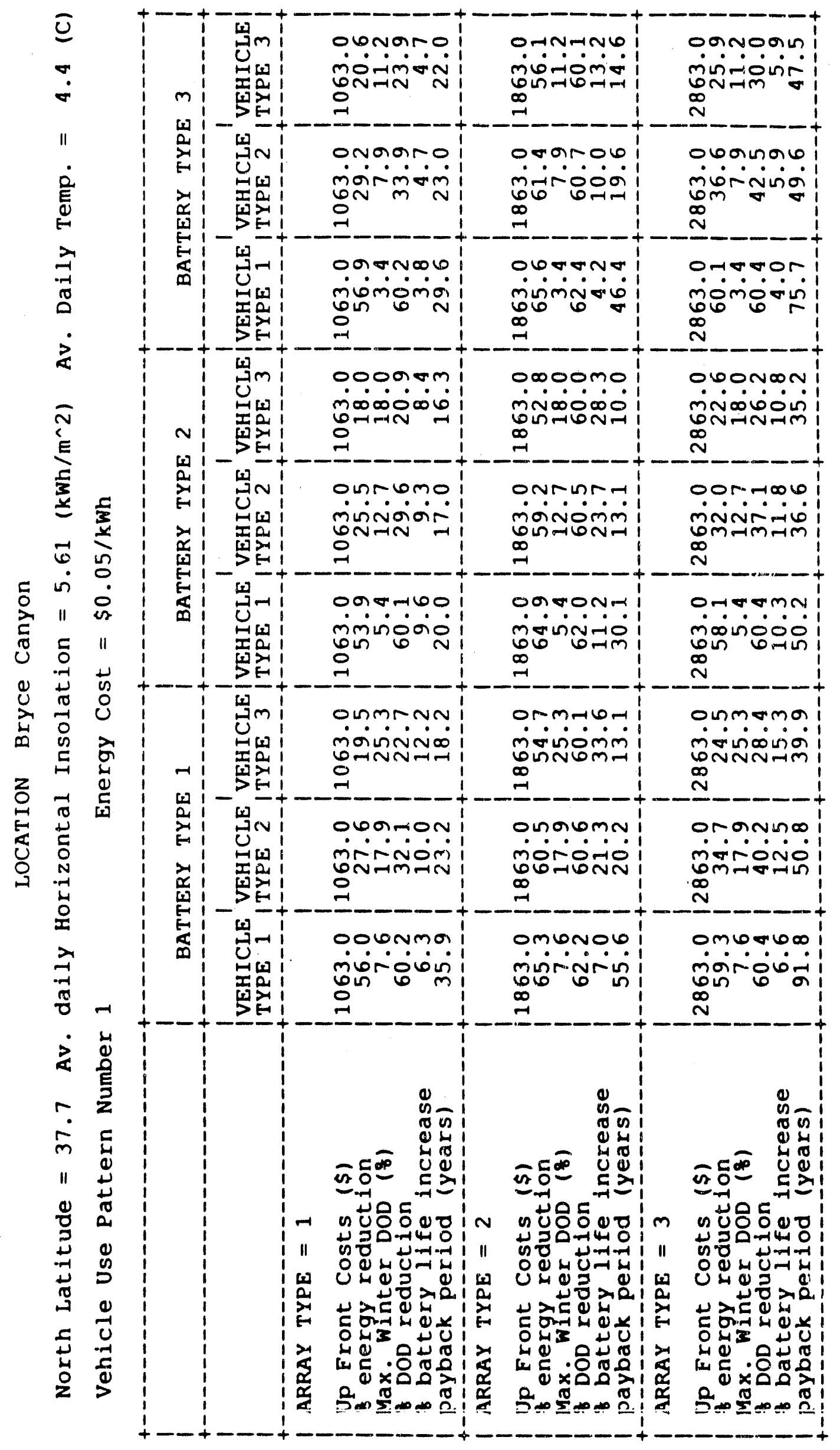




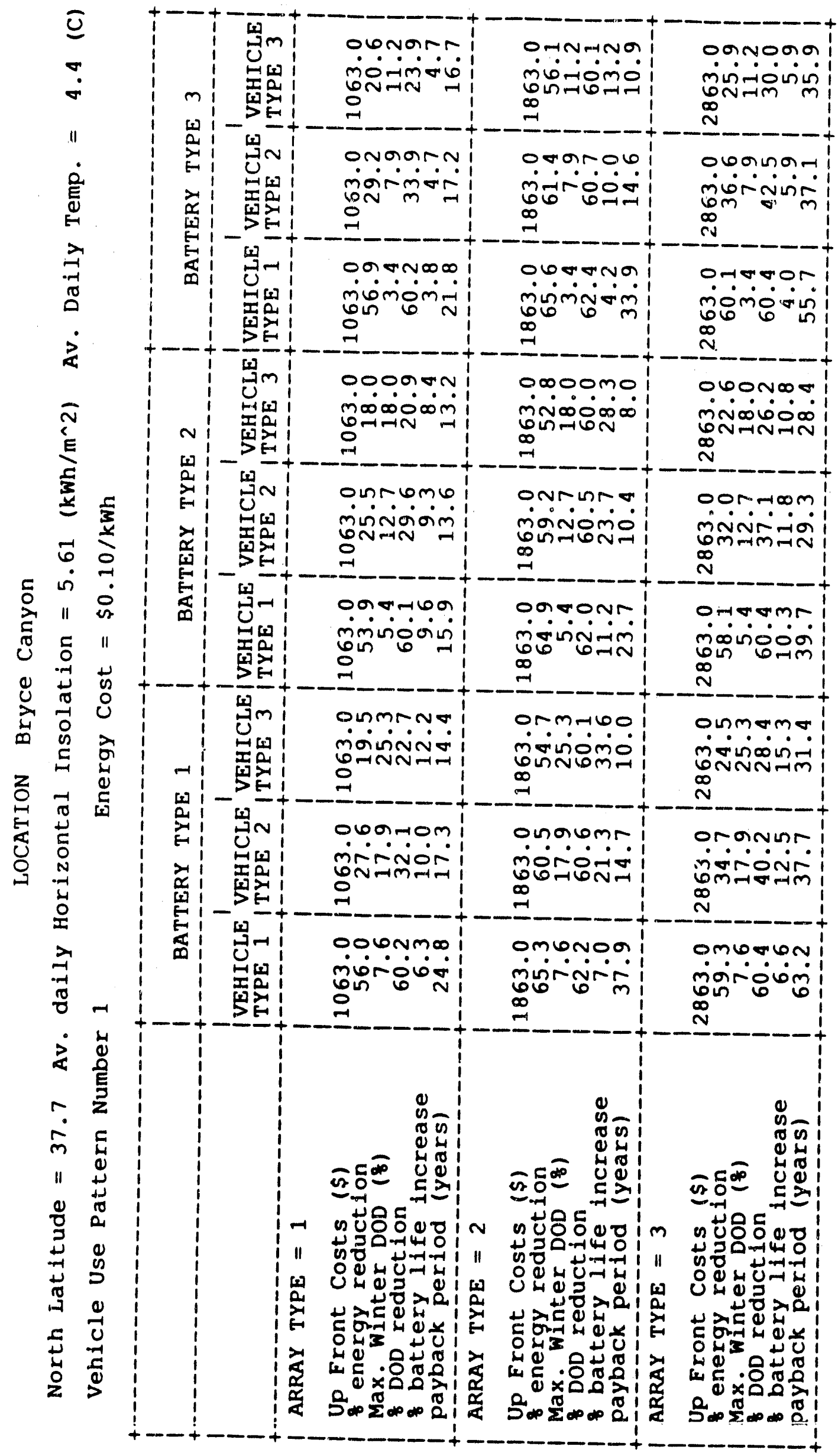




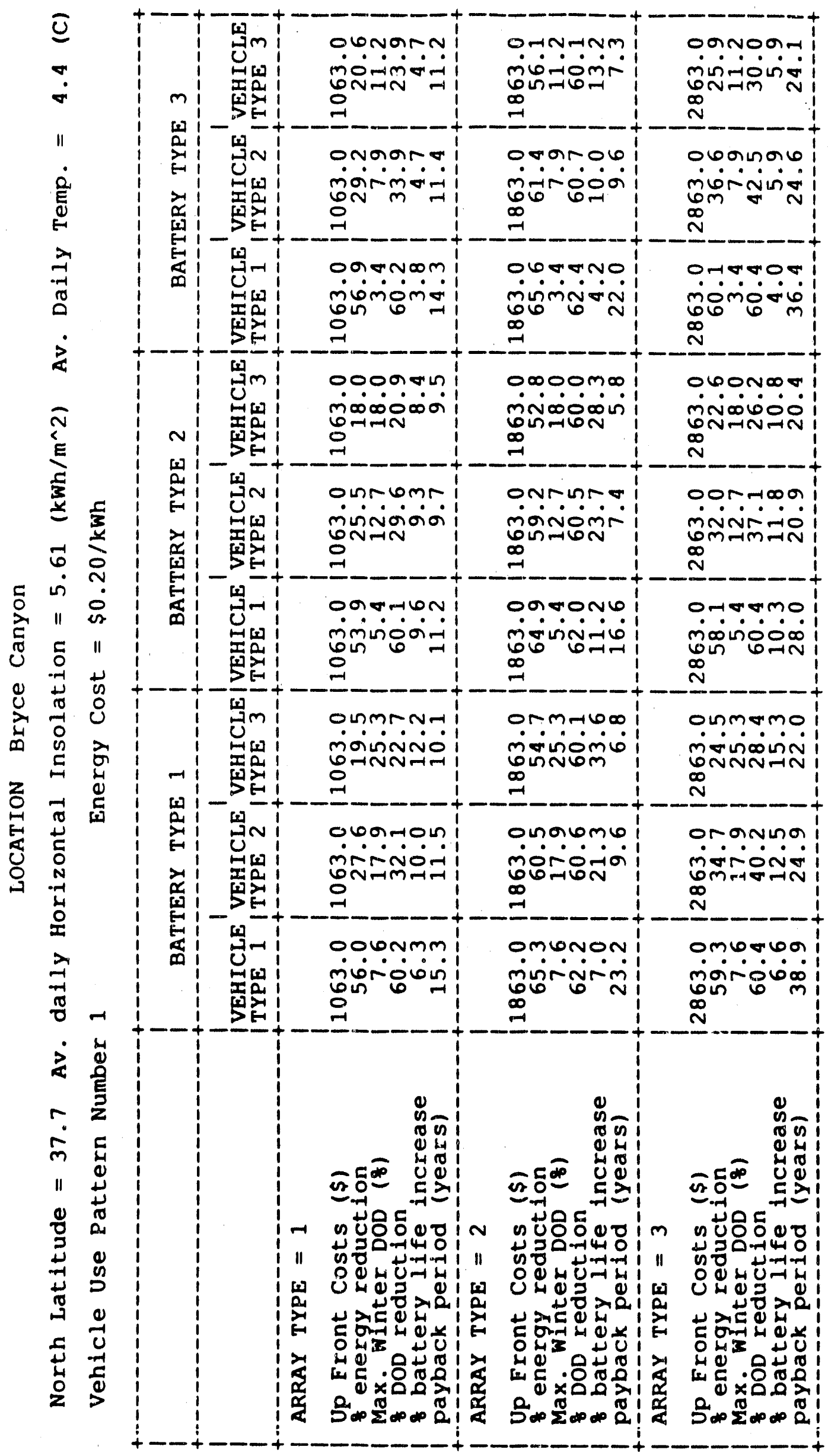




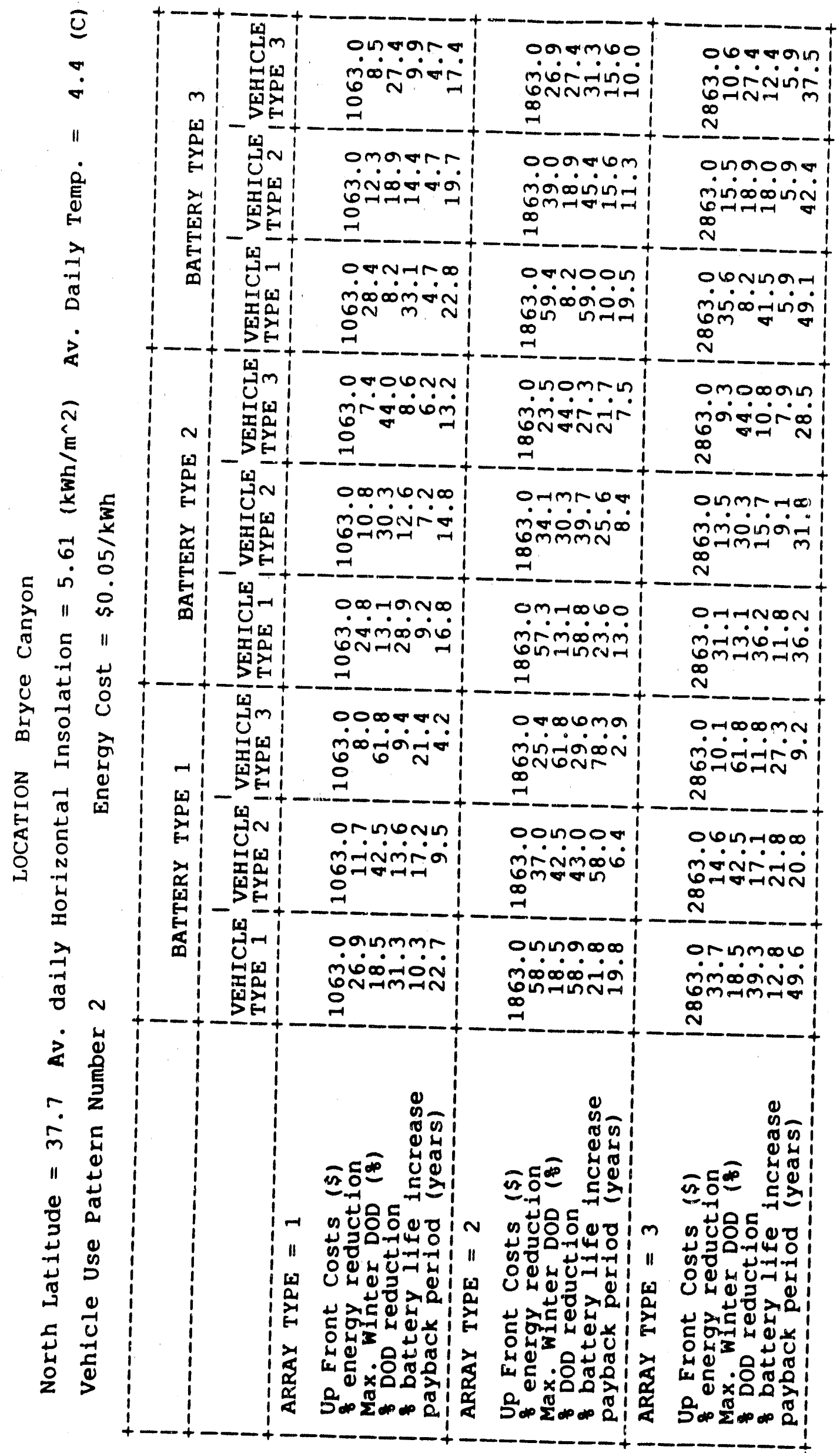




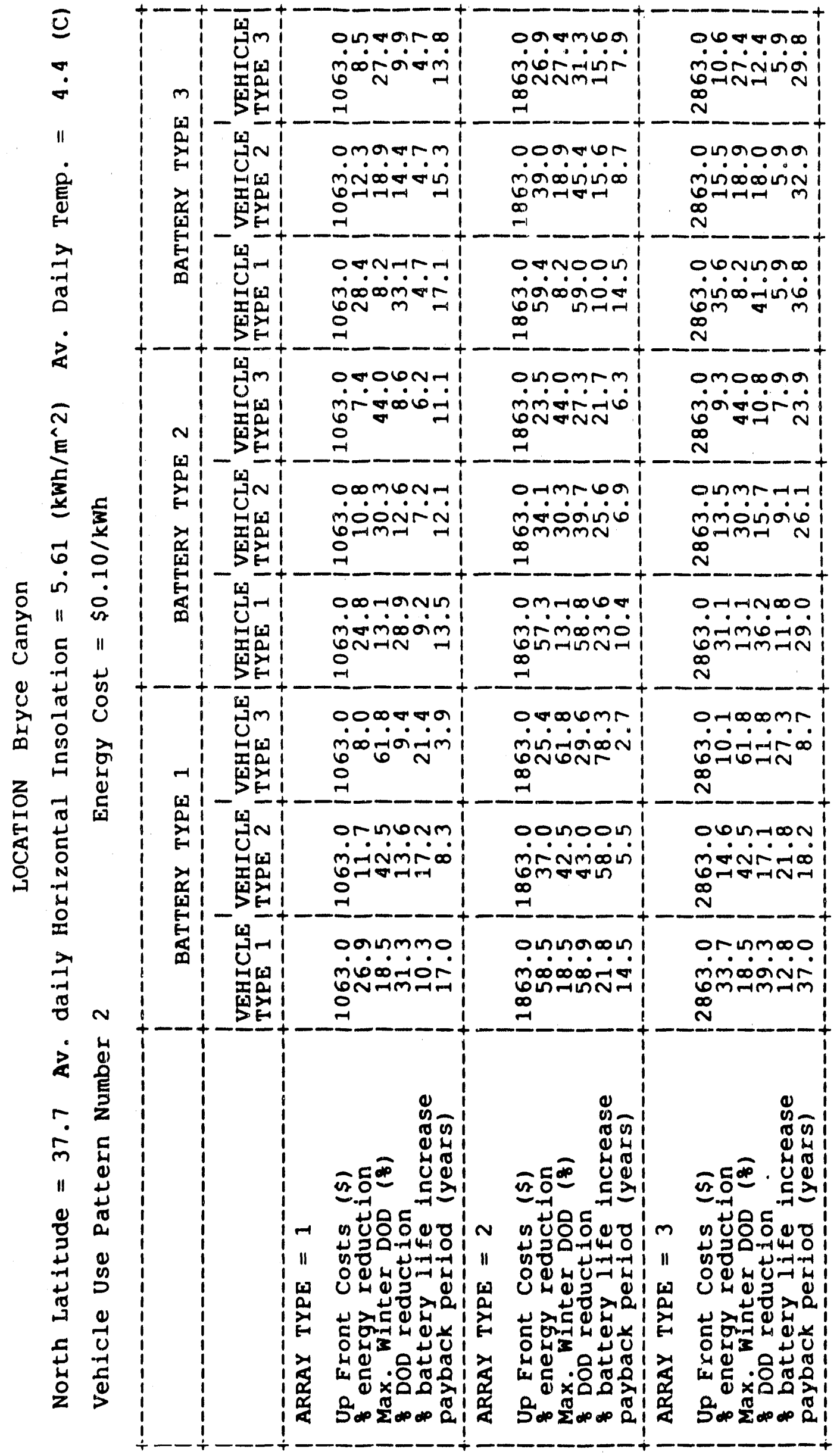




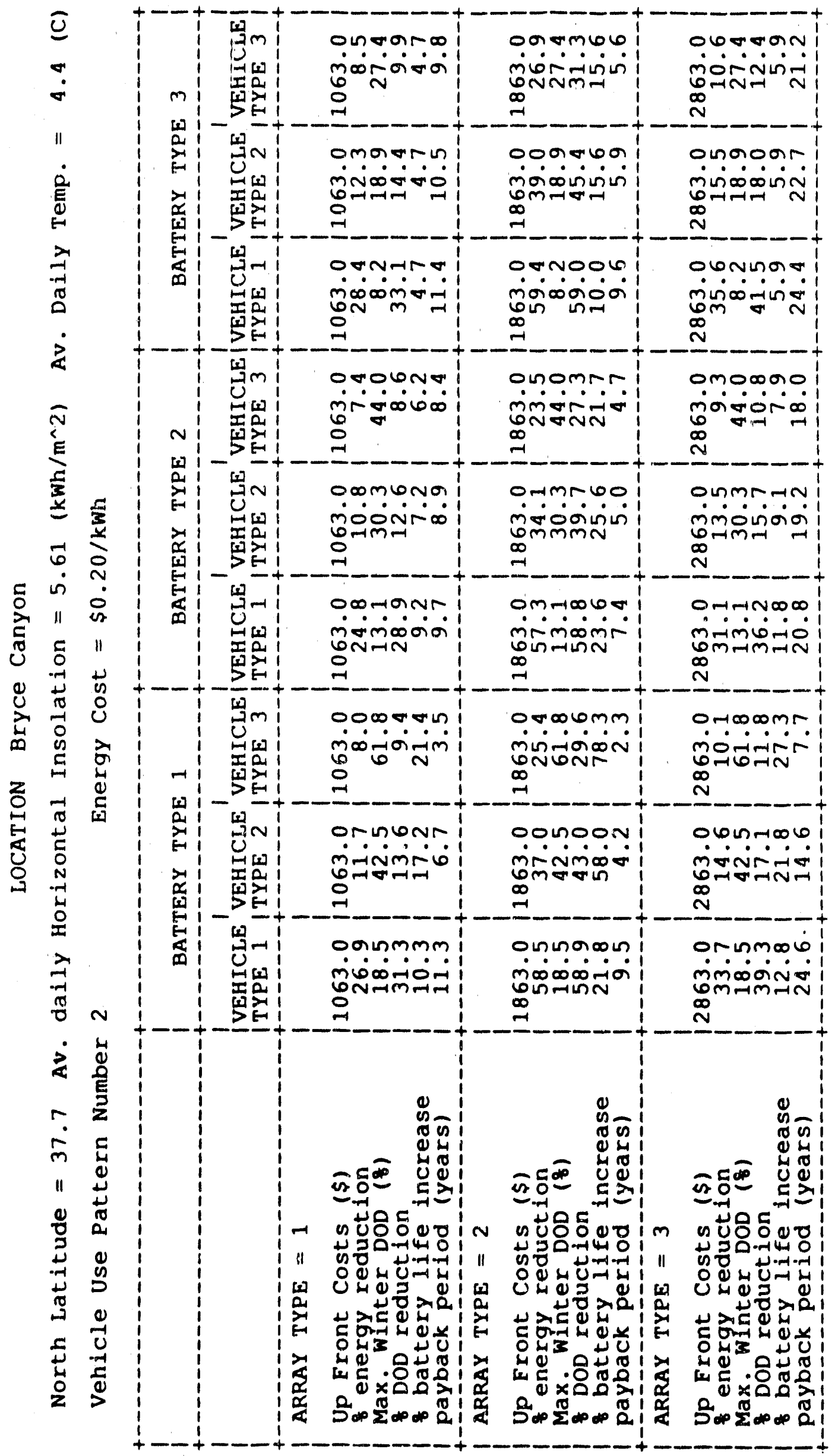




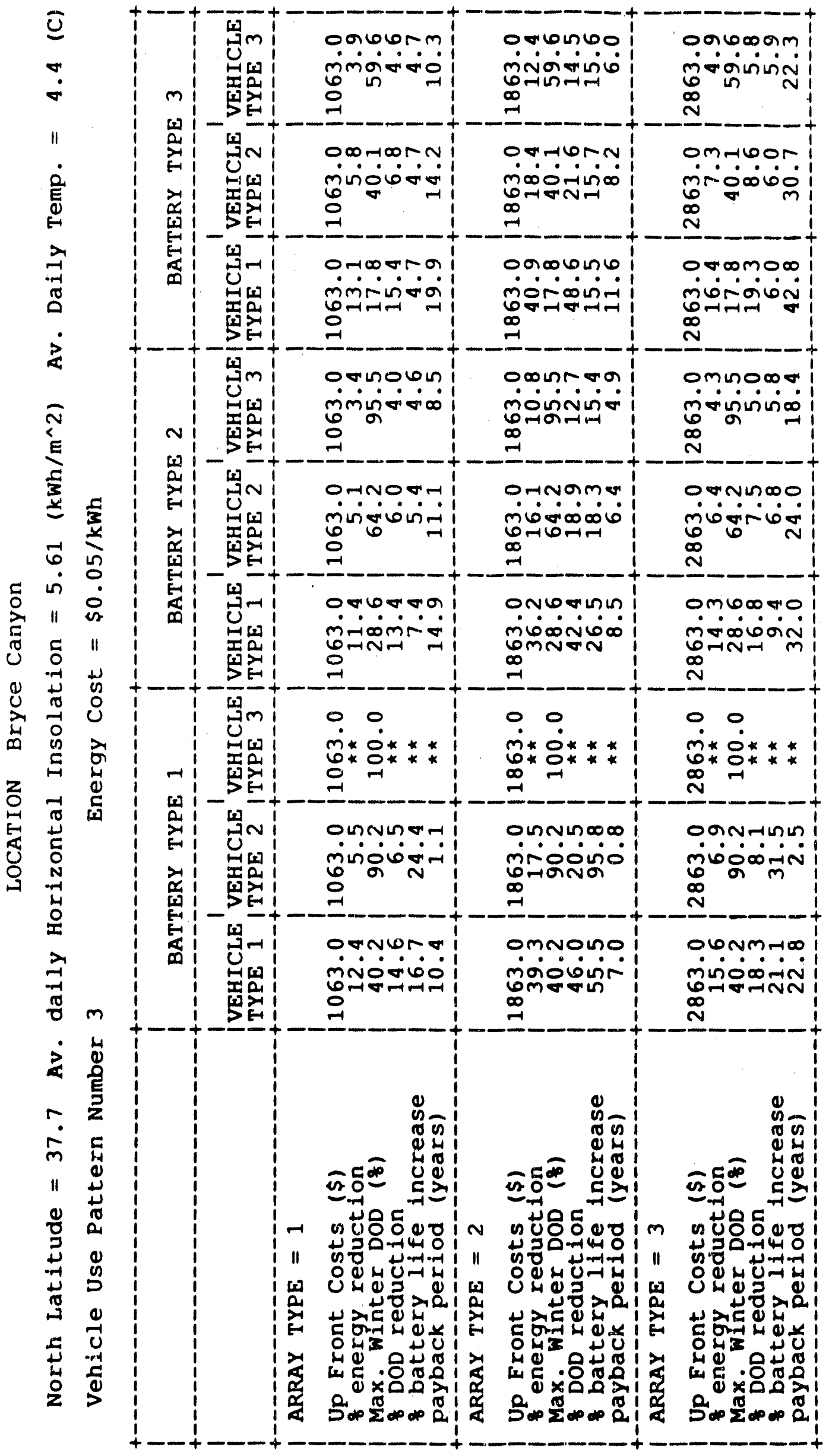




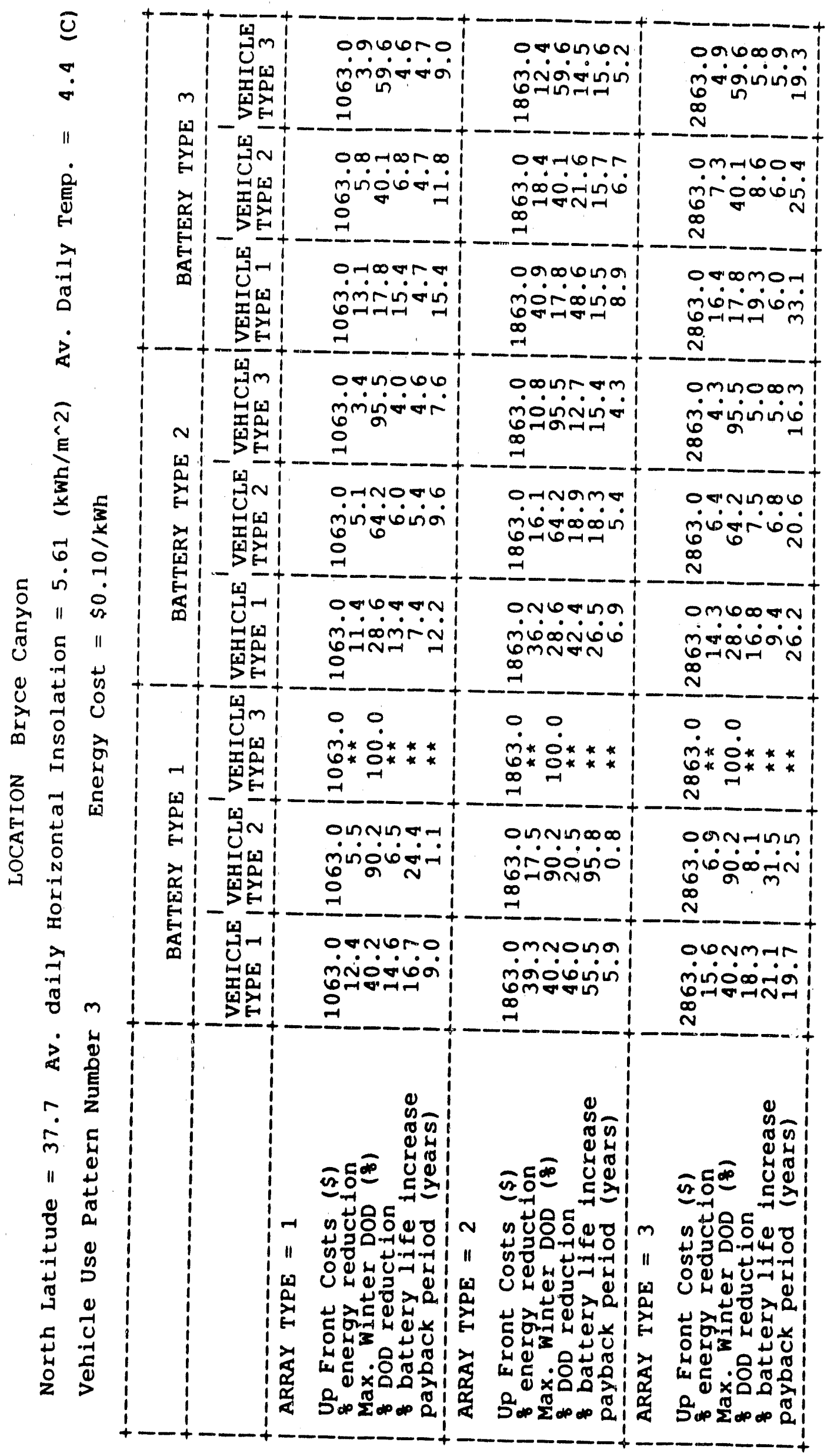




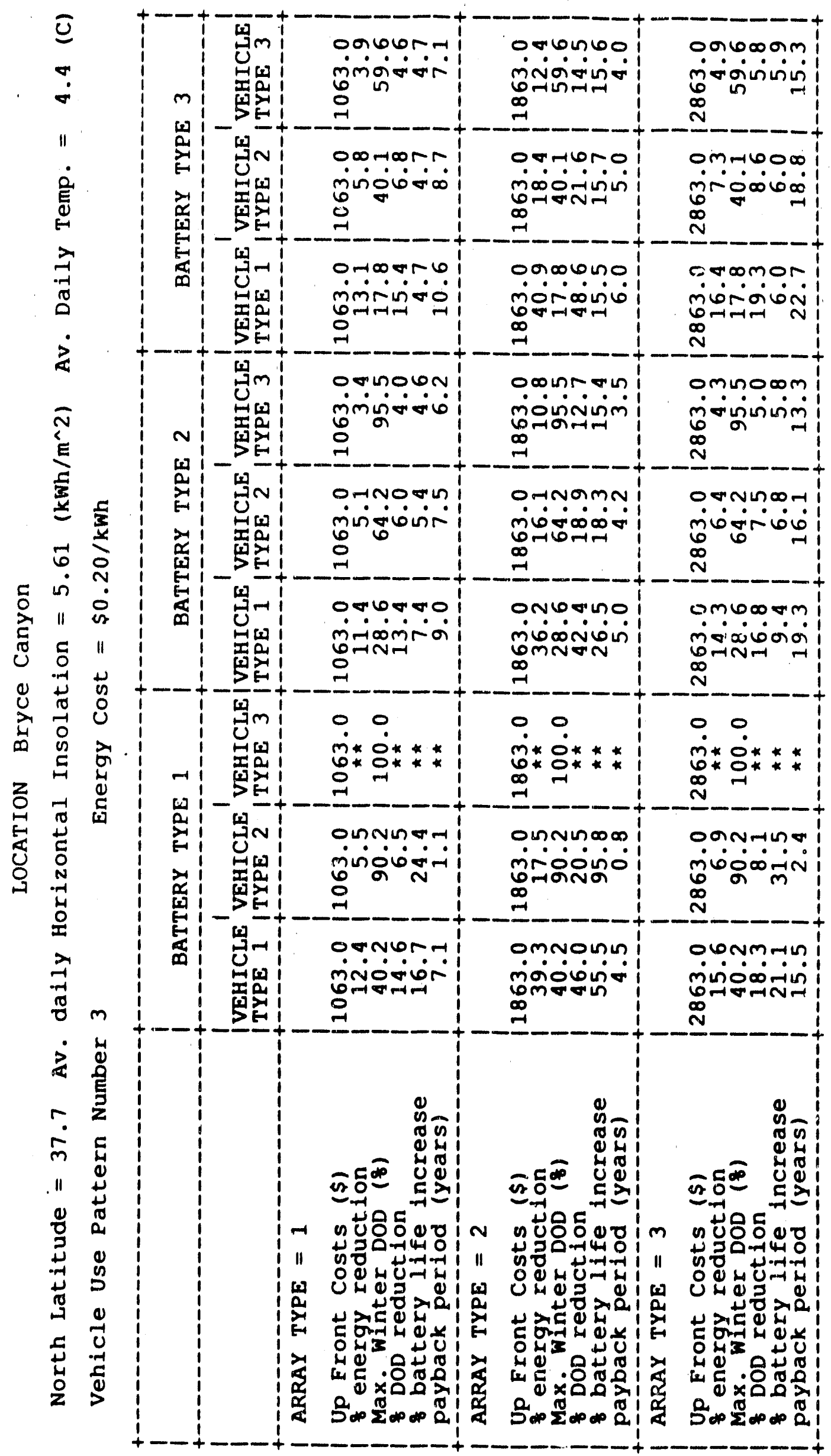




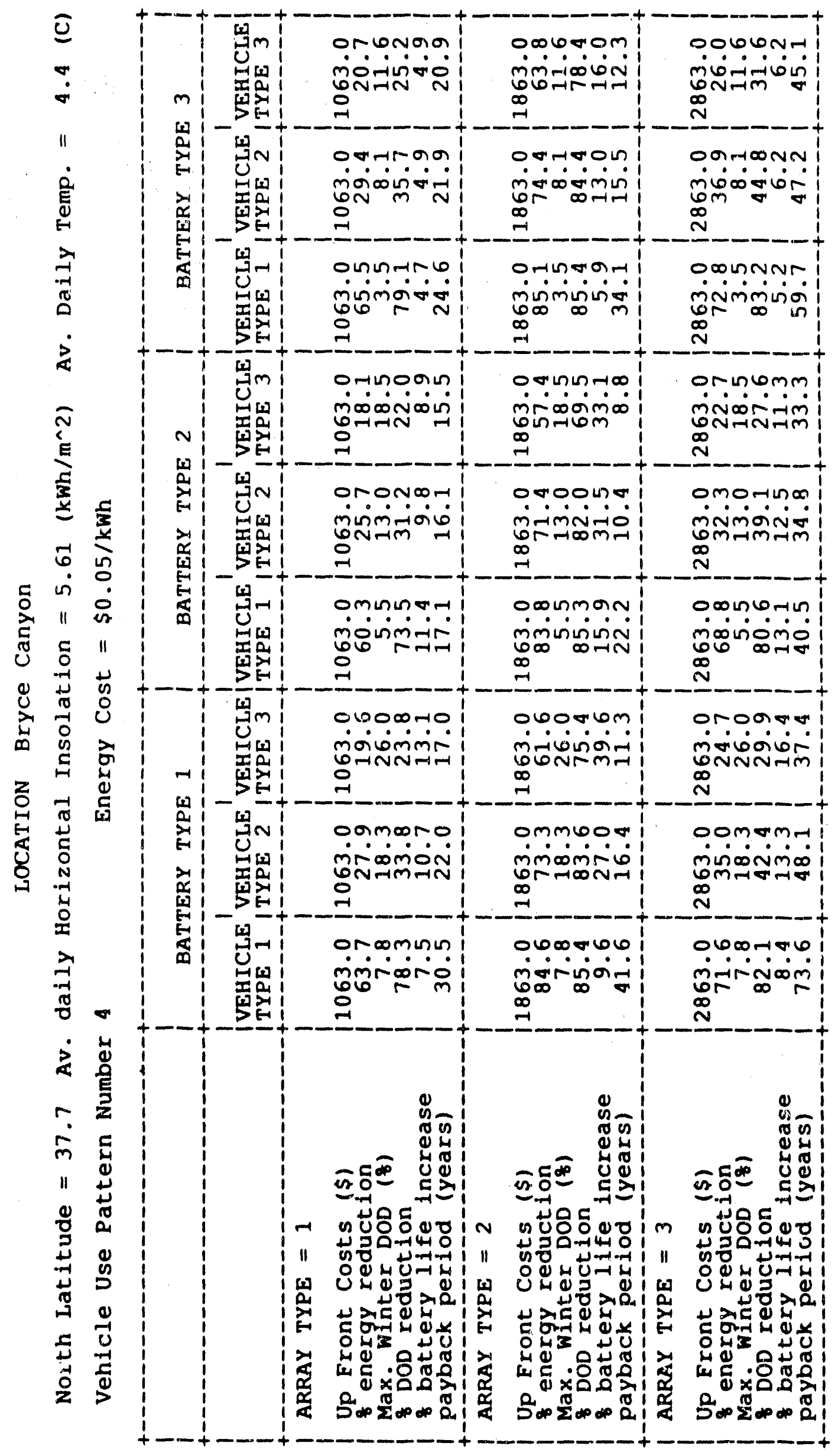




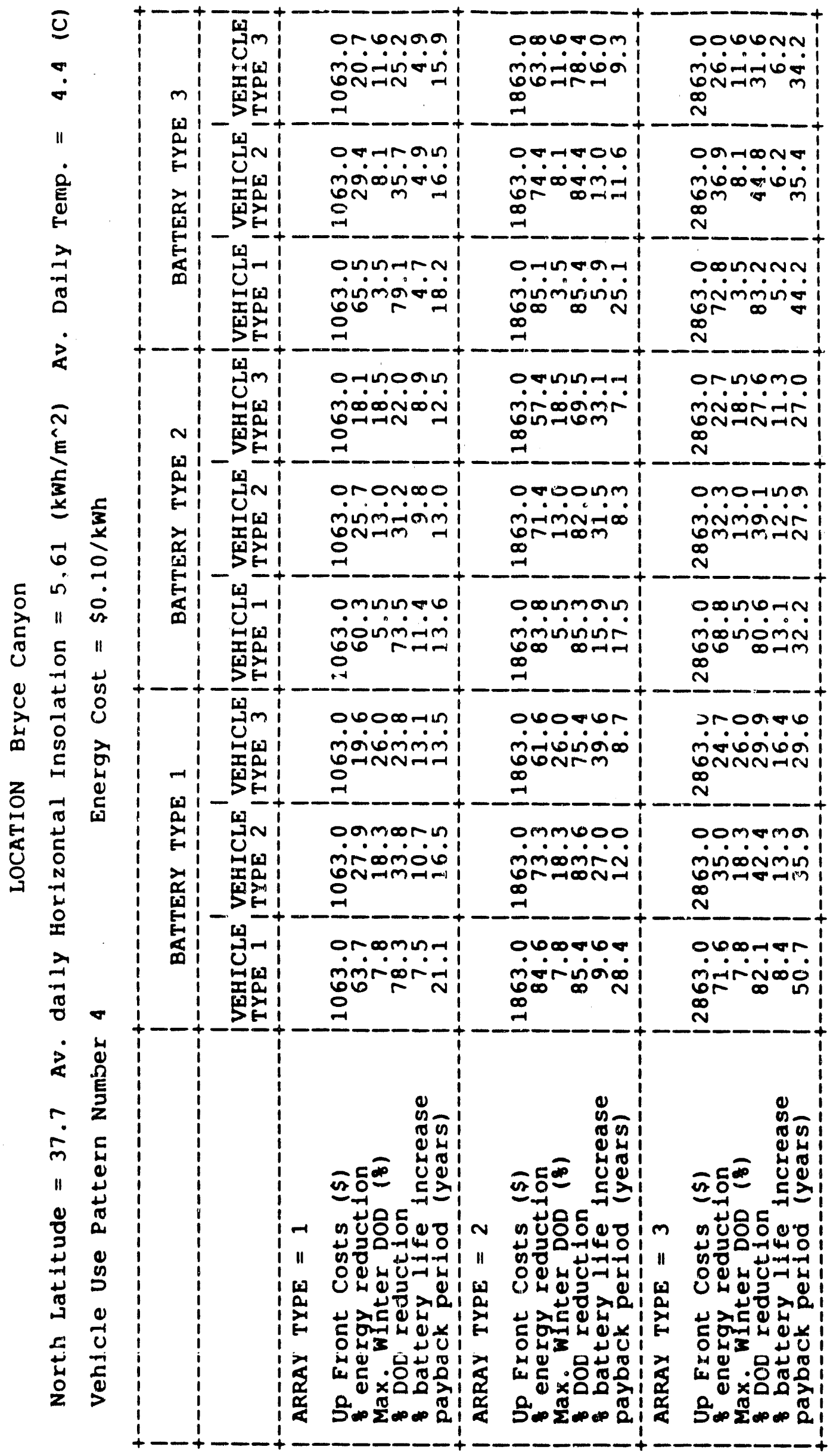




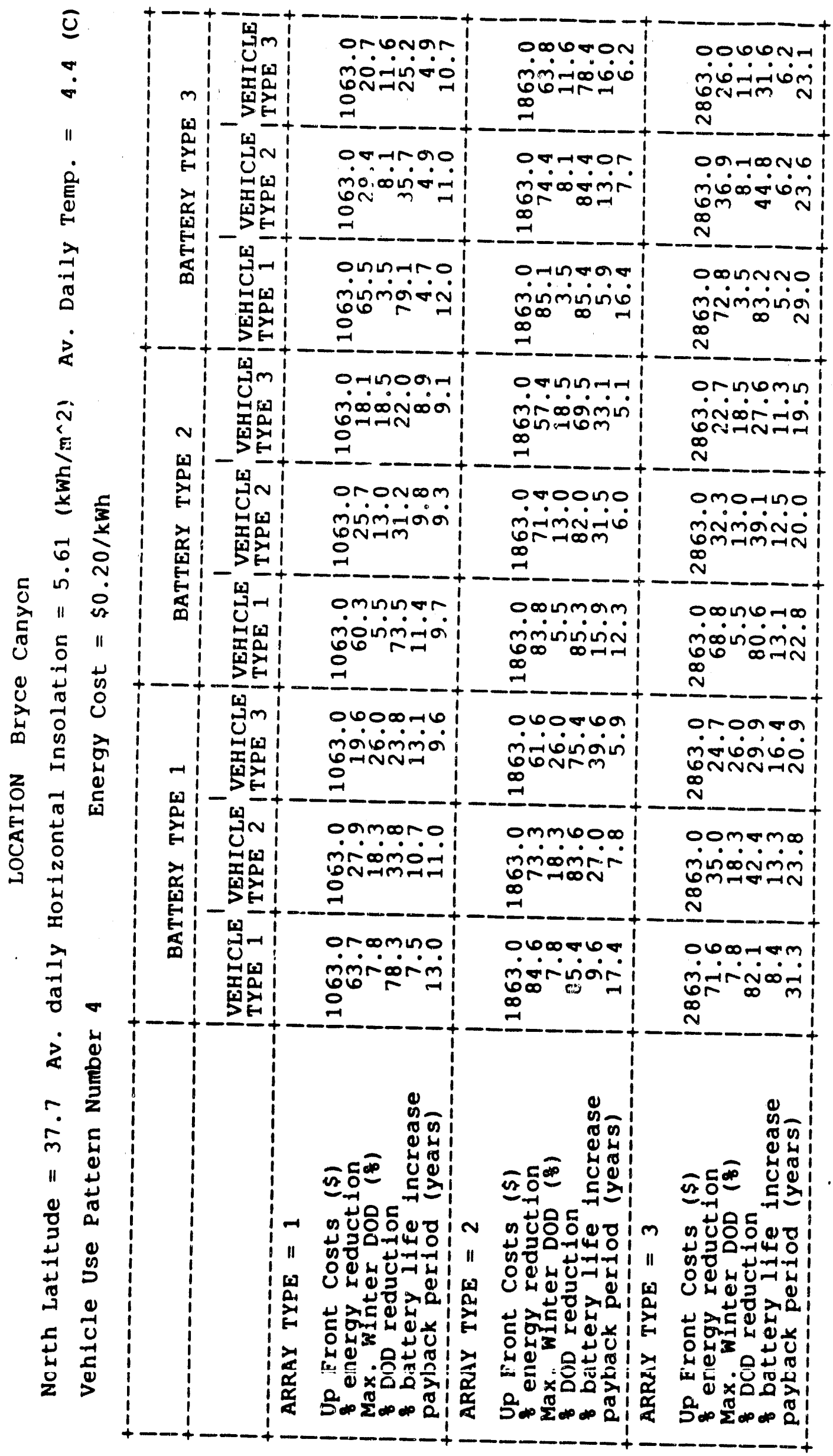




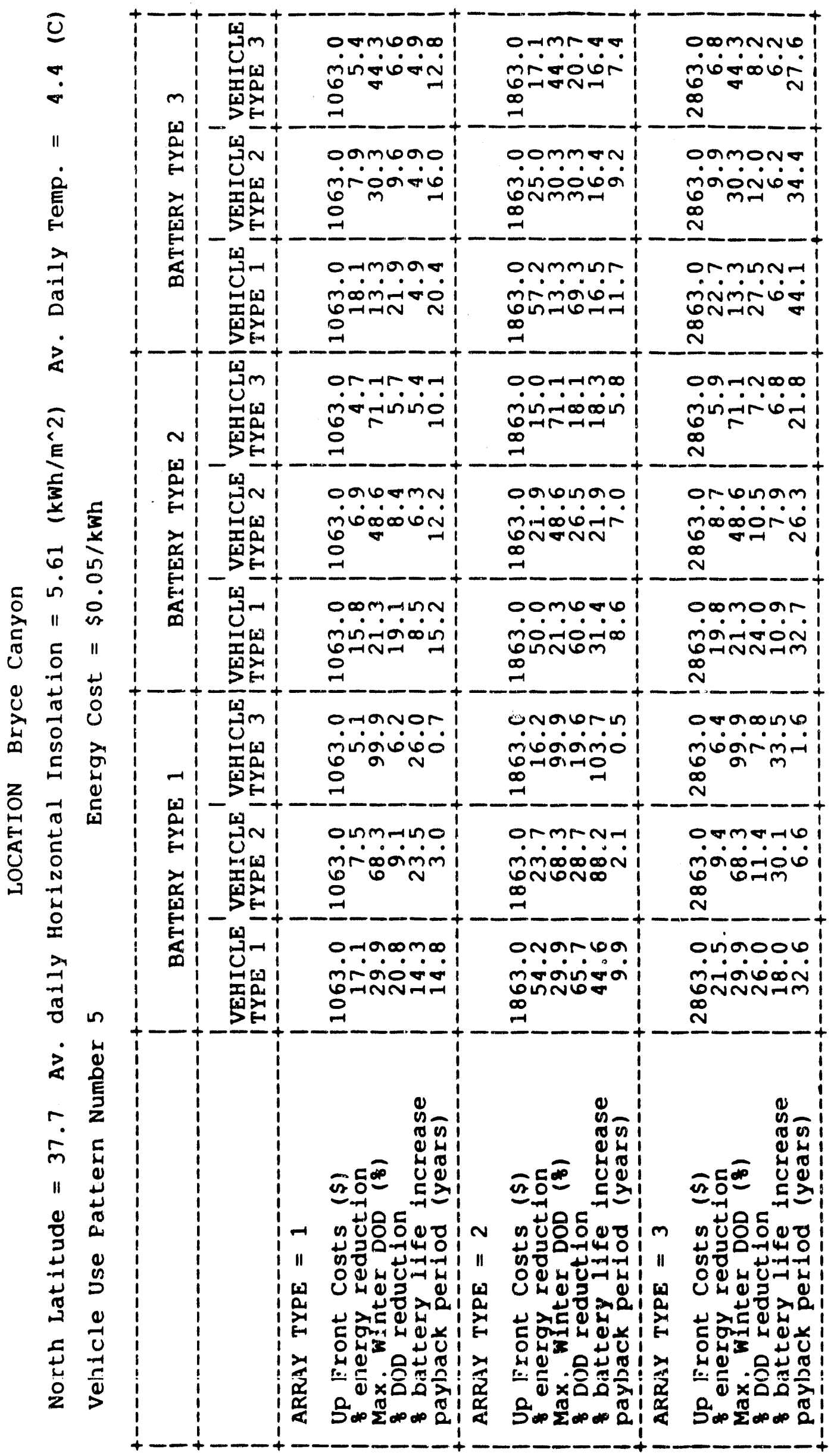




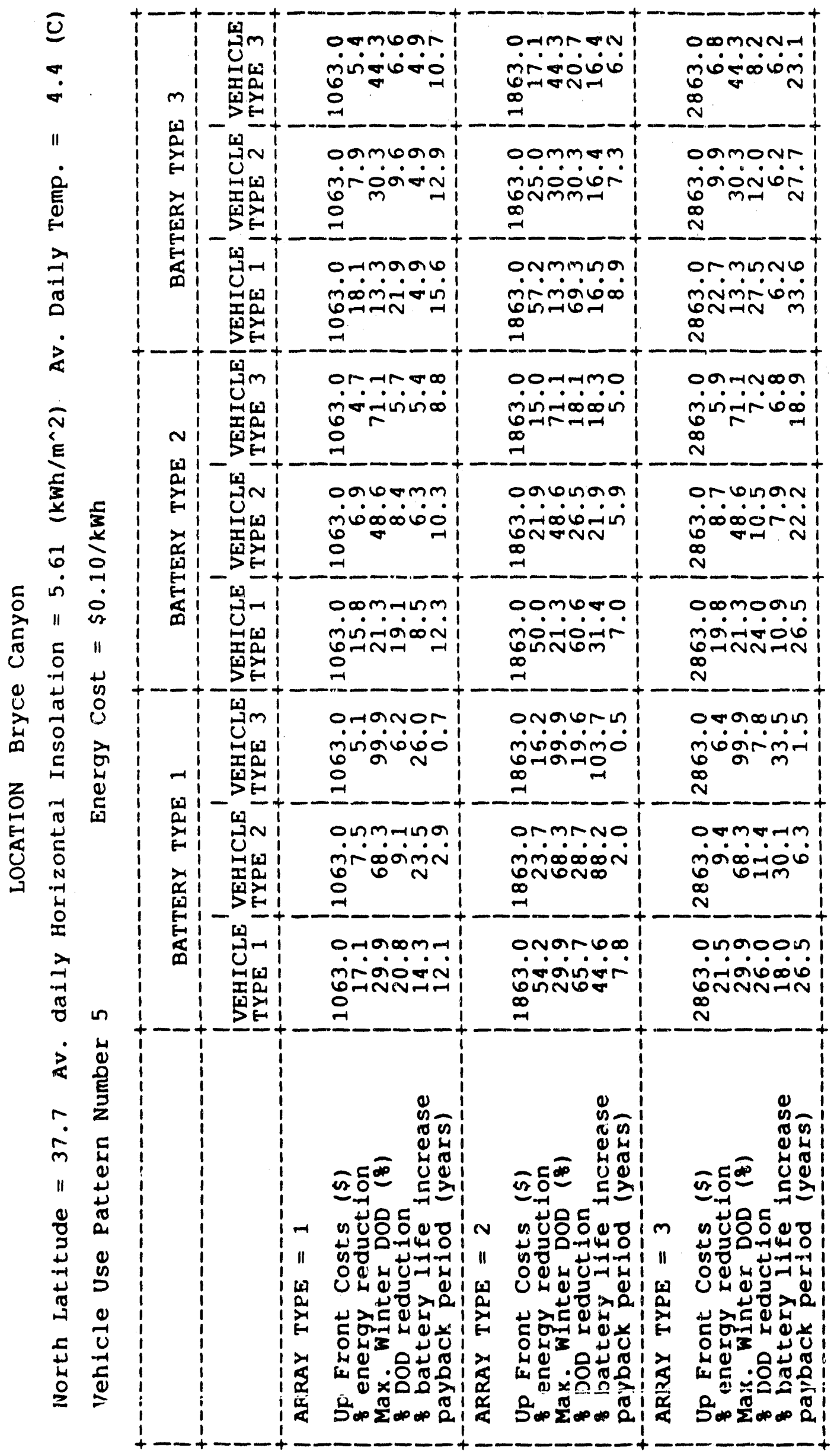




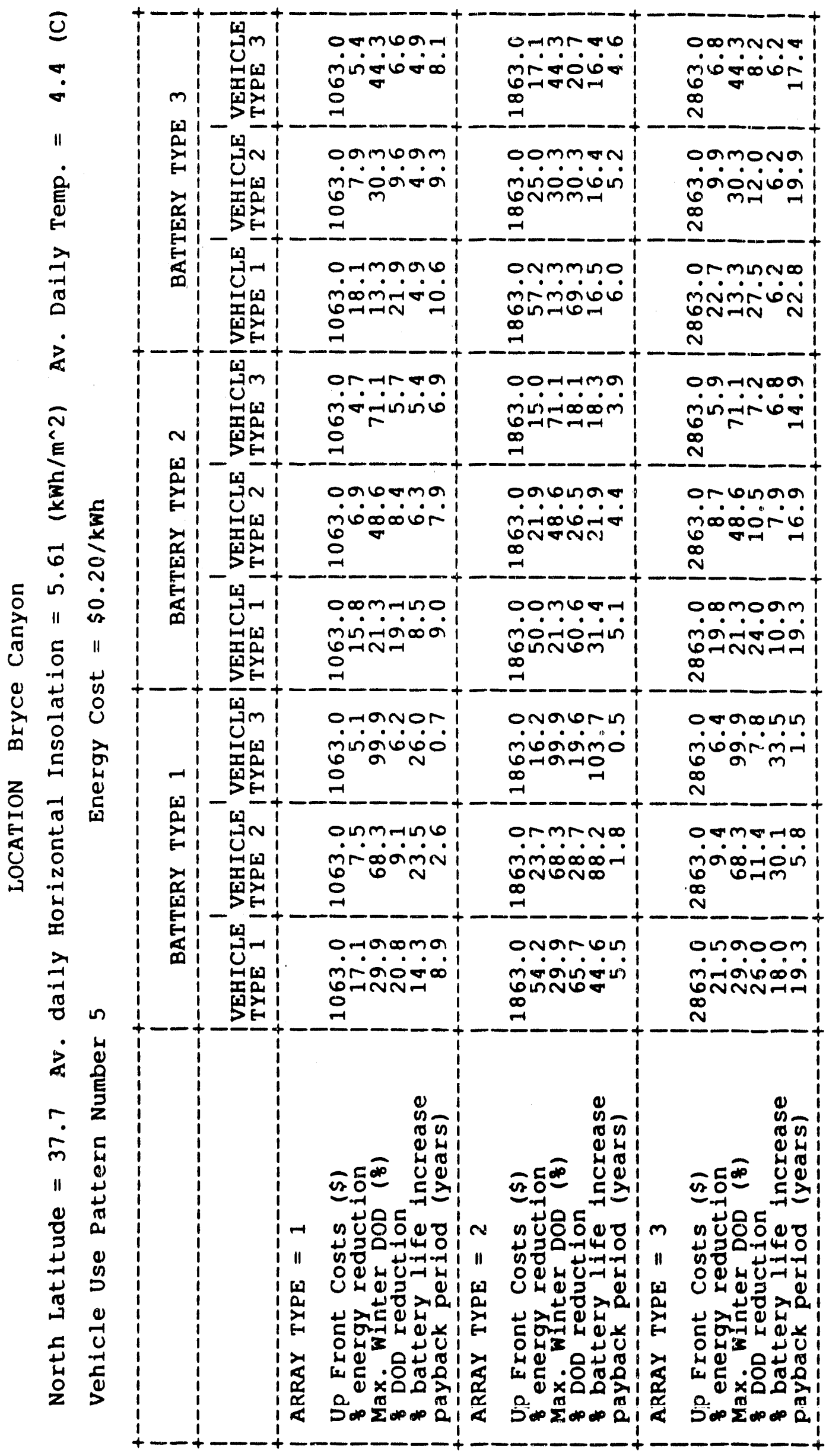




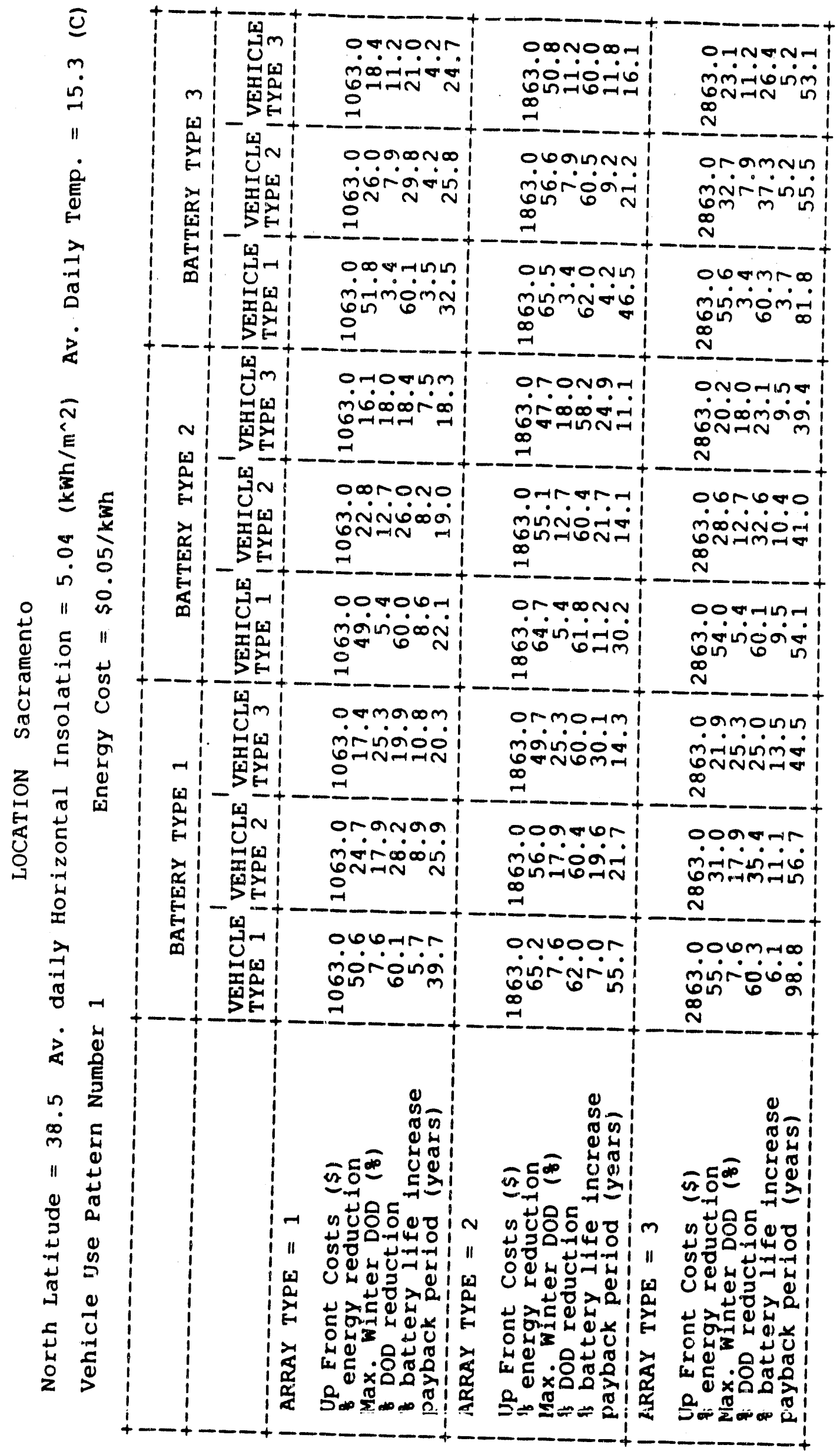

$\stackrel{1}{n}$ 


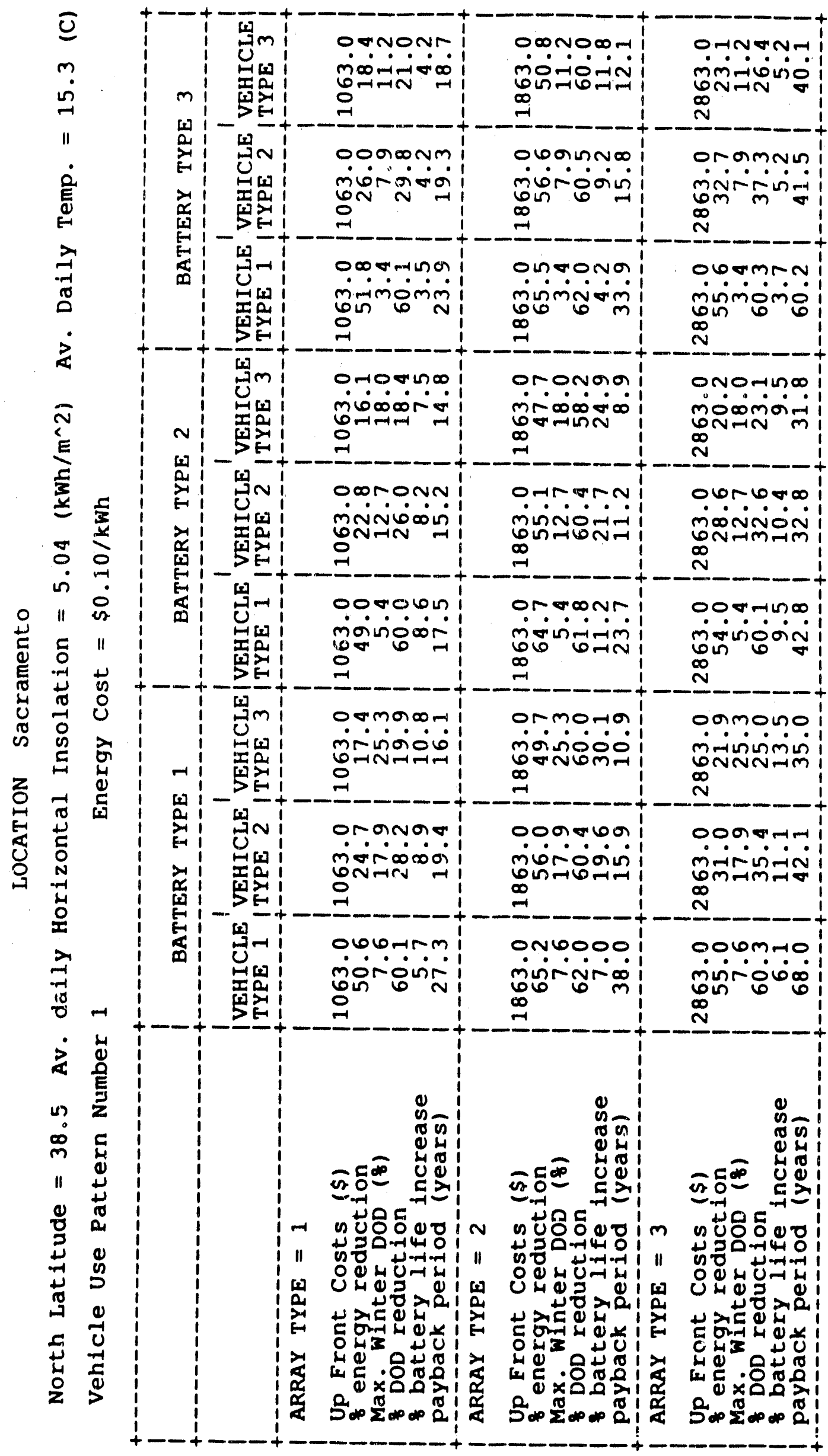




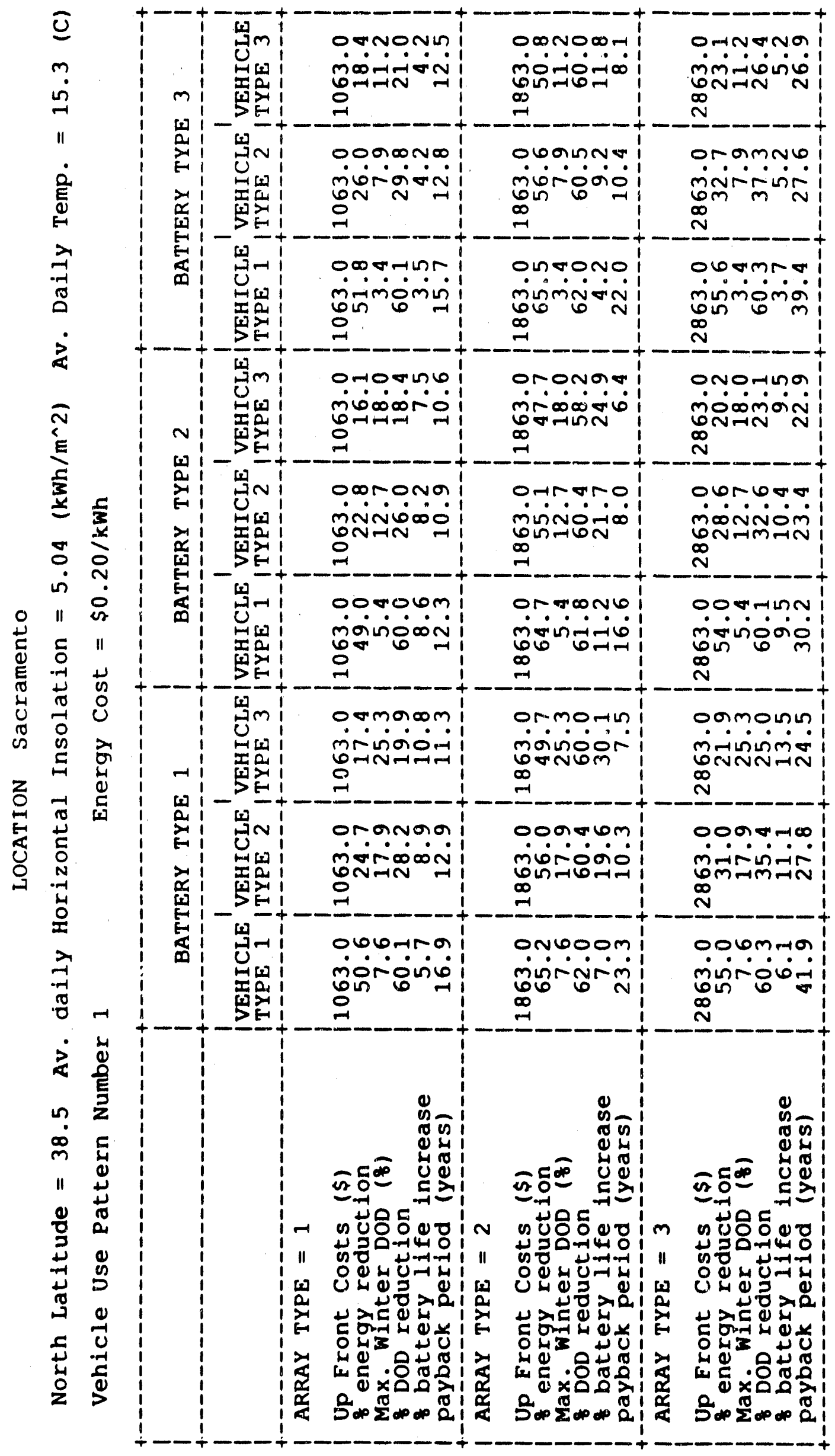




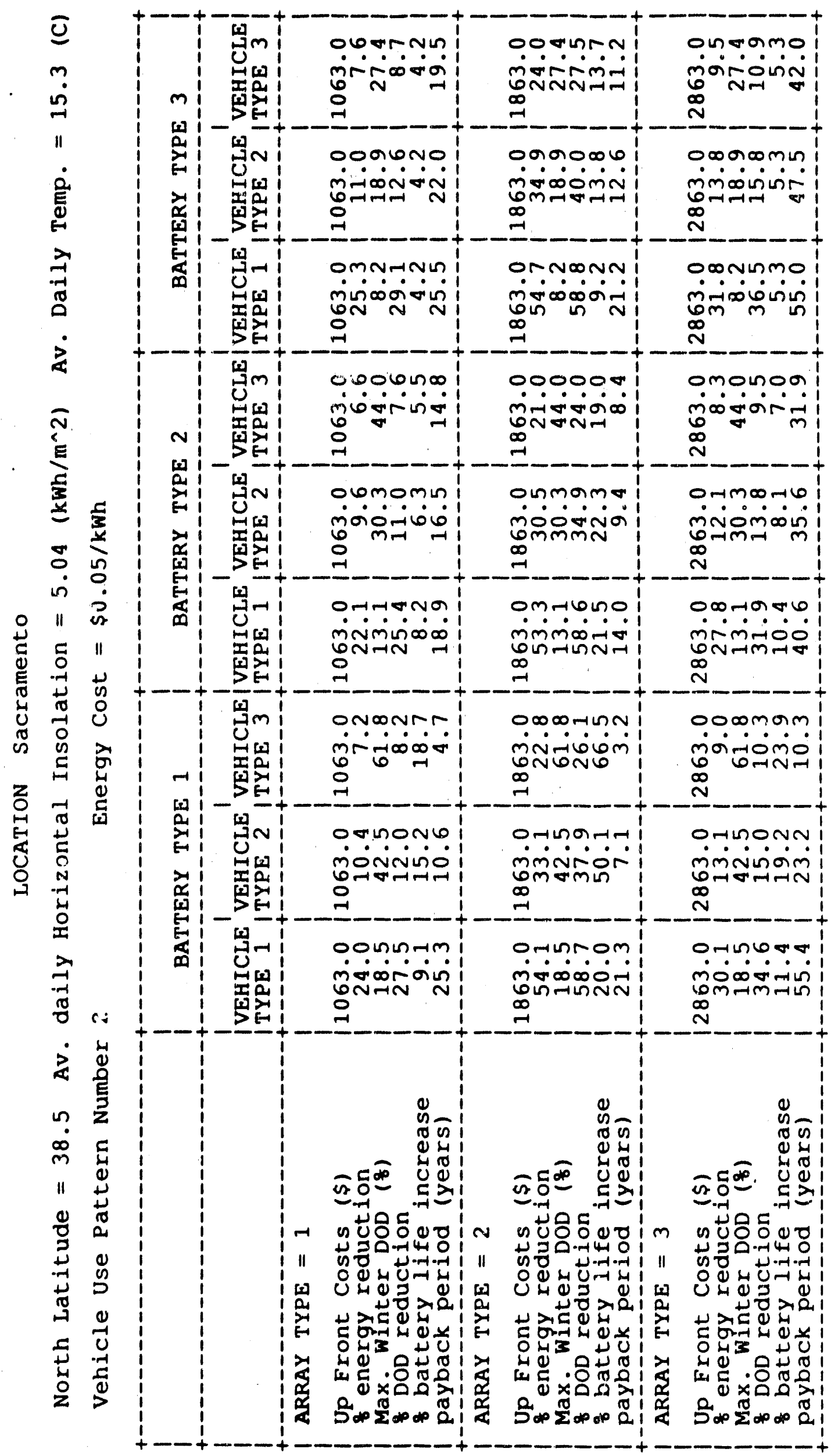




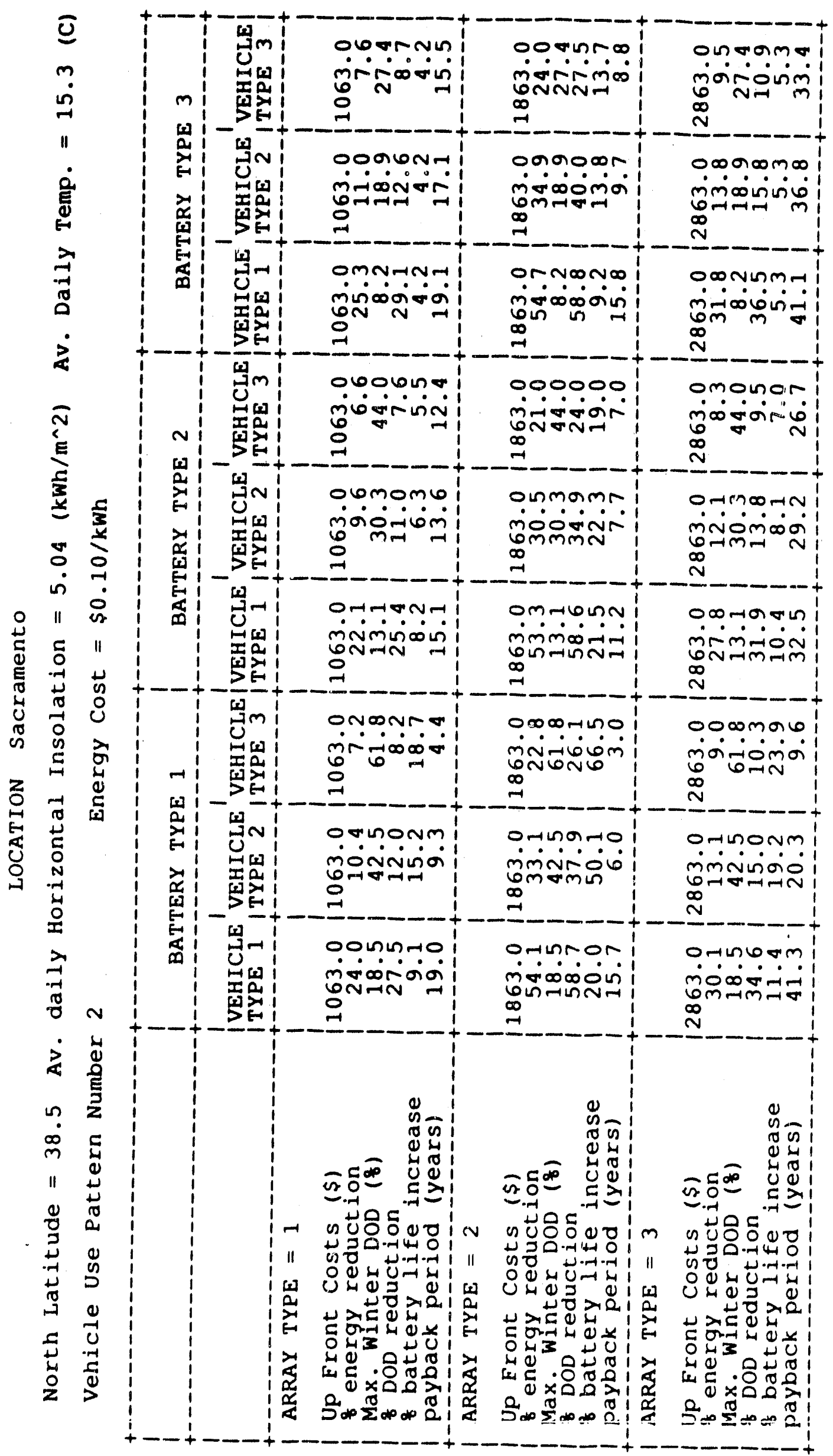




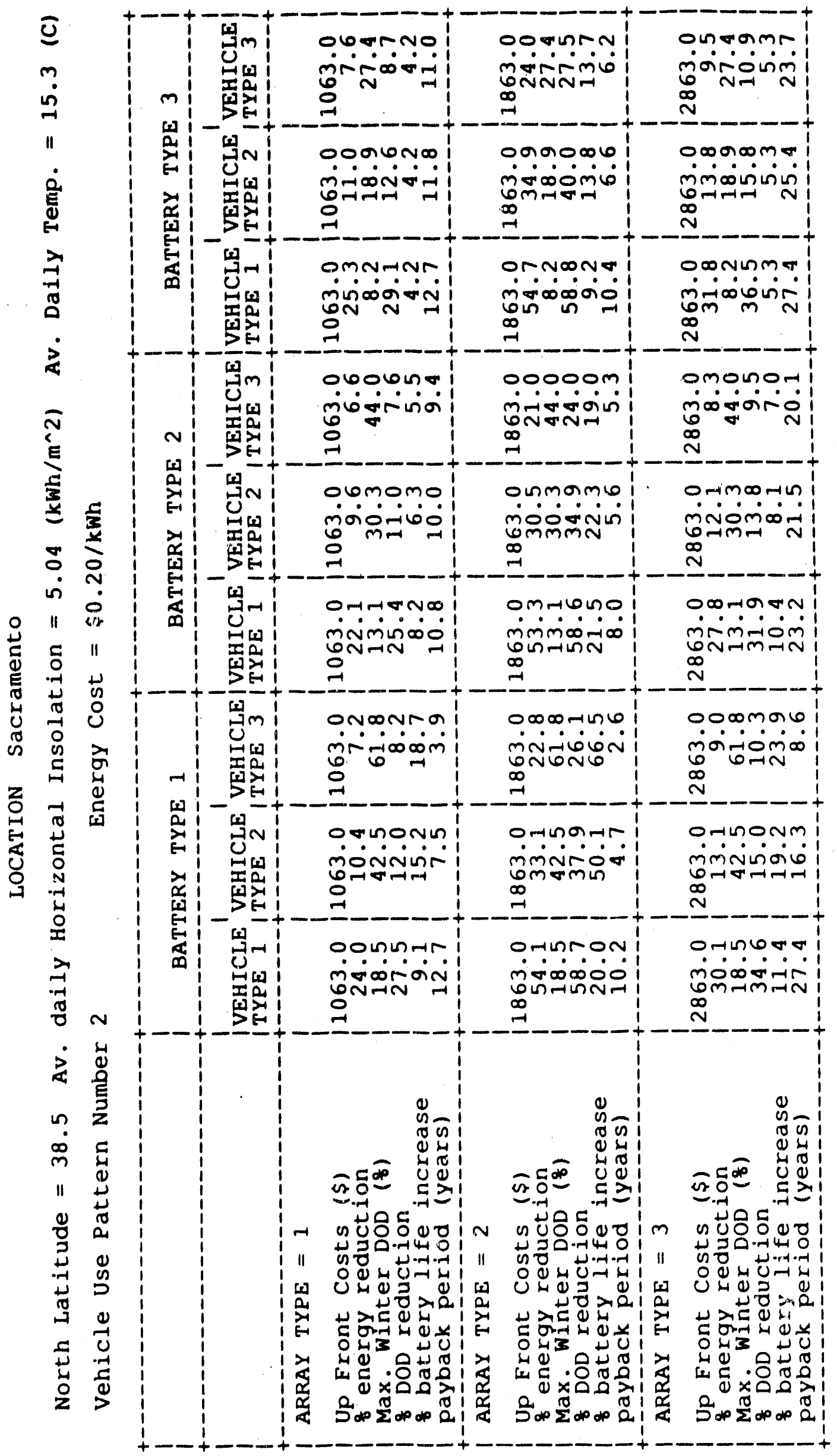




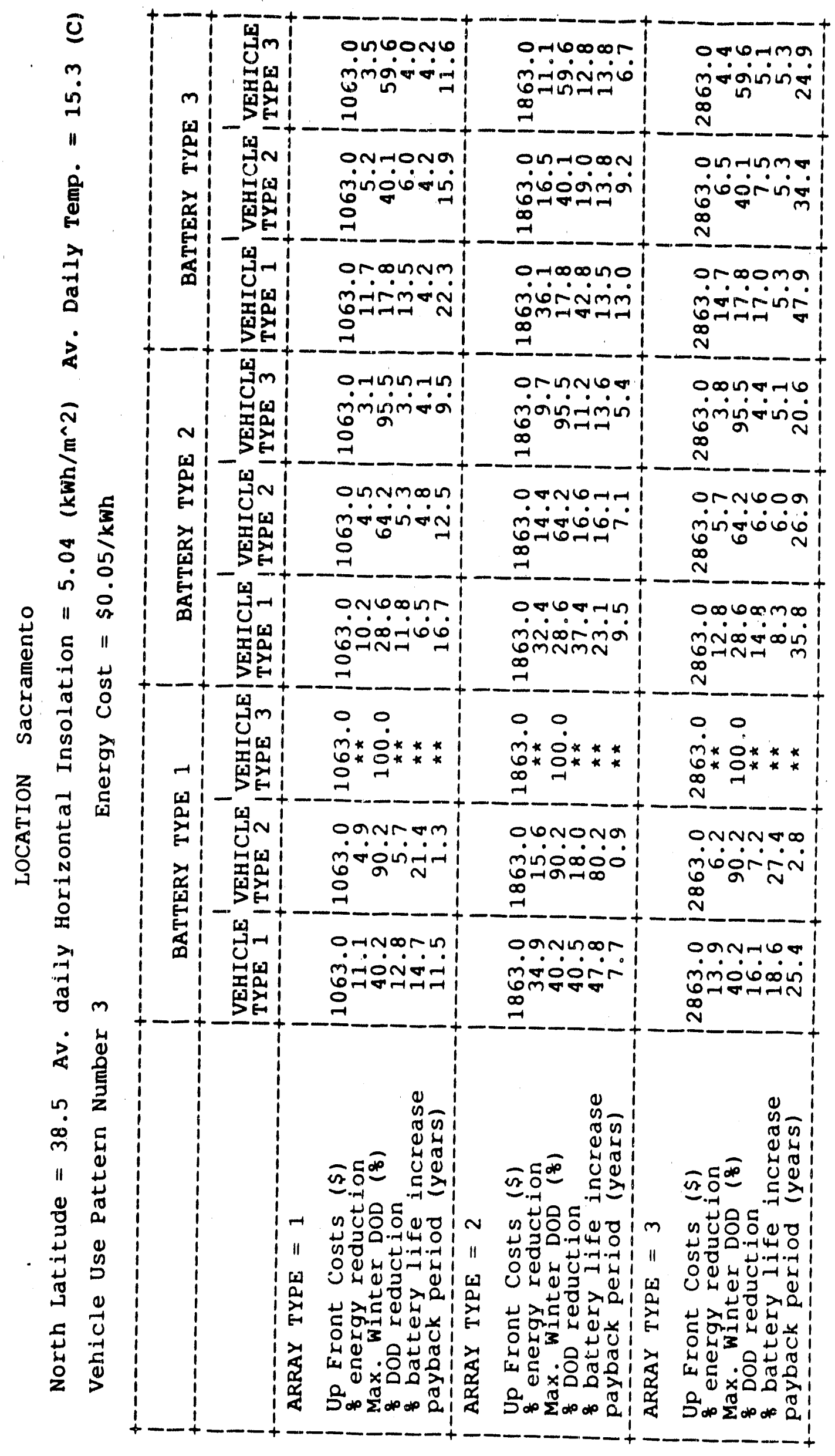




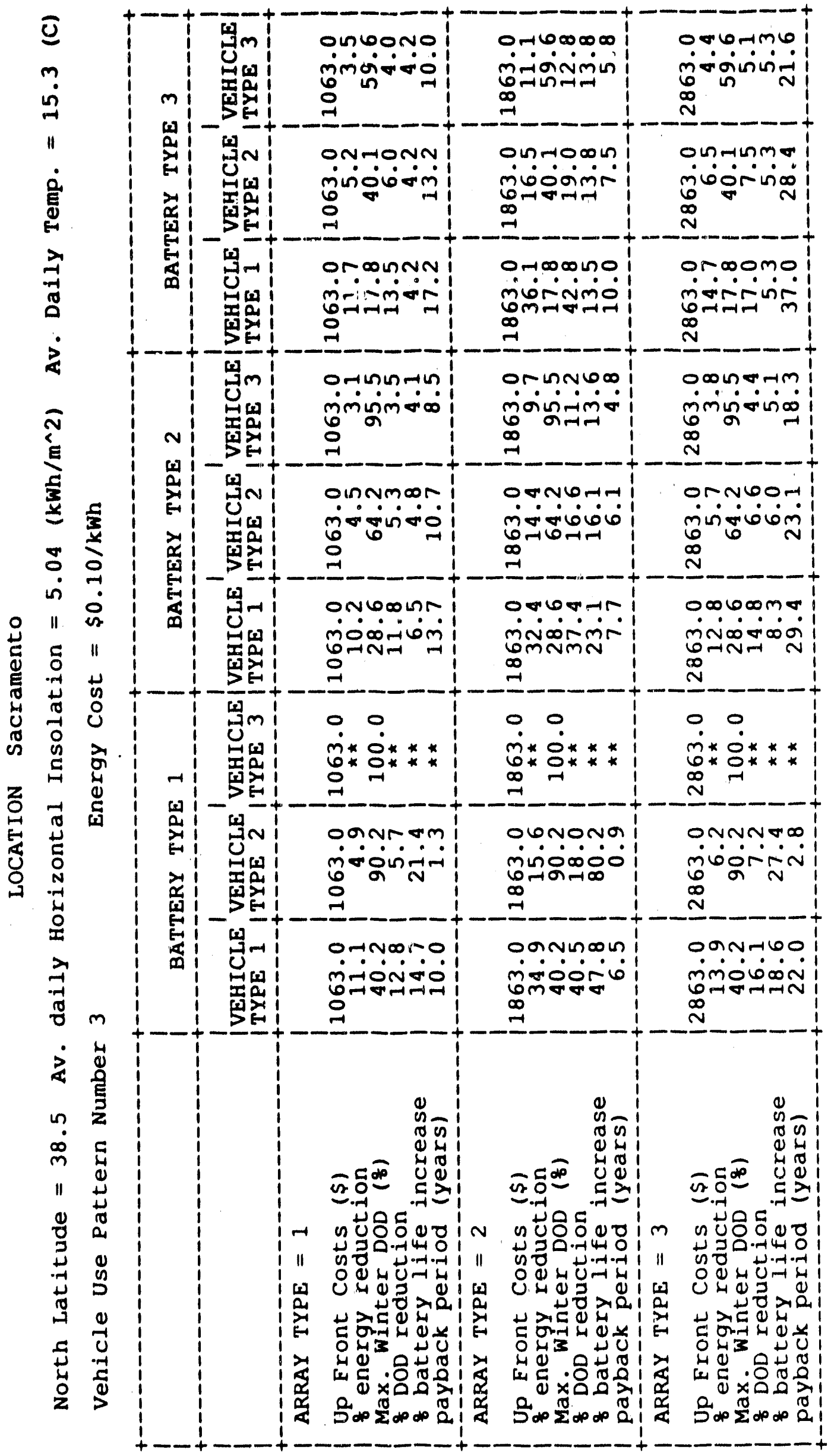




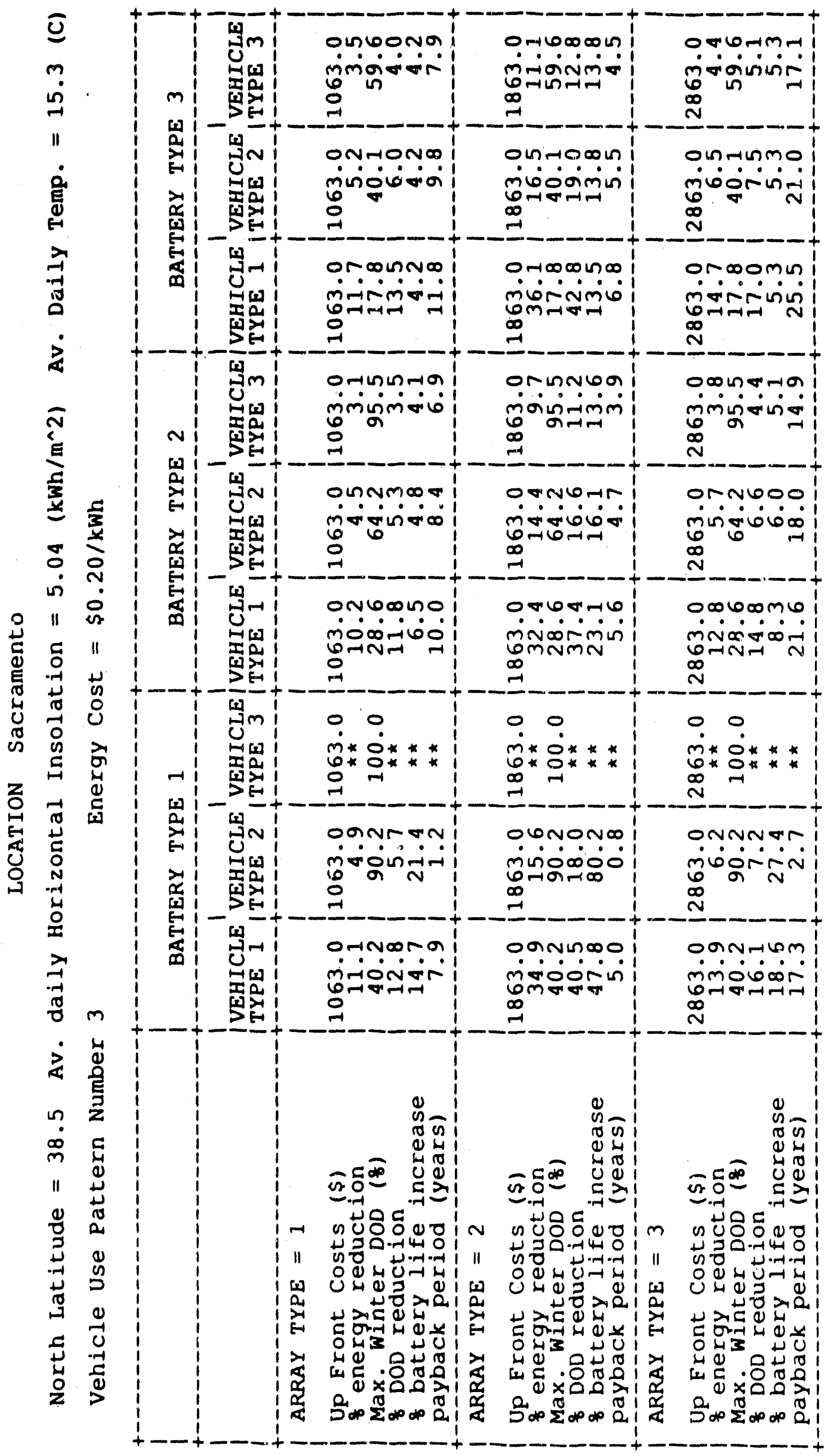




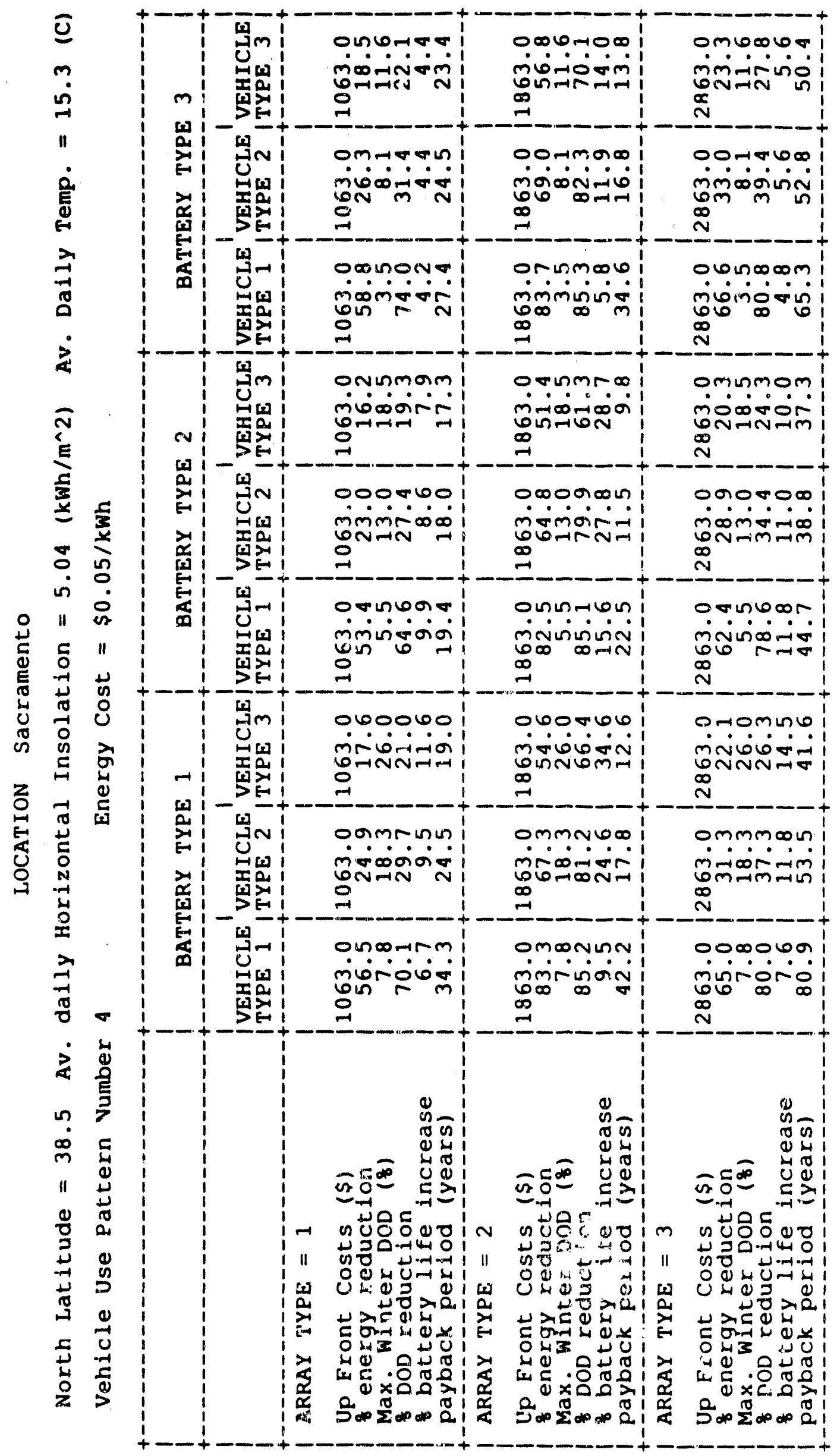




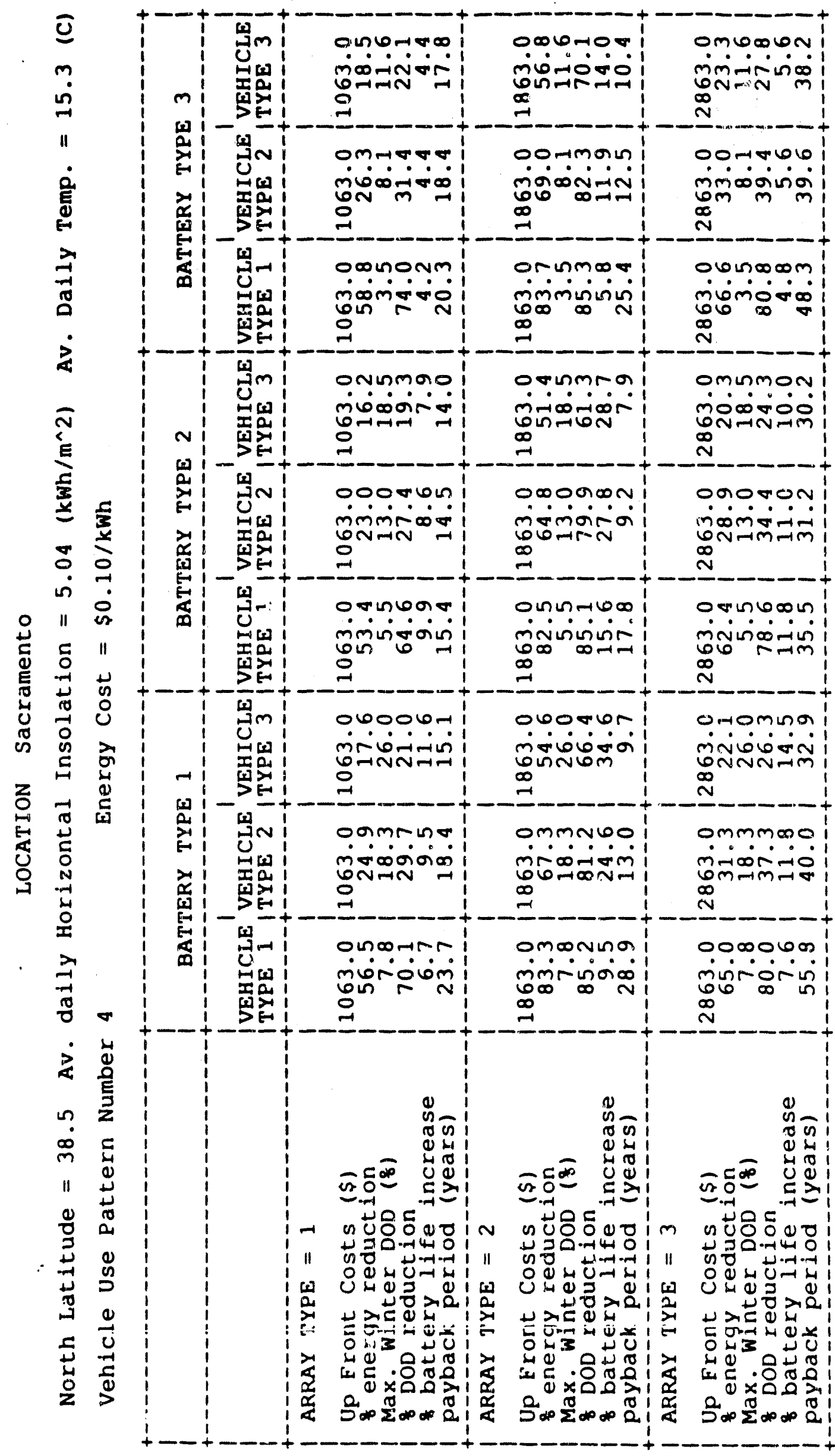




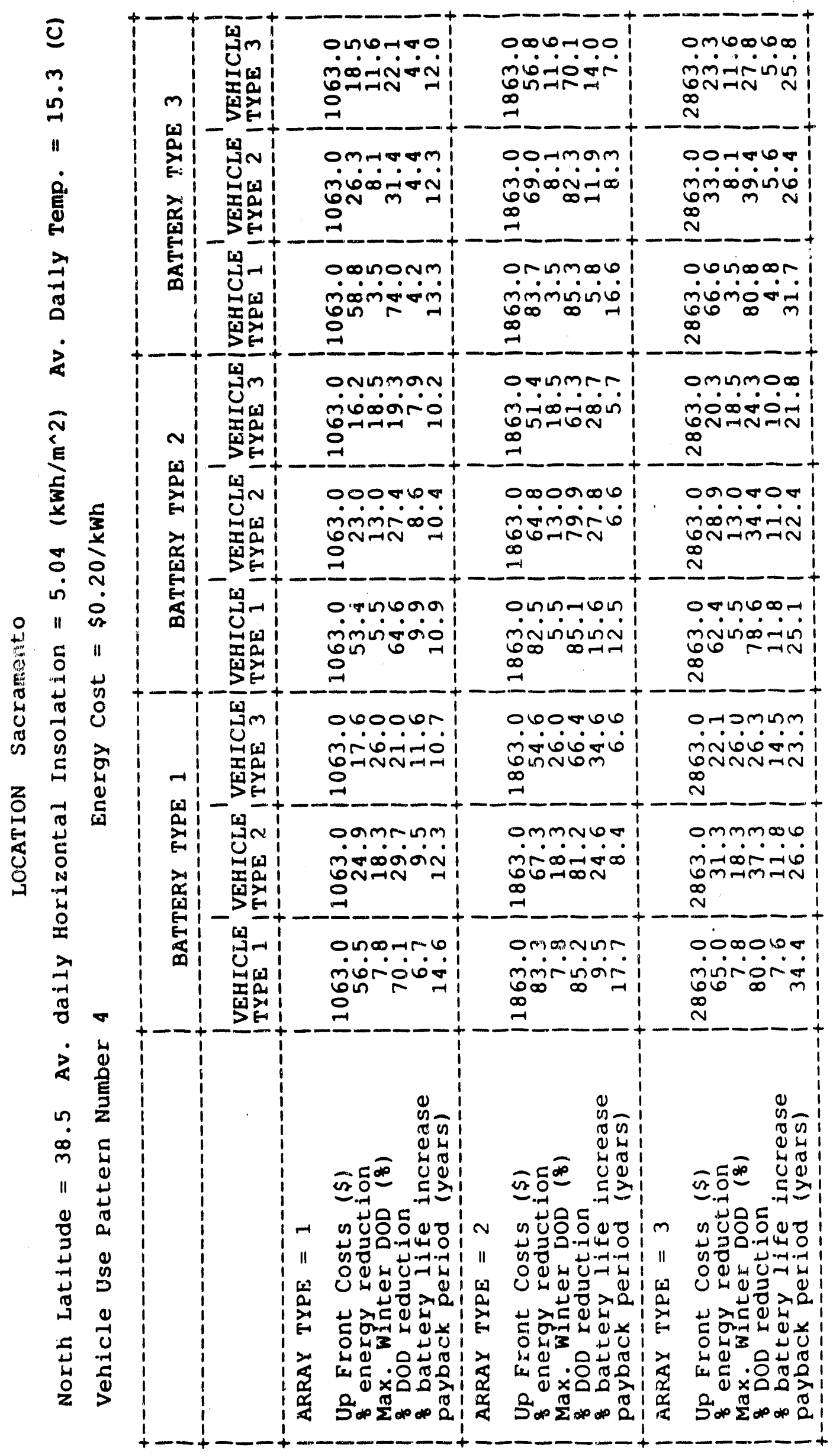




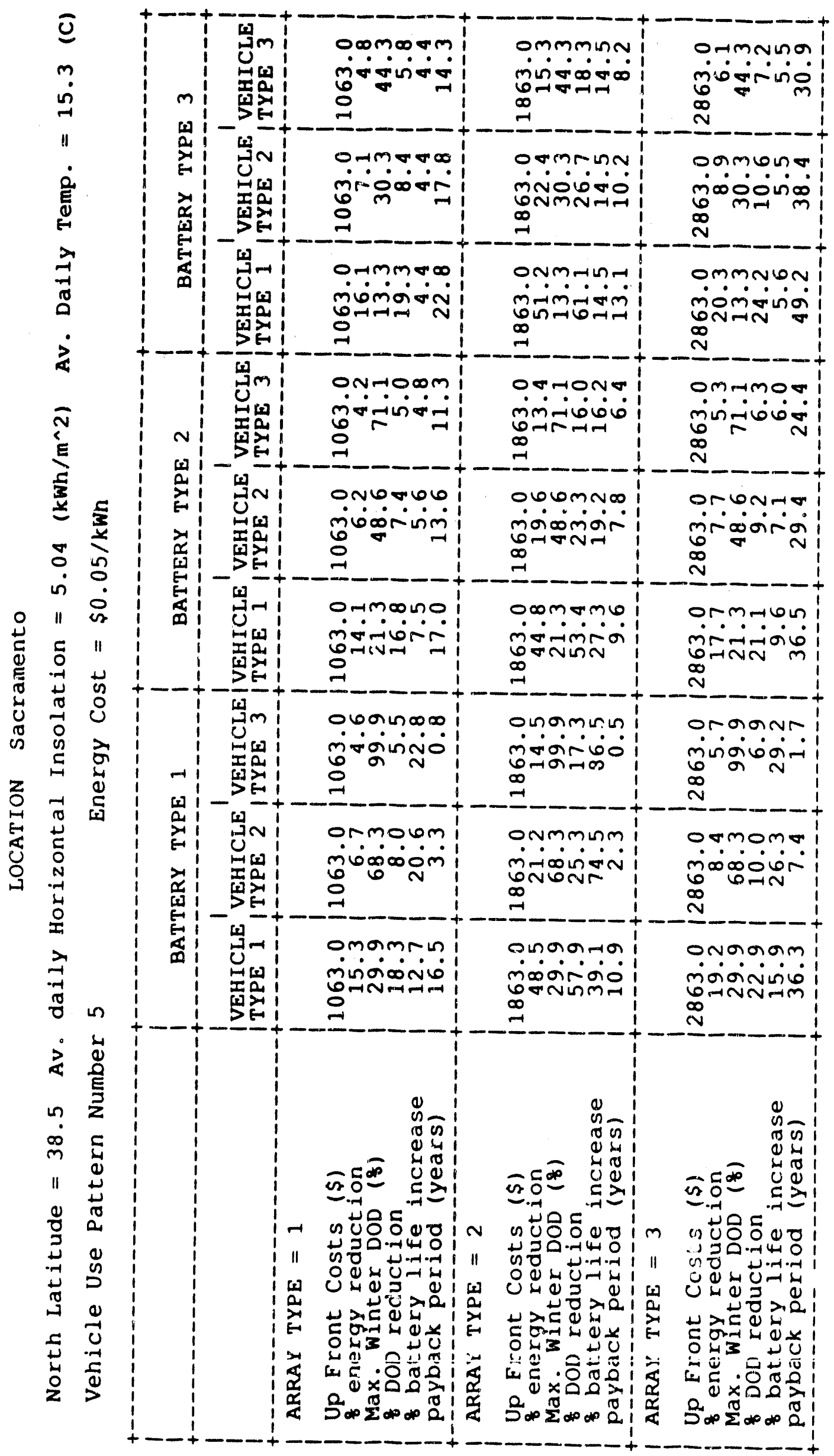




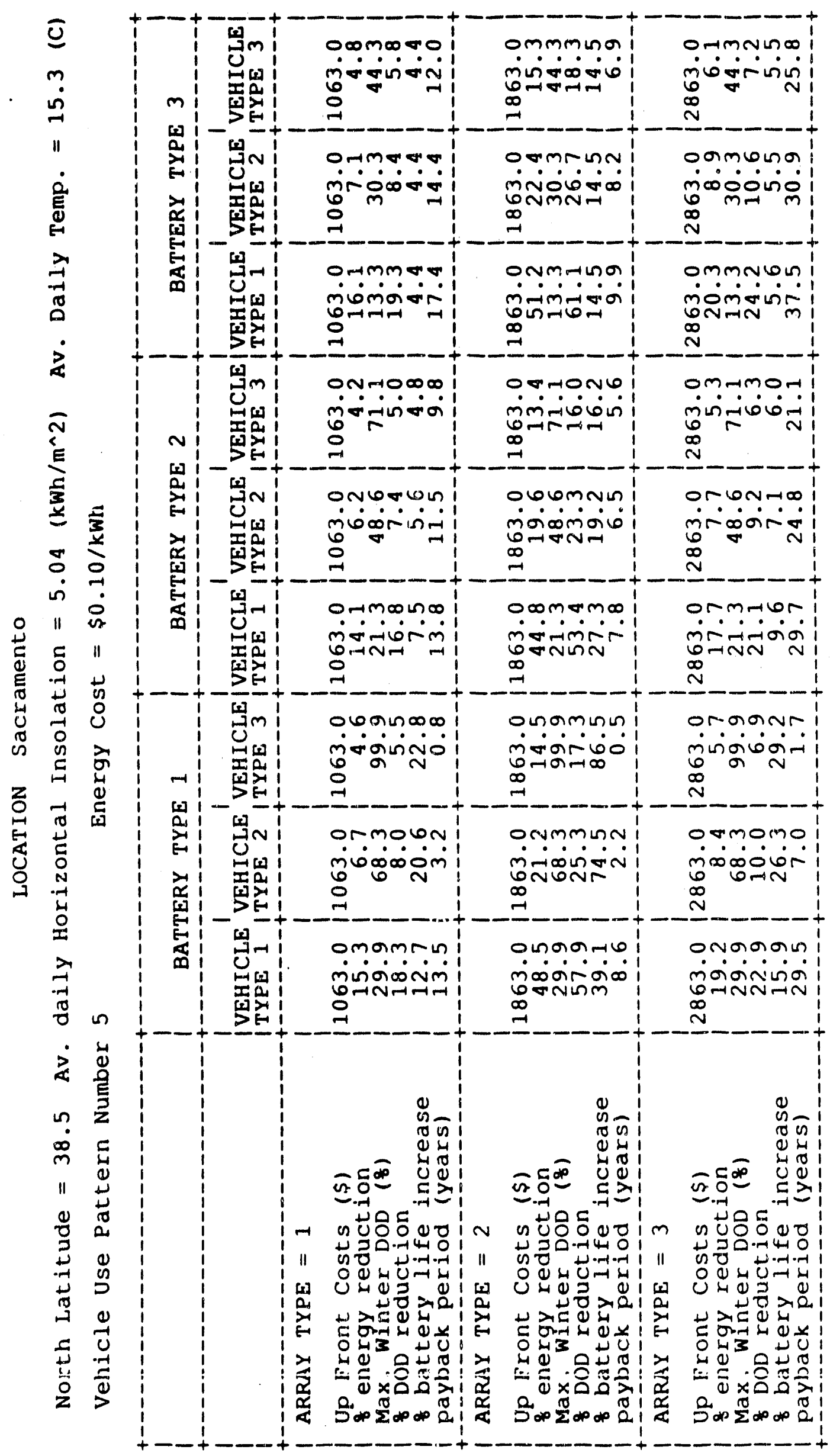




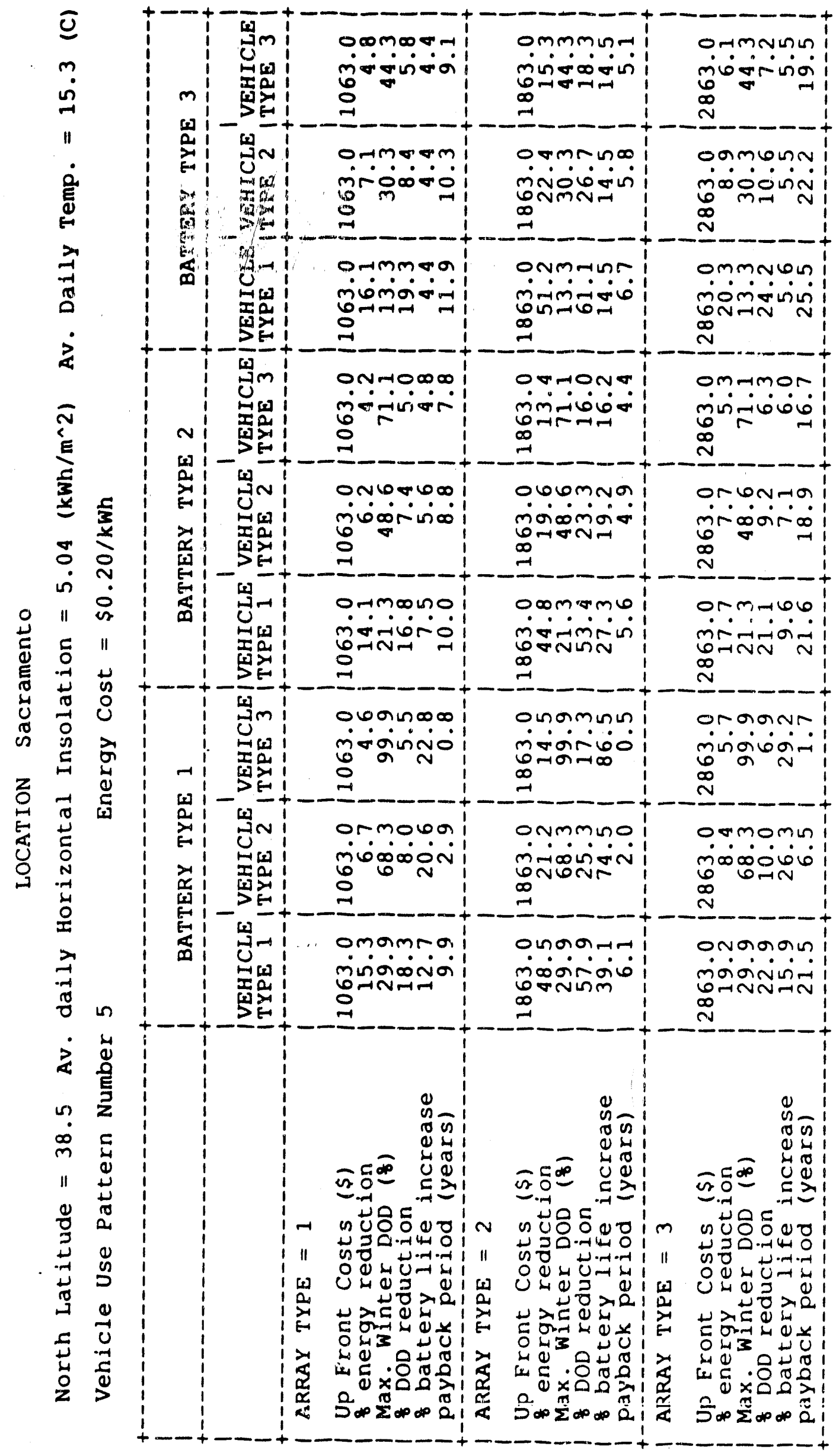




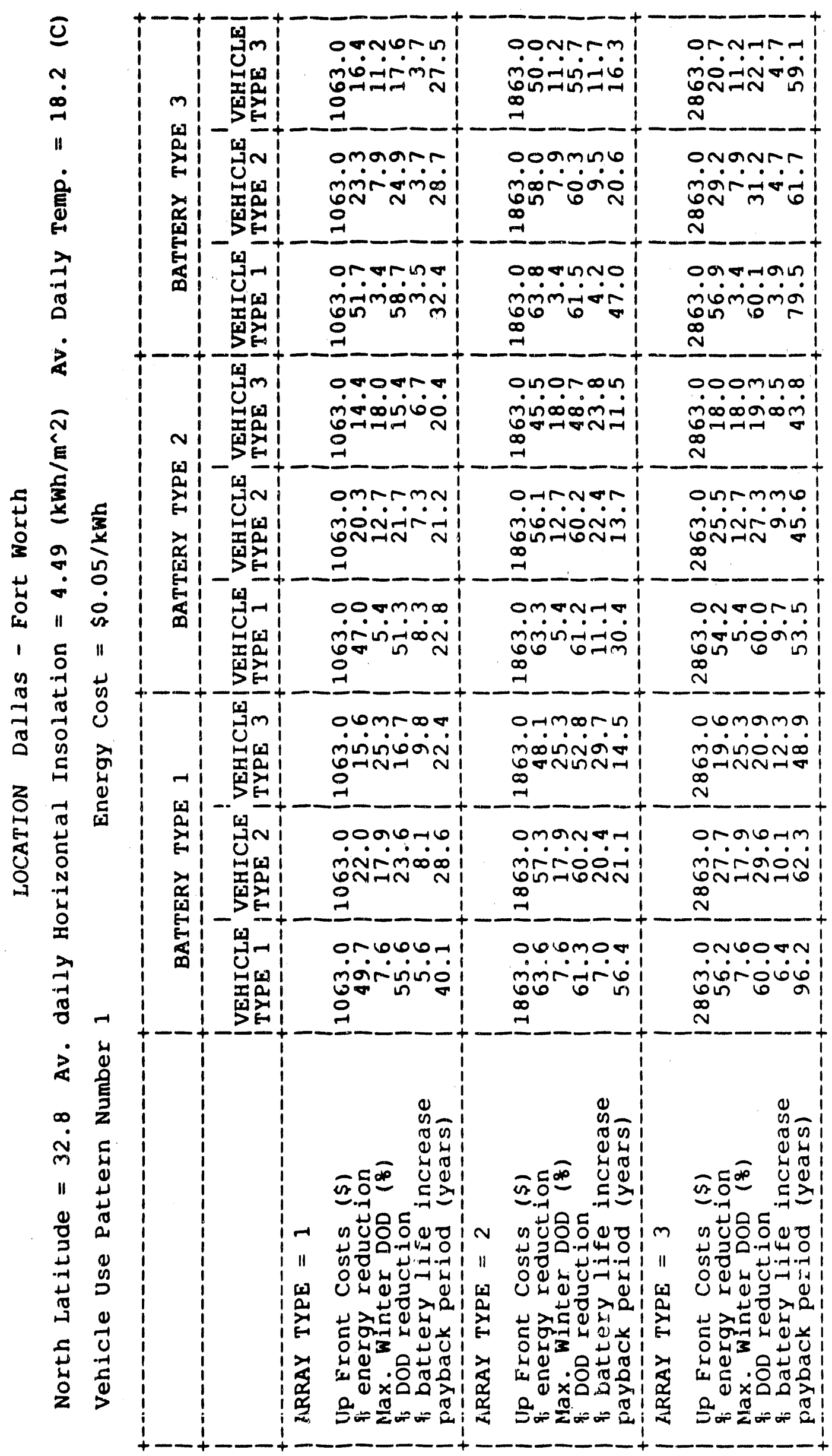




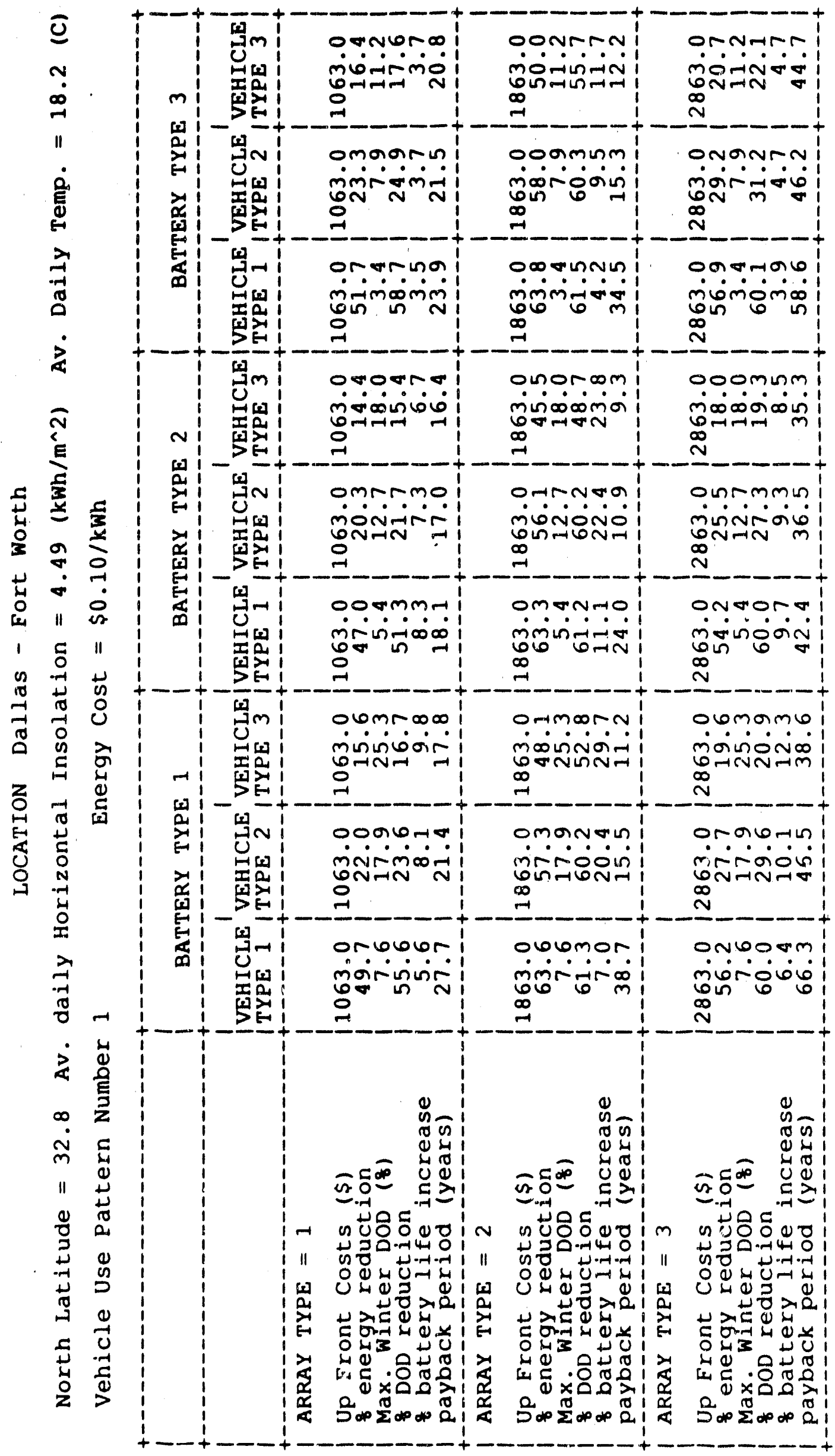




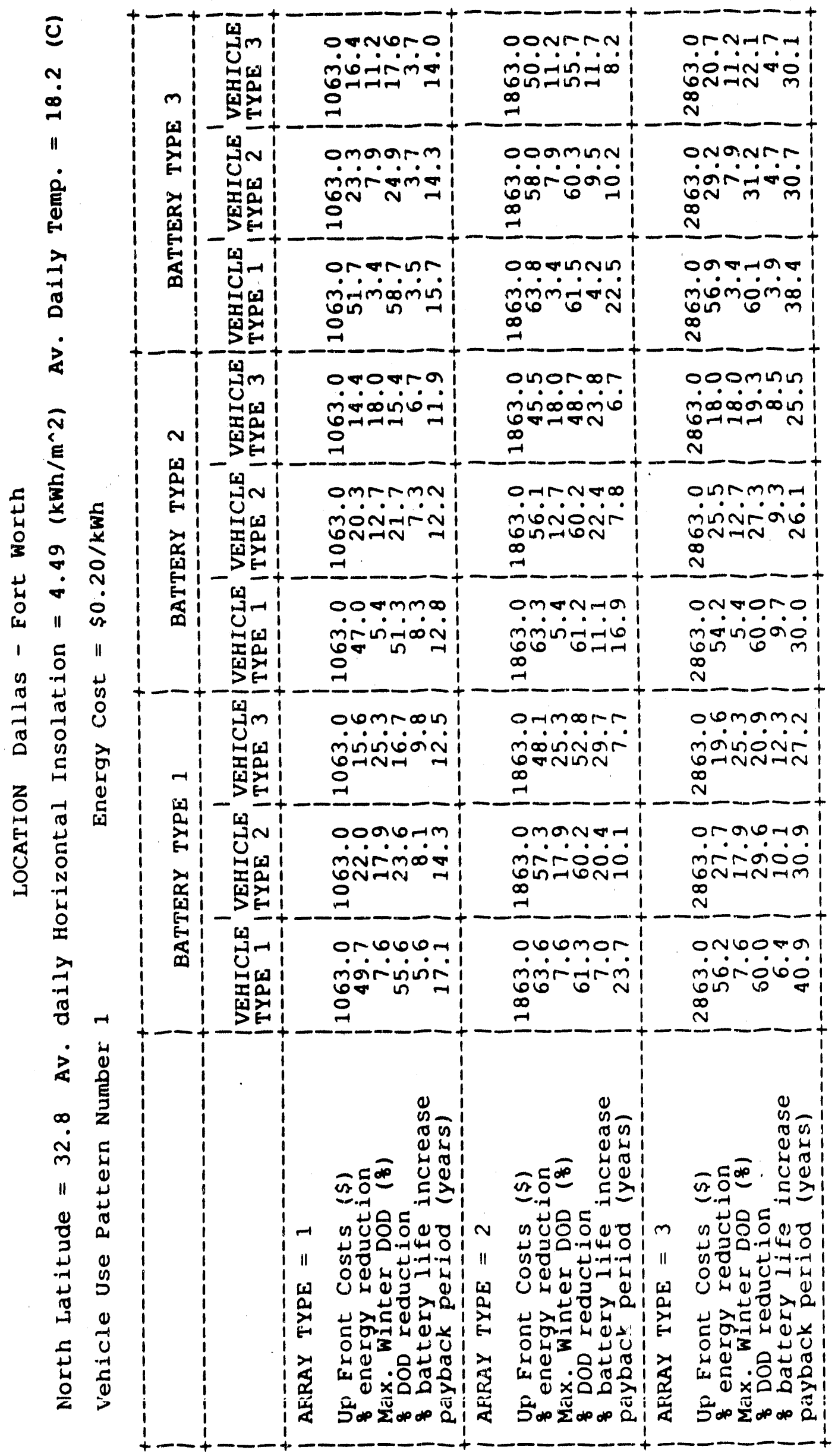




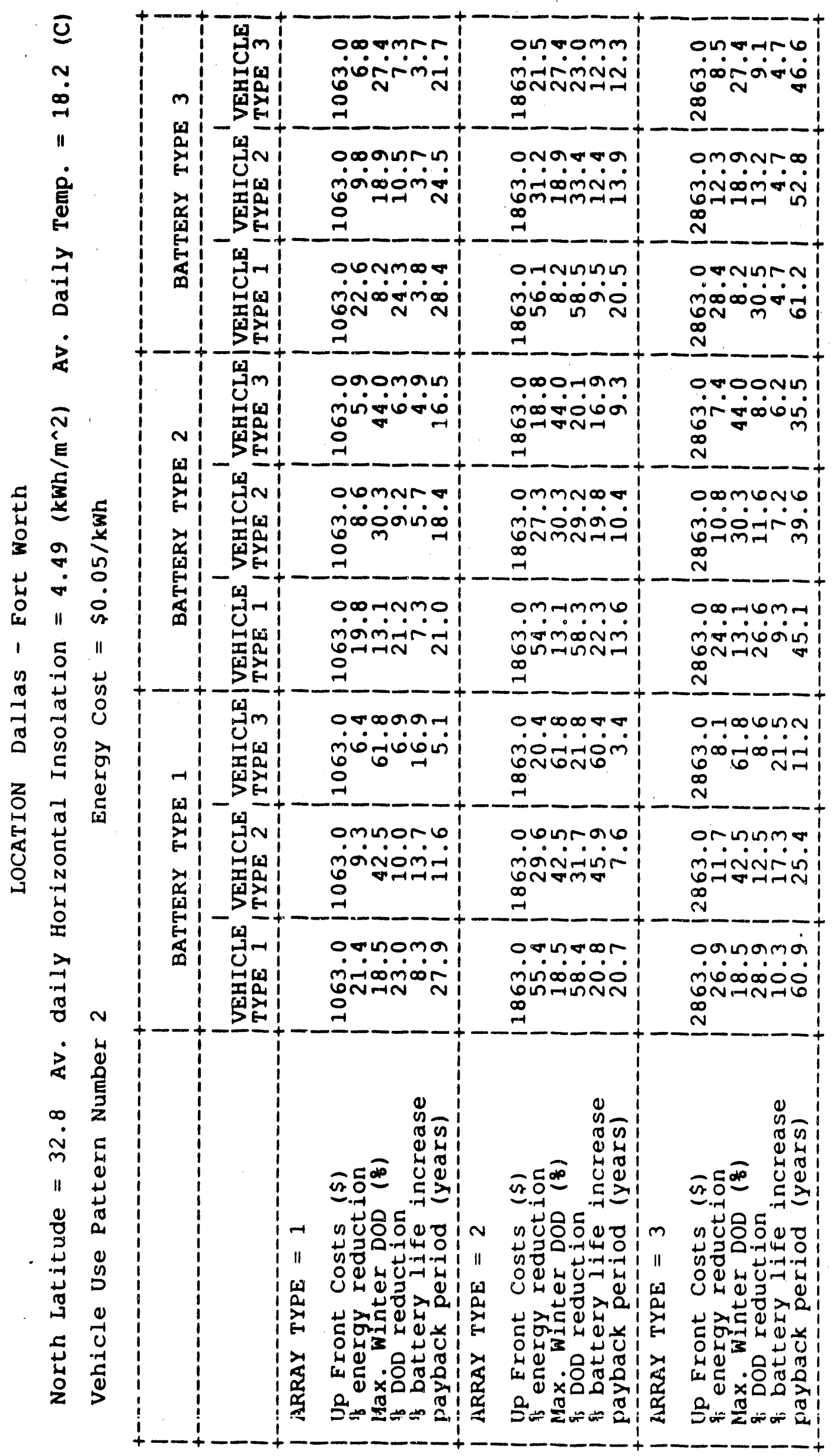




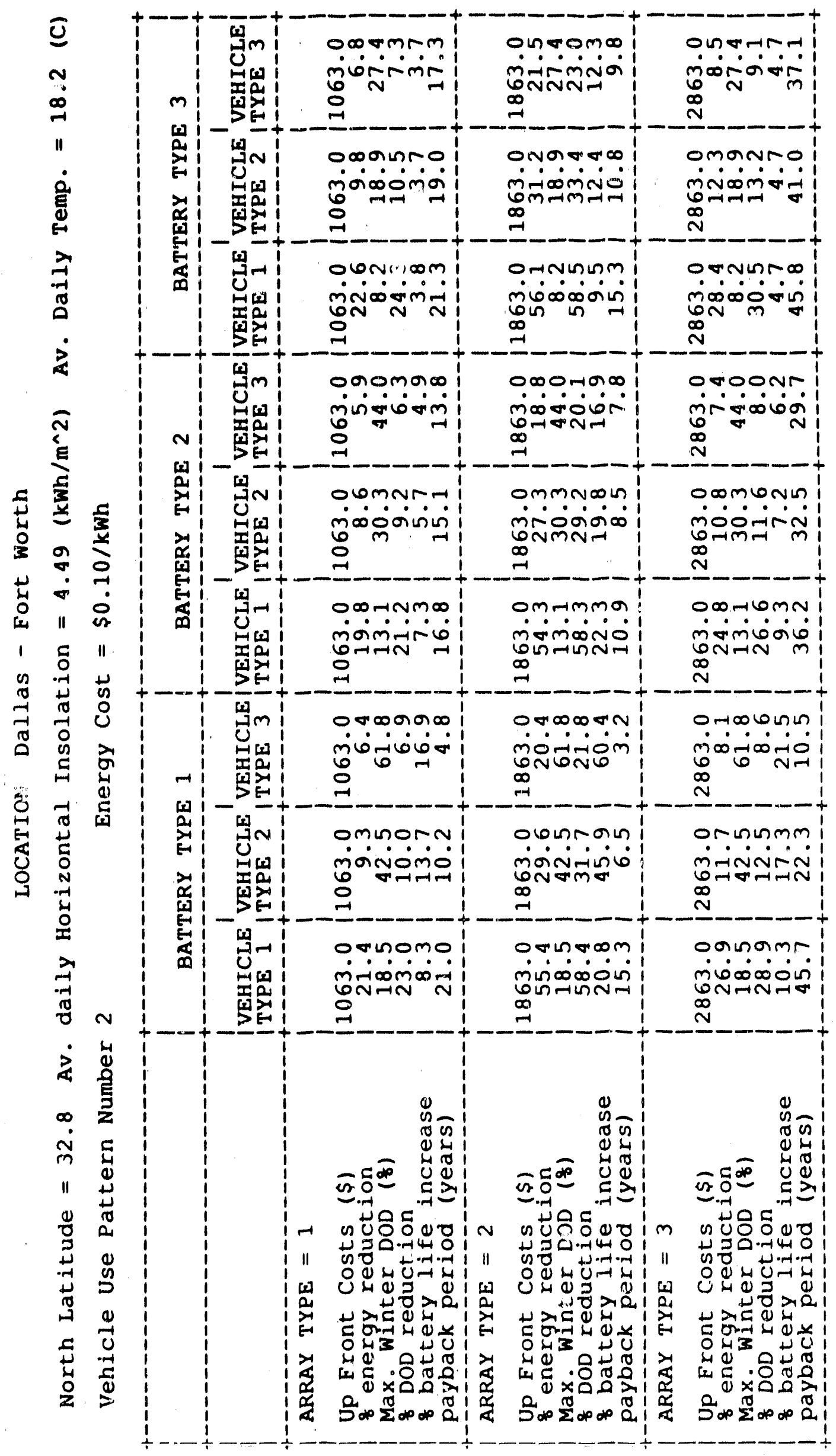




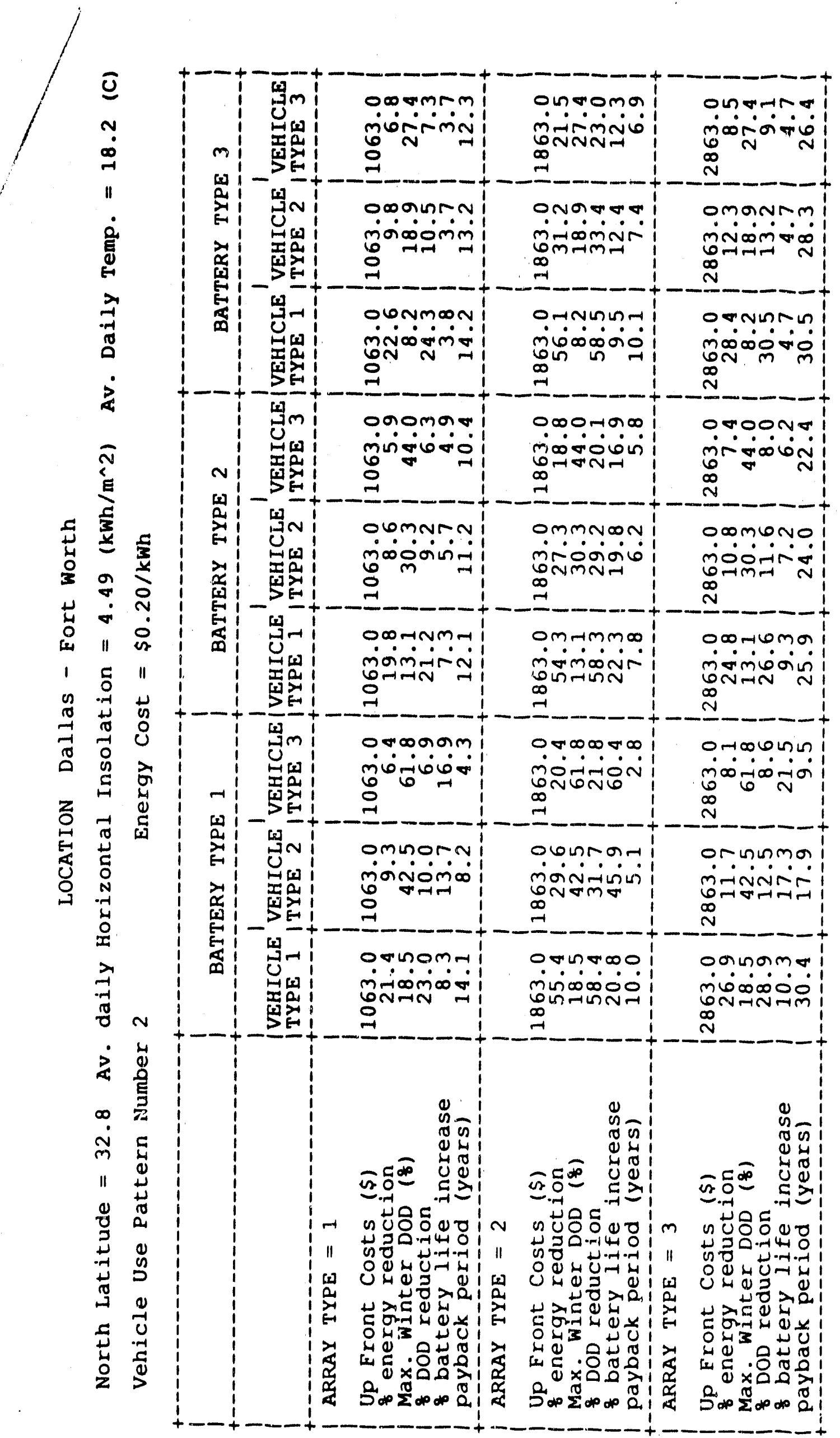




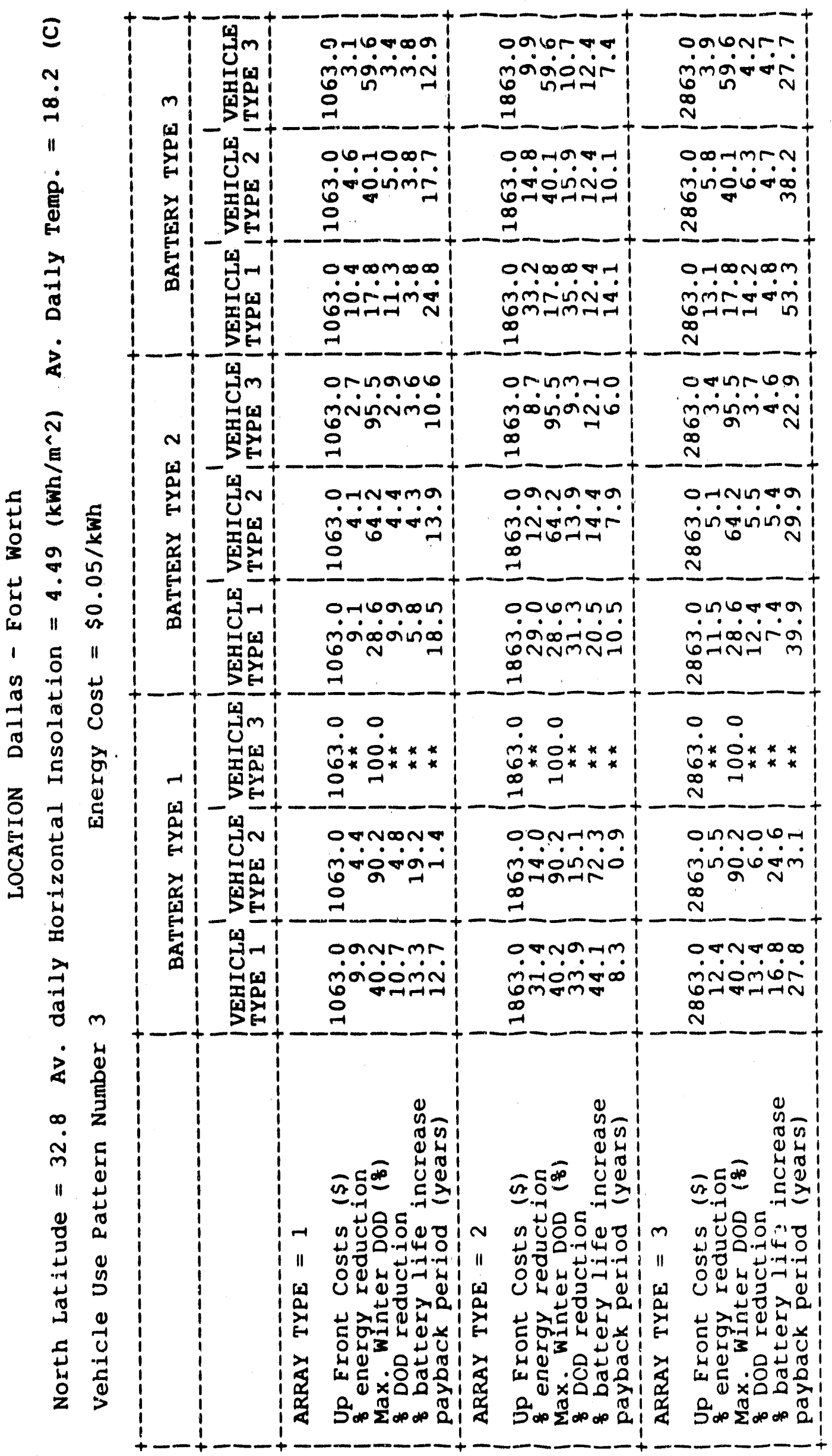




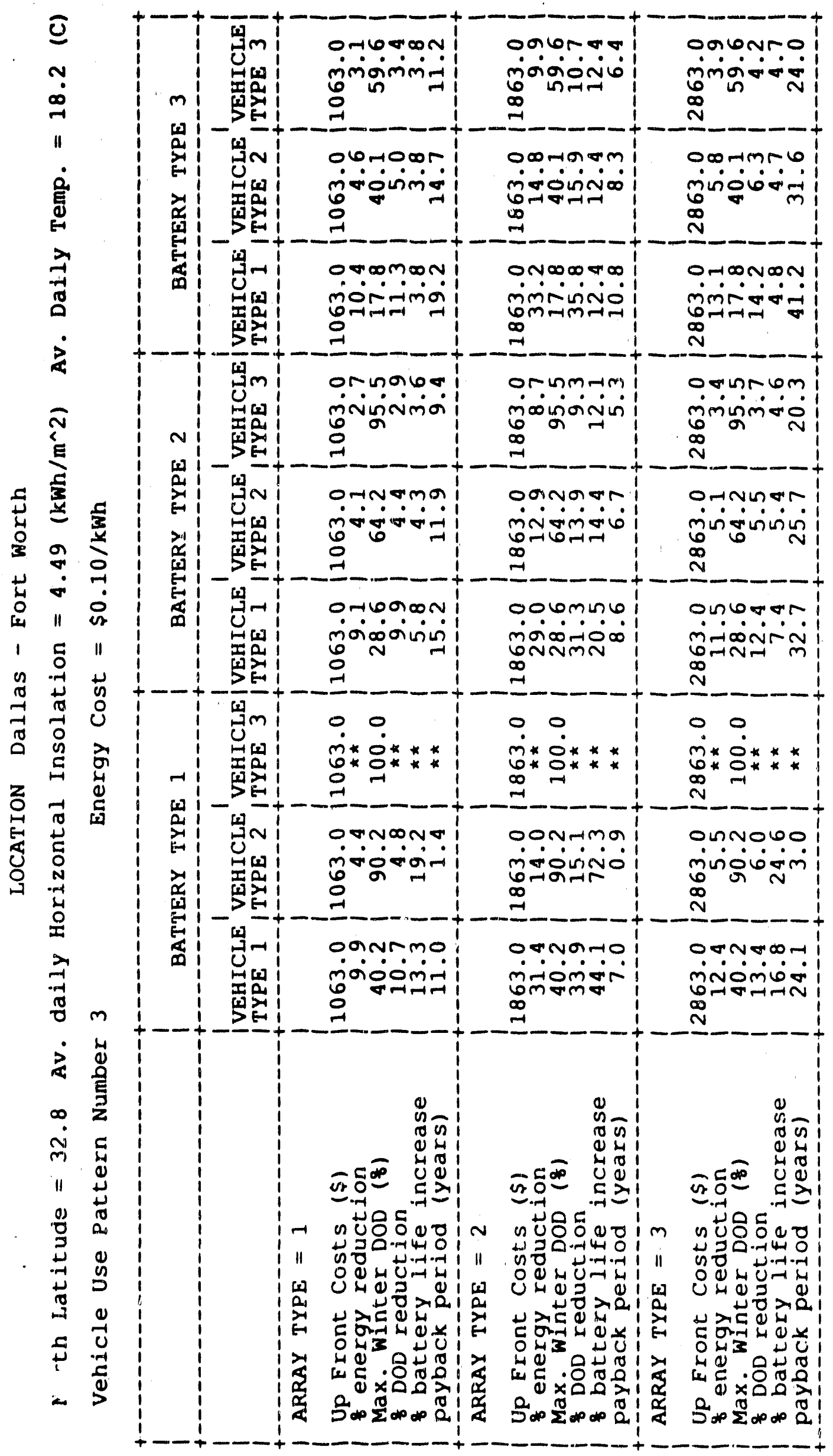




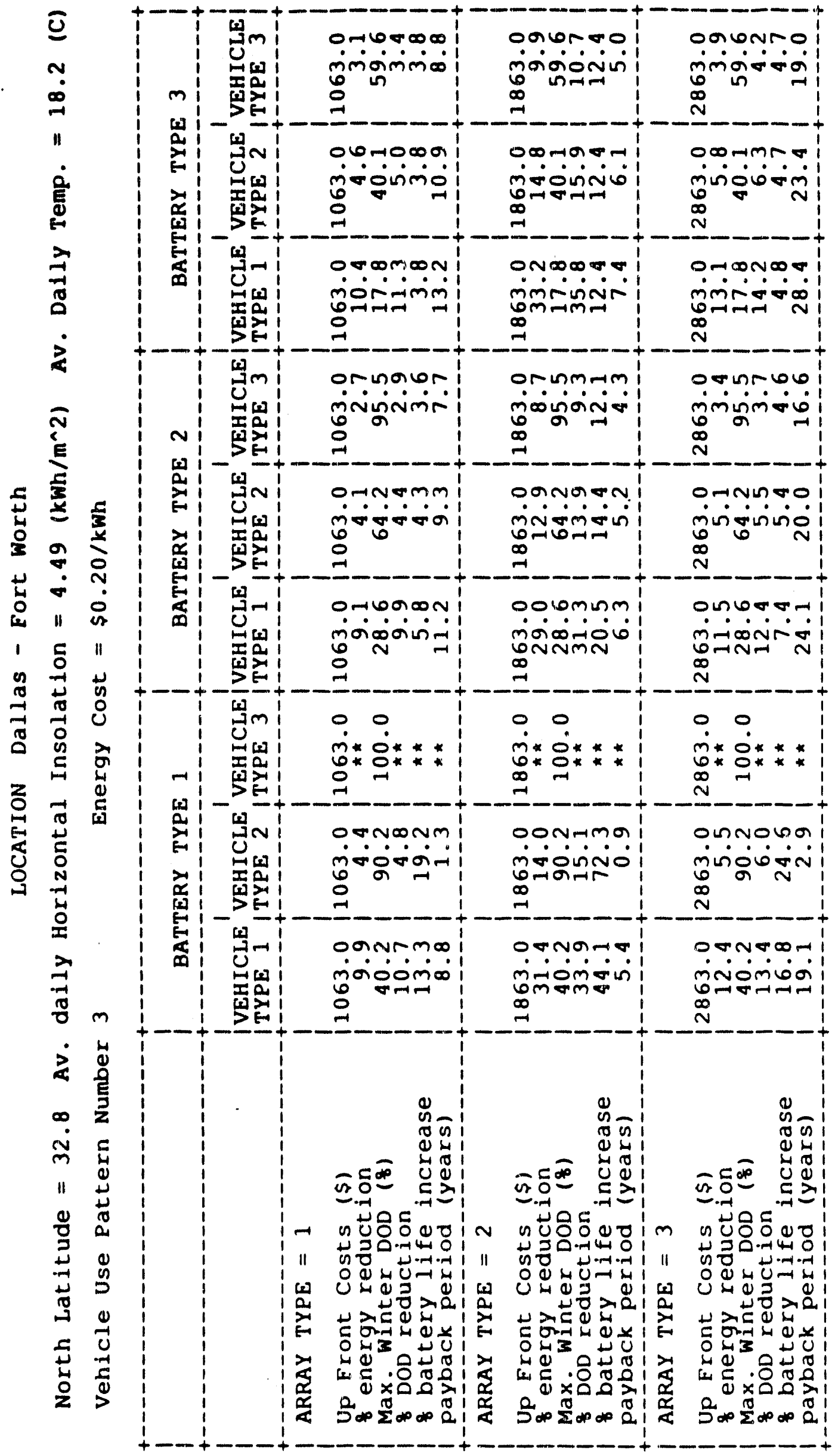




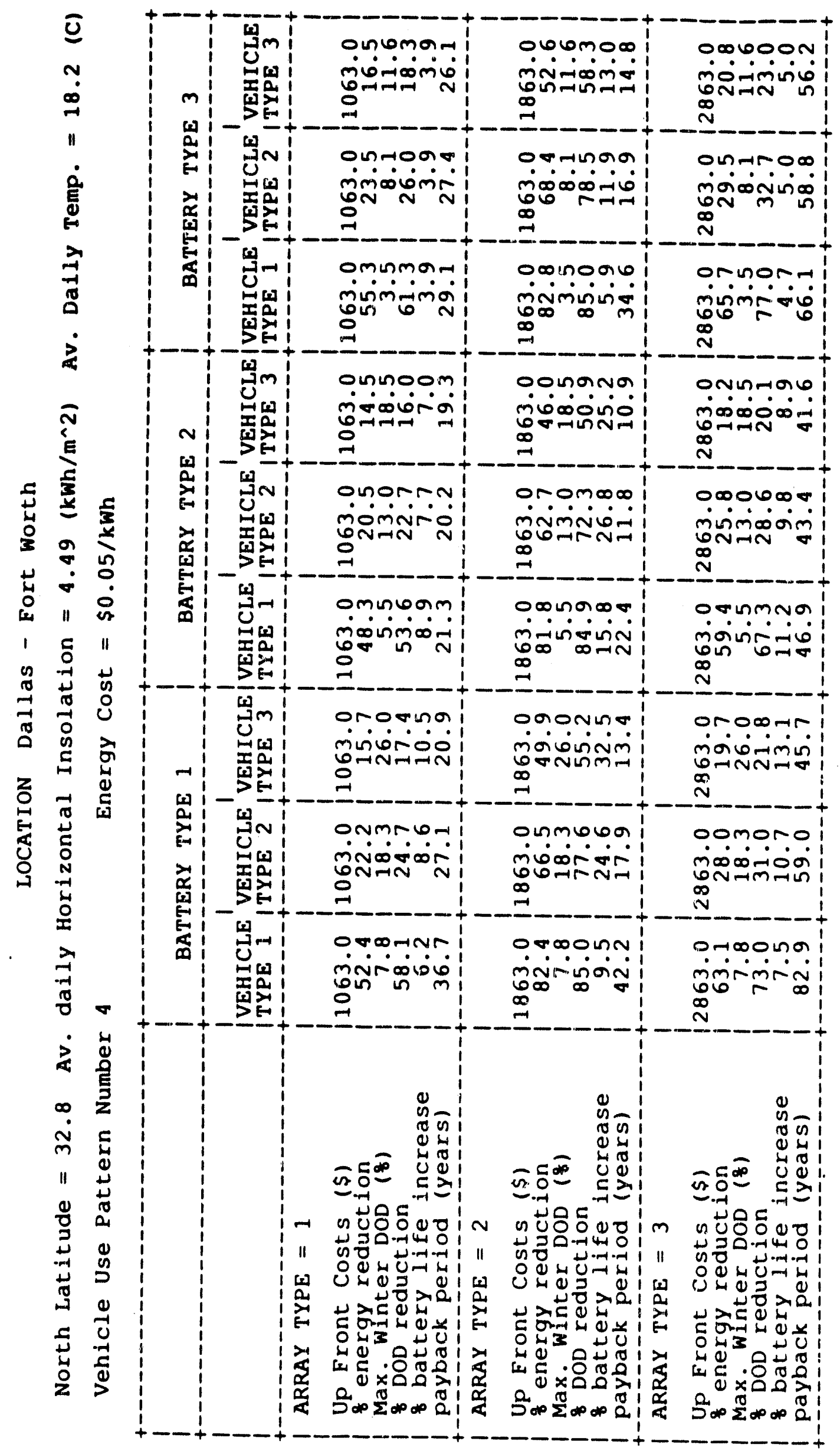




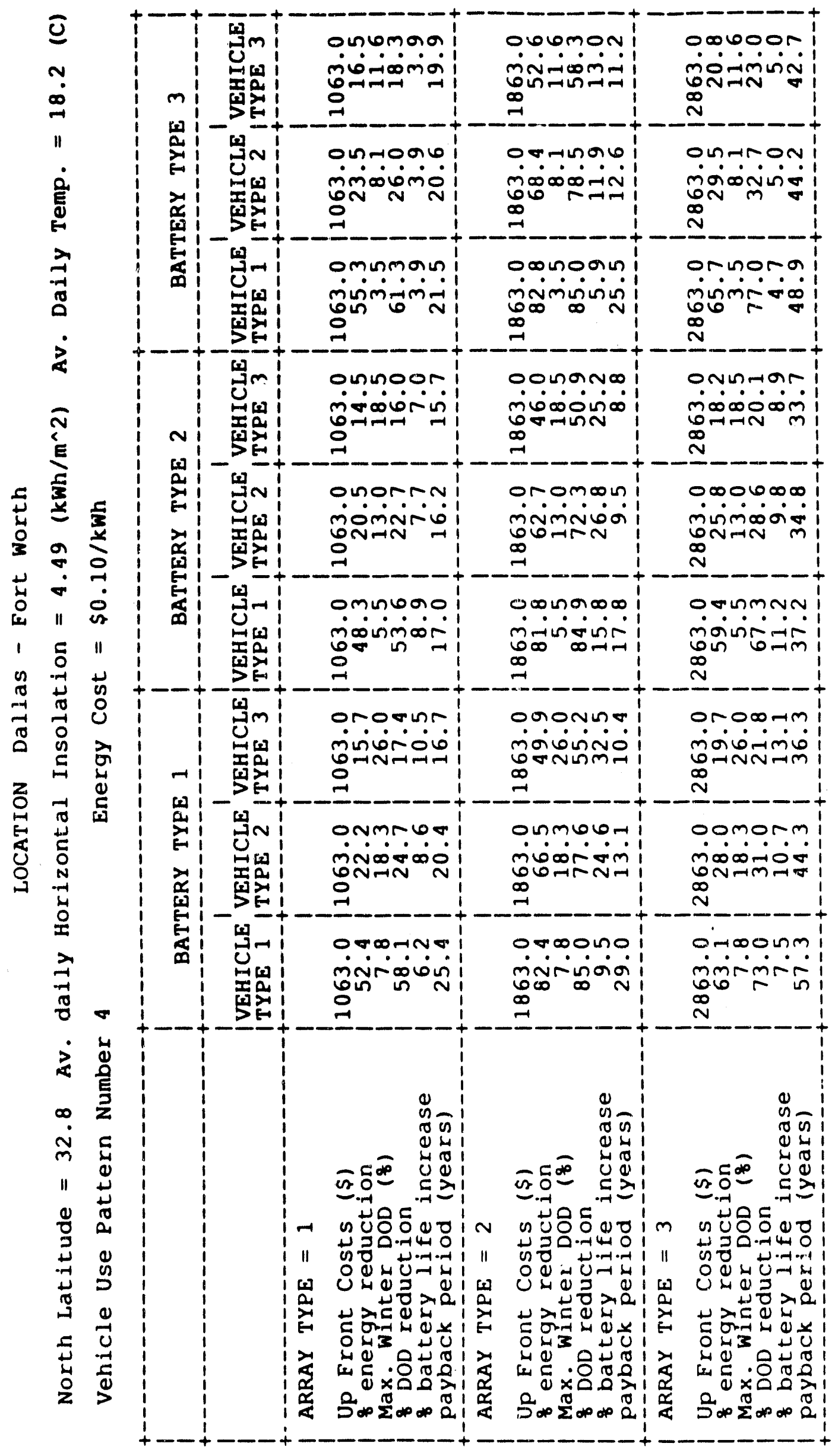




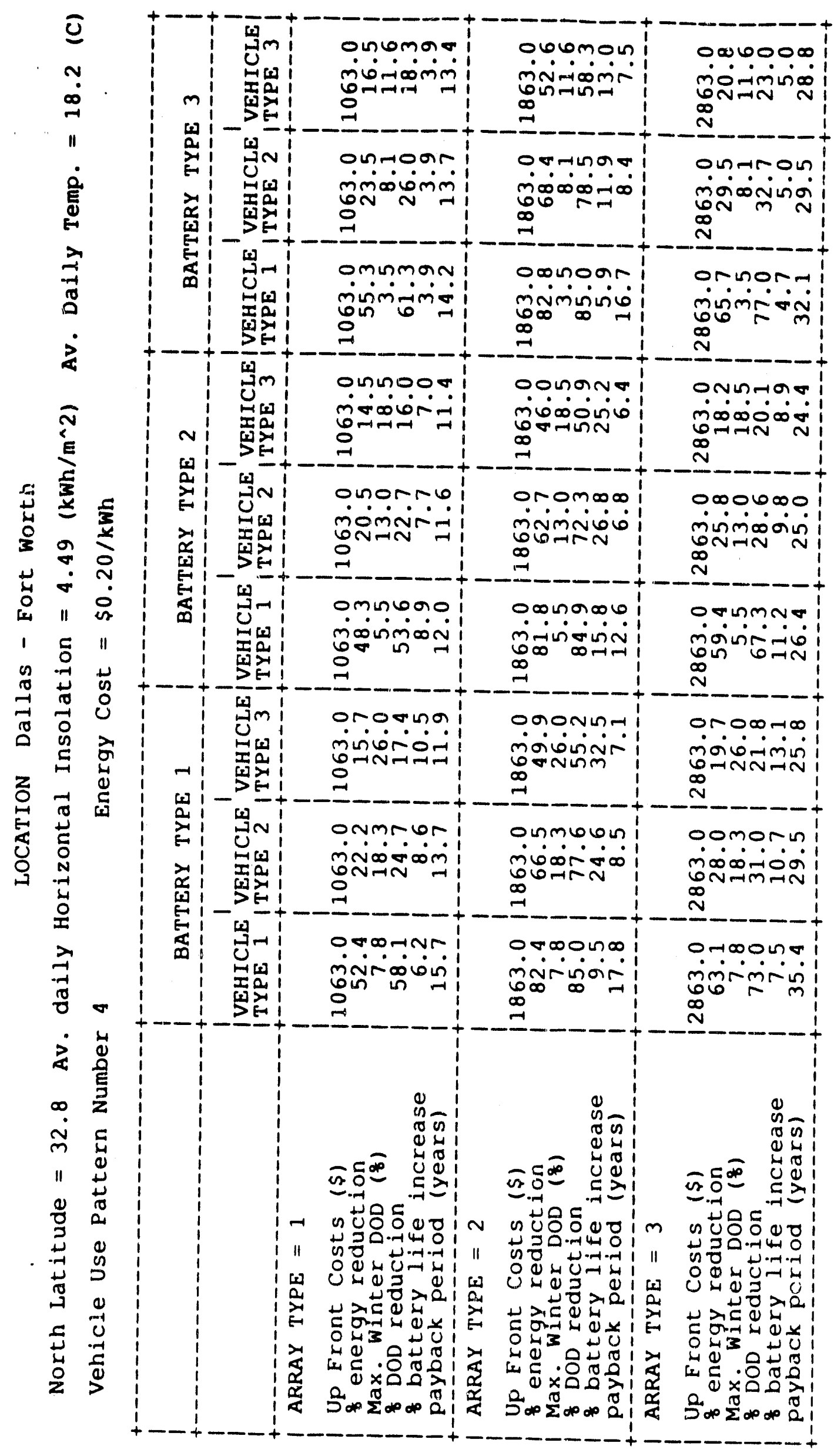

1
1
1
1 


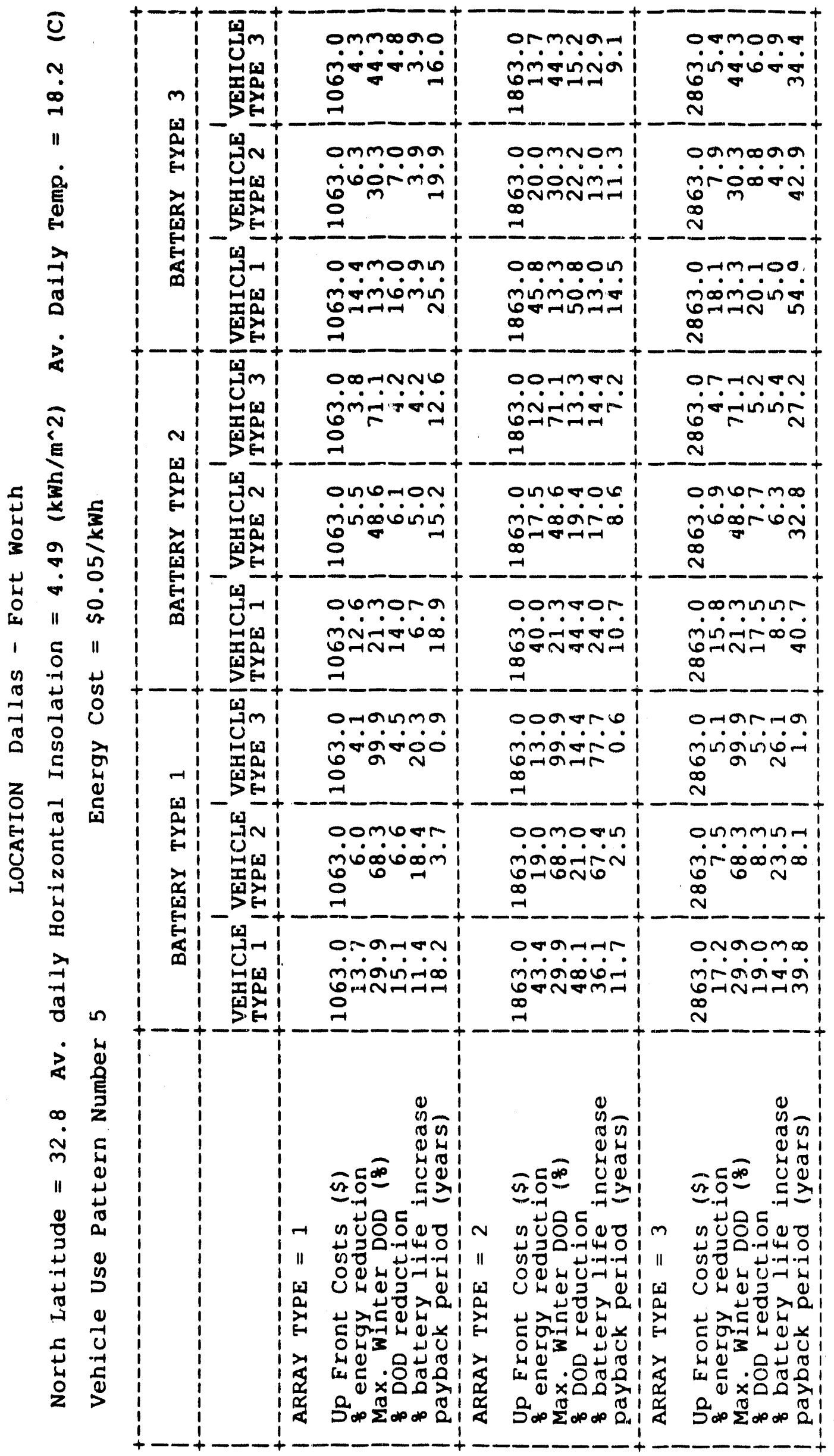




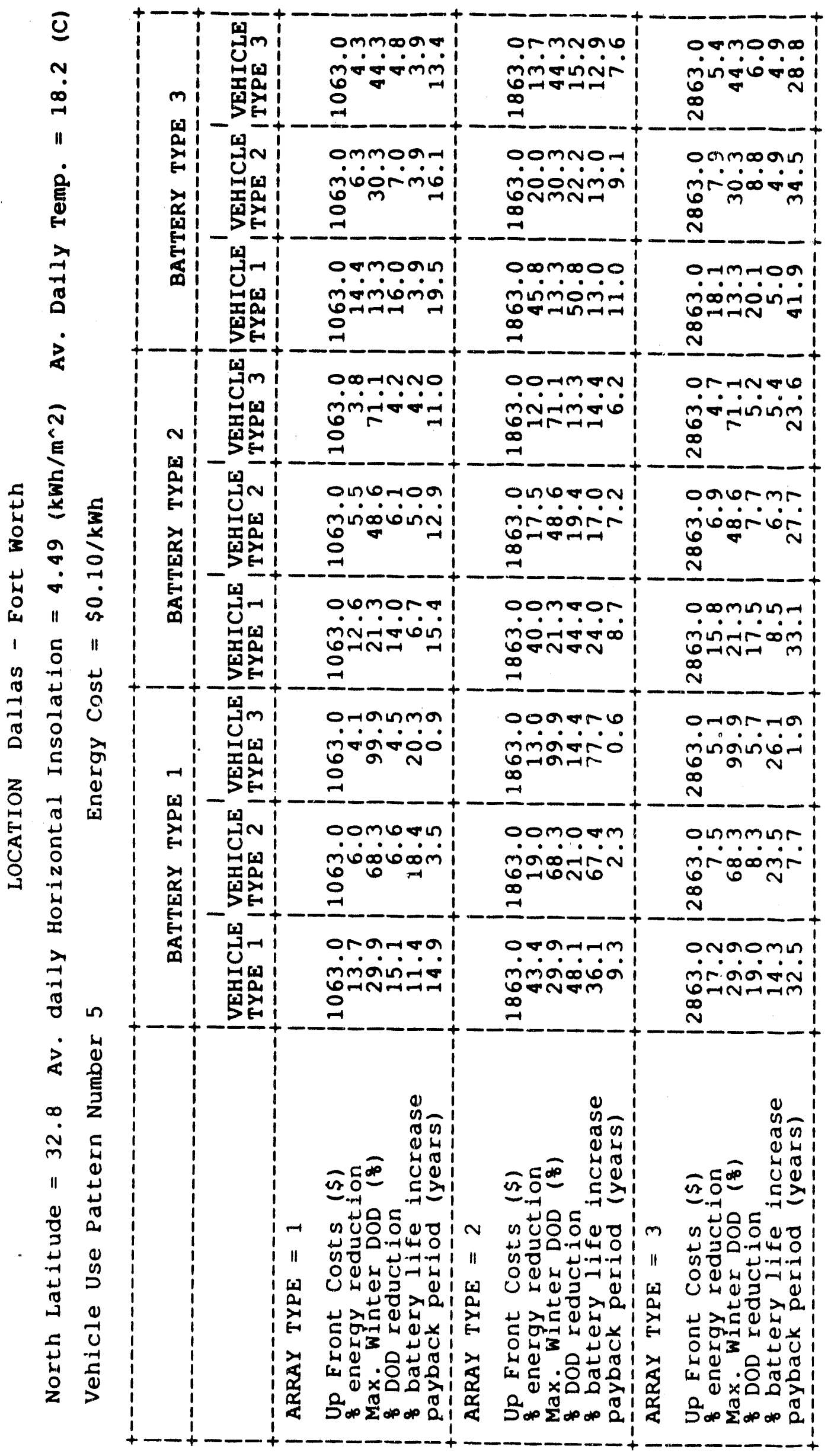




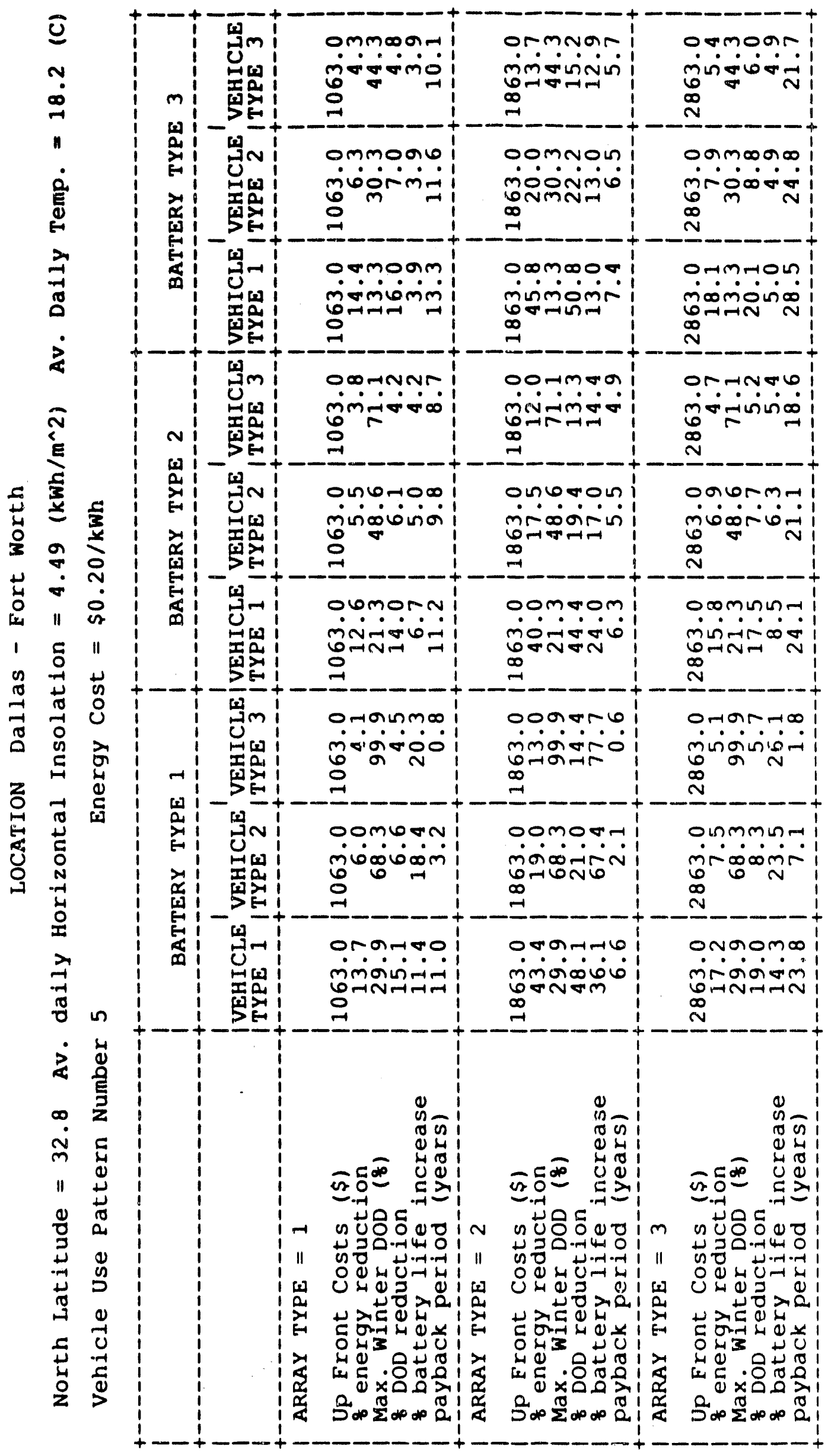




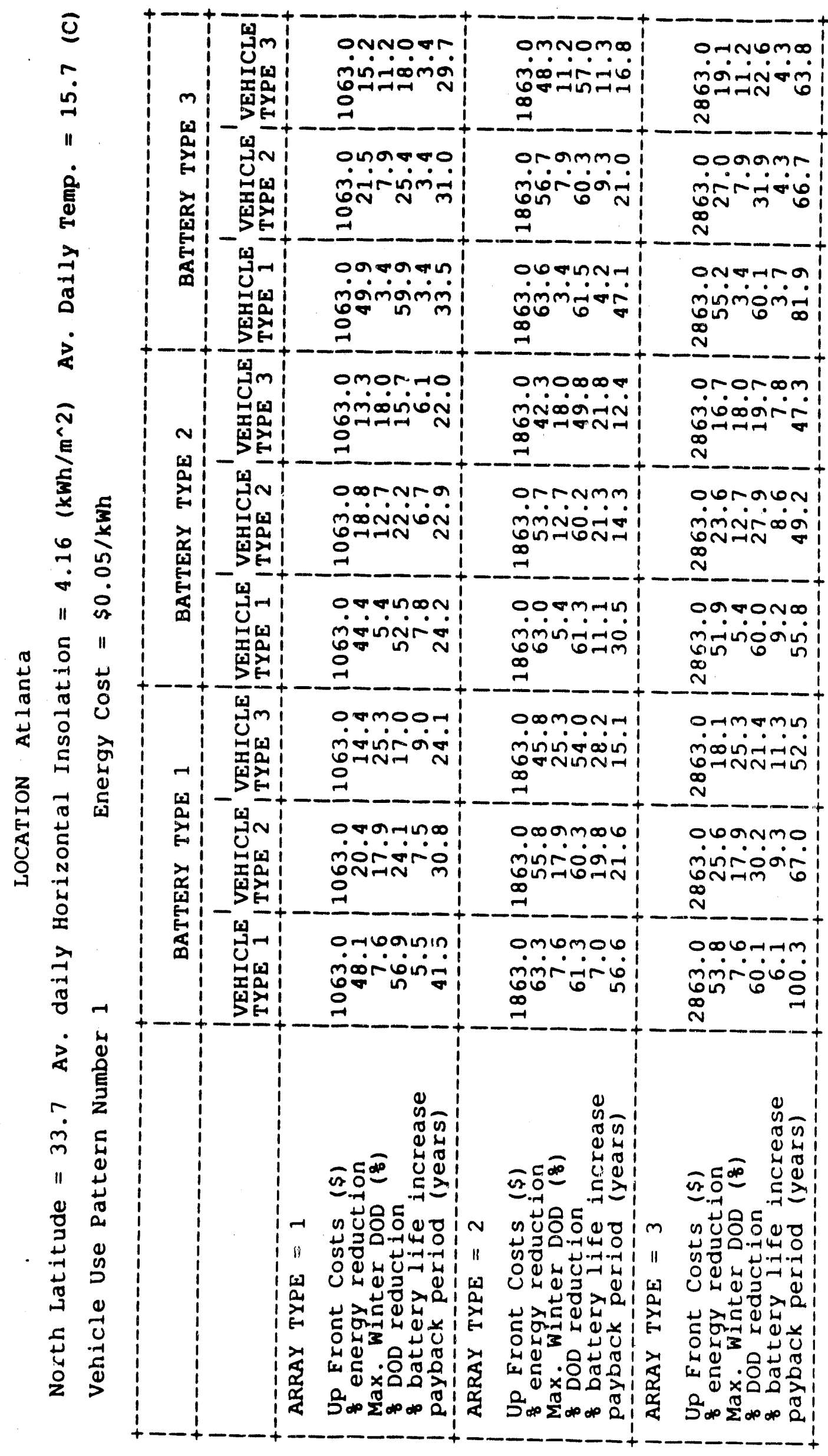




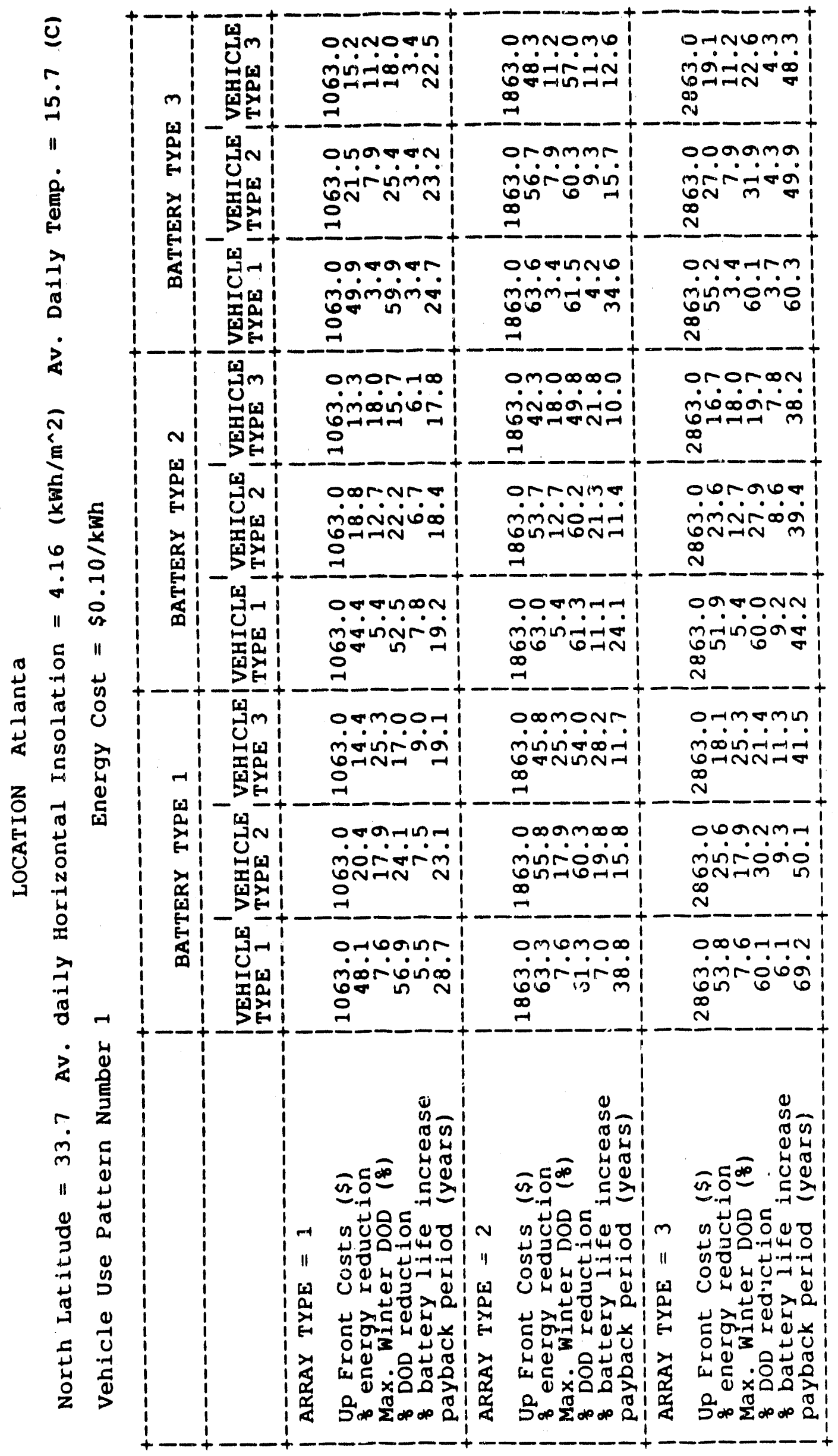




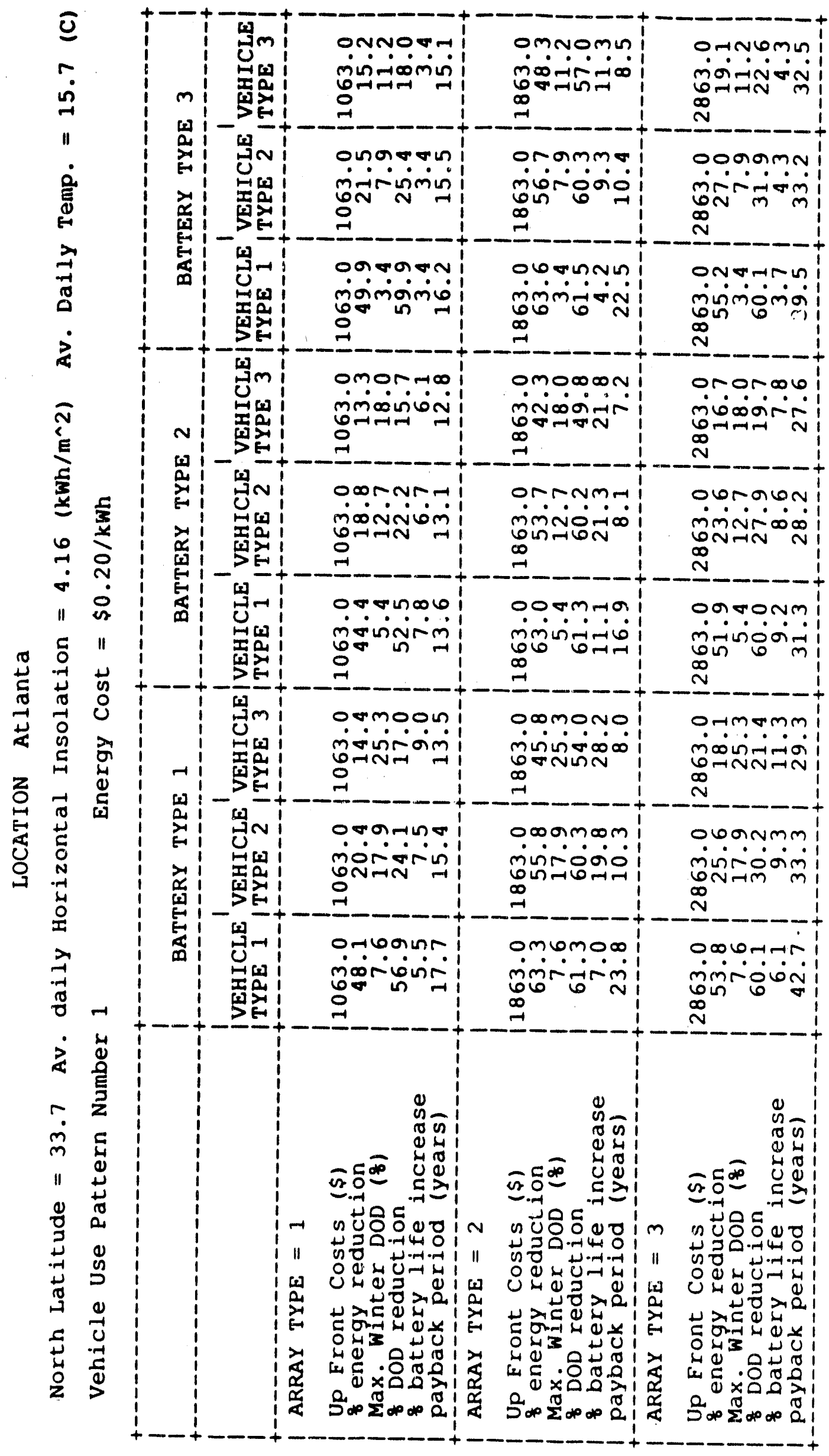




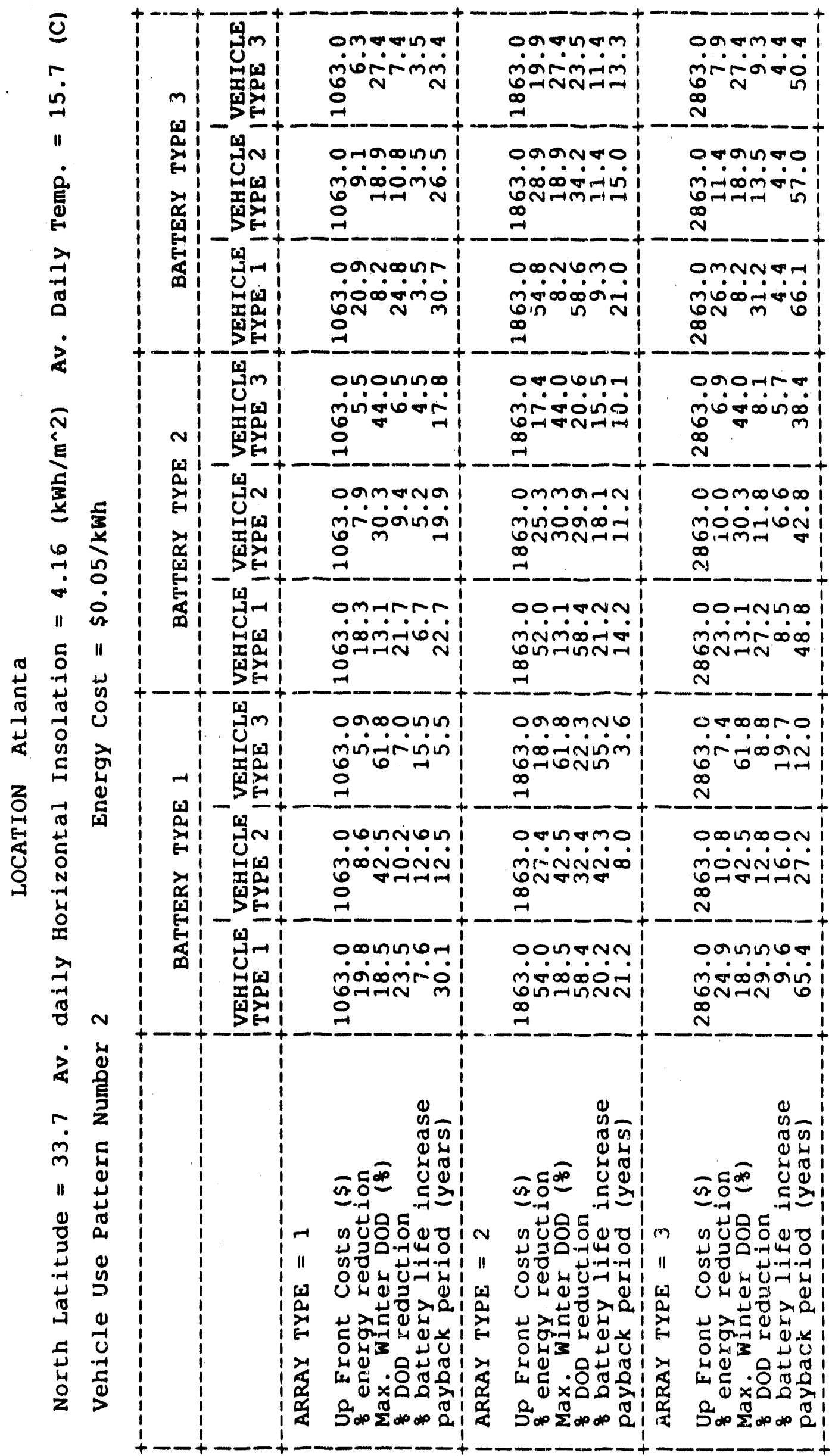




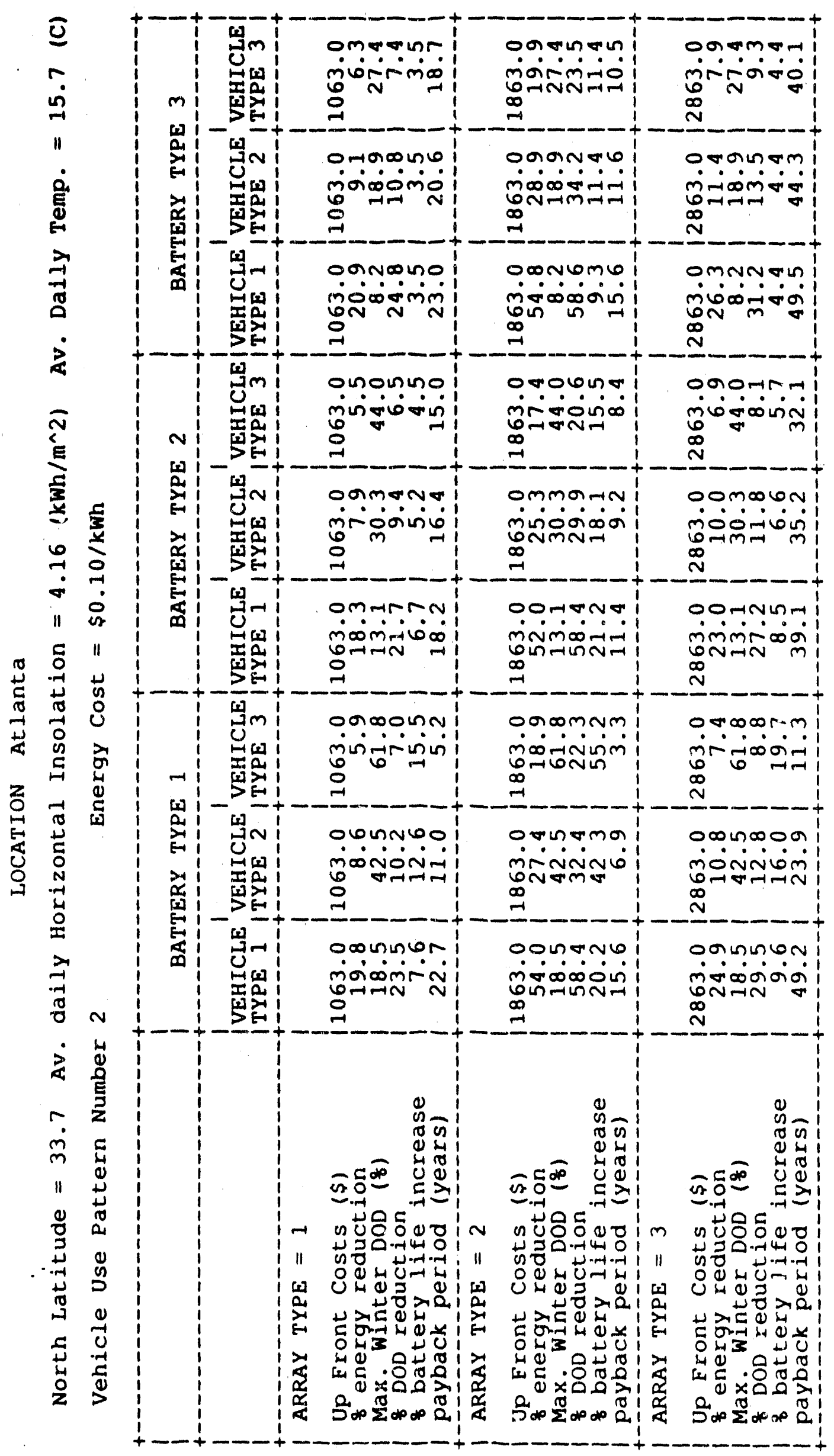




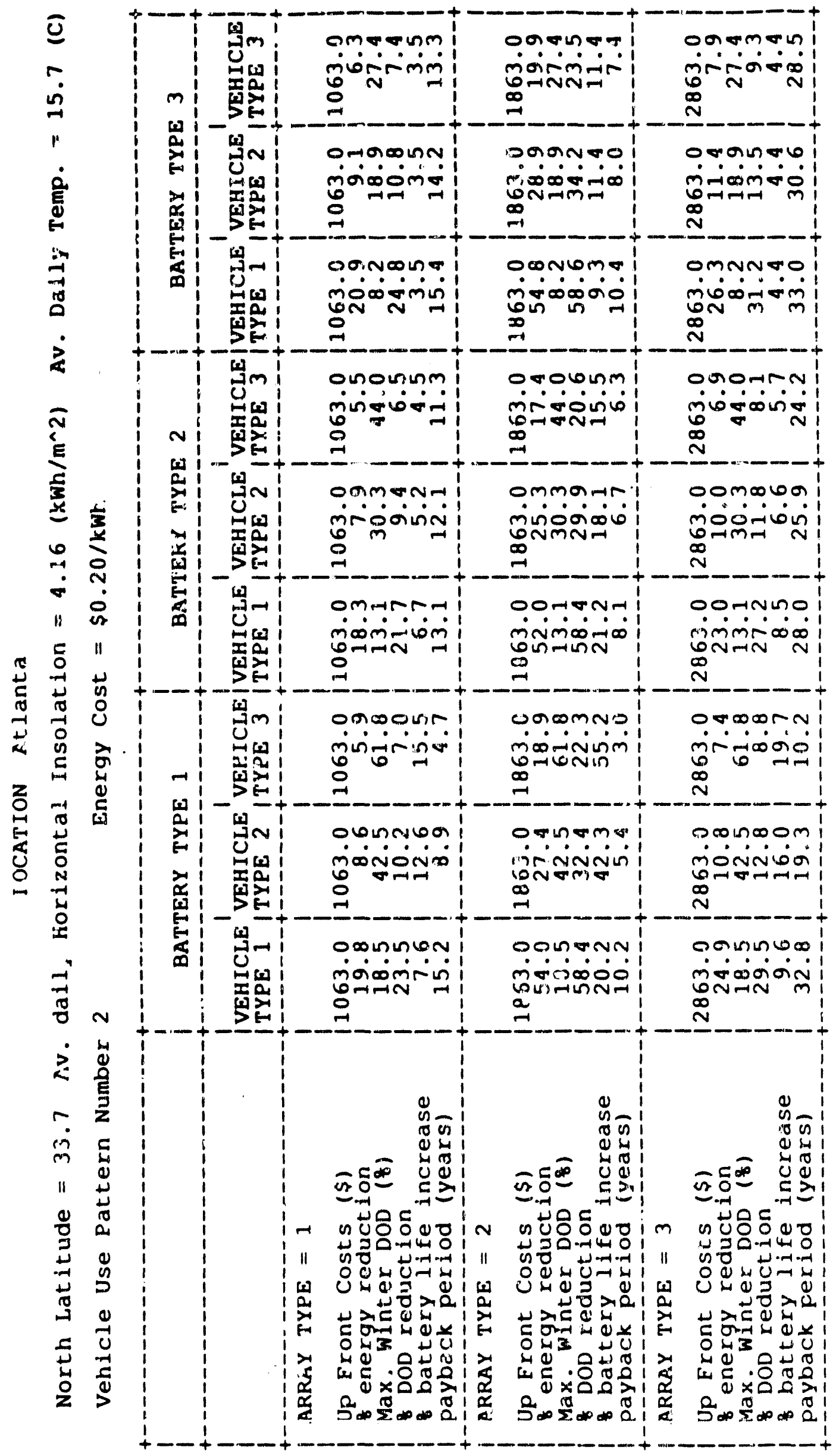




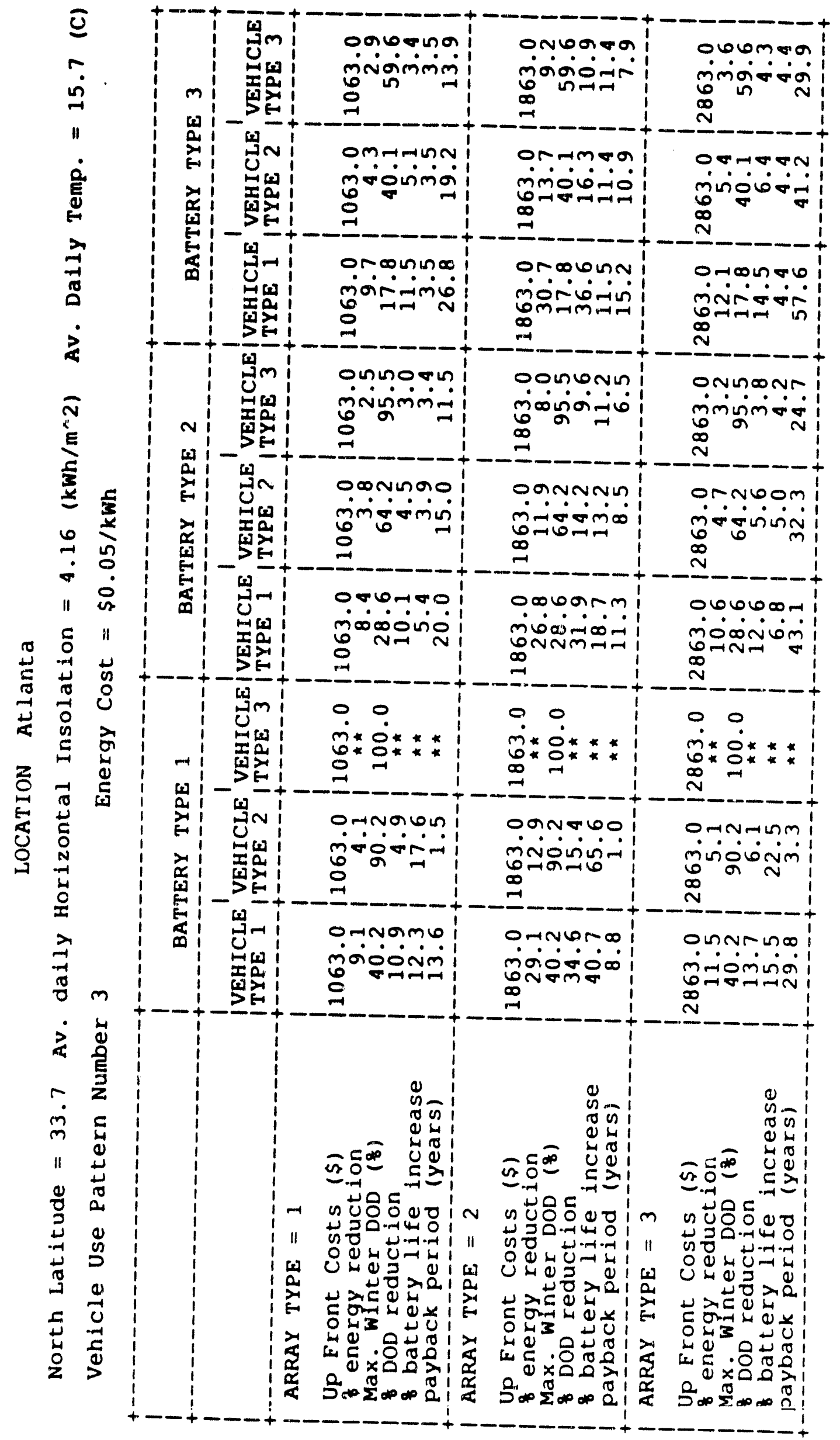




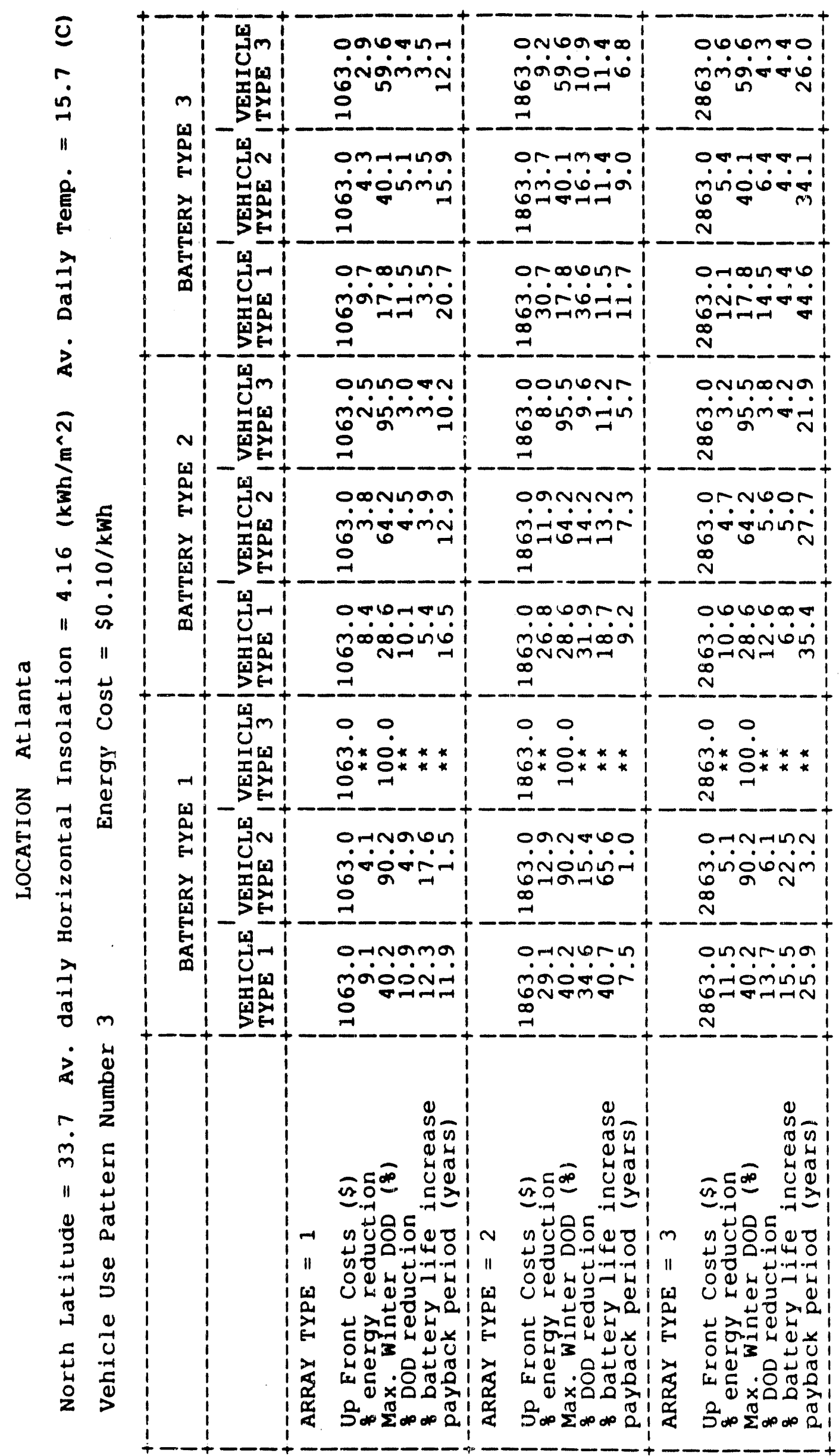




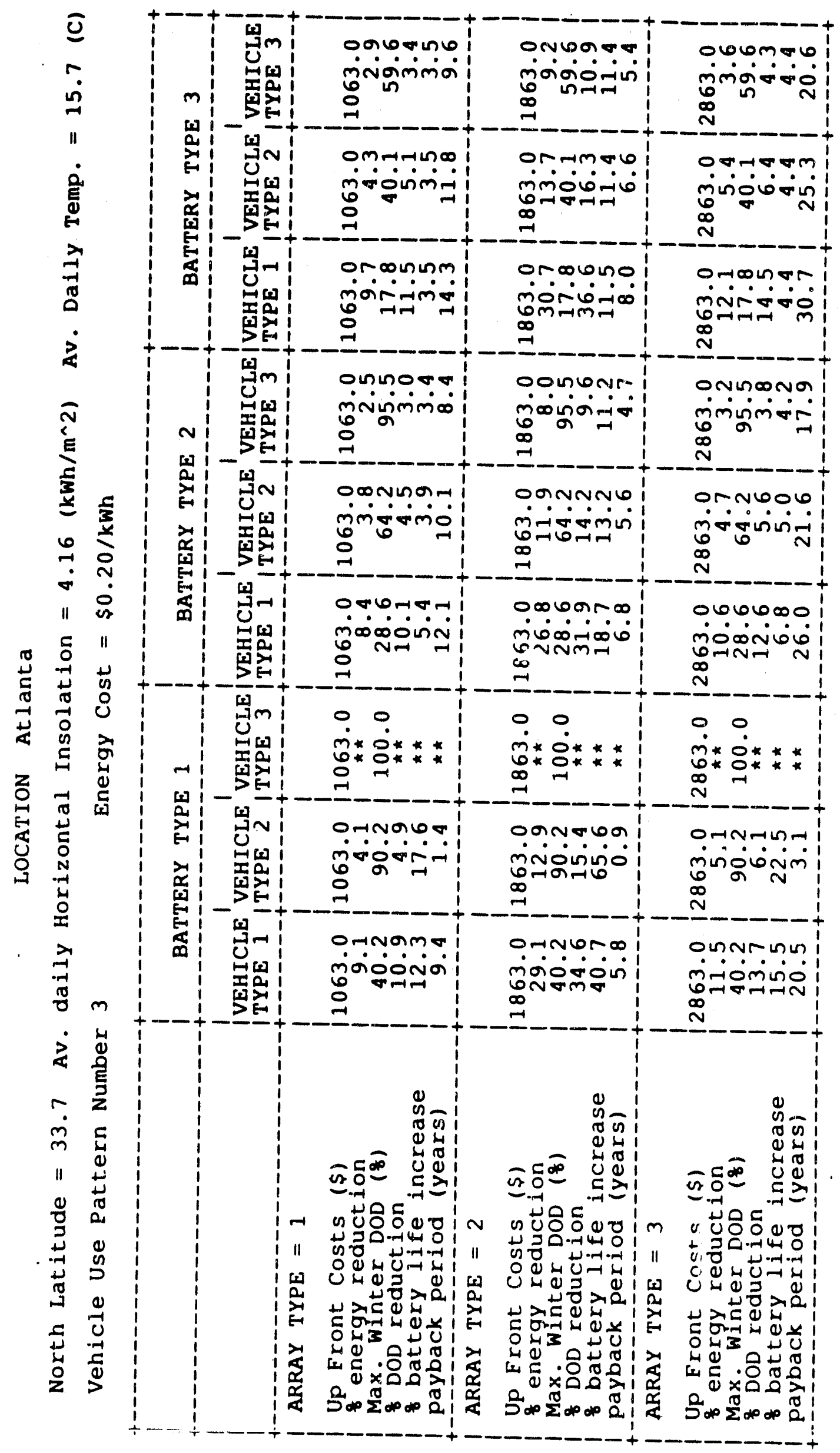




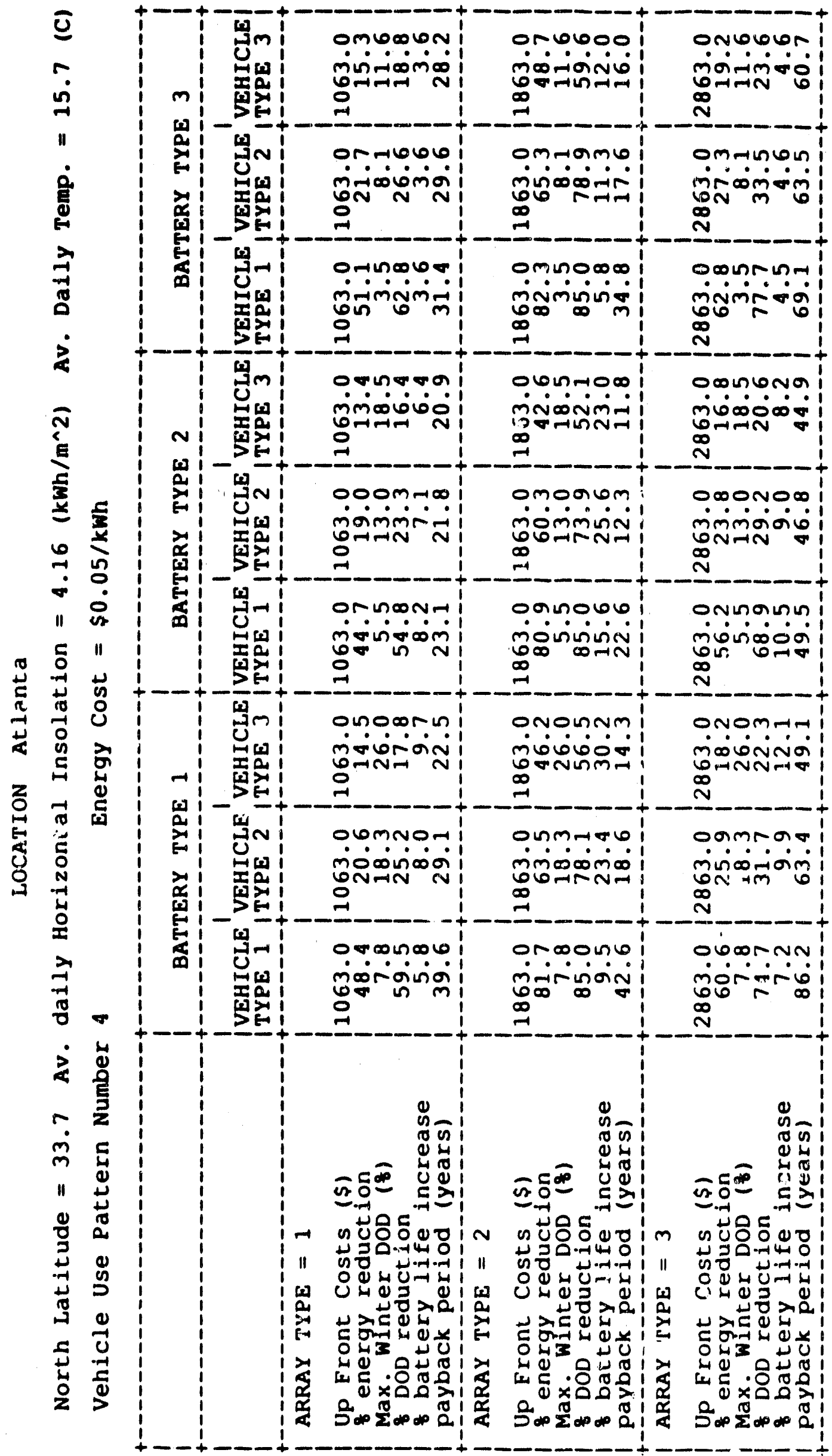




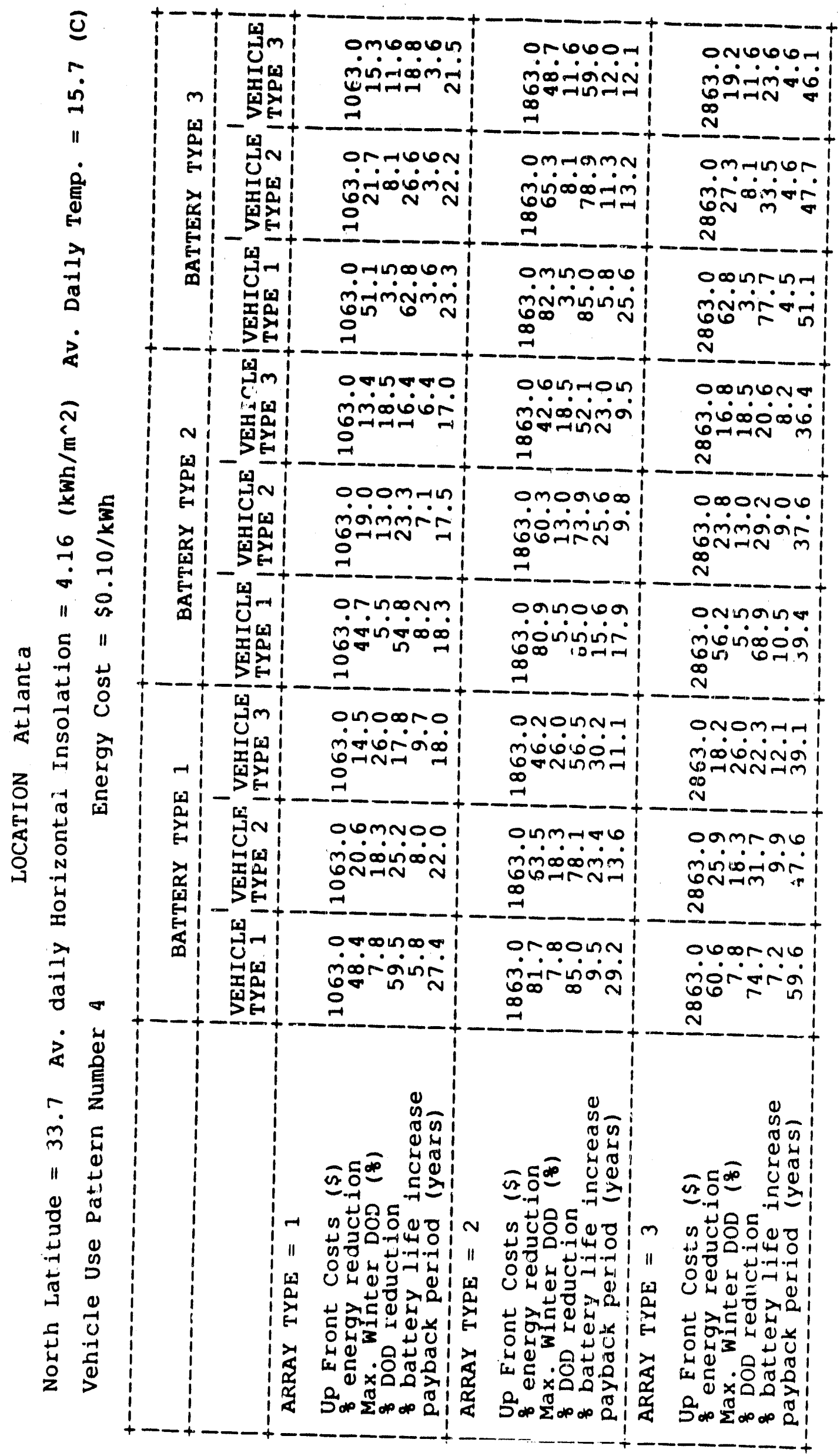




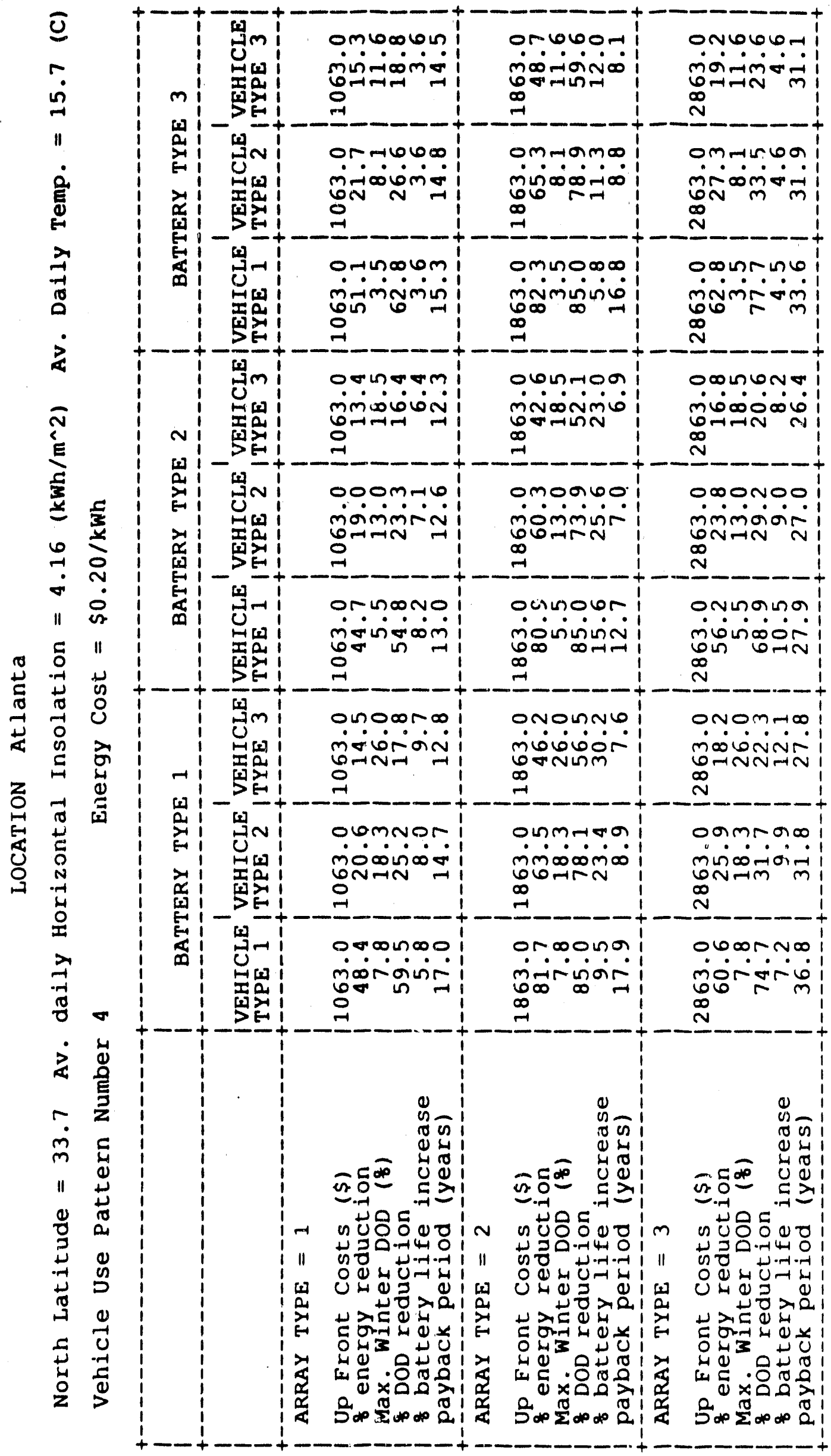




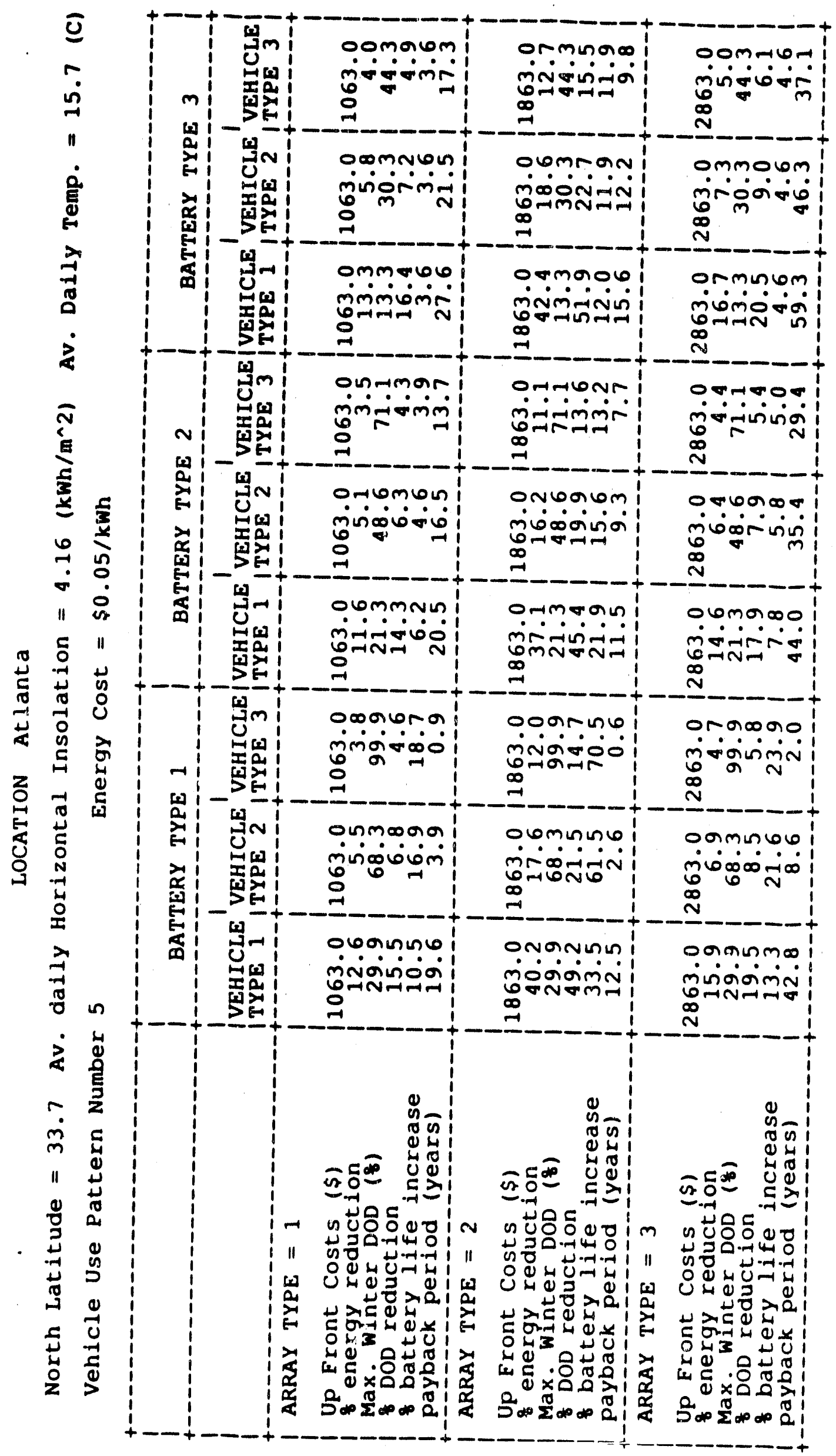




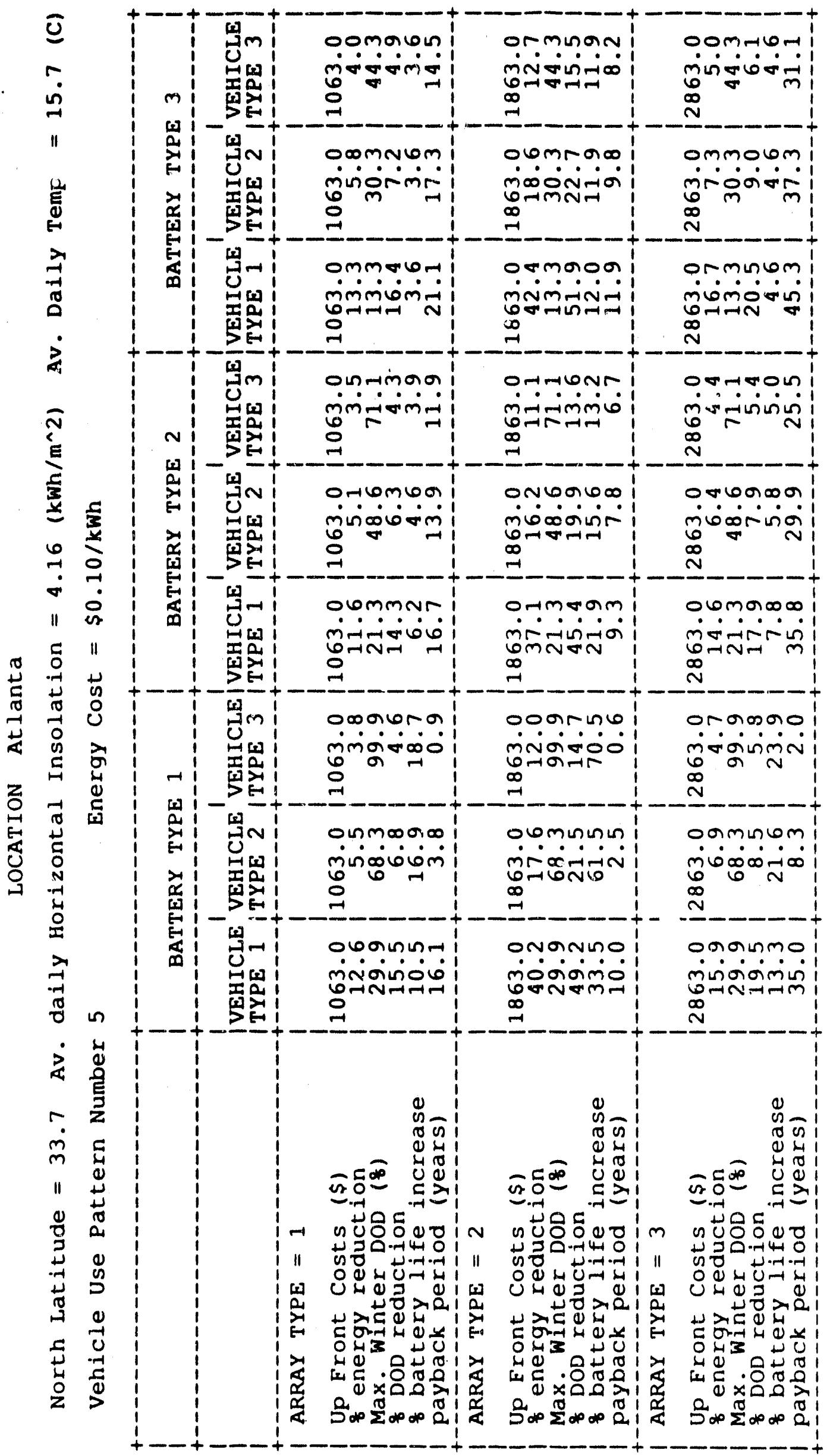




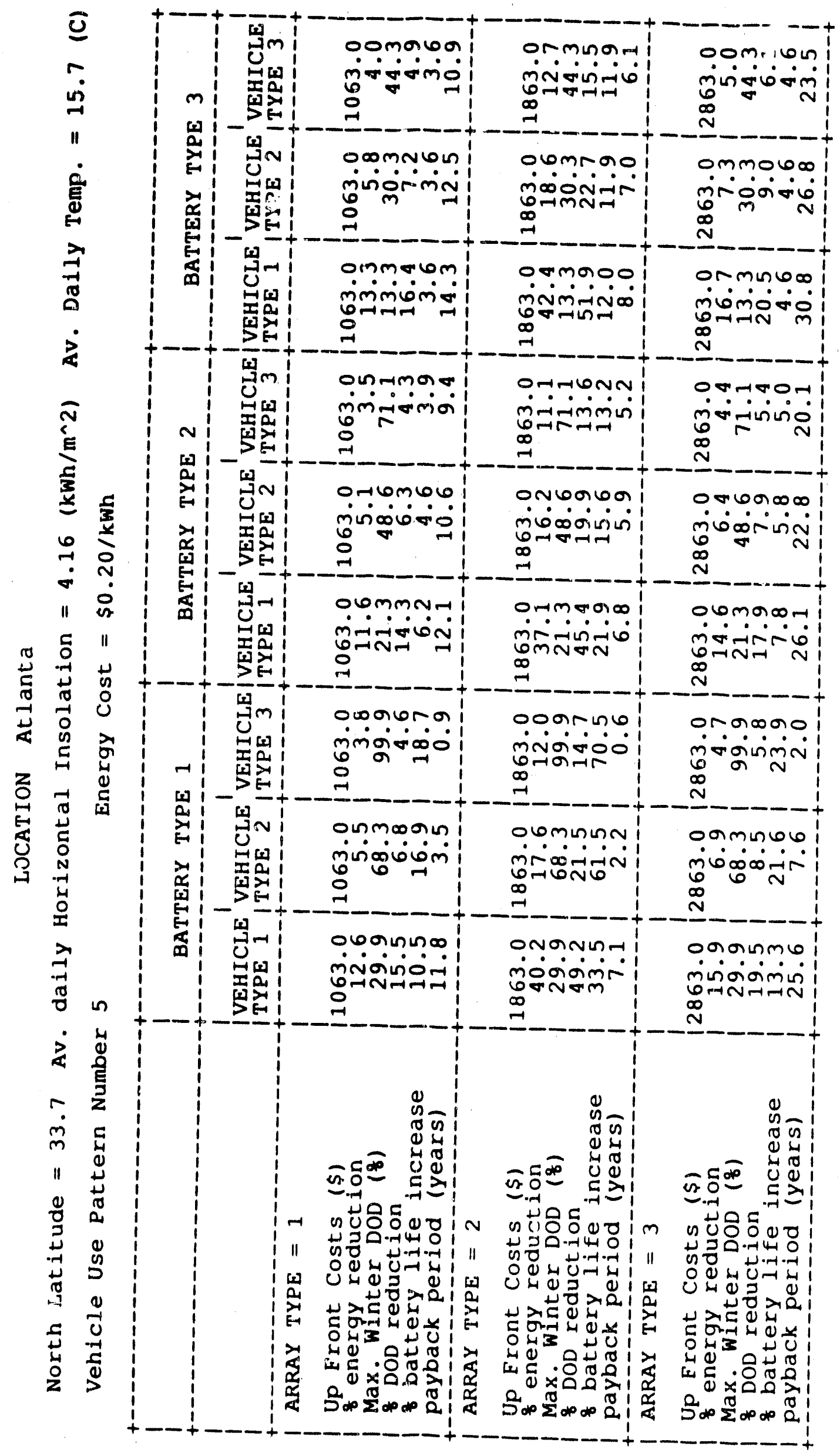




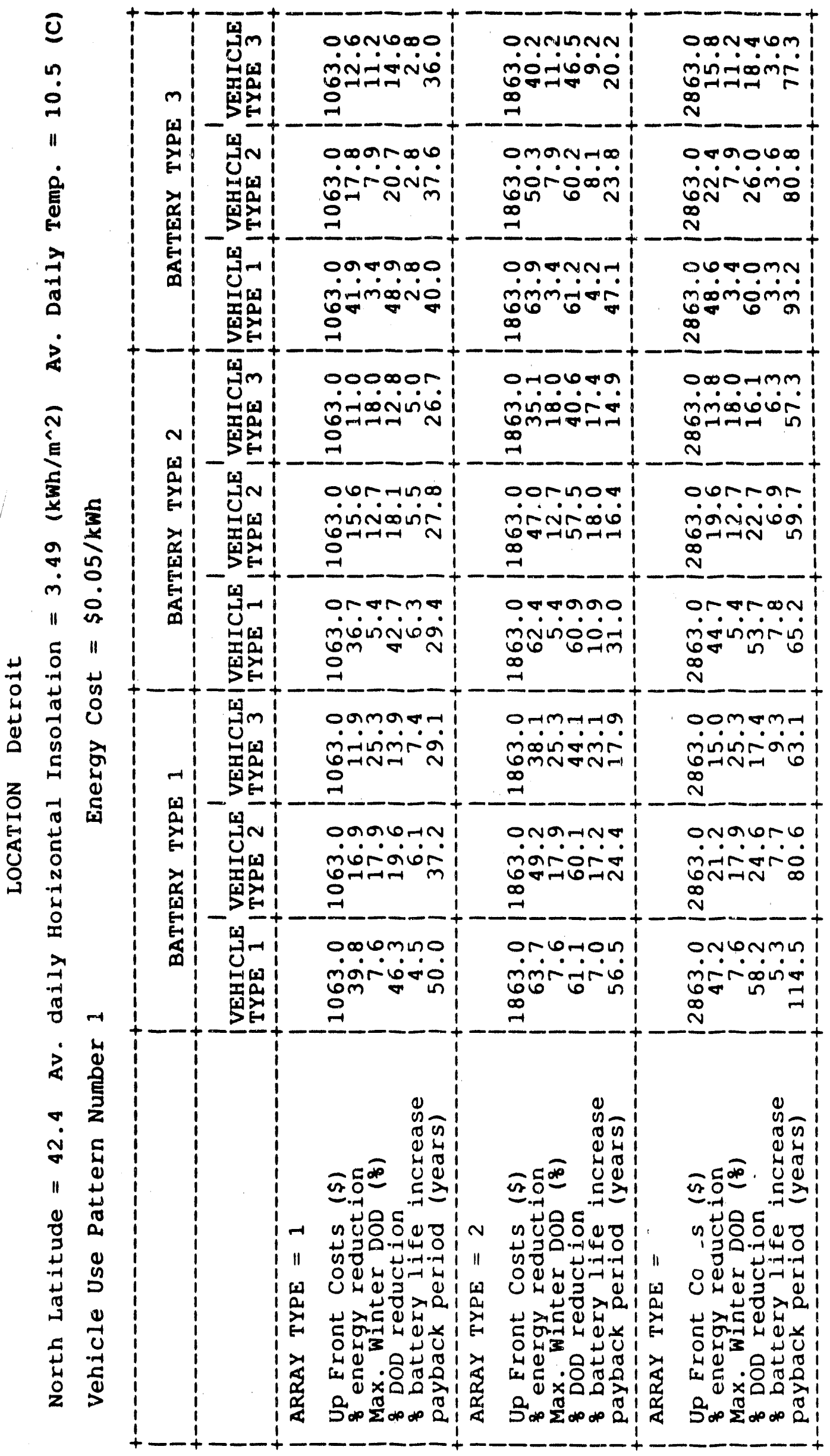




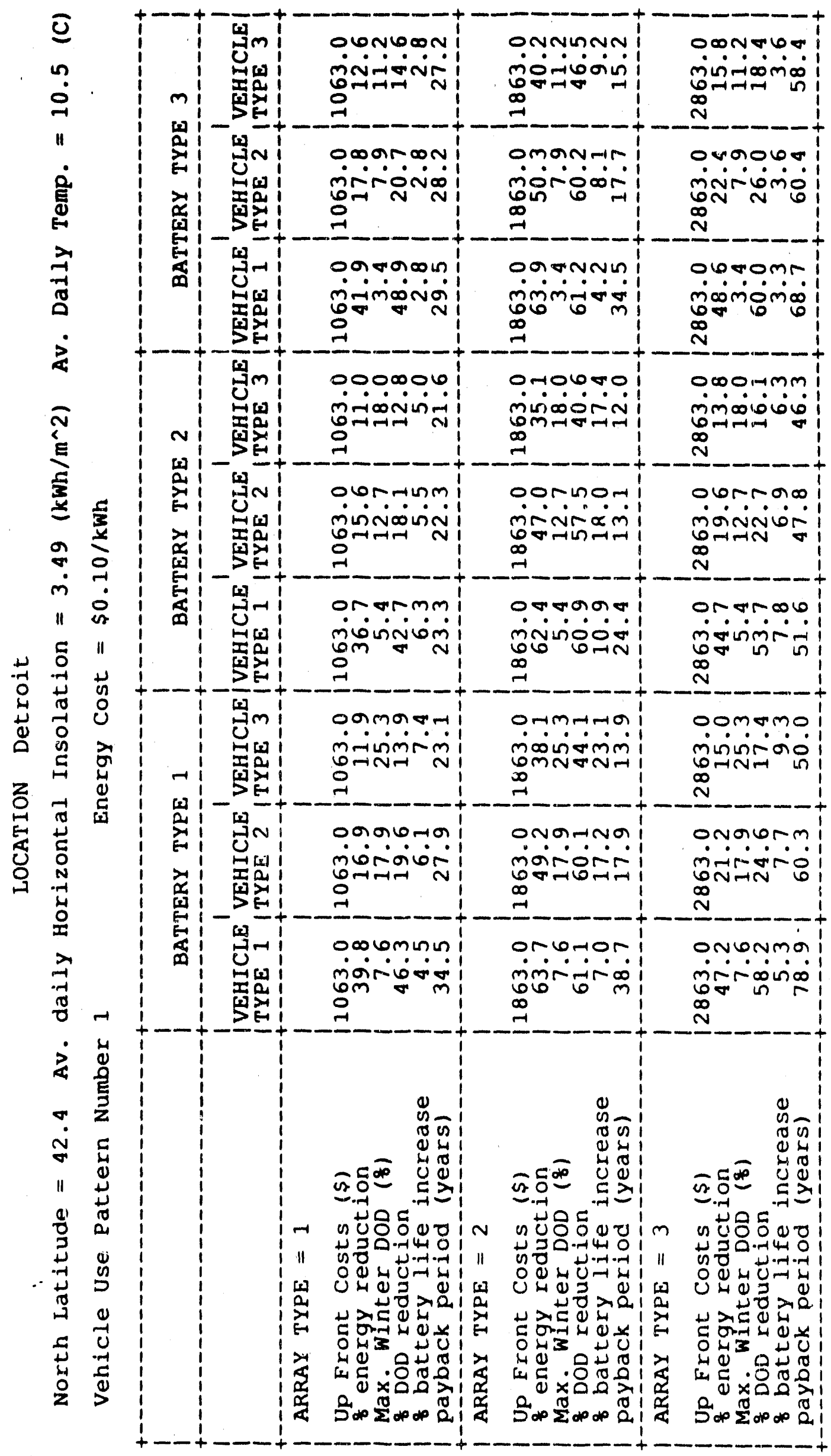




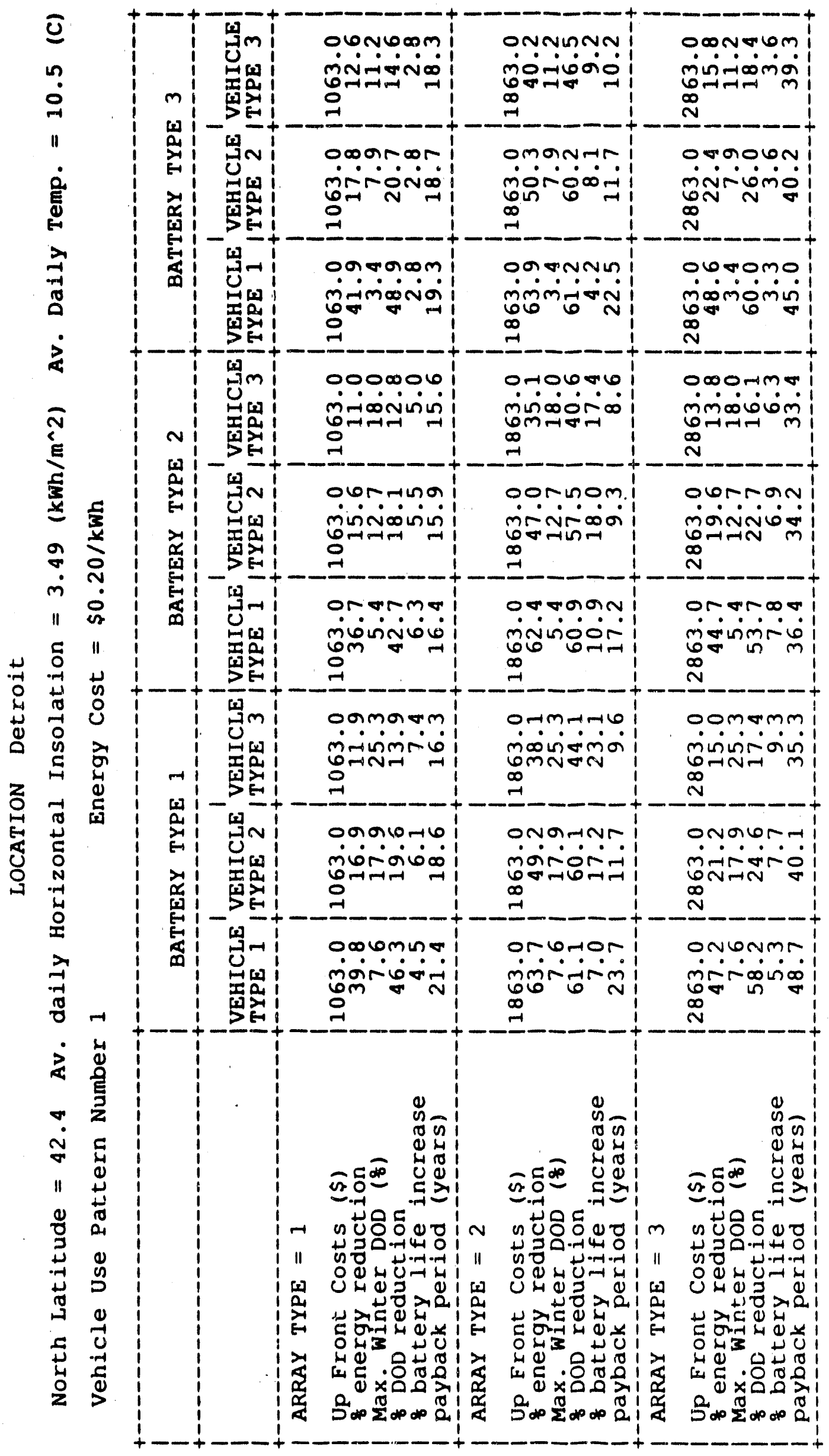




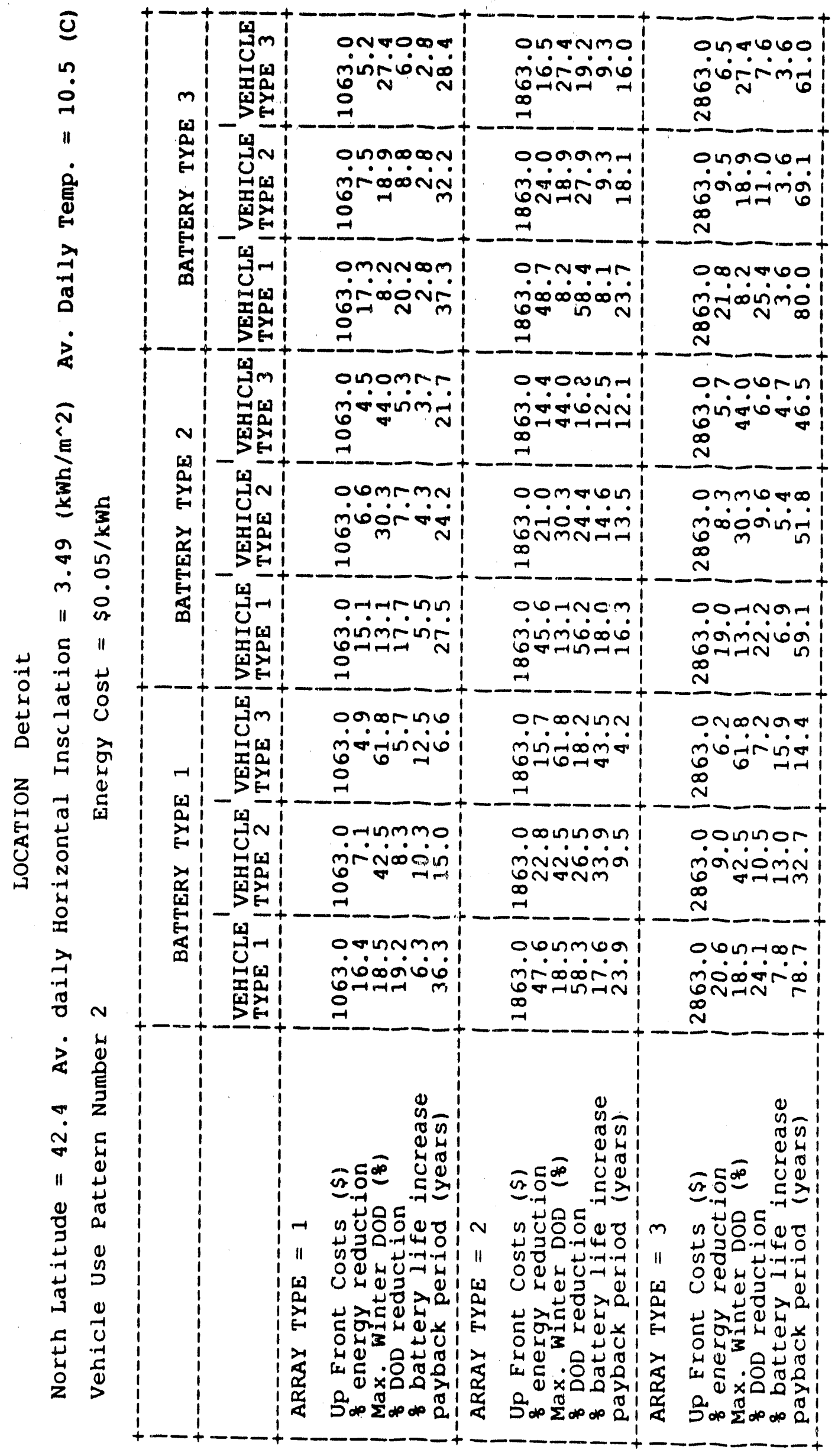




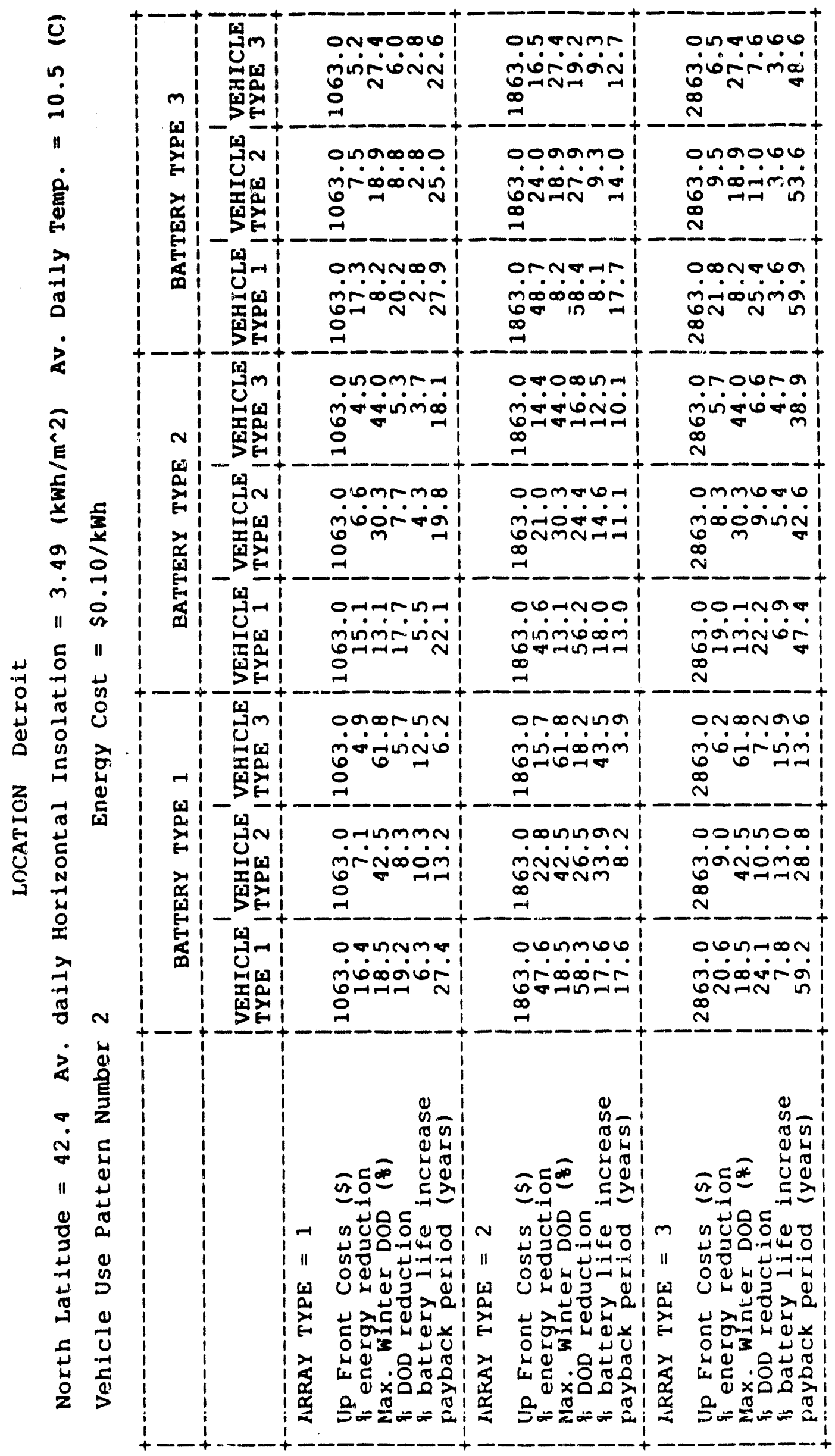




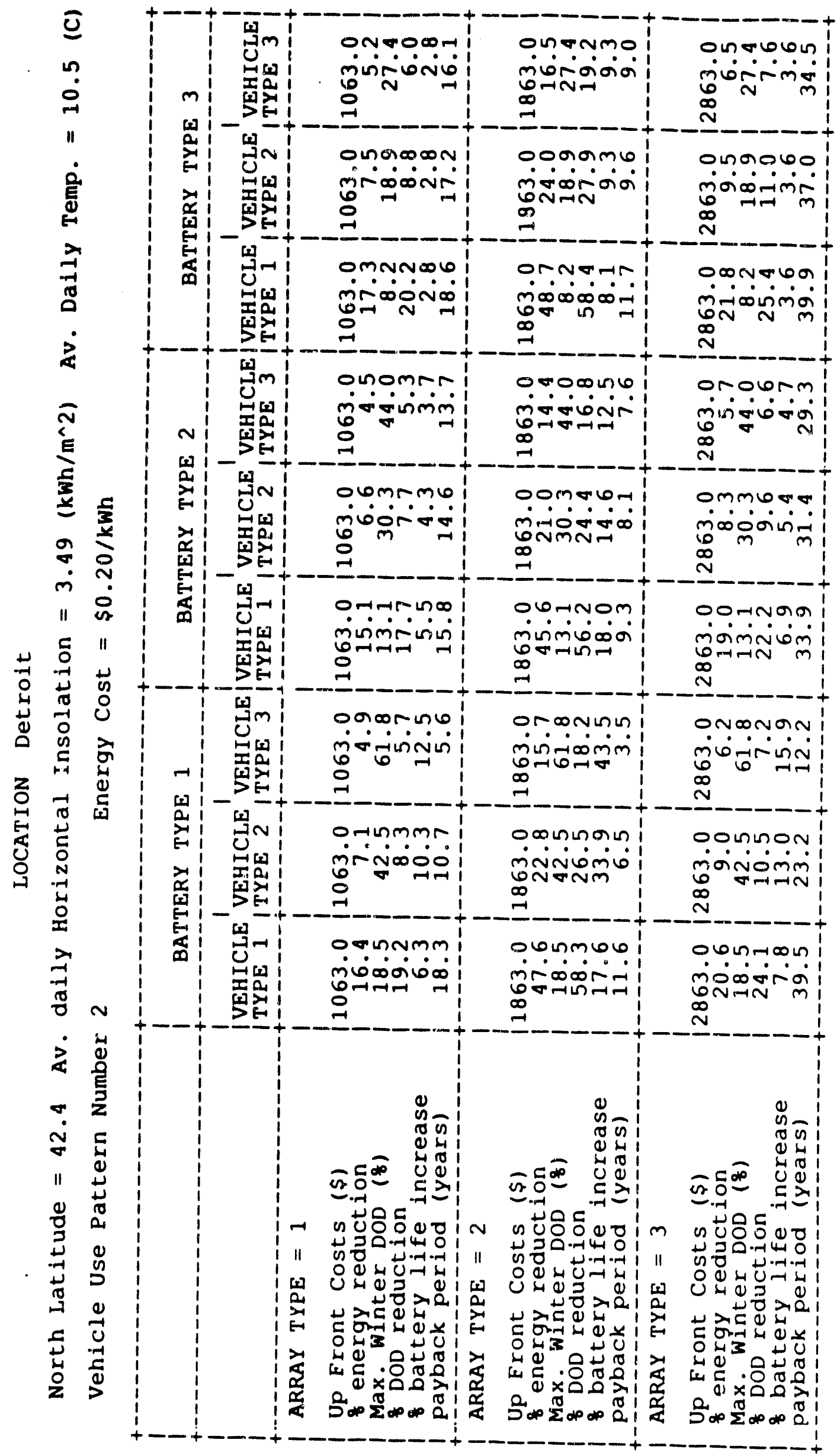

$\underset{1}{\infty}$ 


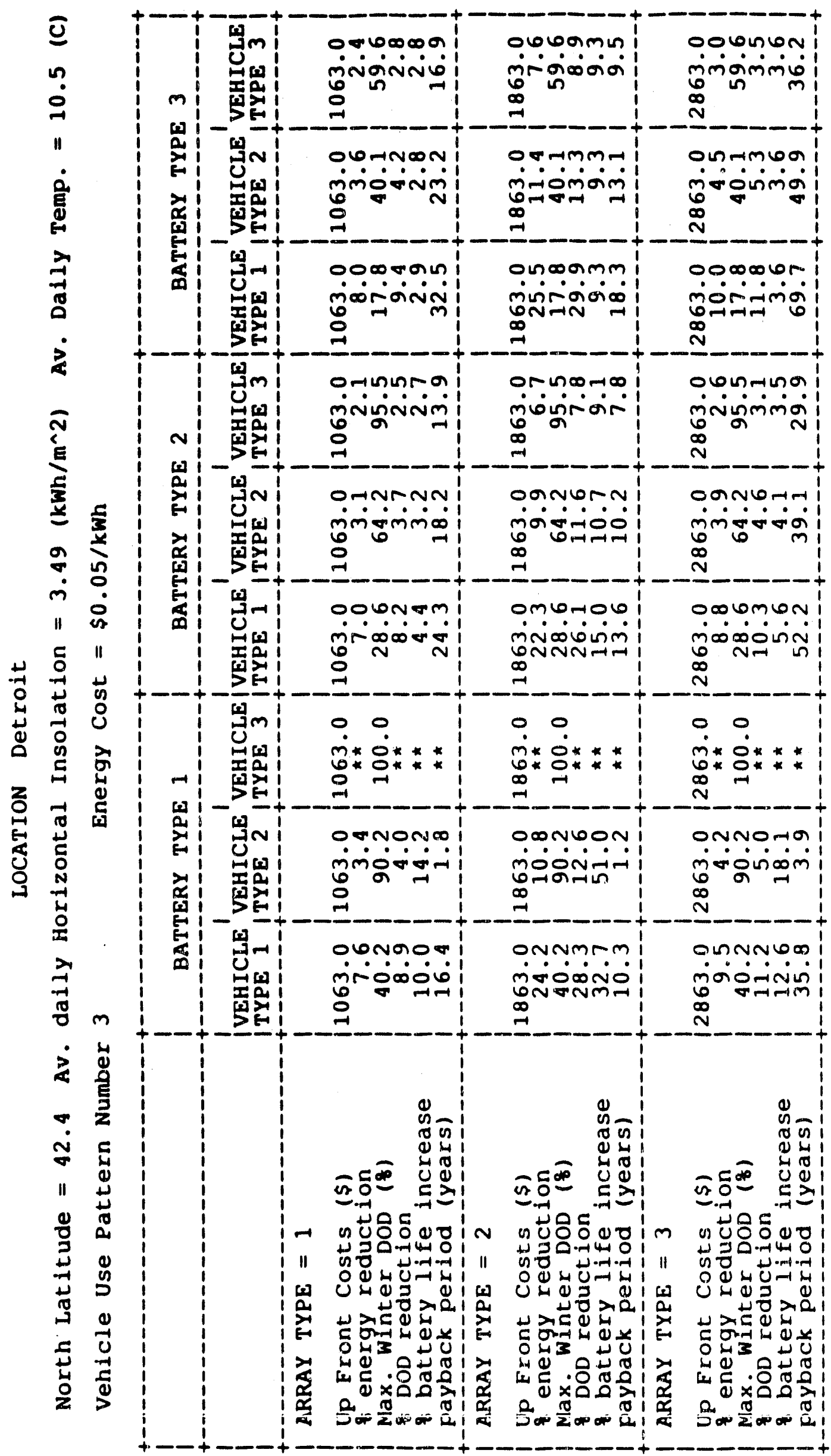




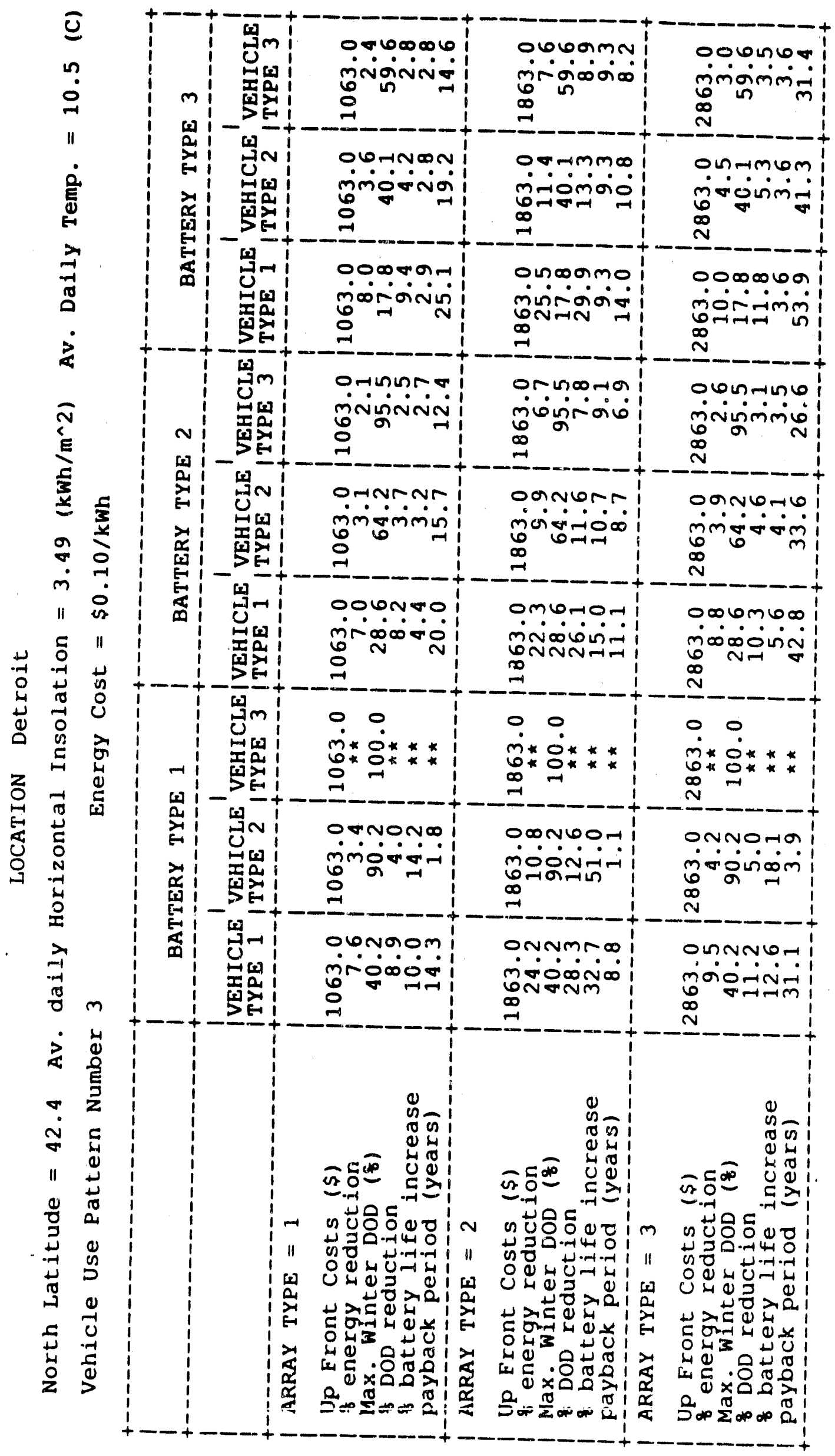




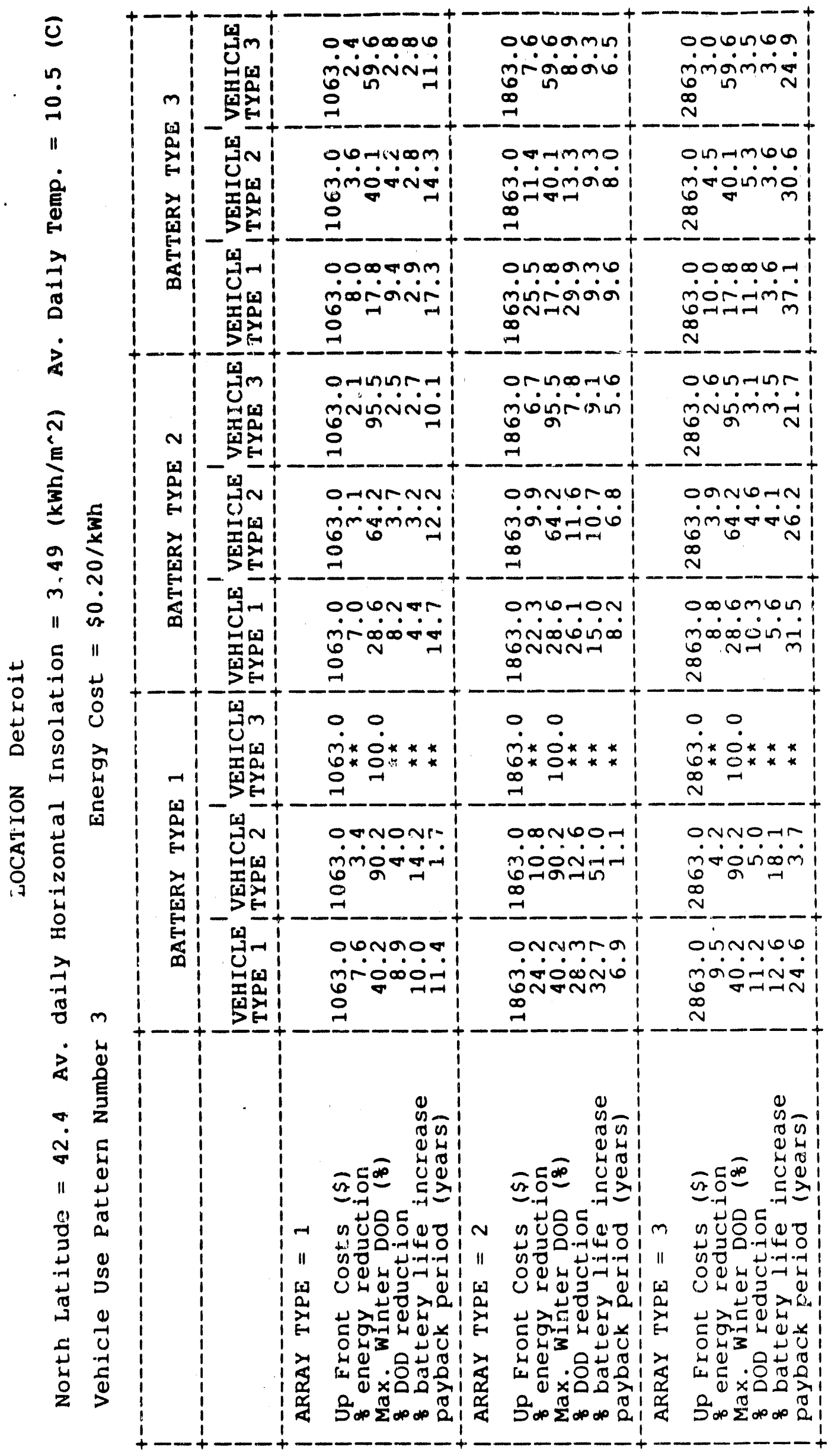




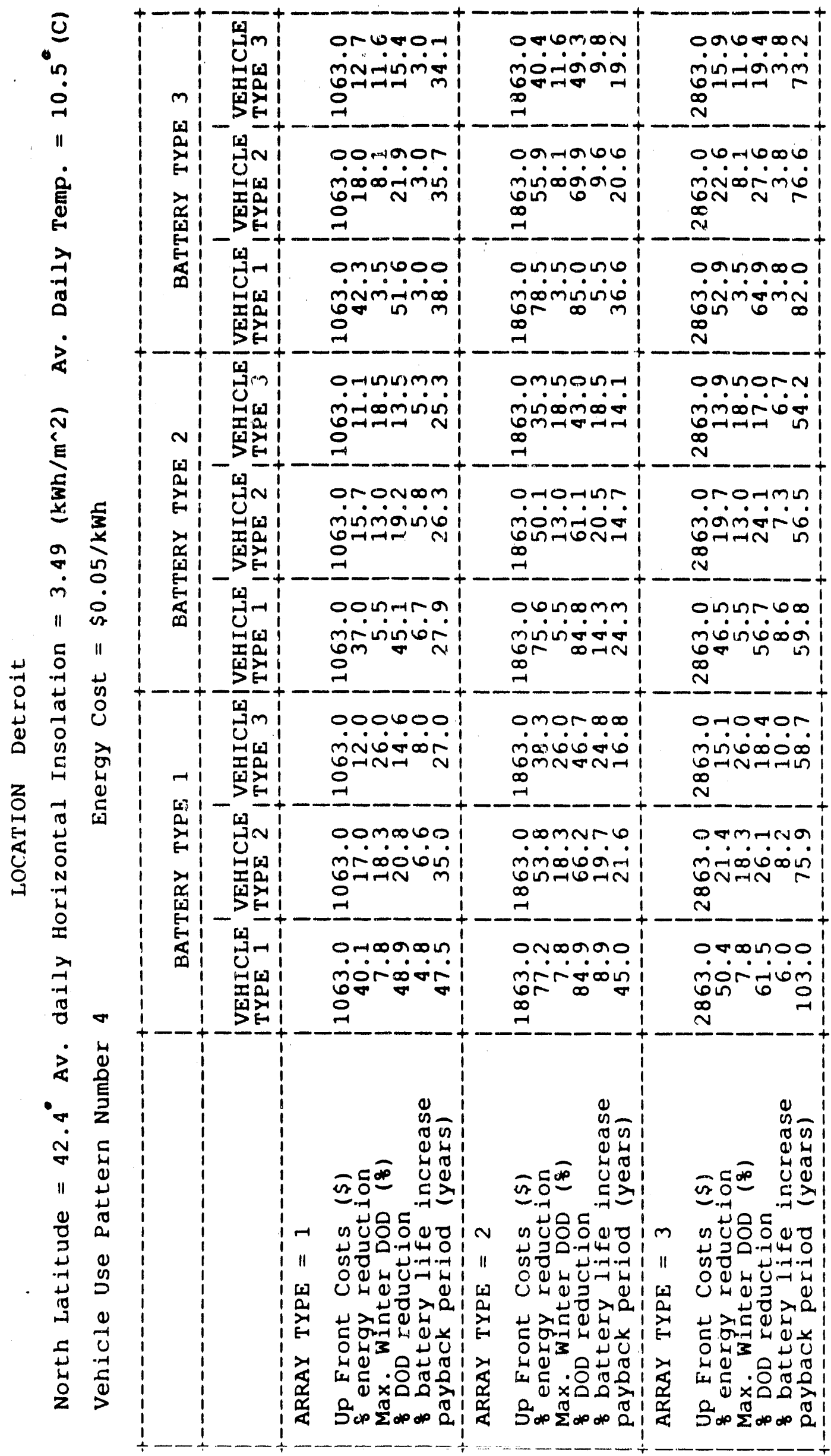




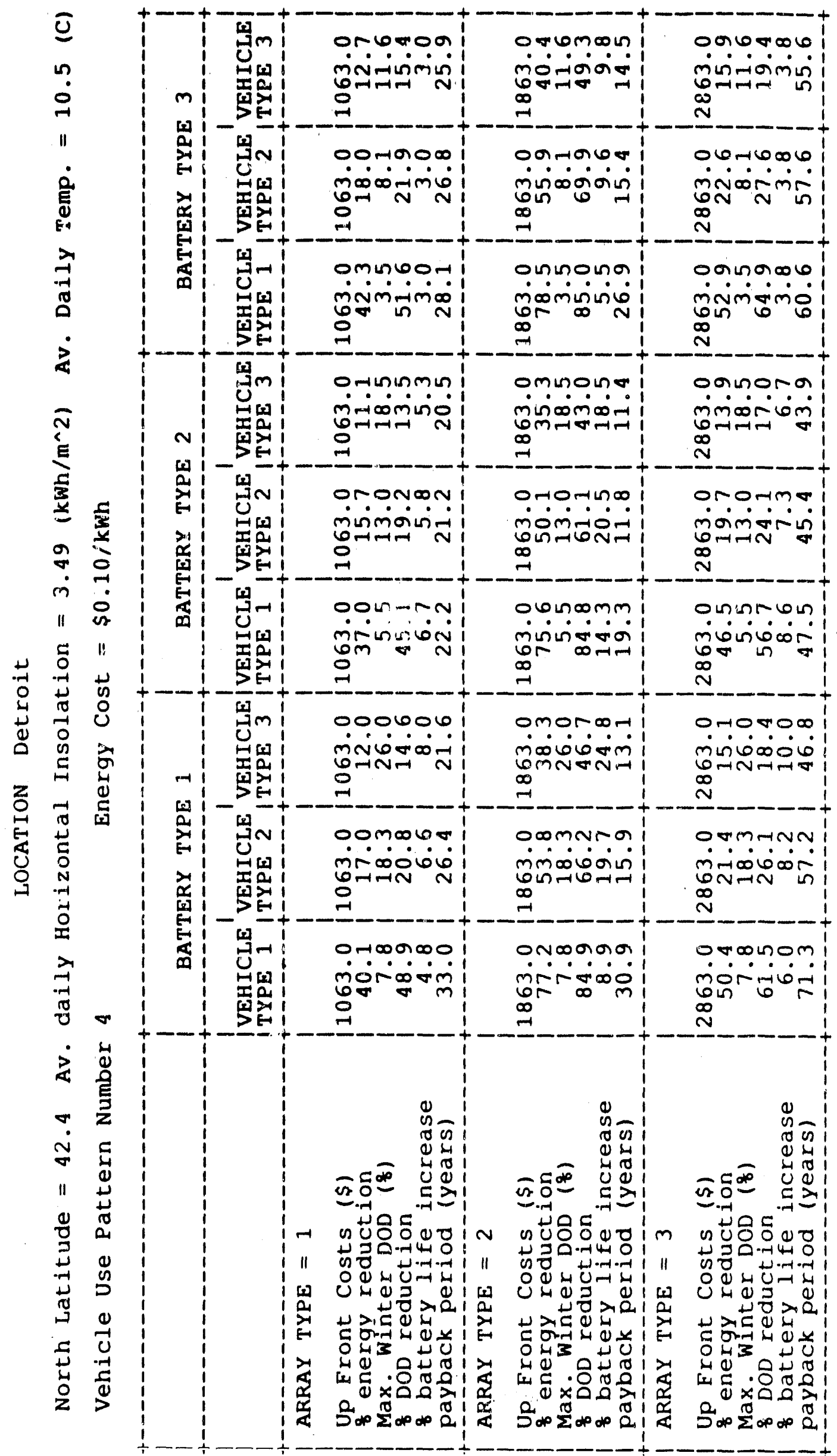

1
5
0
1
1 


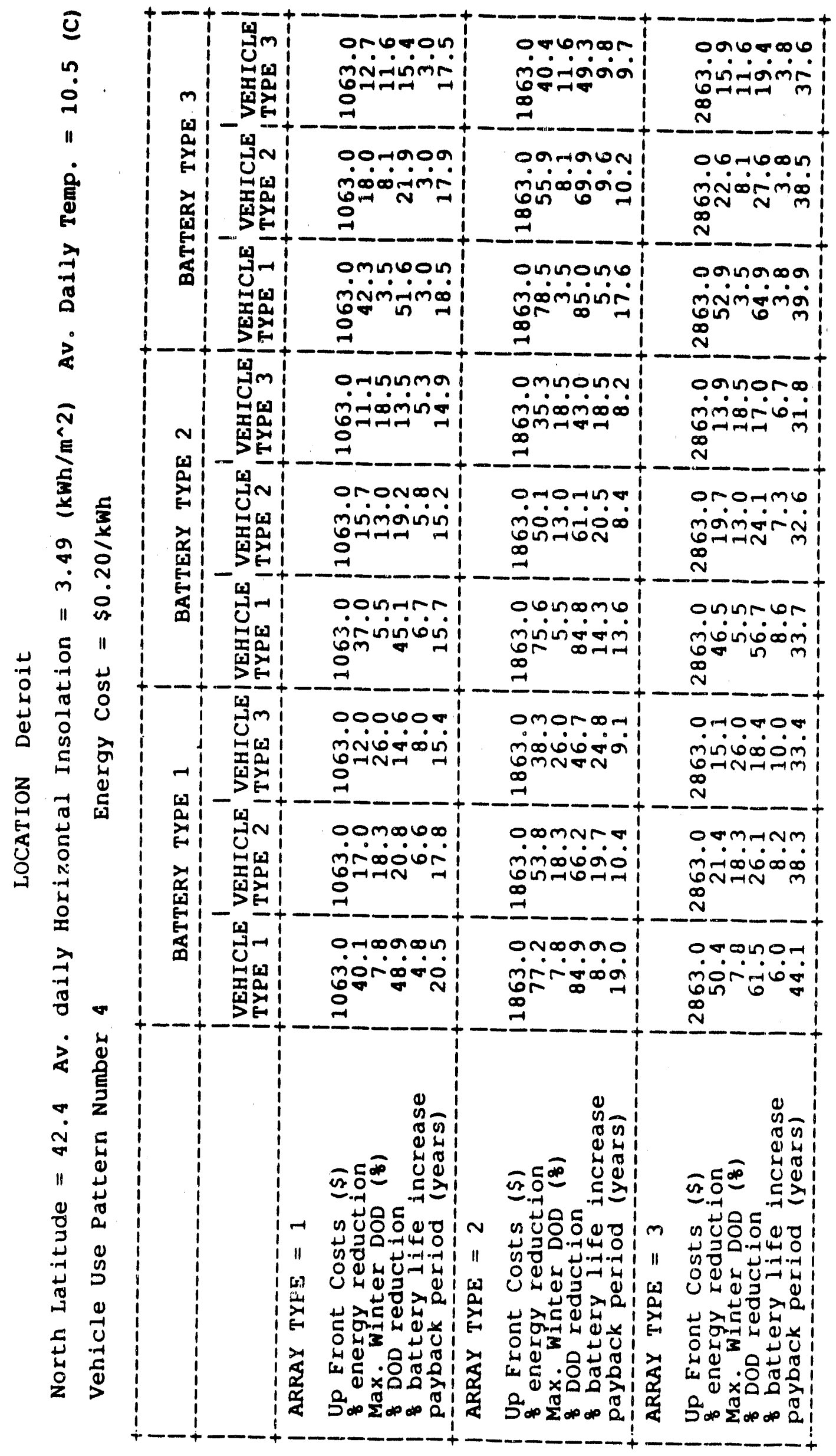




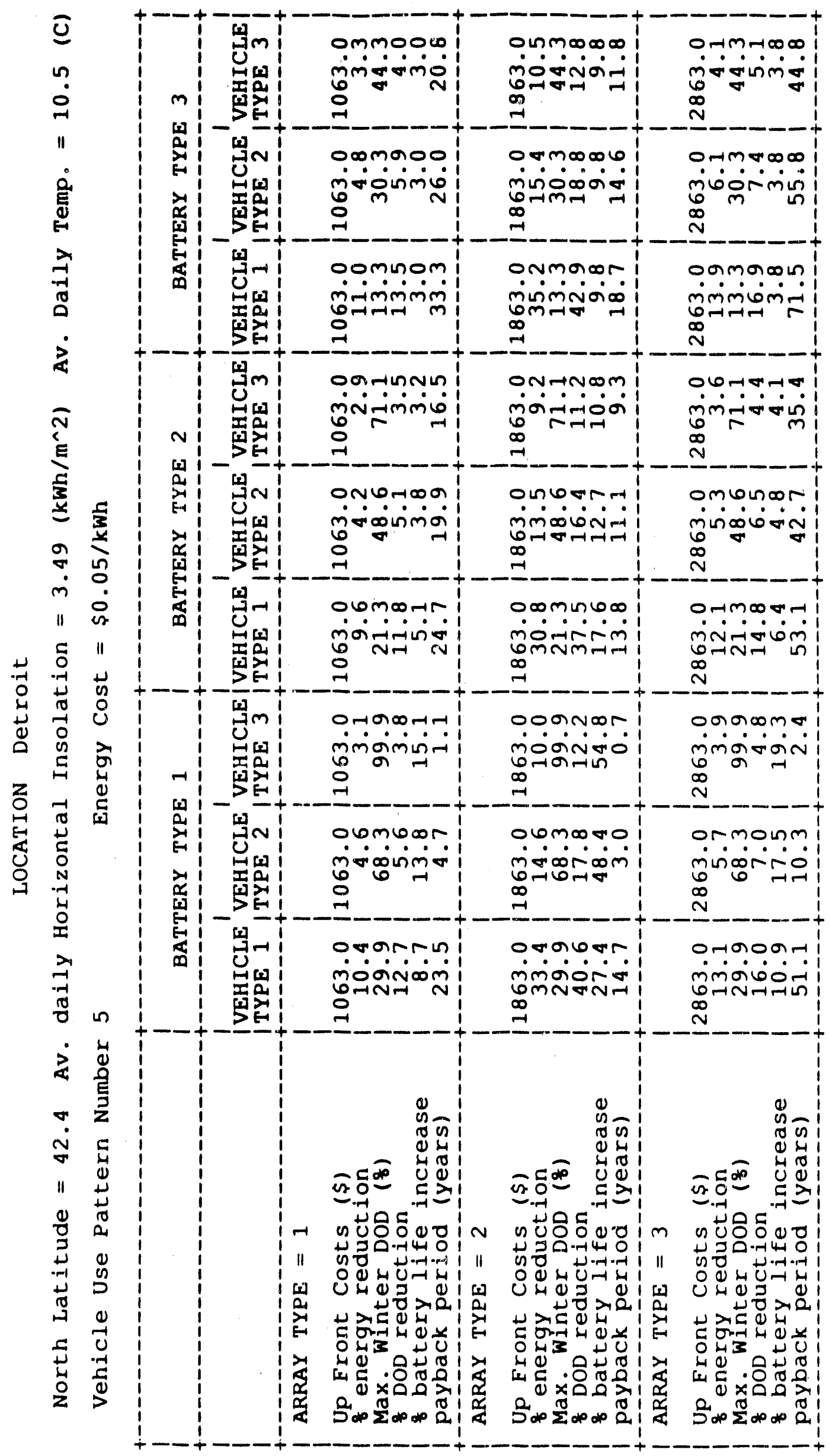




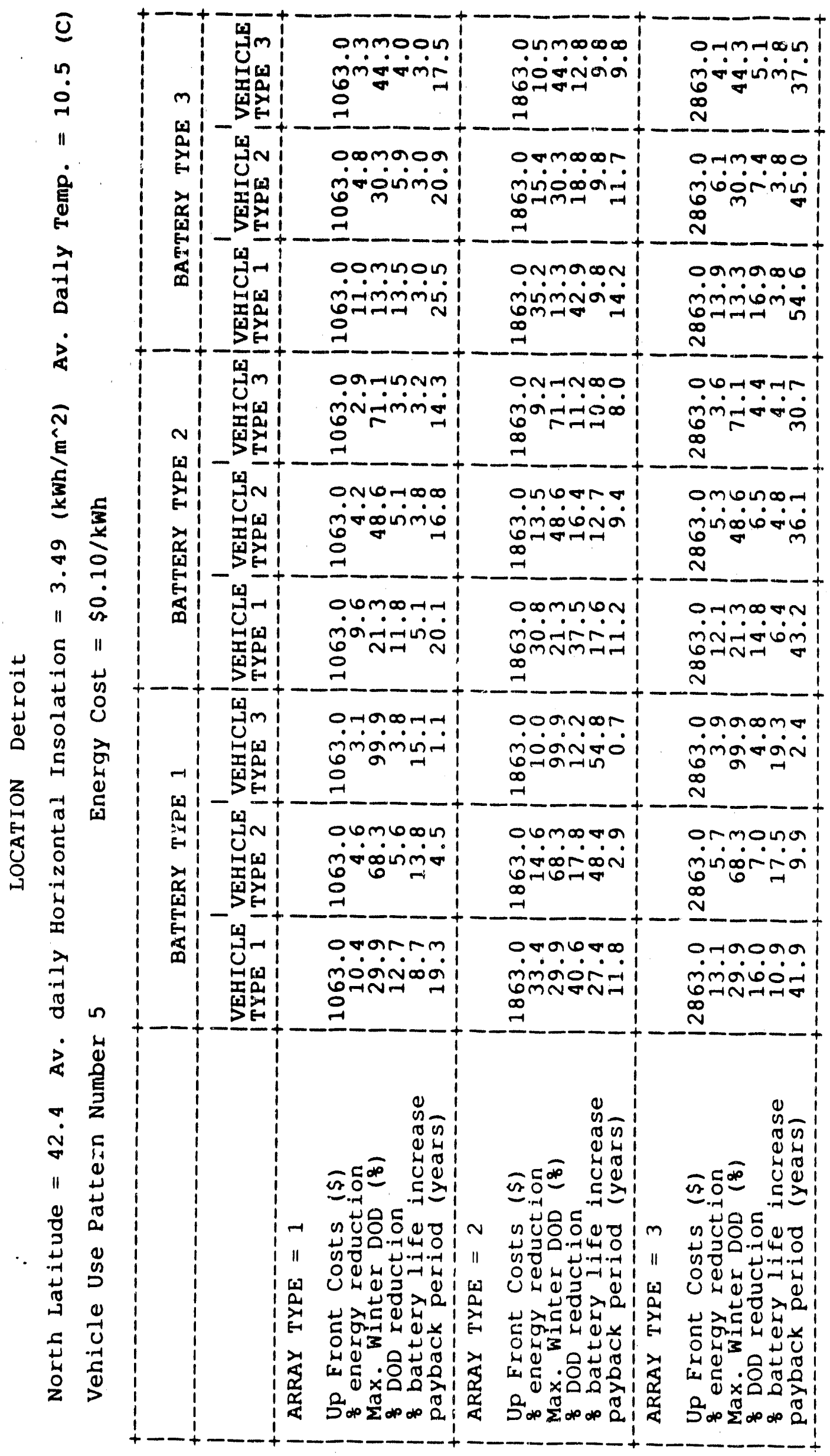




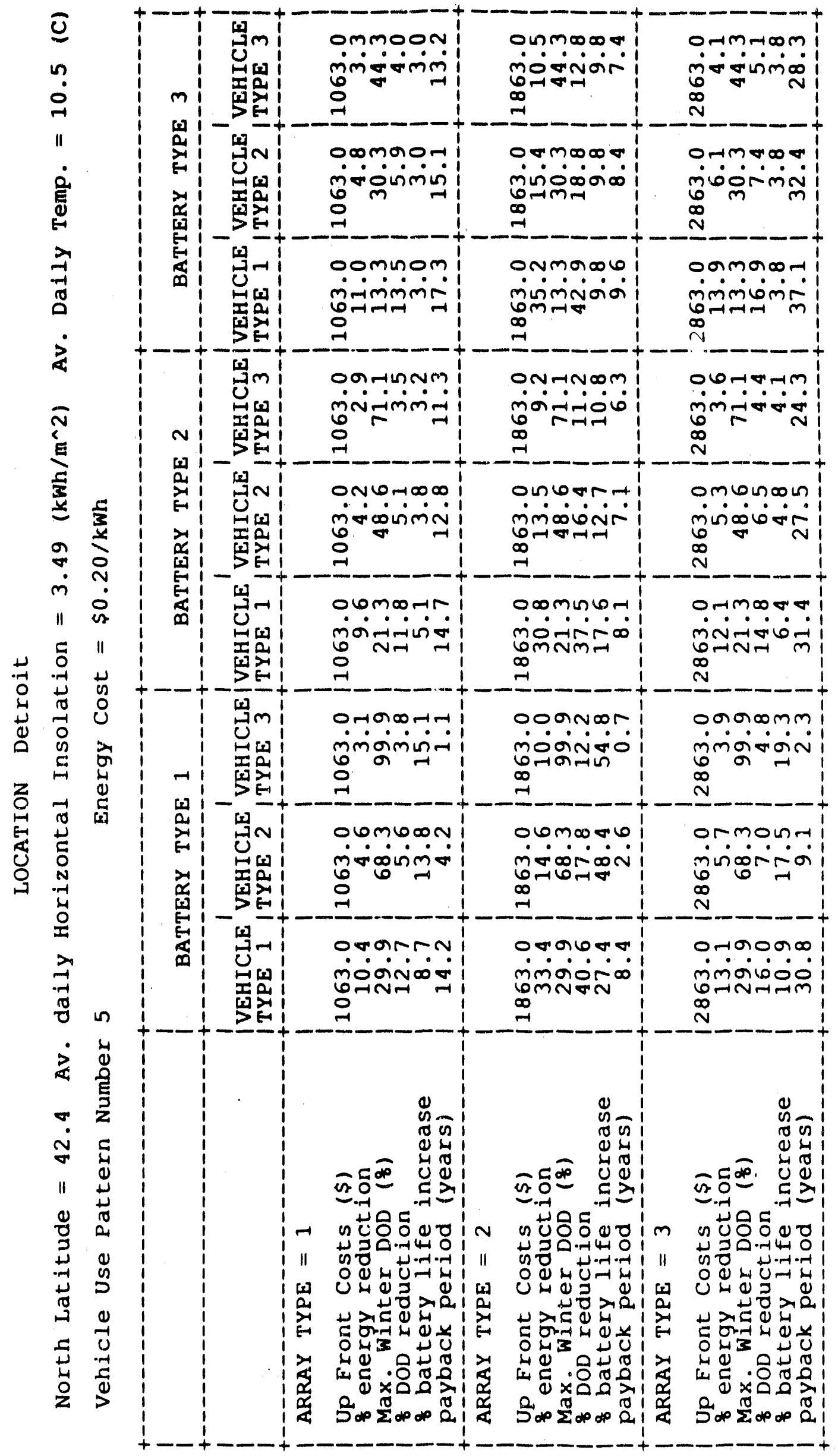




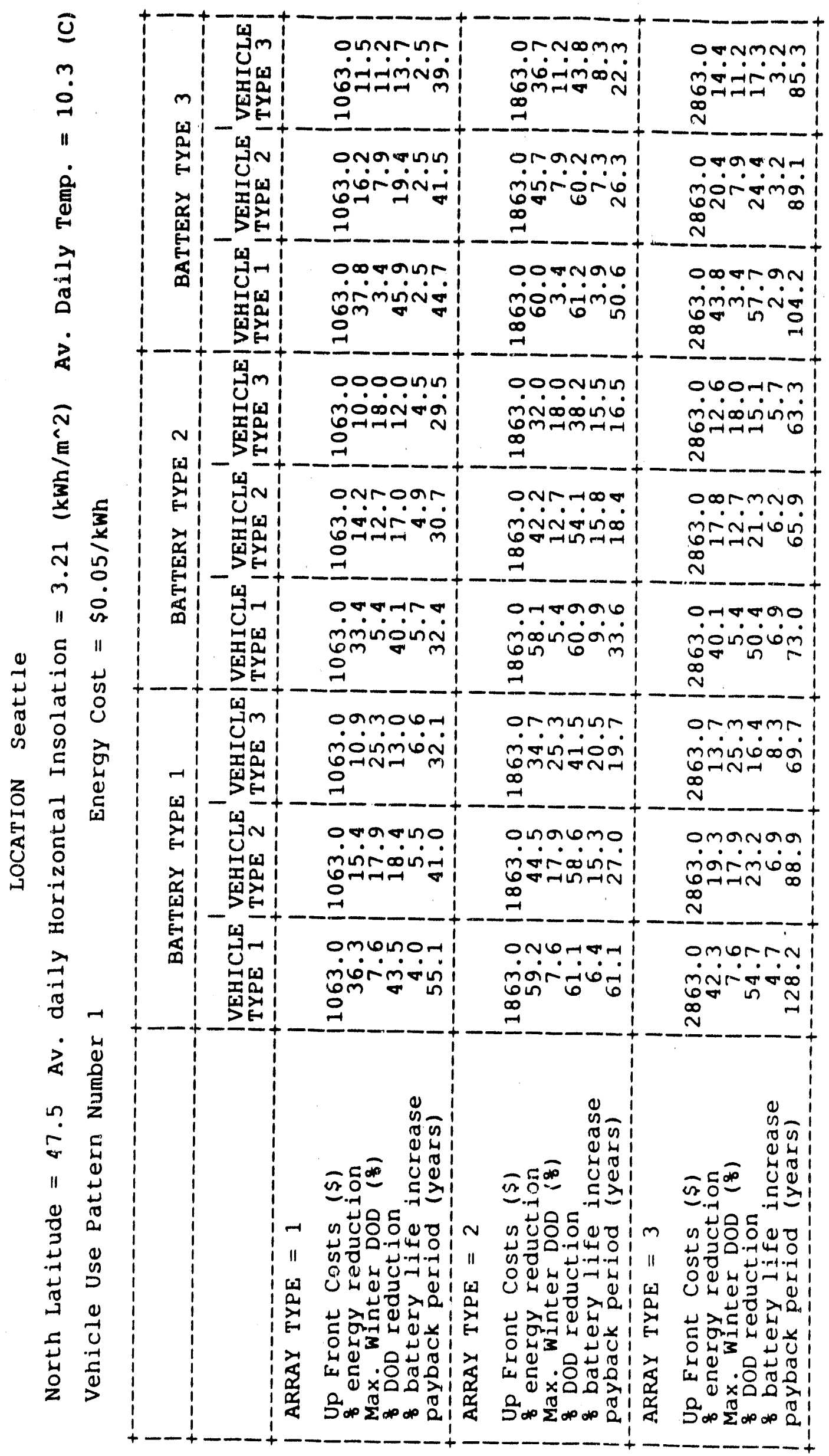




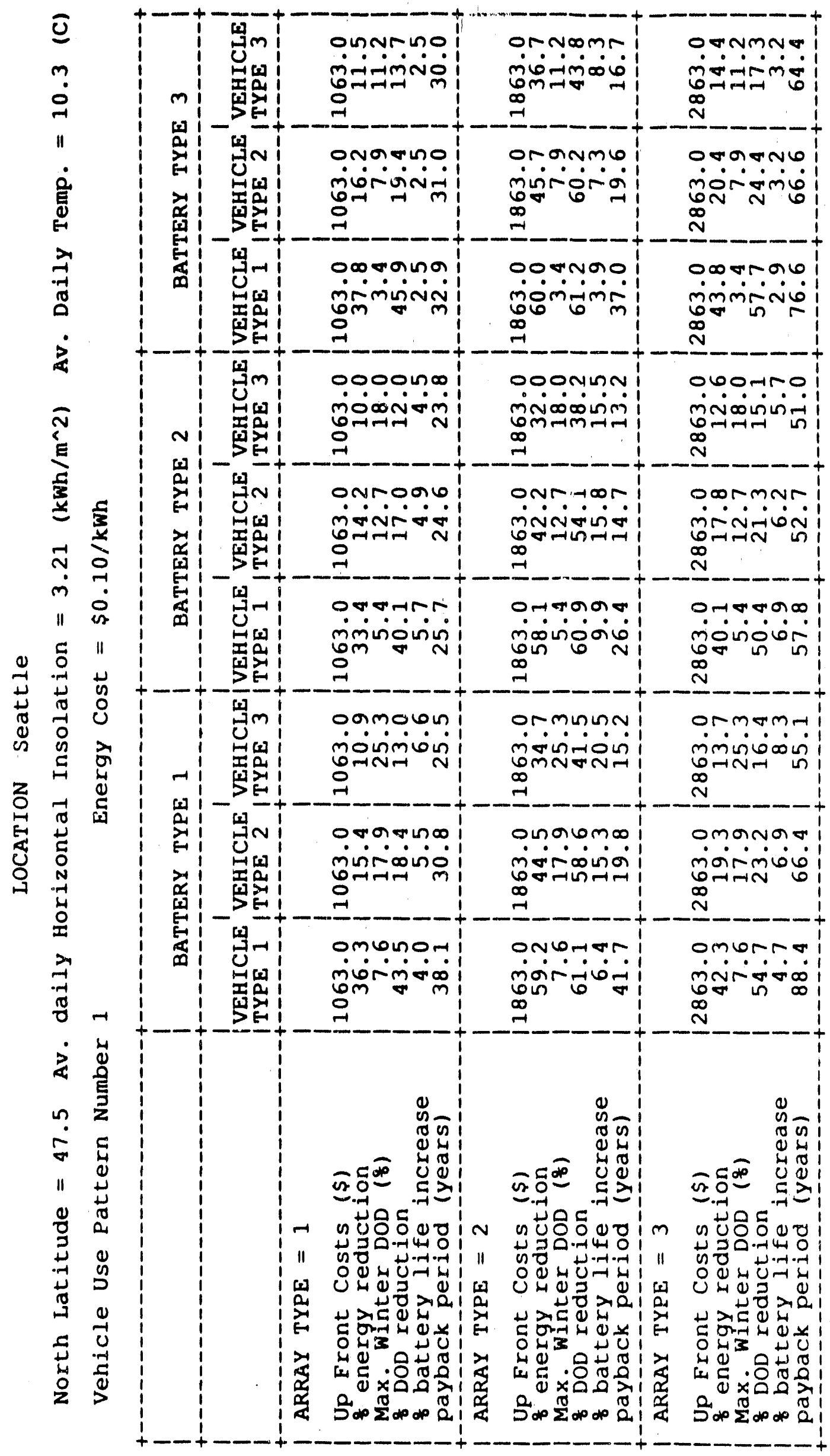




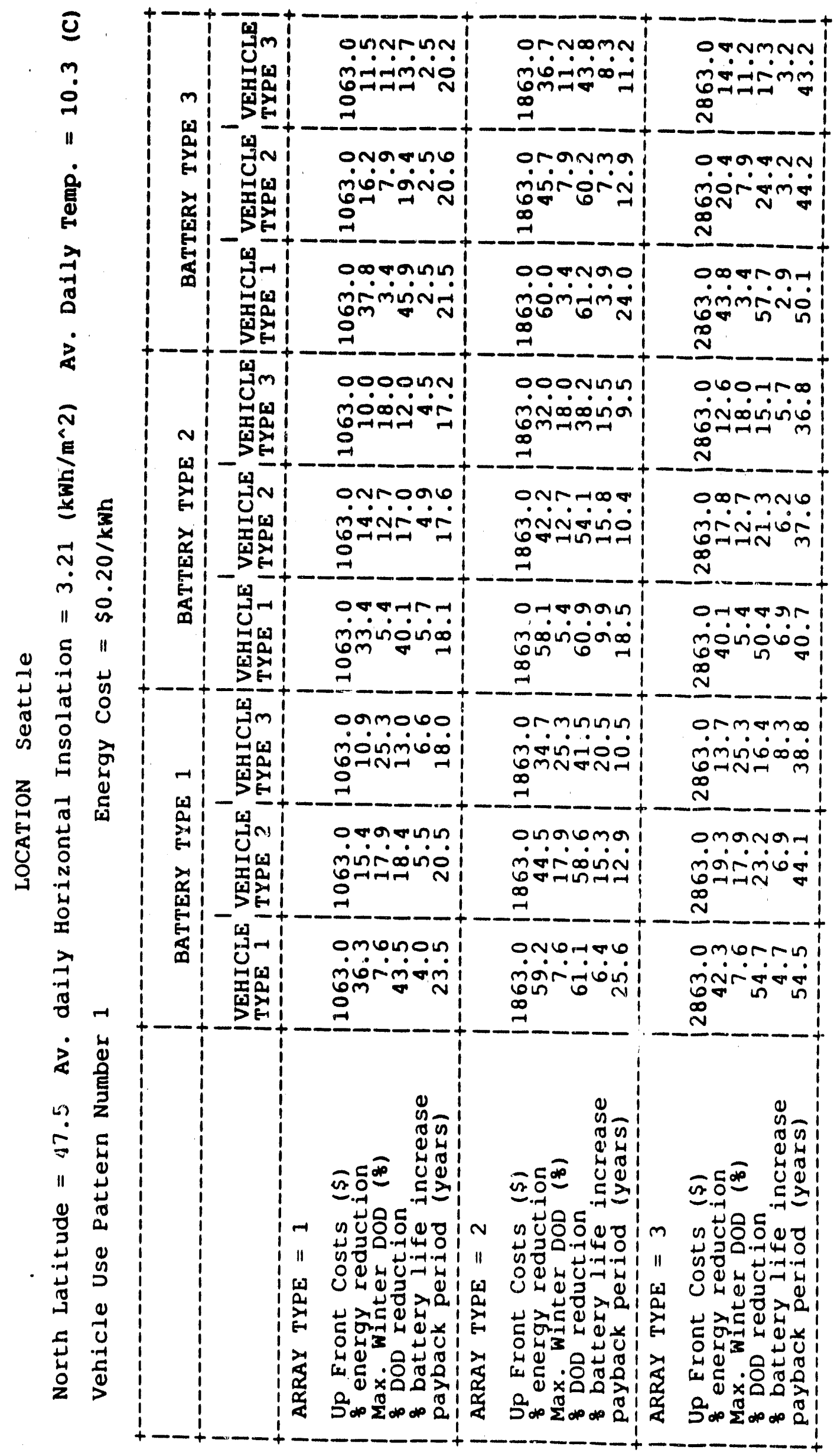




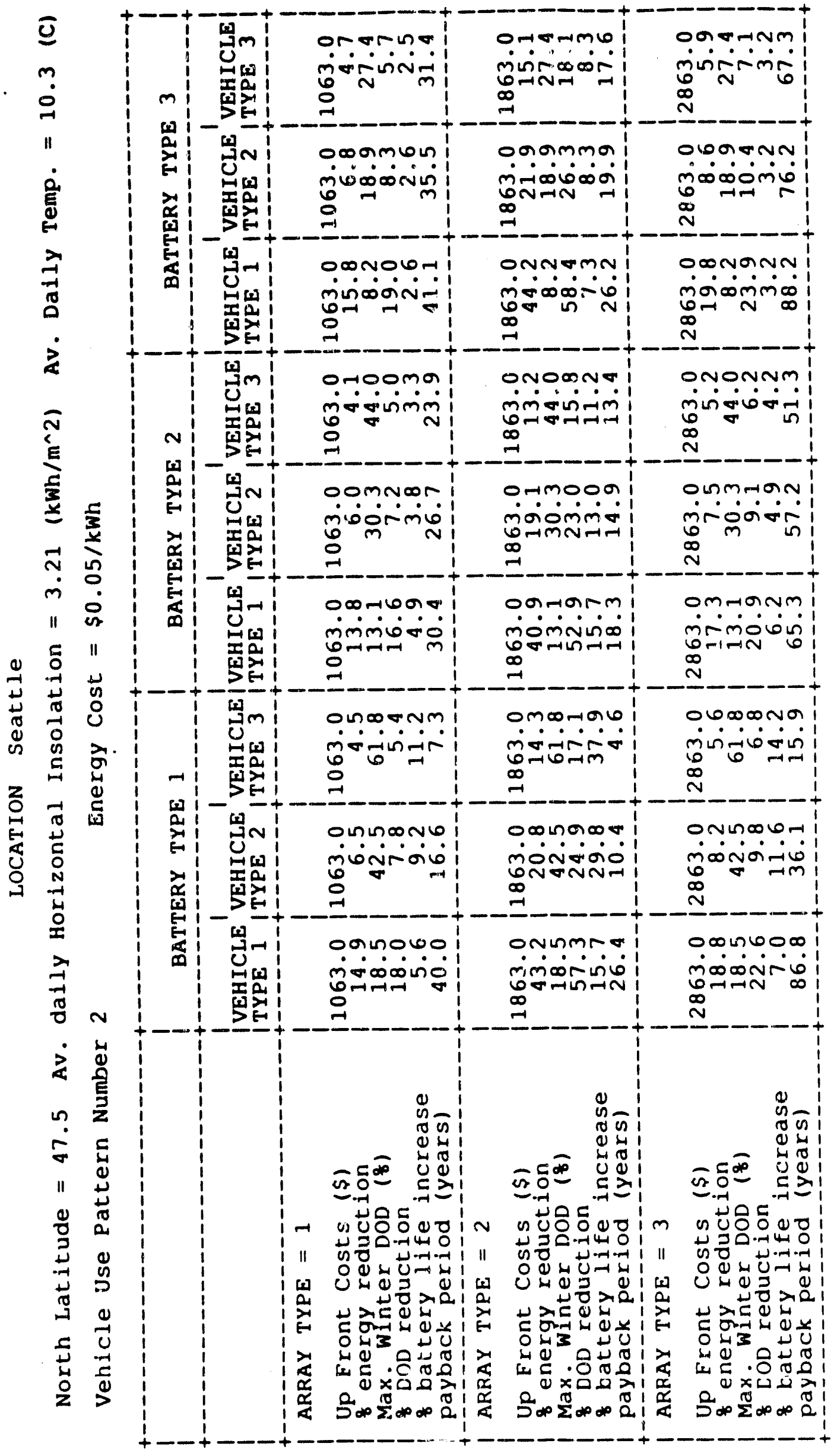




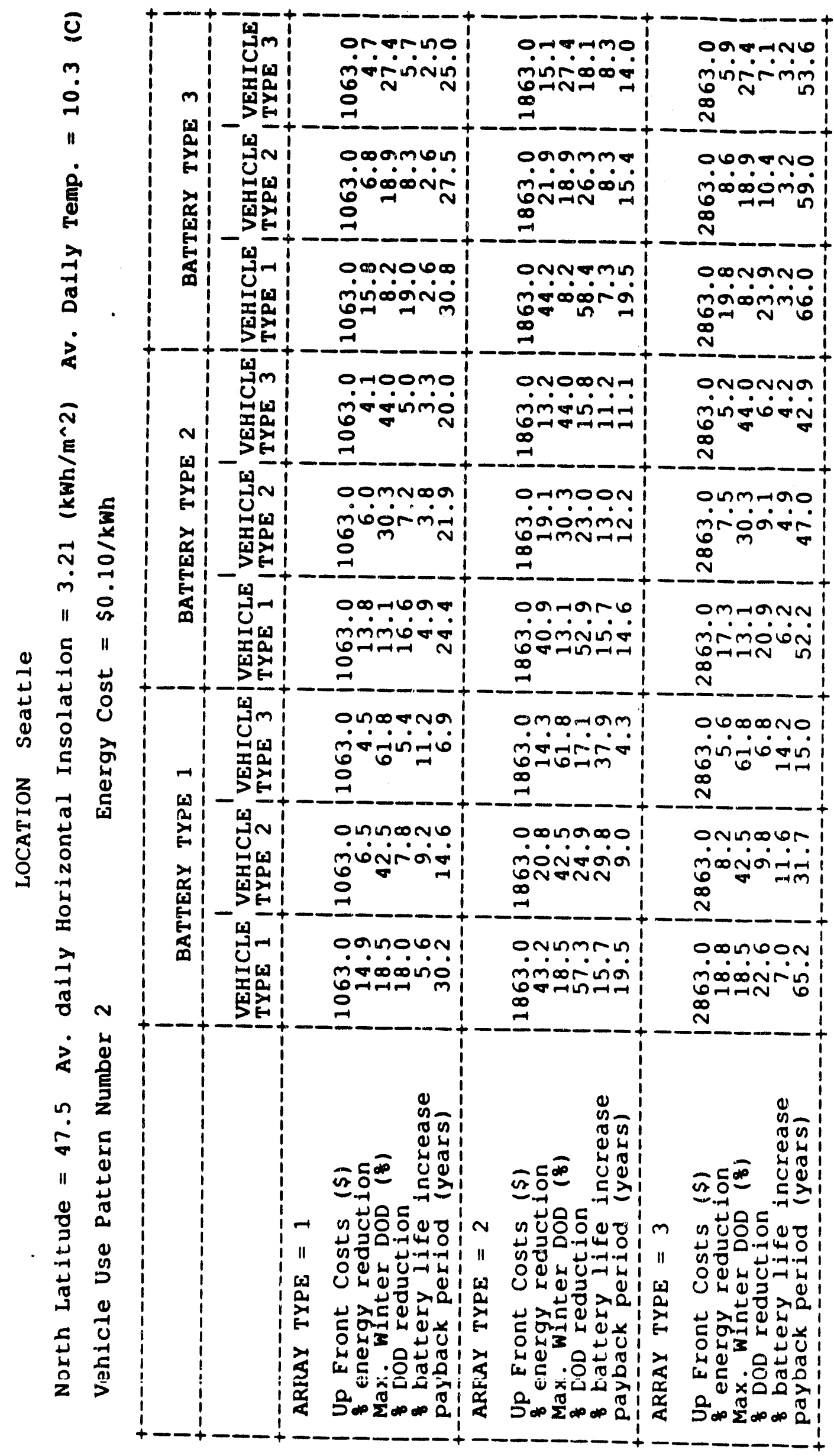




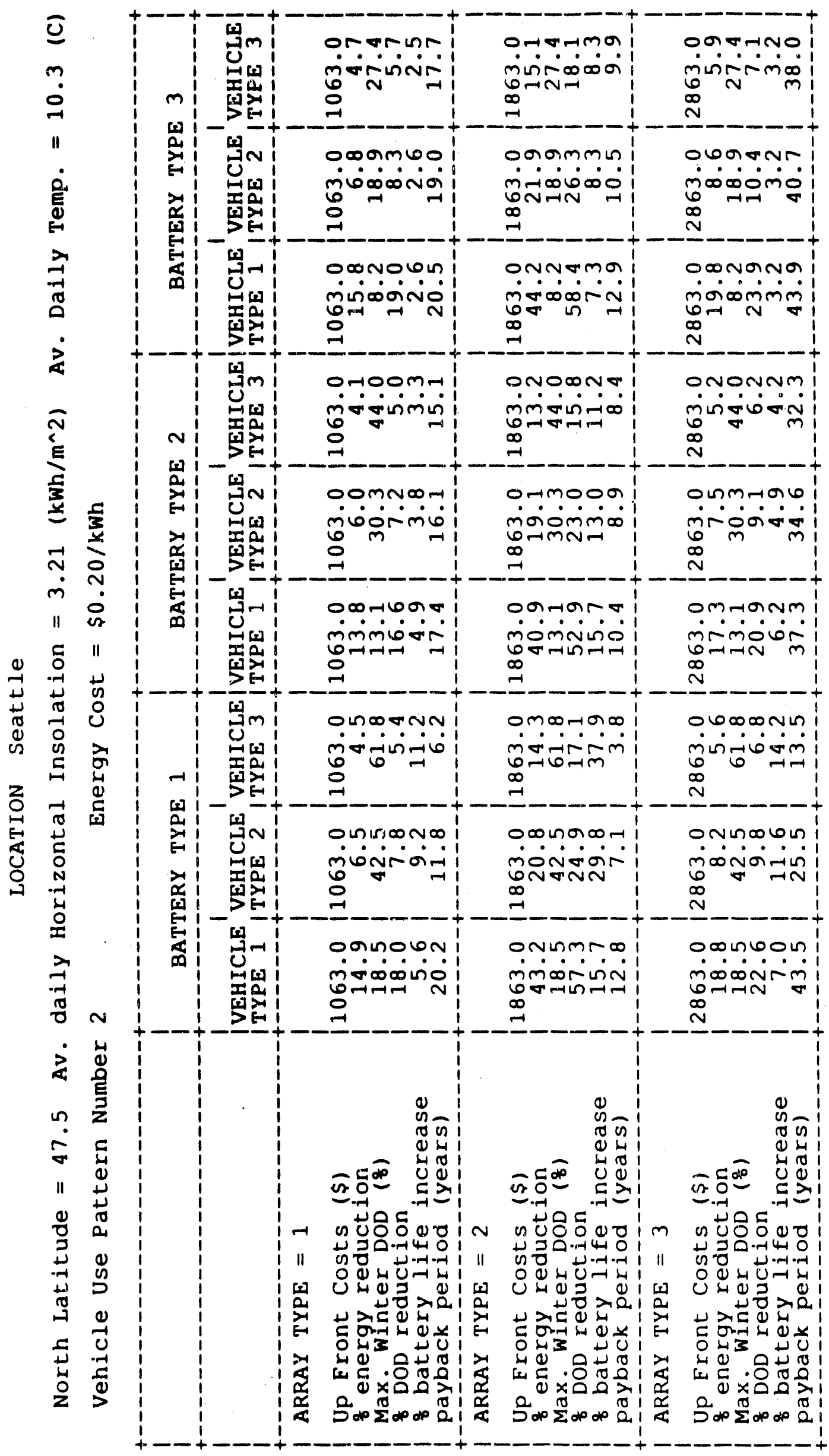

$\frac{1}{9}$ 


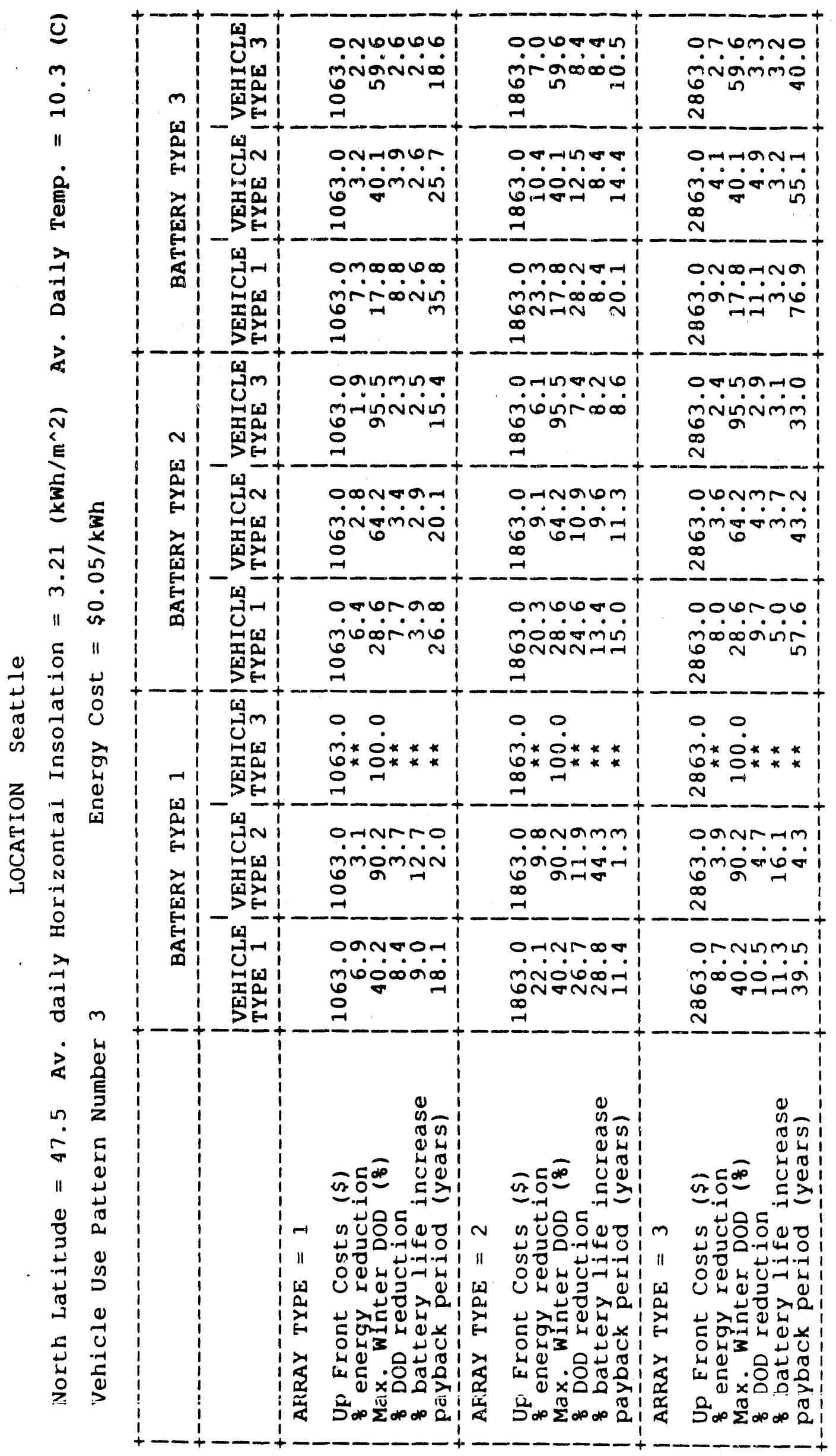




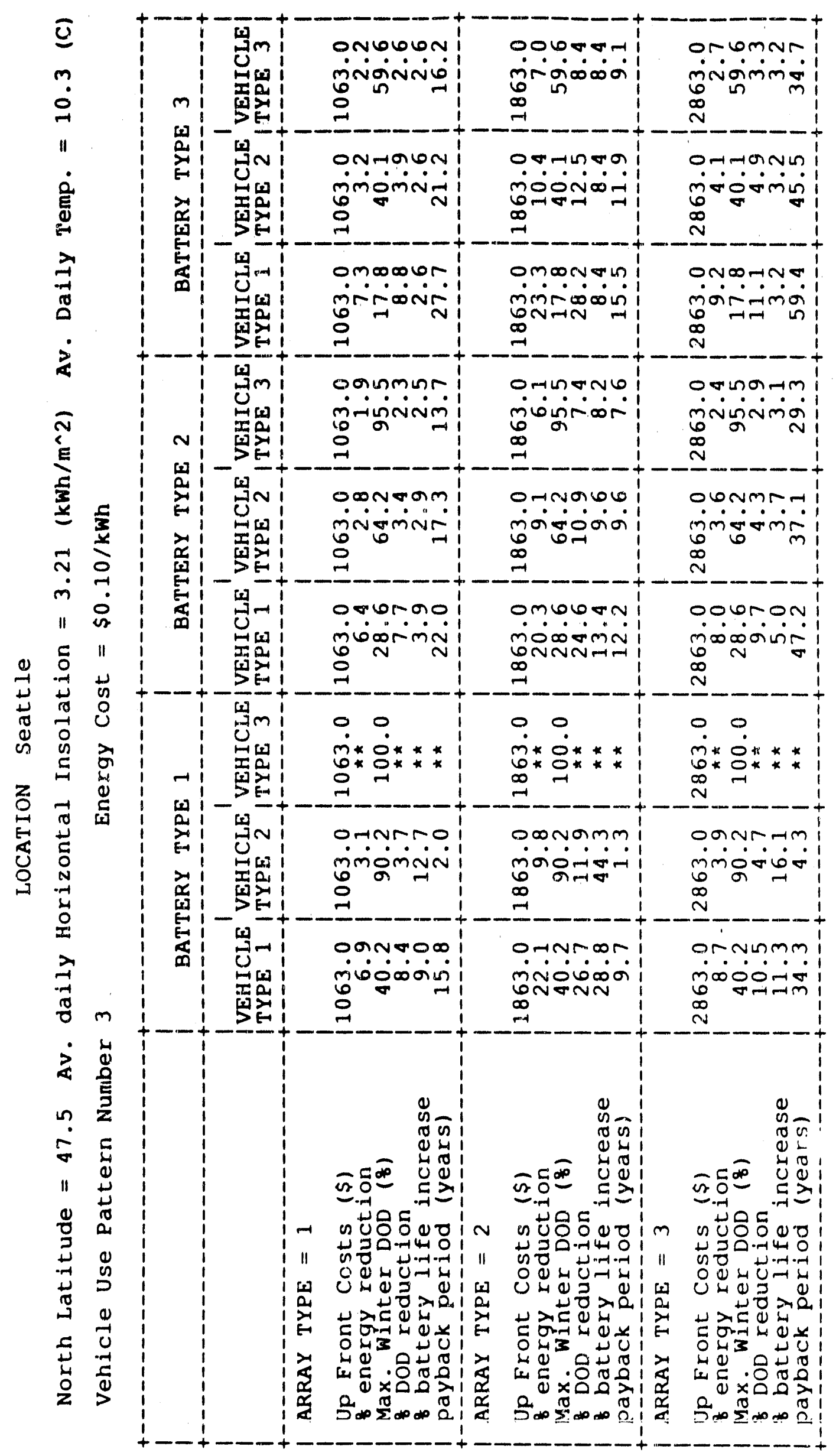




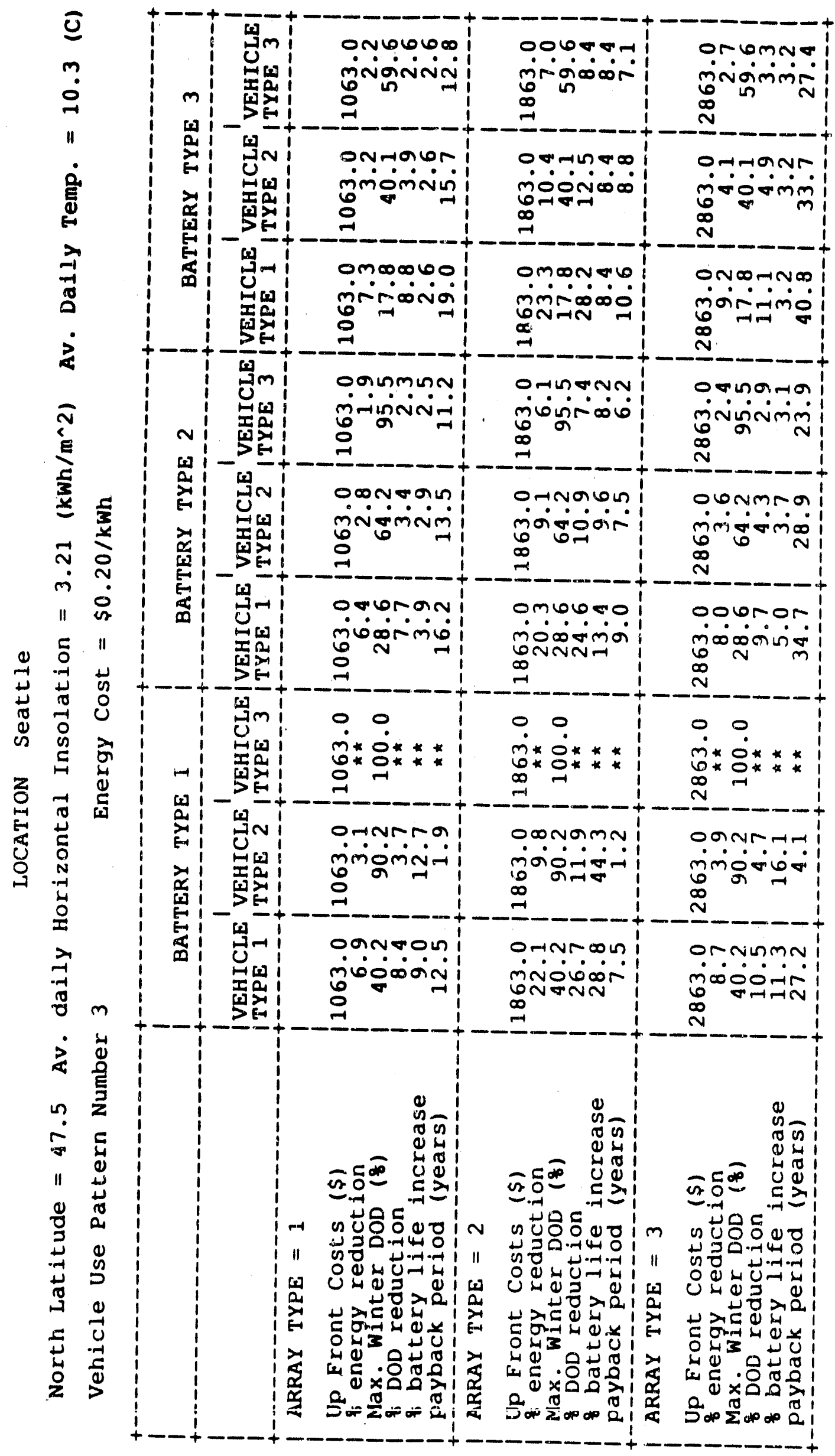




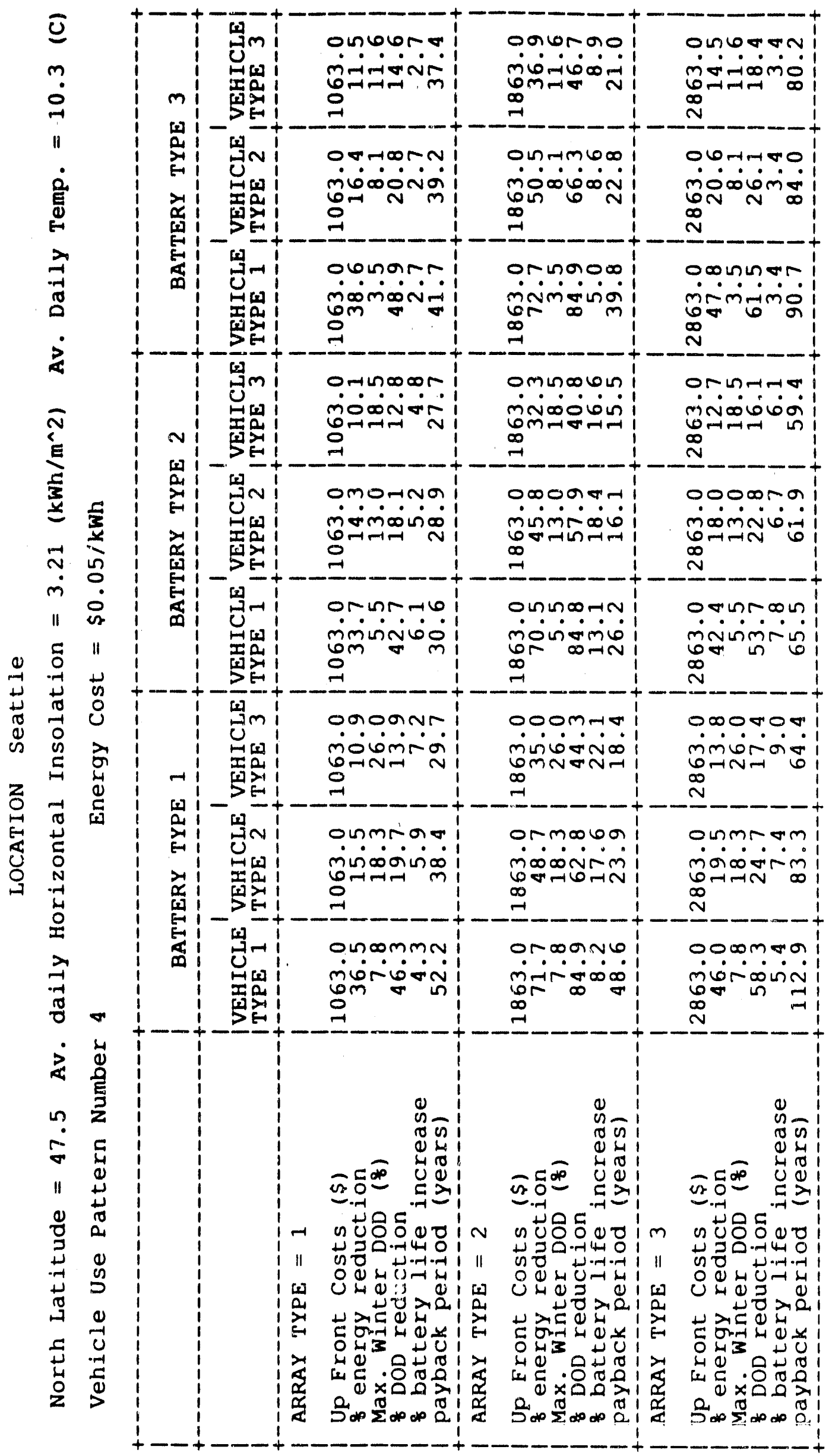




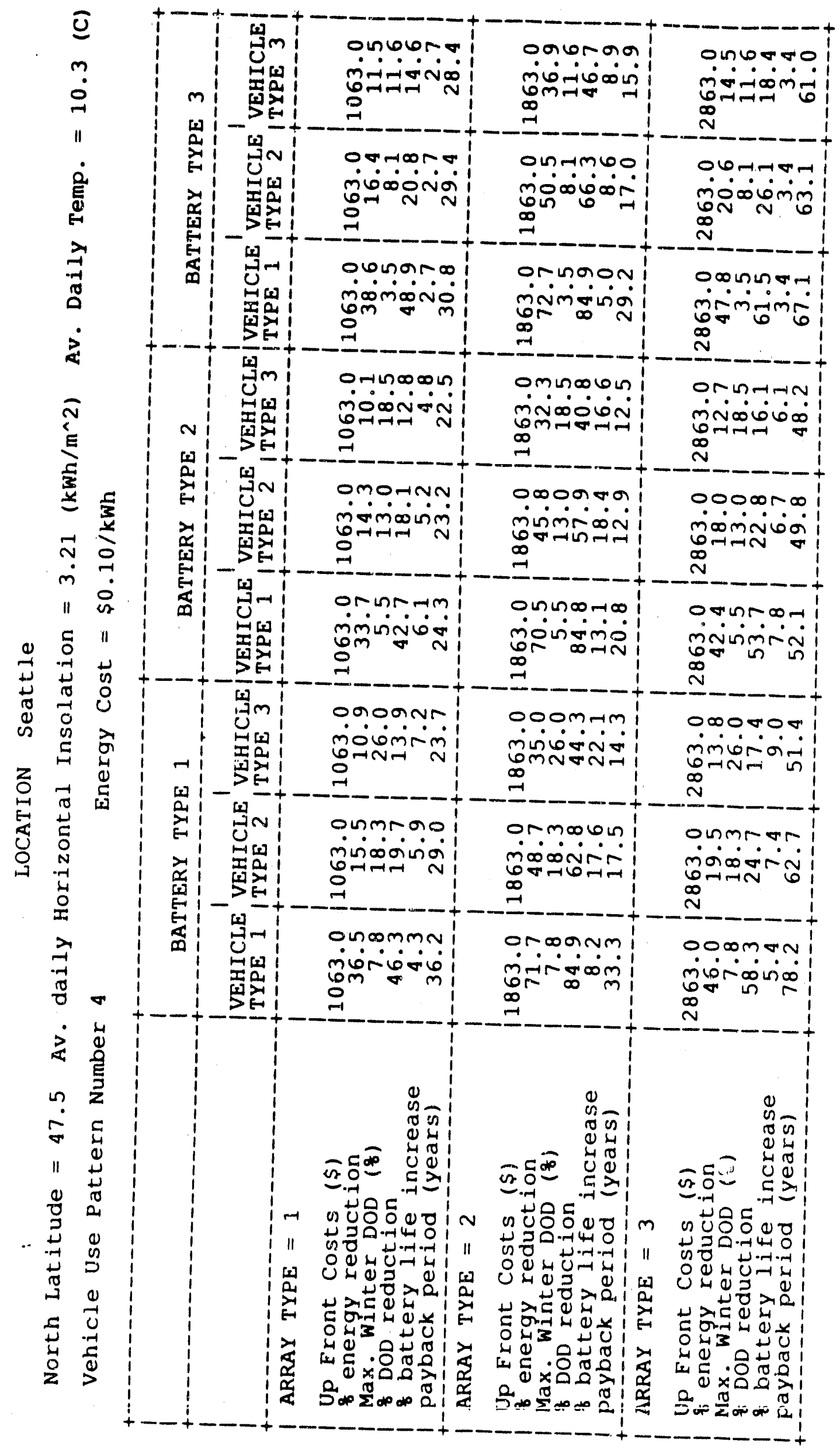

๙ู 


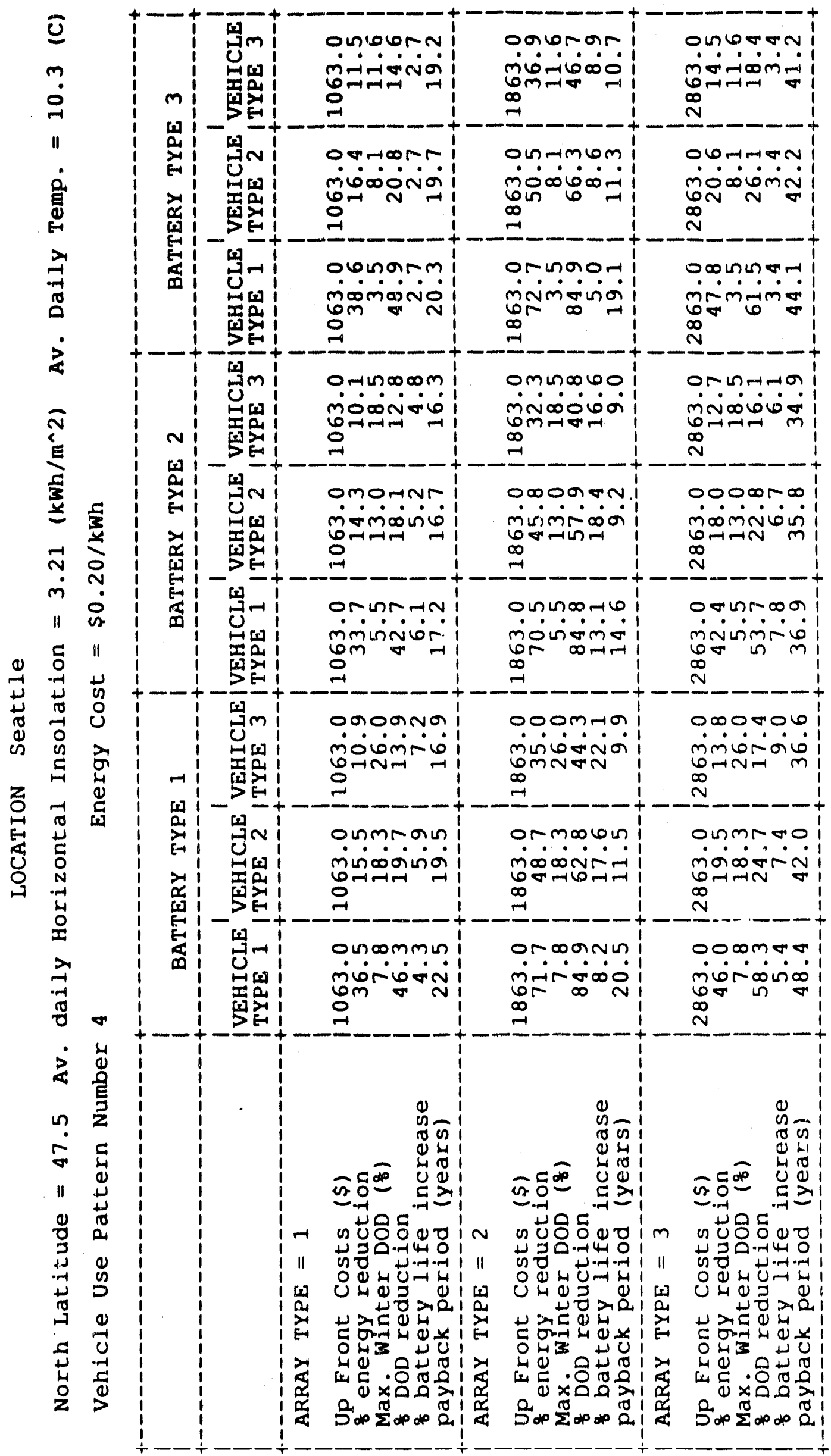




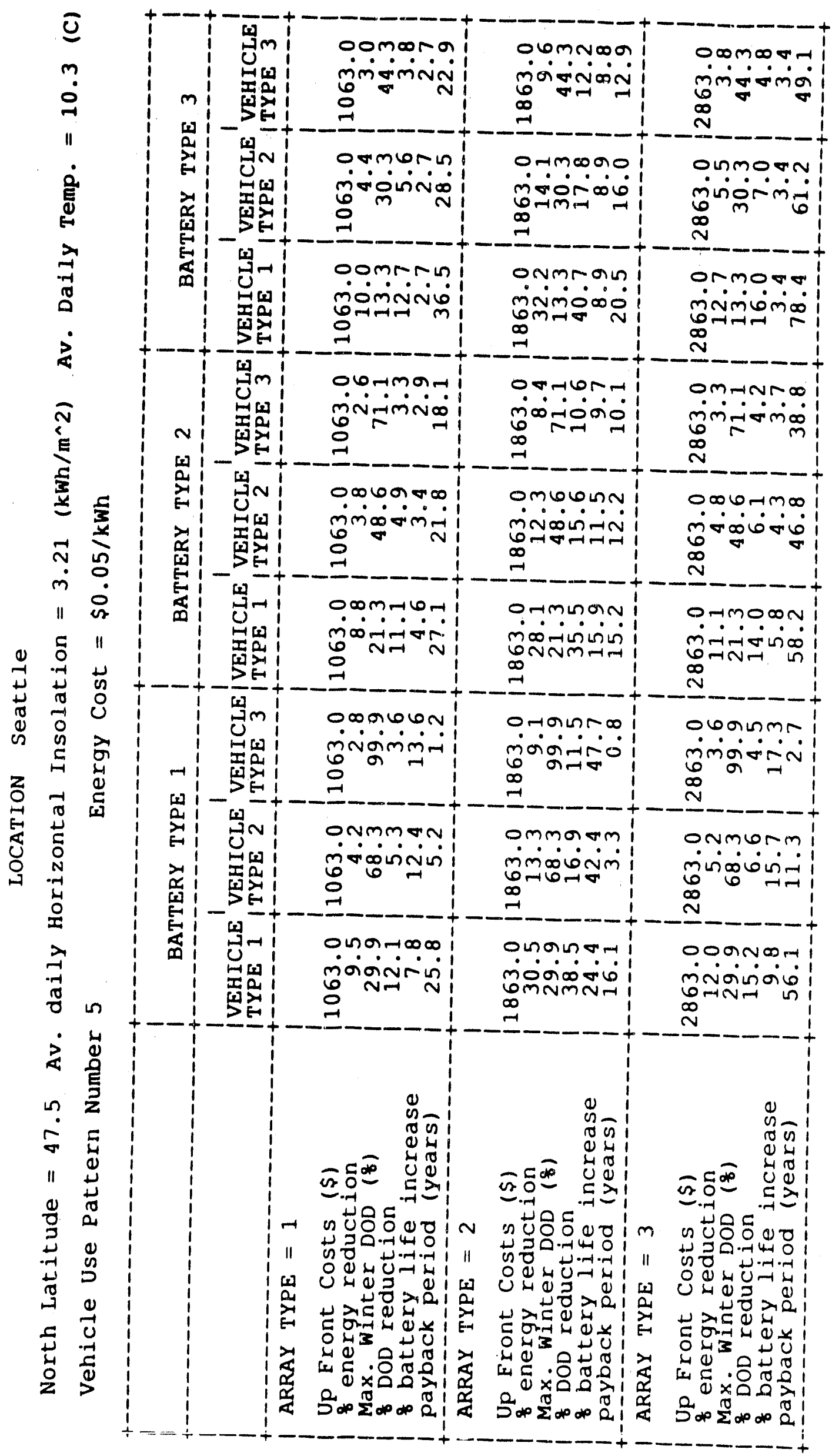




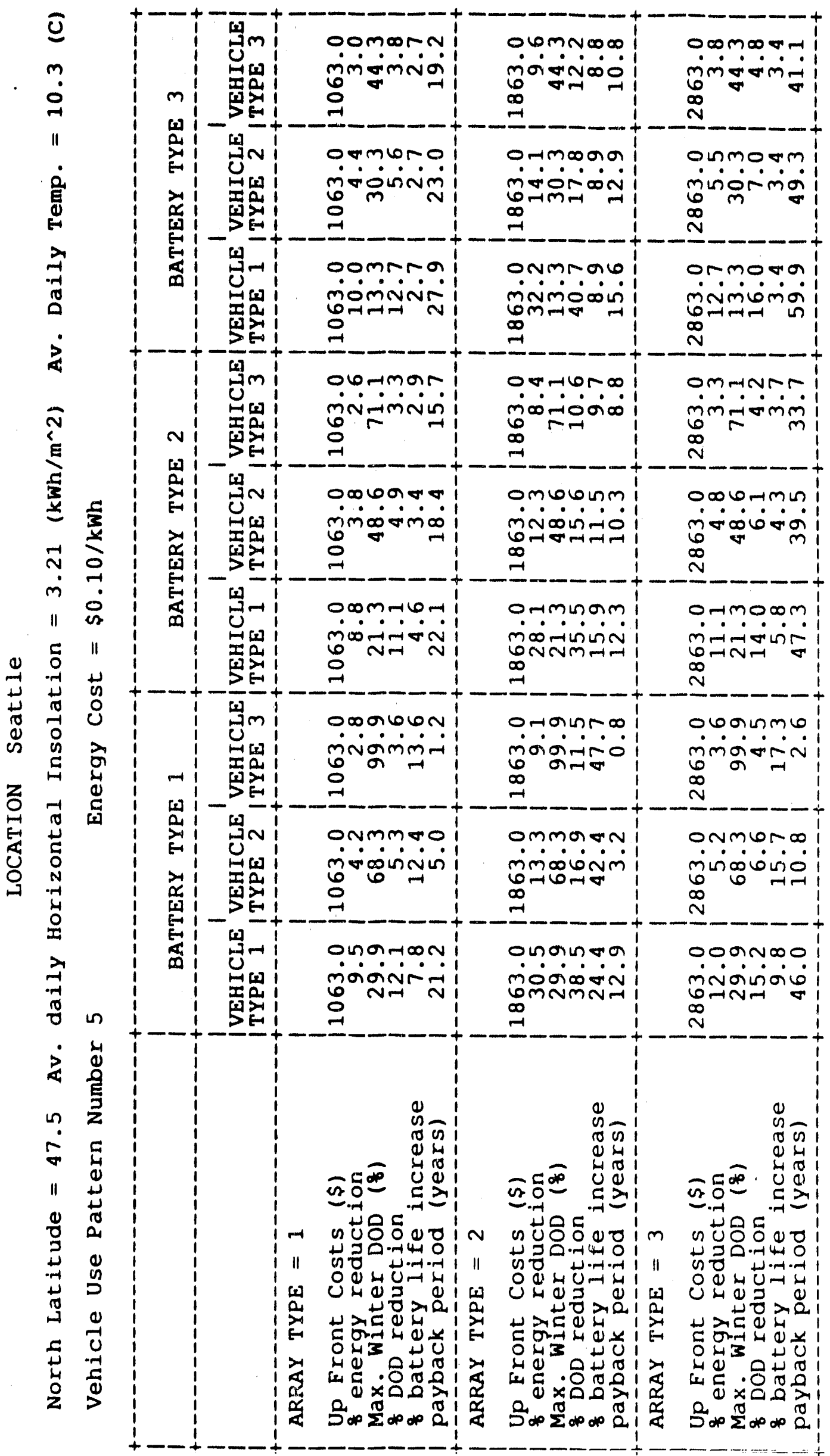




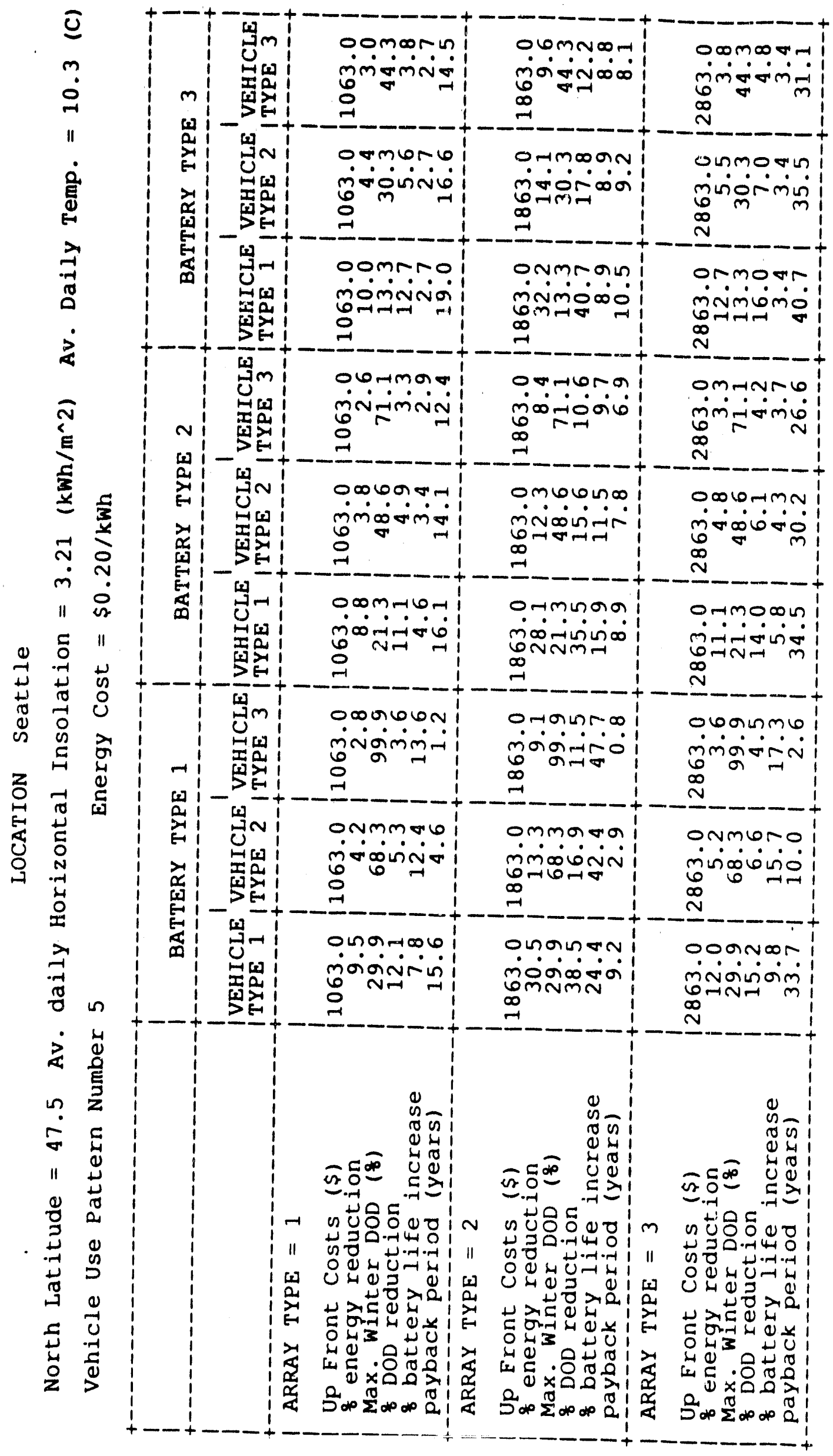




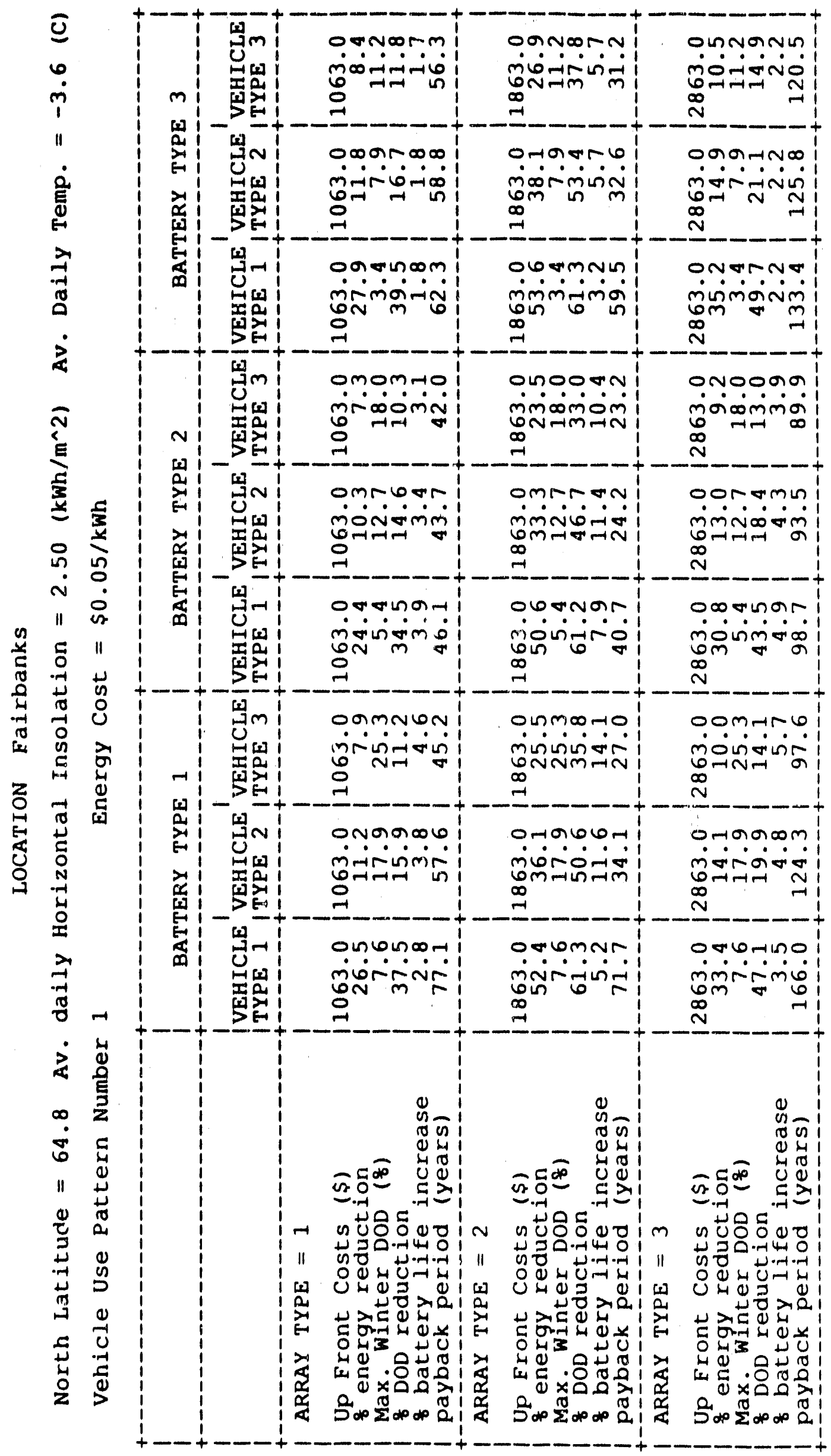

1
0
$\frac{1}{4}$
1 


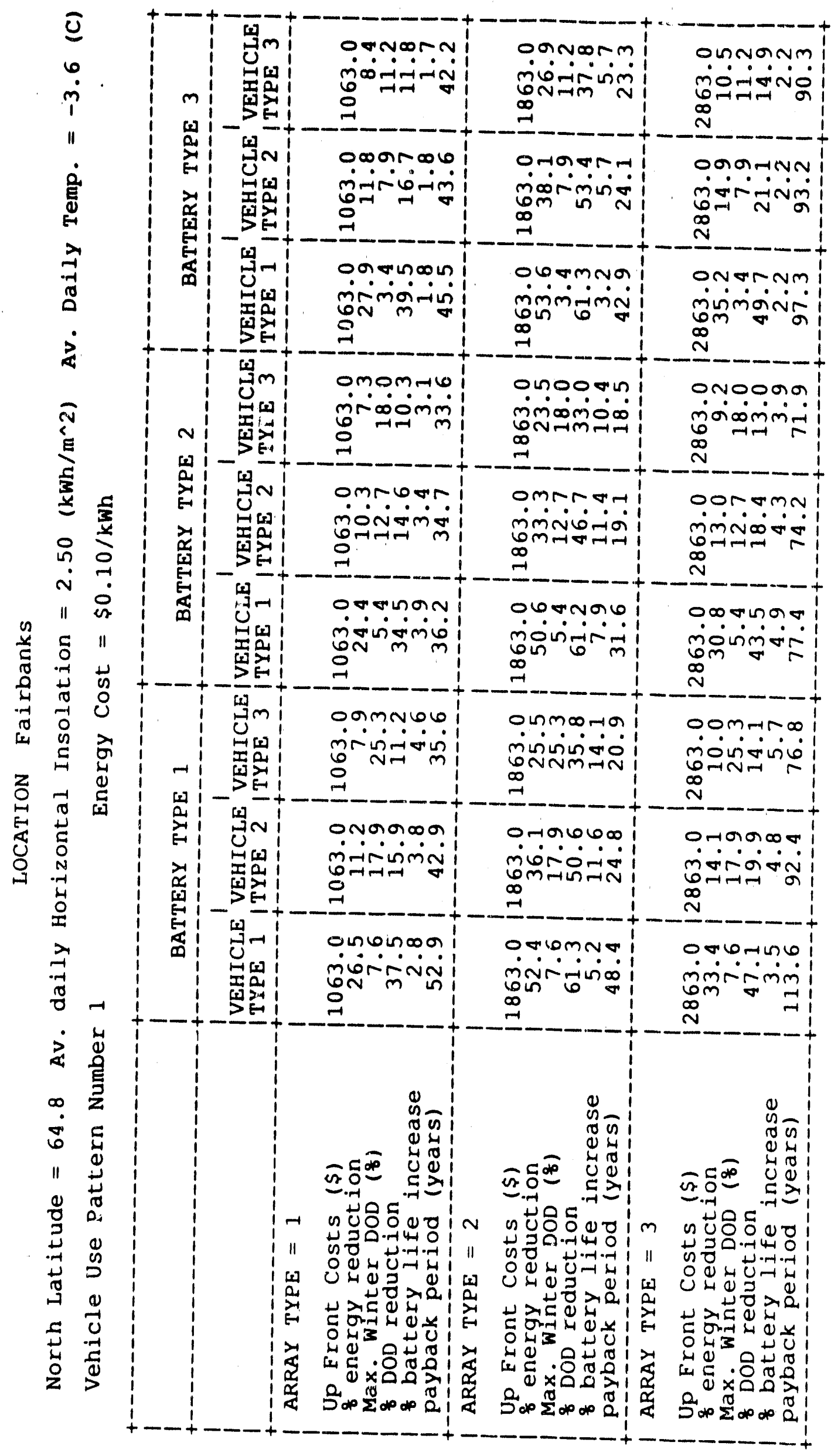

1
0
0
-1
1 


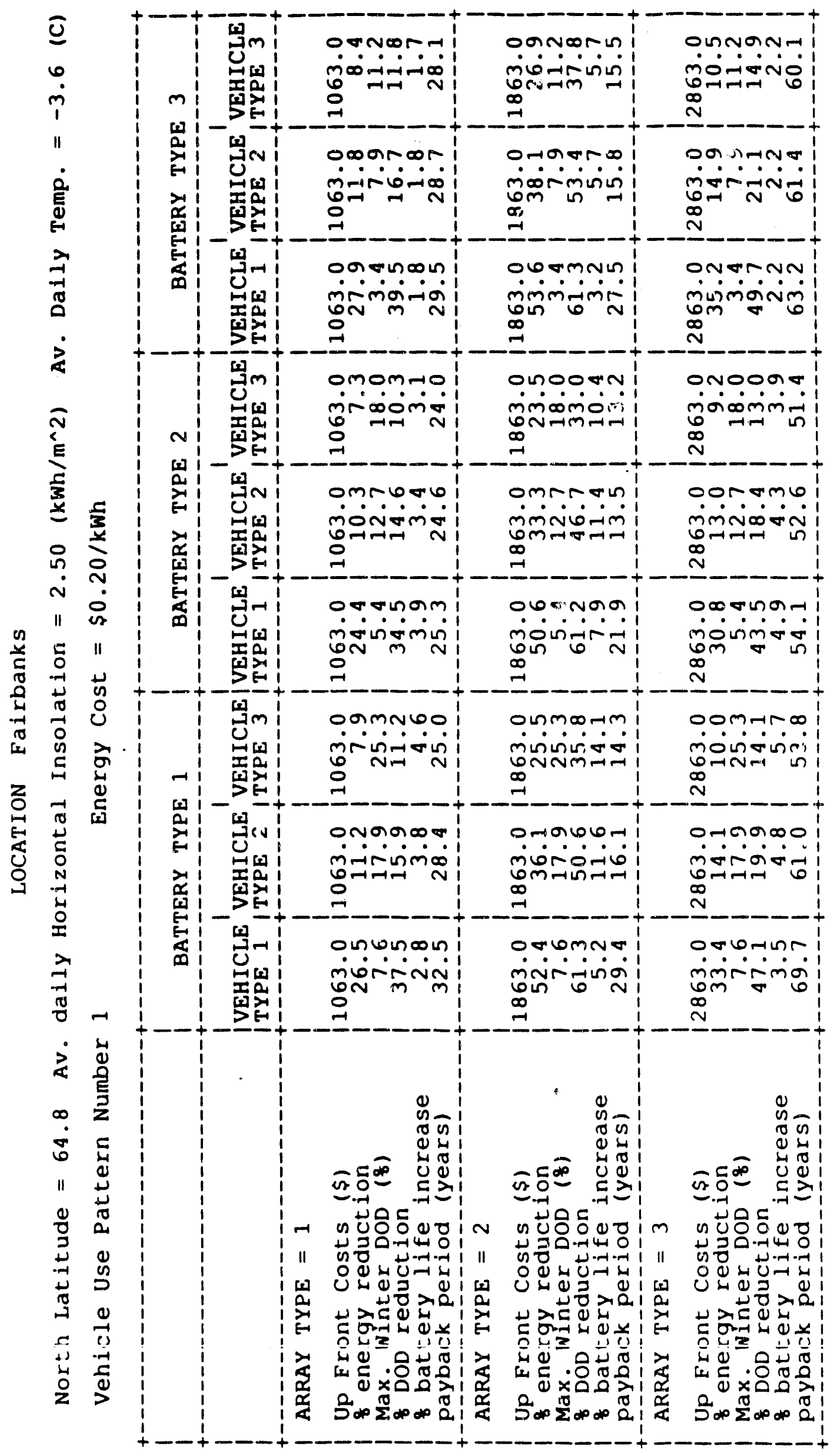




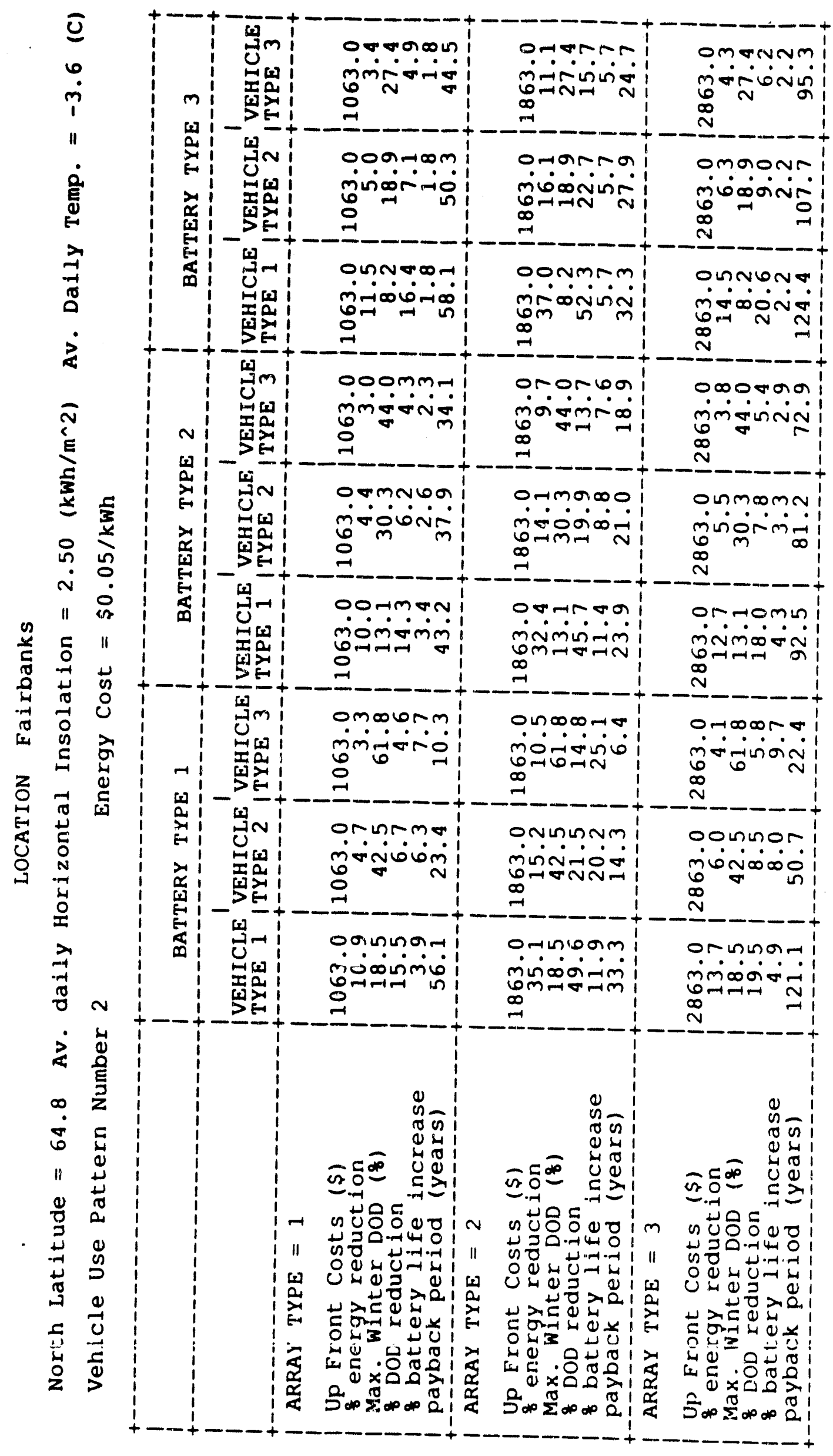




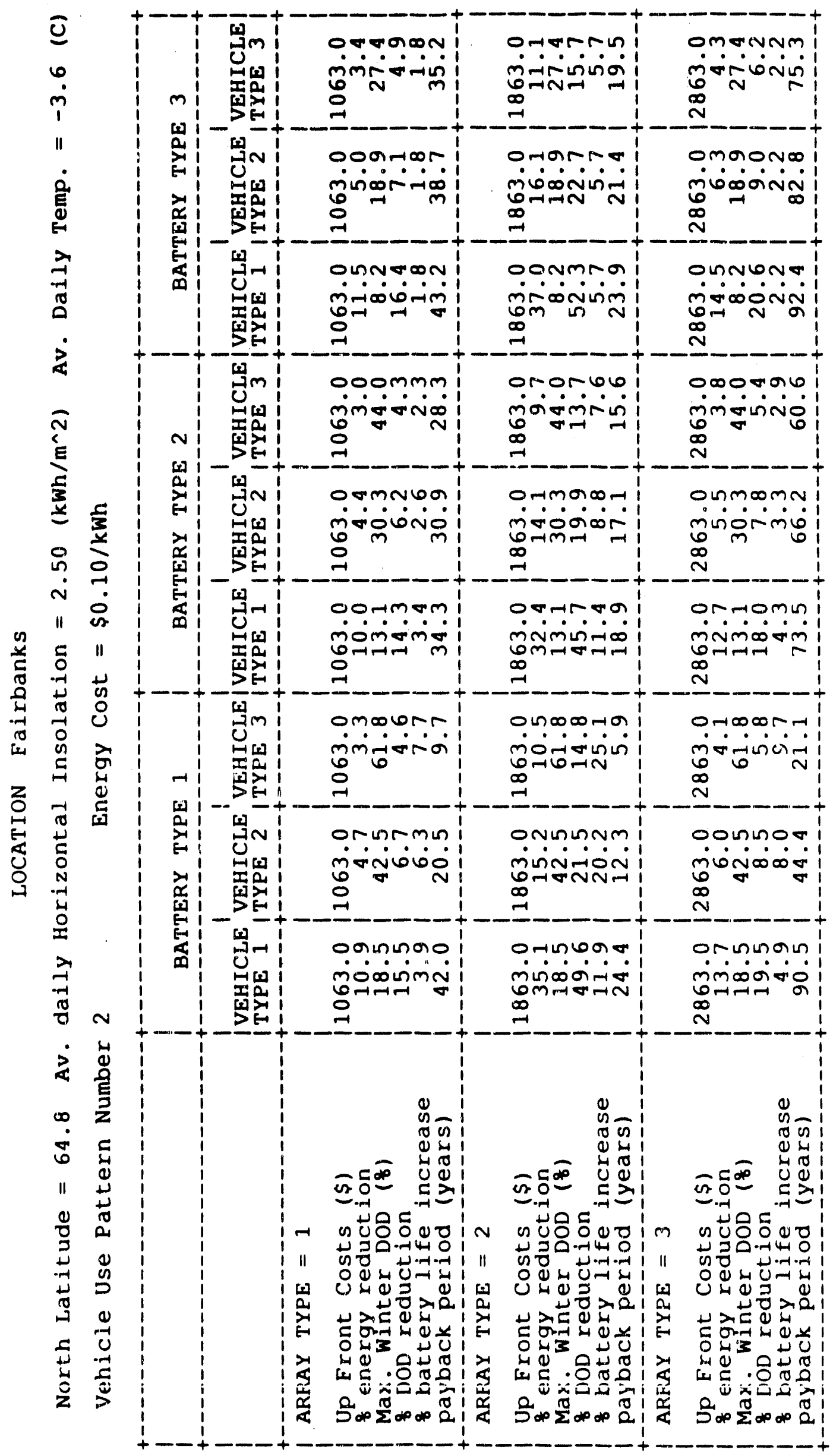




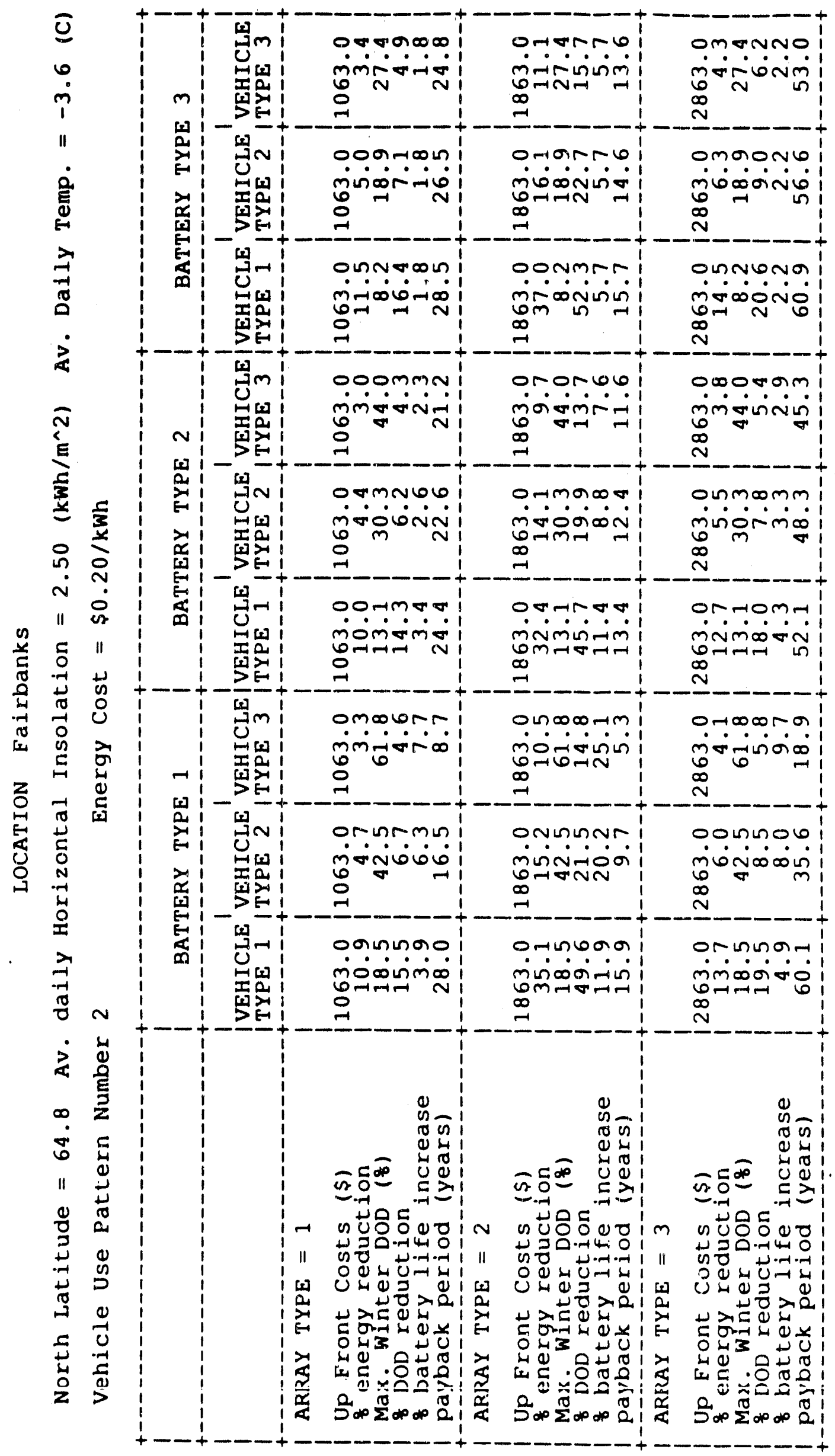




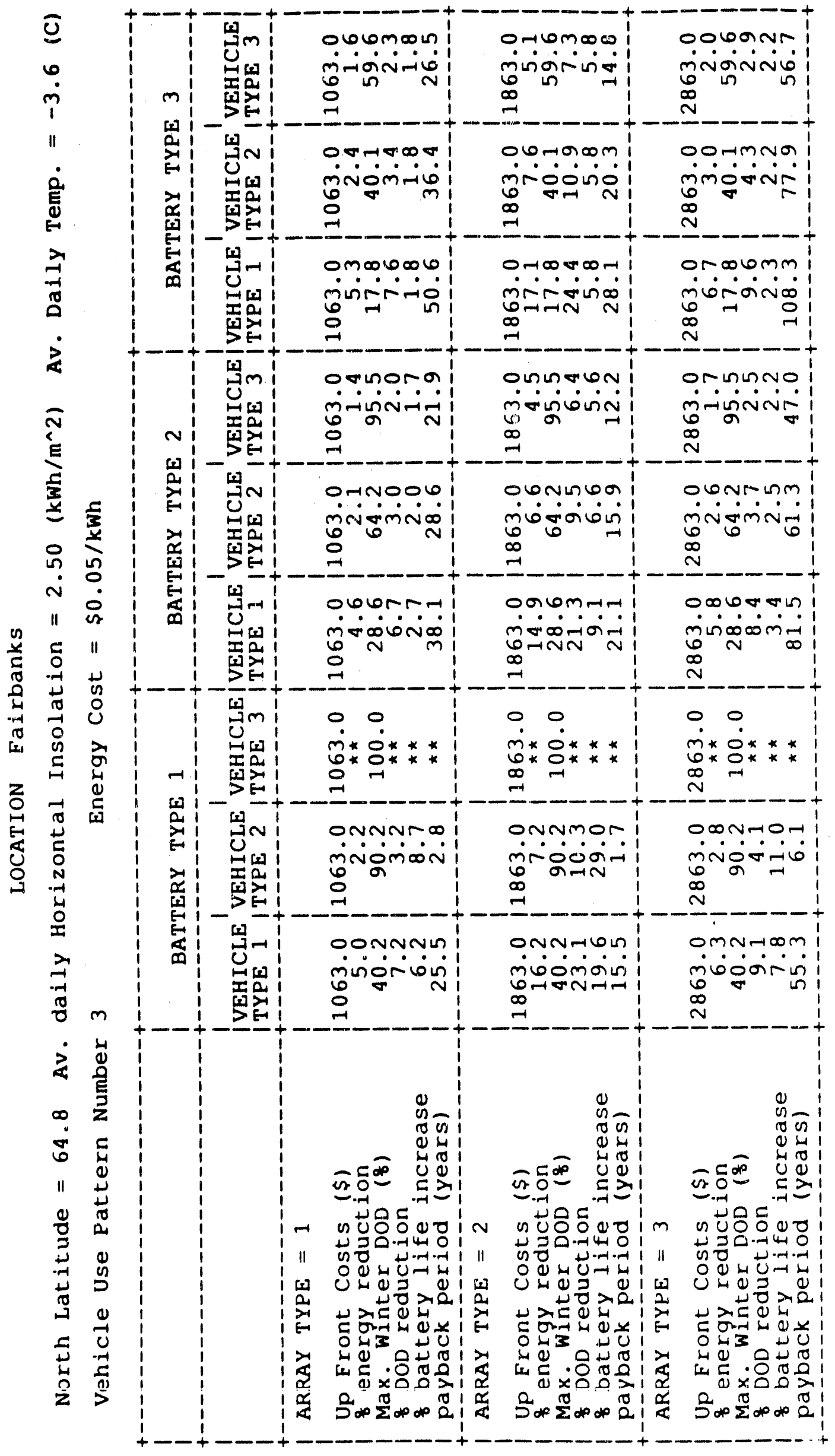




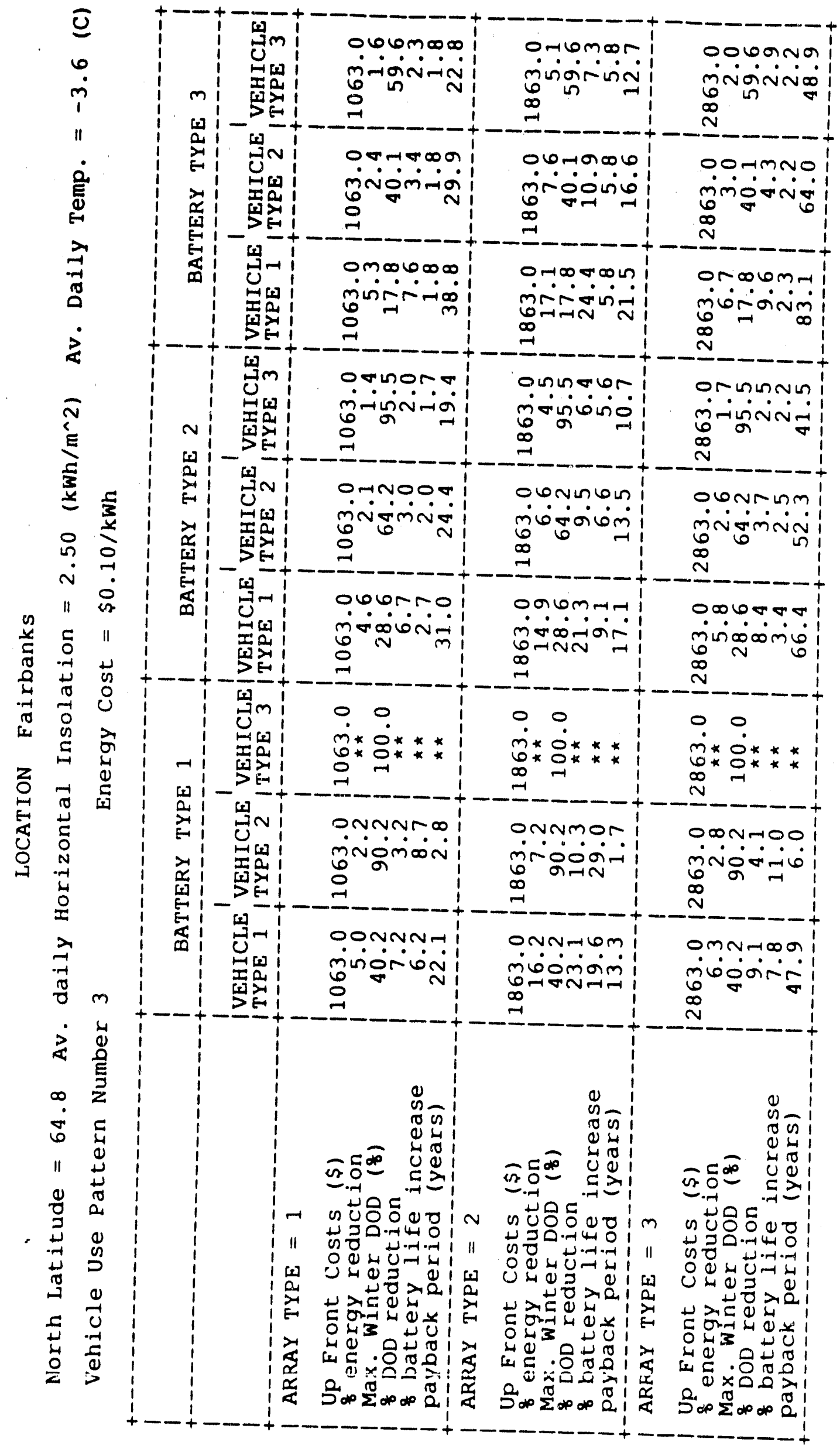




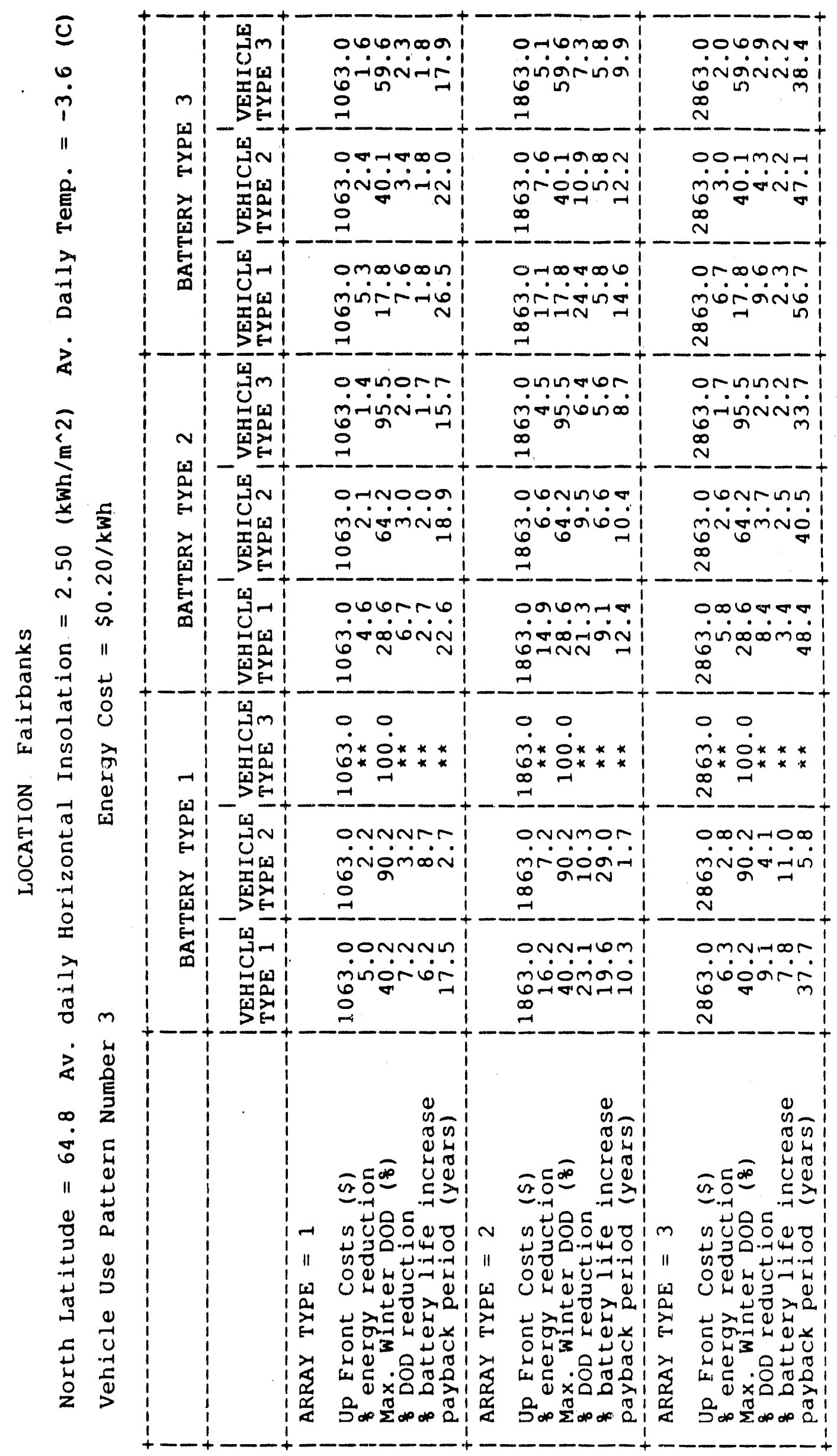




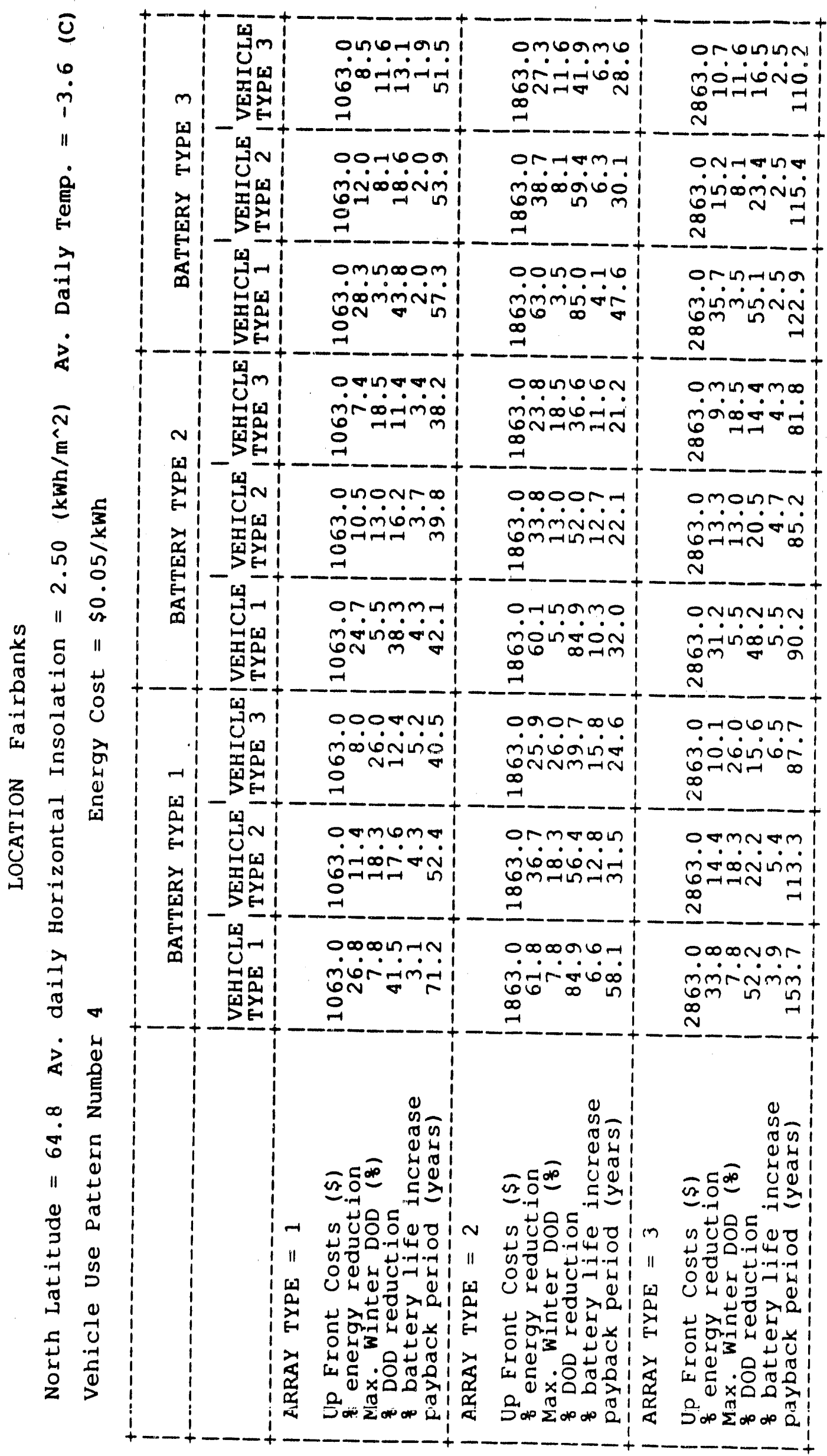




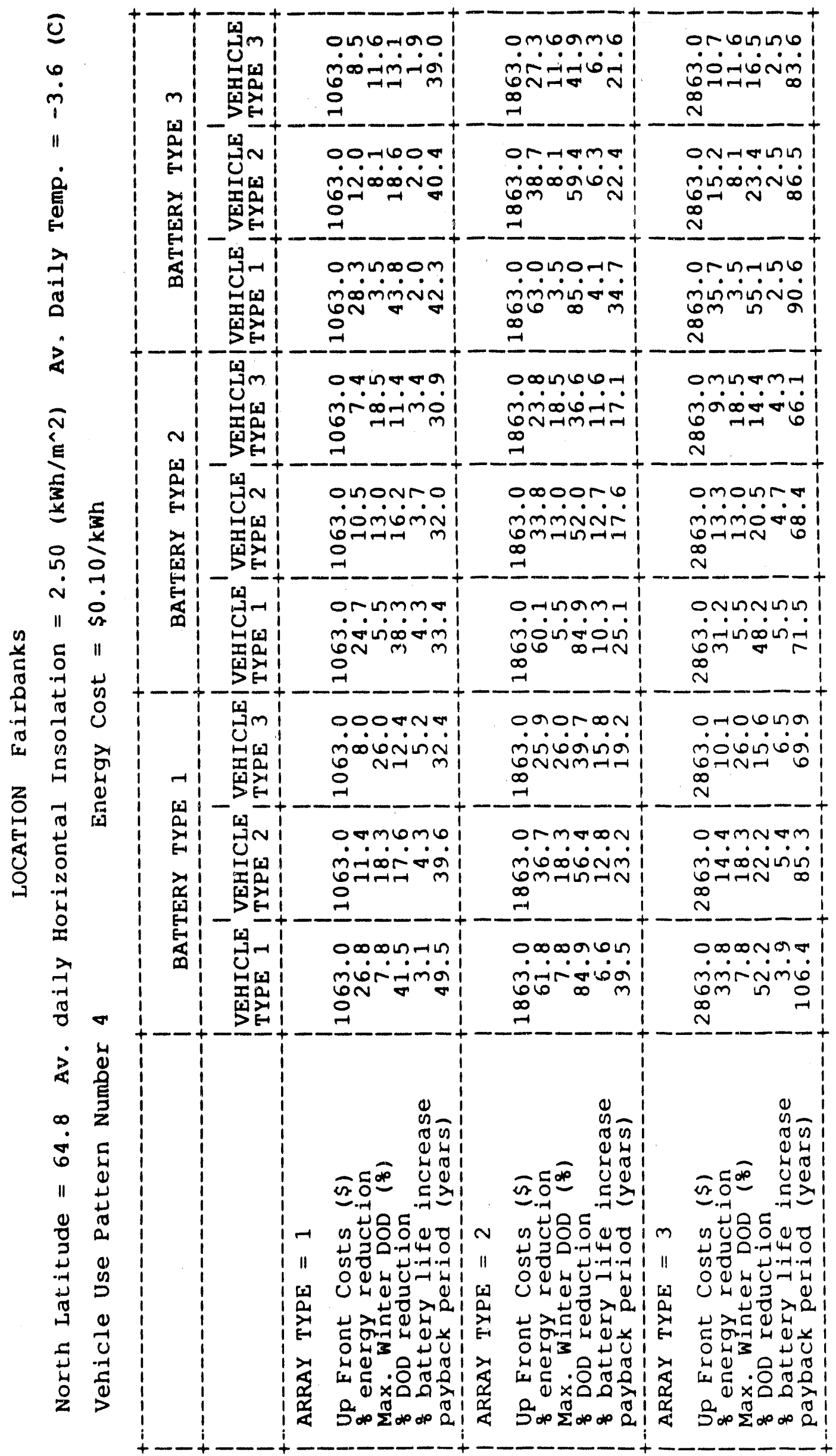




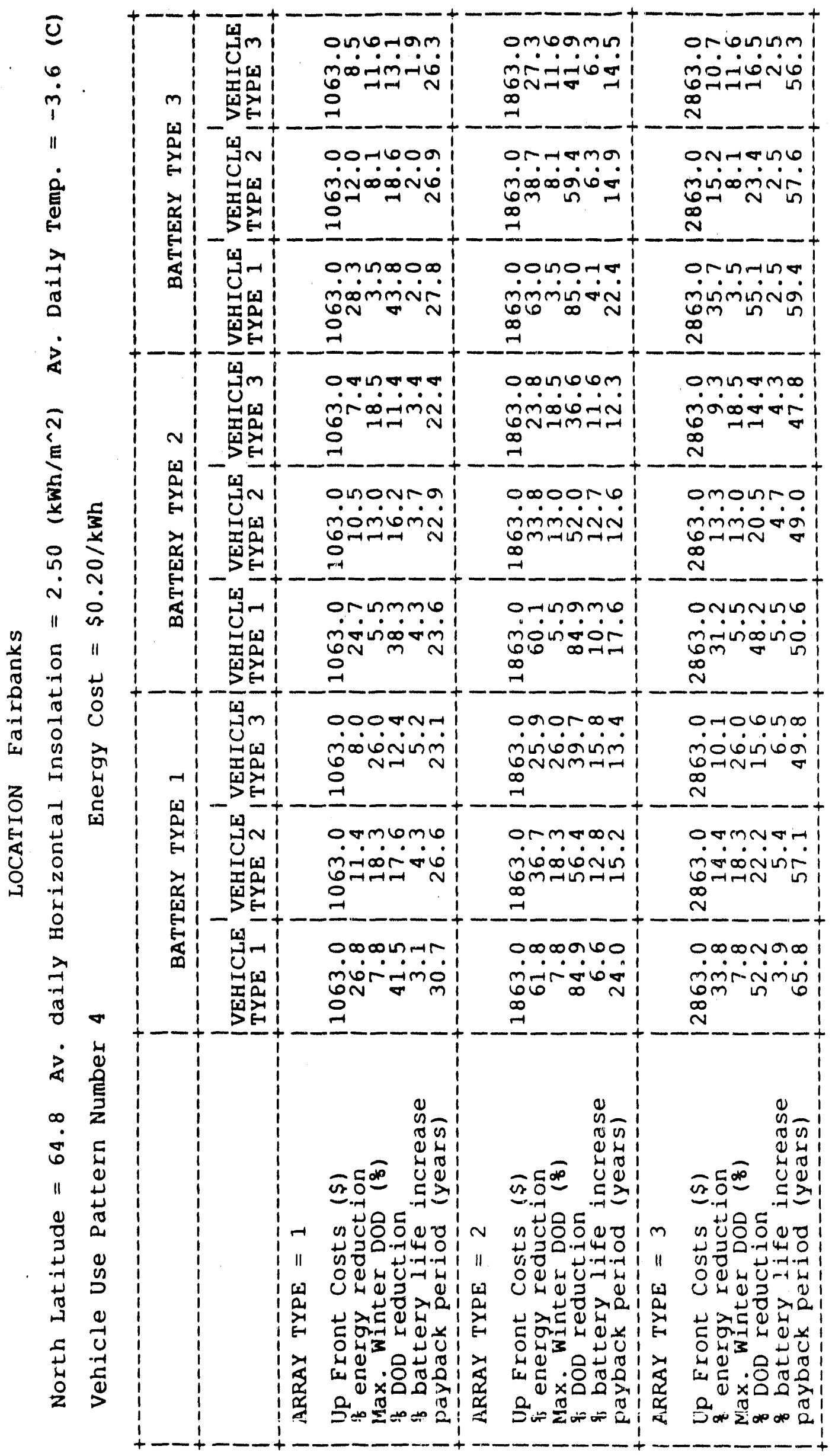




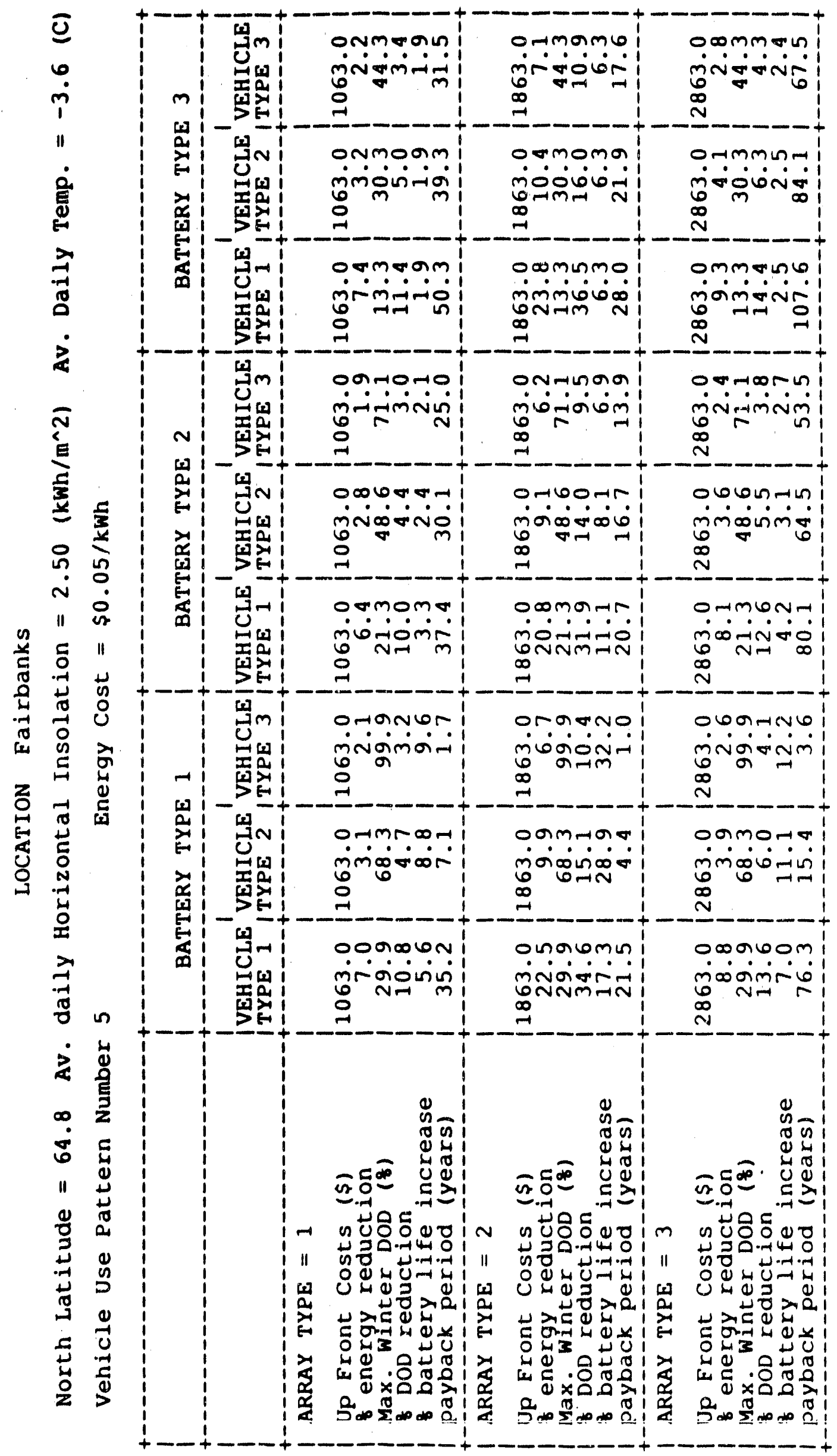




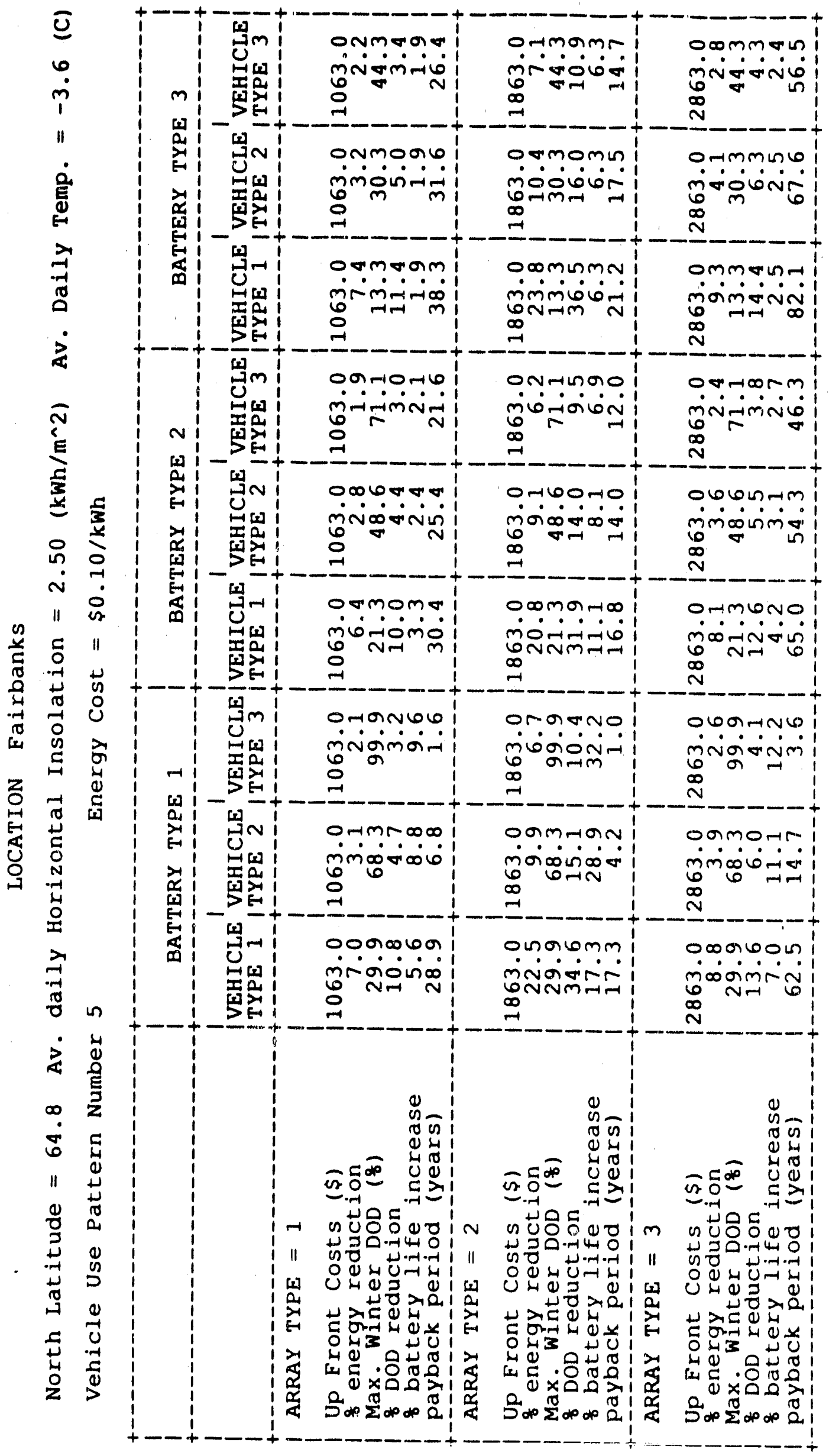




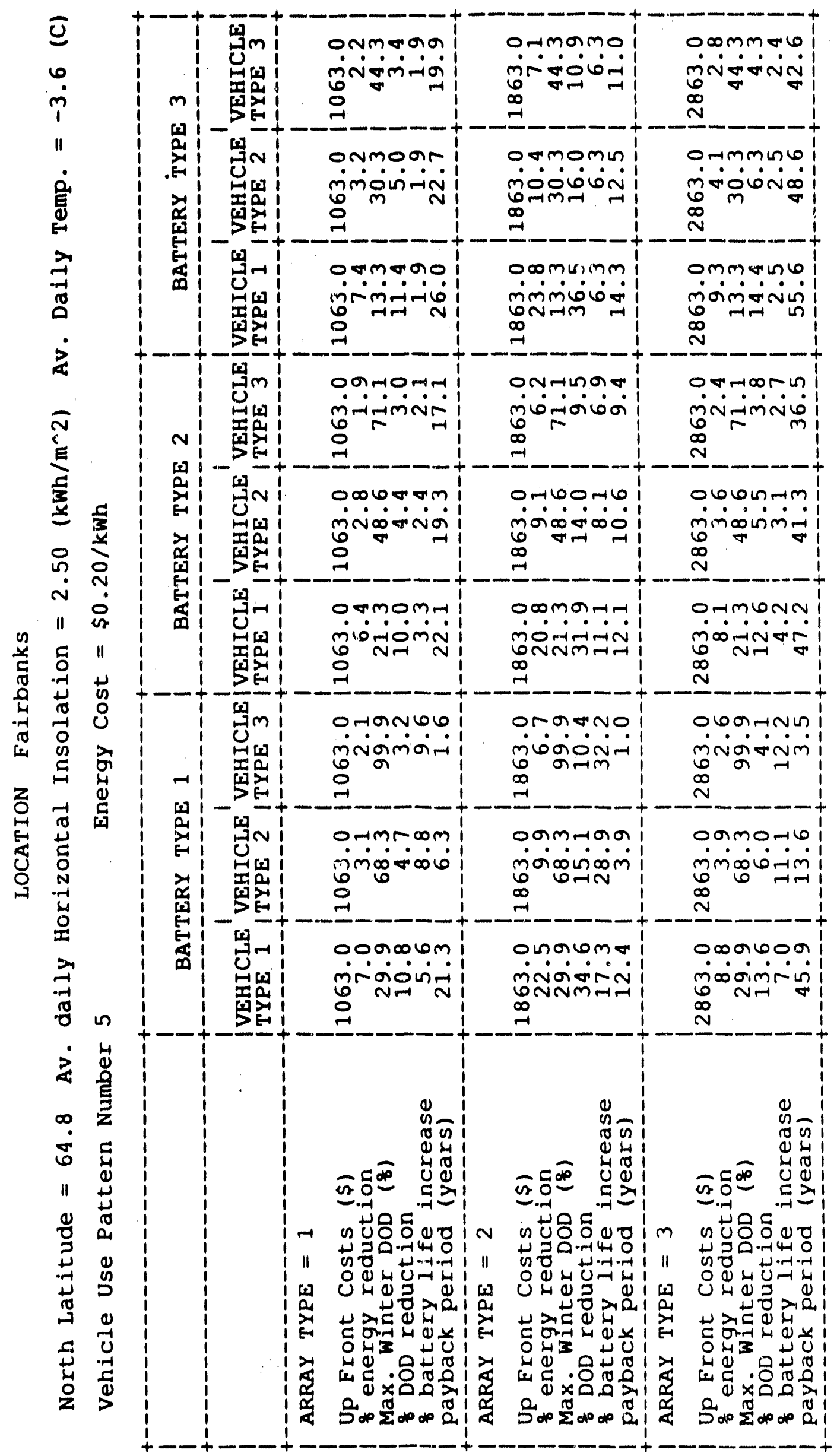




\author{
Appendix B \\ Listing of Computer Program \\ (C Language, Format TAR)
}

Note: A DC 600A Data Cartridge Tape of this program is available. This tape also contains data generated by the program which is printed in Appendix $A$. 


\section{tifndef SOIAR}

define SOLAR

/

$1 *$

Solar Array Parameters

jouble

nl,

1* array efficiency

$\star 1$

cl, / installation costr of solor array (\$)

c2, $\quad$ / cost per square meter of solar array

area, /* area of solar array (square meters)

$\star 1$

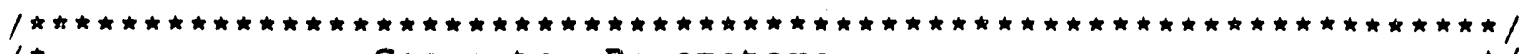

$1 * \quad$ Converter Parameters

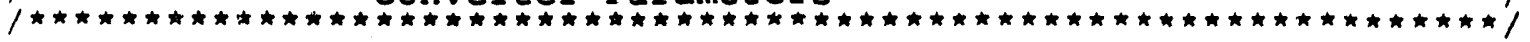

$\begin{array}{lll}\text { po, } & / \star \text { tane loss of converter (watts) } & \star / \\ \text { n2, } & / \star \text { eff. of converter converter }(\$) & \star / \\ \text { c3, } & / \star \text { cost intercept of convis }(5 / \mathrm{m} / \mathrm{m}) & \star /\end{array}$

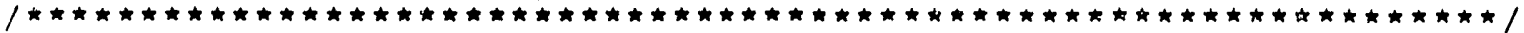

/* Vehicle Parameters

$\begin{array}{ll}\text { e0, } & \text { /* vechicle energy intercept }(\mathrm{Wh} / \mathrm{km}) \\ a^{\prime} & / \star \text { linear coefficient in model }(\mathrm{Wh} / \mathrm{km}) \star \star 2 \\ b^{\prime} & / \star \text { quartratic coefficient in model }(\mathrm{Wh}, \mathrm{km}) \star * 3\end{array}$

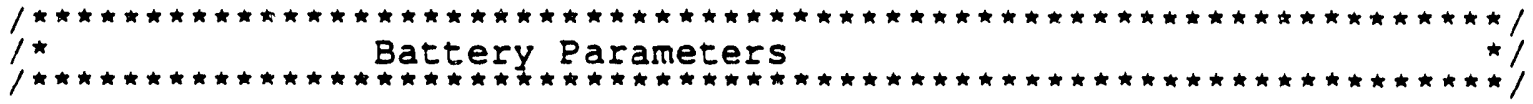

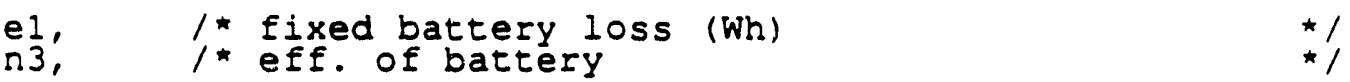

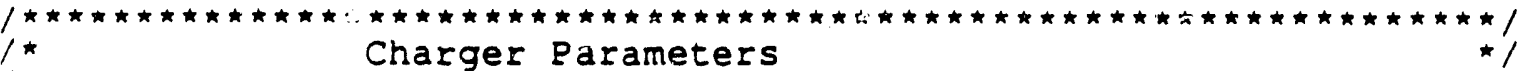

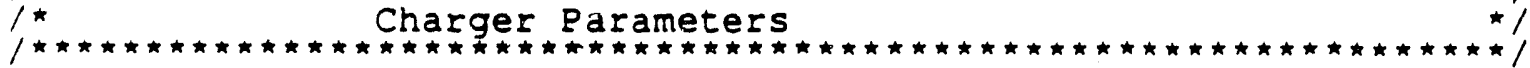

n4; $\quad 1$ * charger eff.

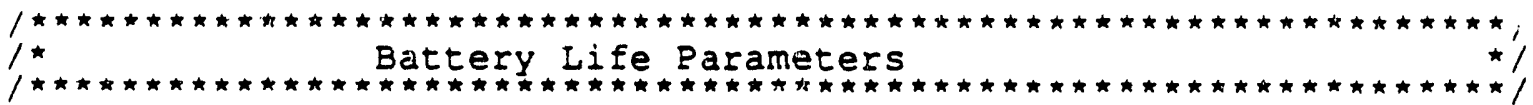

double c5, /* replacement cost of battery (includes rebate) (\$/kWh) */

e2, 1 * battery energy rating (kWh) */

NfC, $\quad$ * charge cycle life $\$$,

Ncy, $\quad / *$ dod life cycle $* /$

alpha, 1 dod sensitivity constant $* /$
$\times 0:$

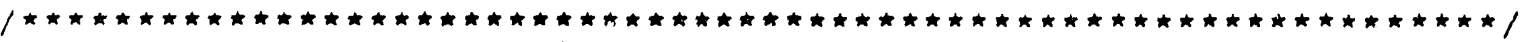

1* Electricity Cost Parameters

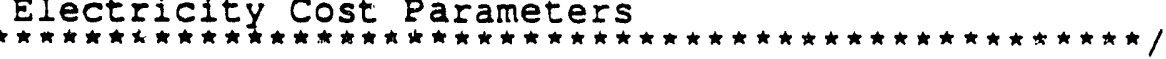

$\star /$

double c6; $\quad / *$ electricity cost $(\$ / \mathrm{kWh}) * /$

\#endif 
- ifndef global solar

define GLOBAL-SOLAR

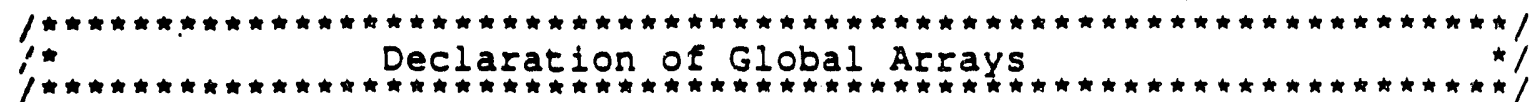

/

double

P_out $[50000]$, p-in[50000]

* power out of battery system $v[20][2]$,

coffl[4], $\operatorname{coff} 2[4]$ :

/* insolation coefficents

$\star /$

double es, ew, esp, ef, 1, latitude, dtemp;

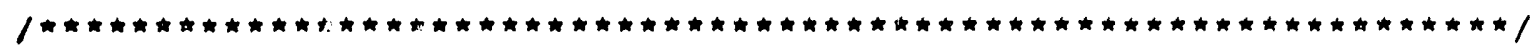

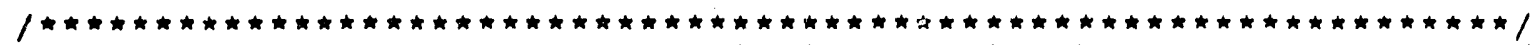

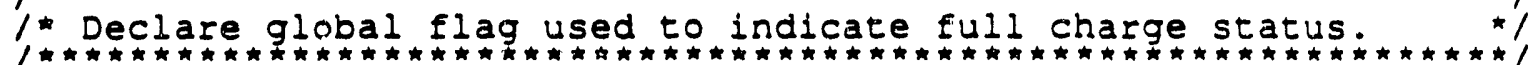

int full charge, current_season, out_of_gas [3] [3];

char location [20]:

int v_type,b_type, a_type, e_type,s_type; 
include "global solar.h"

include "solar.ñ"

include <stdio.h>

define NO SEASONS

4

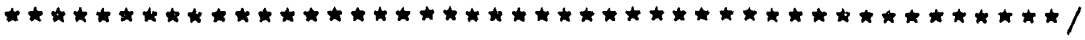

/* This program was developed to decermine the feasibility of

/* placing solar arrays on electric vehicles.

/* The simulation will be performed for a total of:

/* 8 locations ( 1 page of data for each season),

/* 5 vehicle speed models,

/* and 3 battery types,

$1 *$ and 3 vechiles.

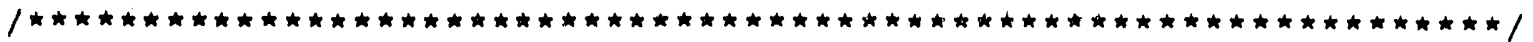

$\operatorname{main}()$

1

/* declare external functions */
extern double
extern double
extern double
extern double
$S(), V()$;
battery dep season ():
model PV system ():
extern double
model-charger ():
model_vehicle_energy_use () :

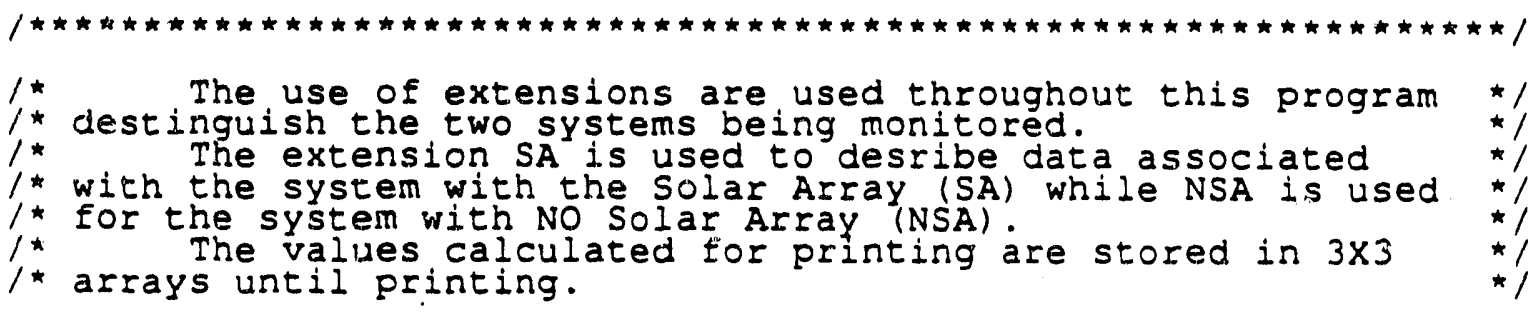

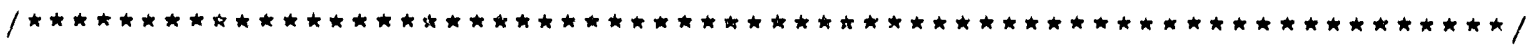

double ENSA,ESA, electricity cost SA, electricity cost NSA;

double season cost SA[4], season Cost_NSA[4], Iife_SA[4], Iife NSA[4];

double life year SA, life year NSA;

double daysT4], sēason cost yeār_SA, season cost year NSA;

double cost saved[3] [3], baEtery-cost SA, bāterȳ CoSE NSA, payback [3] (3];

double dod SA, dod NSA, maxdod SA,maxdod NSA, big;

double speed, inpuE, pos engy, ñeg engy, EIN;

double percent energy reductiont3] [3] percent dod reduction [3] [3];

double percent battery life_increase[3] [3]:

Jouble AESA[4],AENSA[4T;

double cost SA[3][3], cost NSA[3] [3], upfrontcost [3] [3];

double maxdodyear_SÁ[3] [3], maxdod_year_NSÁ[3][3];

float time, dE:

int $i, j$, upper, $1, m$;

char stringl[120\}, string2[10];

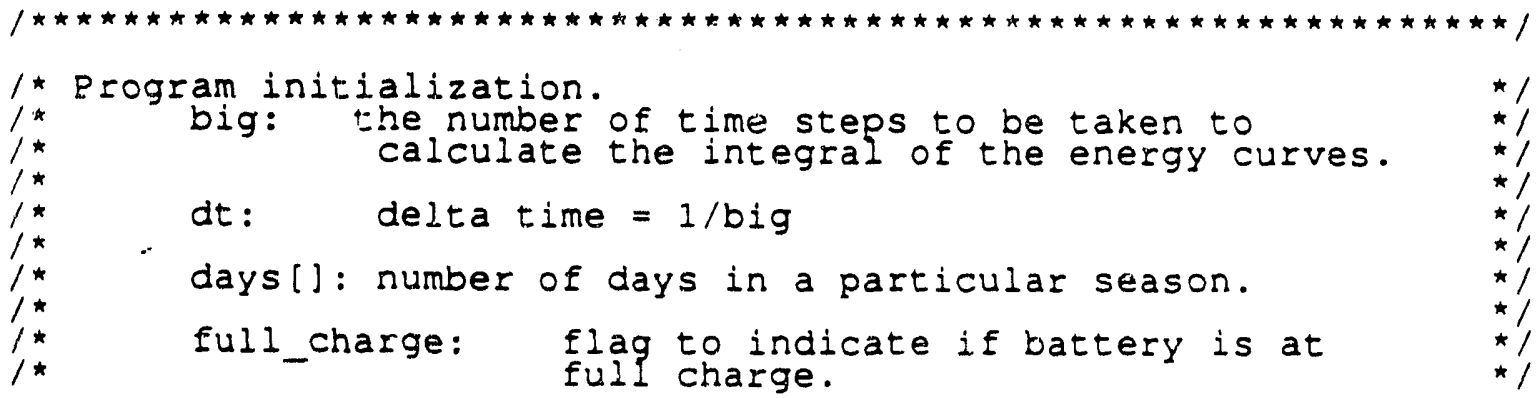

B-4 printed 07/25/90 3:20 PM 
setbuf (stdout, NULL) :

days $[0]=$ (double) $91.0 ;$
days $\{1]=$ (double) $92.0 ;$
days $[2]=$ (double) $91.0 ;$
days $[3]=$ (double) $91.0 ;$

big $=$ (double) 100.0 ;

$d t=($ float $)(1 / b i g)$;

$\operatorname{maxdod} S A=($ double $), 0.0$;

$\operatorname{maxdod}$ NSA $=$ (double) 0.0

full_ch̄arge $=1$;

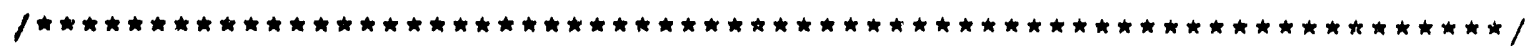

1. Initialize the data files for parameters

$\star /$

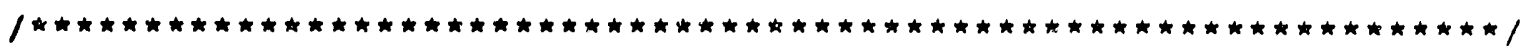

read $S(1)$ :

read-convert (1):

read-charger (I):

upper $=($ int $)(24 / d t)$;

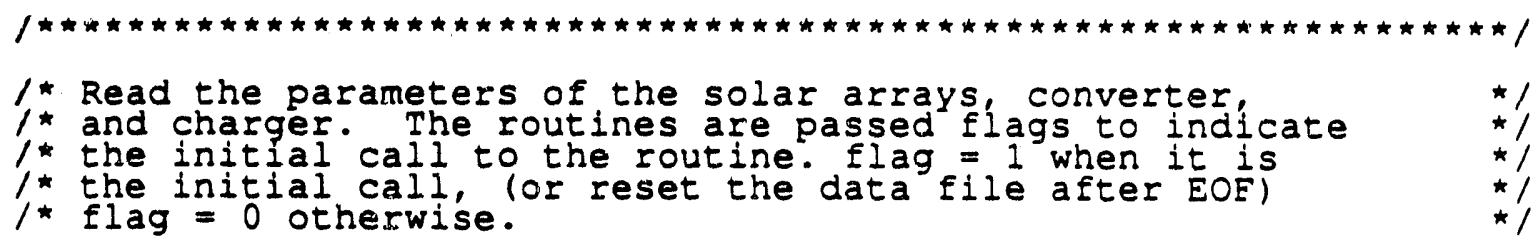

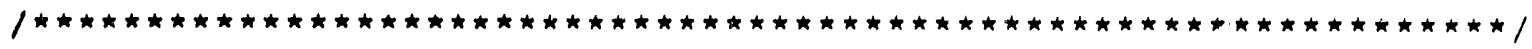

read convert $(0)$;

read-charger $(0)$;

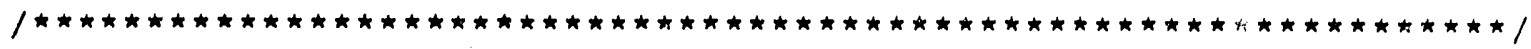

/* Read the values for the insolation model and the vehicle */

/ speed model. These values are placed in external variables */

/ for easy access. The values of $\mathrm{s}$ read in are in units of /

* Watts $/ \mathrm{m}^{*} \star 2$ so the area of the solar panels, read in by ,

/* read array ()' is mutiplied to this to give units of watts. $* /$

while (read $s(0))($

while (read $\nabla(0))^{\prime}$ l

read cost (1):

ead cost (0)) i

print_head ();

while (read array ( $)$ (1)) ;

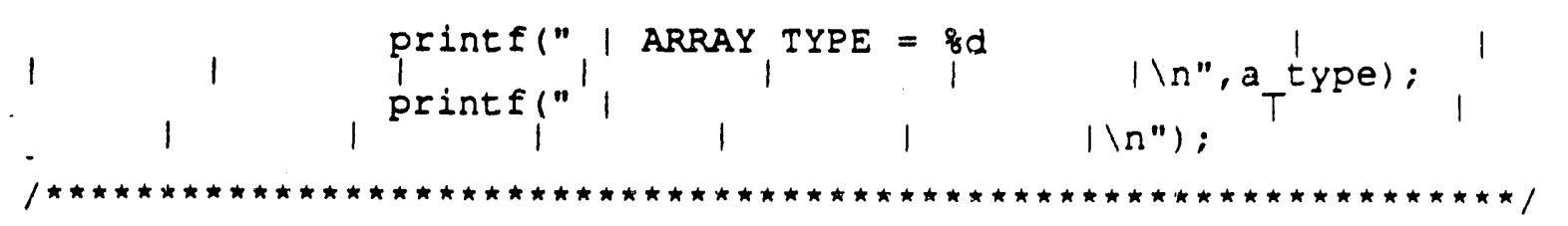

1* Read the vehicle, battery, and battery life parameters. */ 


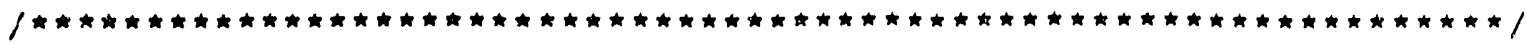

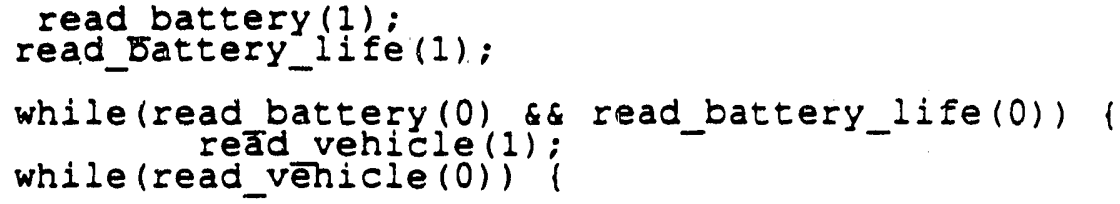

$1^{*}$ This is the main loop of the procedure. The power curves */

/ for the propulsion system are generated with and without * *

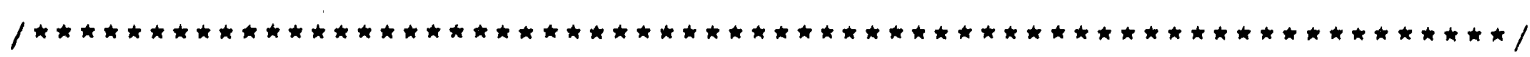

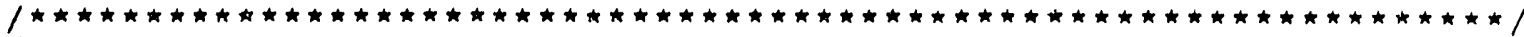

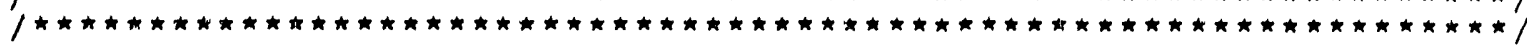

out of gas [v type-1] [b type-i] = 0 ;

maxdod_year_NSA [v_type=i\}[b_type-1]=0.0;

for (current season=0; current_season<NO_SEASONS; current_season ++ ) 1

AESA [current season] $=0.0$ :

AENSA [CurrenE season] $=0.0$;

$\mathrm{V}(0.0,1) ;$

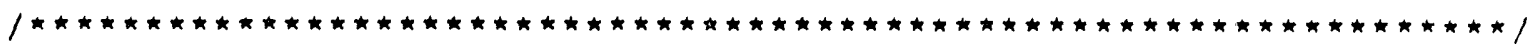

/ Initialize positive and negative energy counters and others. */

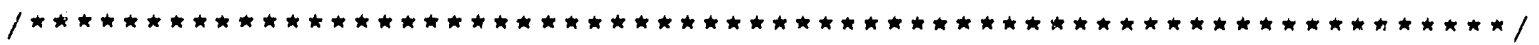

full charge $=1$

pos engy $=$ (double) 0.0 ;

neg-engy = (double) 0.0 ;

$E I N=$ (double) 0.0 :

$E S A=$ (double) $0.0:$

$E N S A=$ (double) 0.0 ;

maxdod $S A=$ (doubie) 0.0 ;

maxdod $I N S A=$ (double) 0.0 ;

time $=0.005$ :

for $(i=0 ; i<=u p p e r ; i++)$ (

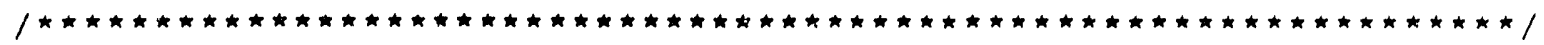

/ Calculate the power output from the battery system with

/* and without the effects of the solar arrays.

P_out $[i]=$ model_vehicle_energy_use (speed, $a, b, e 0, n 3)$;

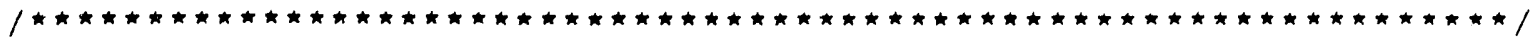




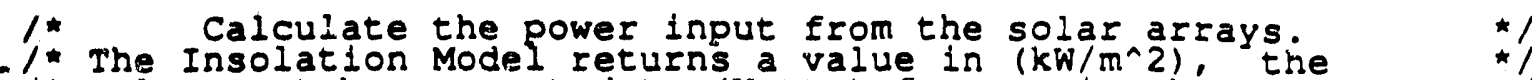

1* The Insolation Model returns a value in ( $\mathrm{kw/m} / 2$ ), the

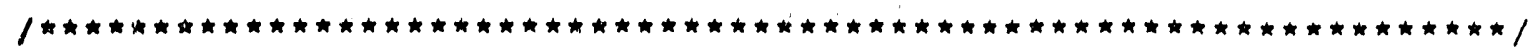

$$
\text { input }=S(t i m e, 0) \star \operatorname{area} \star 1000 ;
$$

/

1 * If the battery is at full charge the input from the solar */ 1 * arrays it equal to zero.

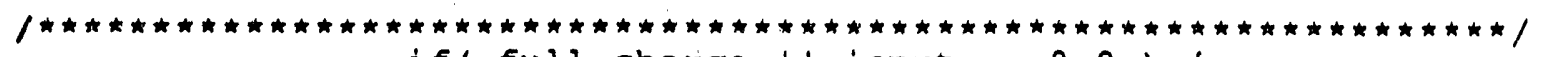

$$
\text { if ( full charge } \mid \text { l input }==0.0 \text { ) } 1
$$

1

p_in $[1 .]^{2}=$ (double) 0.0 ;

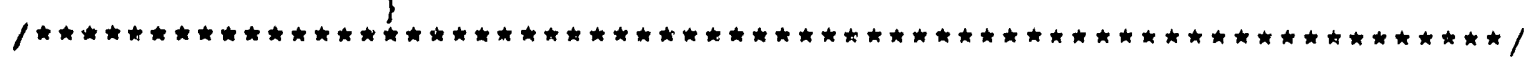

1* Else model the power input by the PVs.

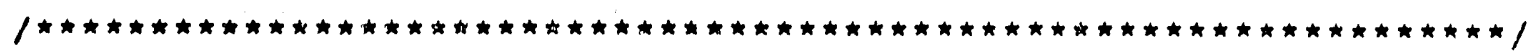

else P_in $[1]=\operatorname{model} P V \_$system $(i n p u t, n 1, p 0, n 2)$ :

P_out_SA[i] = p_out $[1]-p_{\text {_in }}[i] ;$

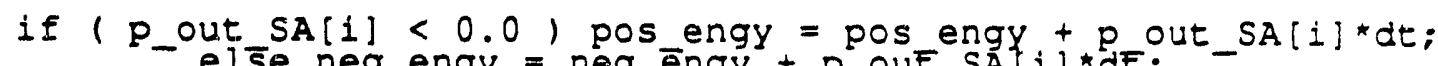
elise neg_engy $=$ neg_engy + p_ouE_SAli]*dE:

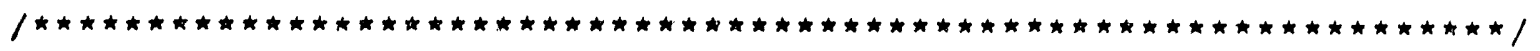

1* The amount of energy 1nput by the solar arrays can not

/* exceed the energy output by the propulsion system. If

/ the solar arrays input all the energy thus taken out,

/ the battery is at full charge and the flag set.

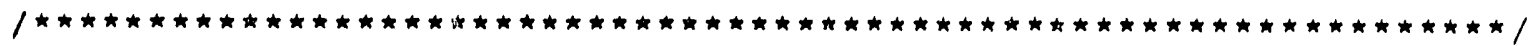

$$
\text { if ( (pos_engy + neg_engy) <0.0) full_charge = } 1 \text {; }
$$

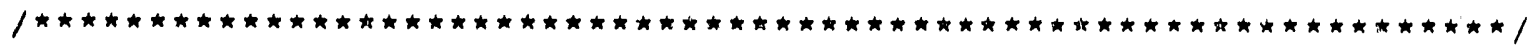

1* Calculate the integrals of the power curves to obtain the

/ energy used by the propulsion system with and without

1* the effects of the solar panels. The energy delivered by

/* the solar panels is also calculated.

$$
\begin{aligned}
& E S A=E S A+p \text { out } S A[i] \star d t ; \\
& \text { ENSA }=E N S A+\bar{p} \text { OUE[i]*dt; } \\
& E I N=E I N+P \text { - In }[1] \star d t ;
\end{aligned}
$$

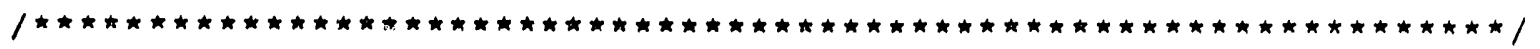

/* If the energy taken out of the battery is equal or greater

$1 *$ than the initial battery energy we have effectively

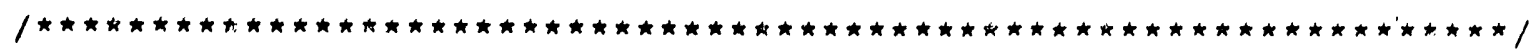

if (ENSA $>=1000 *$ 2) out_of_gas [v_type-1][b_type-1] = 1;

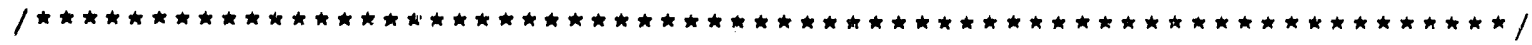

1* Calculate the depth of discharge for the battery and save

1* the maximum value to be used in the Battery Depreciation

* Model. 


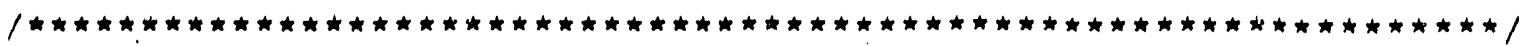

$$
\begin{aligned}
& \operatorname{dod} S A=E S A /(e 2 * 1000) ; \\
& \text { dod NSA }=E N S A /(e 2 * 1000) ; \\
& \text { if ( } \mathrm{Cod} S A>\text { maxdod } S A \text { ) maxdod } S A \text { at dod } S A \text {; }
\end{aligned}
$$

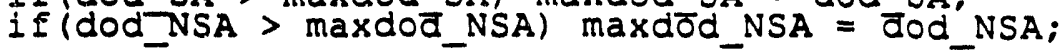

$$
\begin{aligned}
& \text { time = time }+d t ; \\
& \text { 1 } 1 \text { * end of time loop *1 }
\end{aligned}
$$

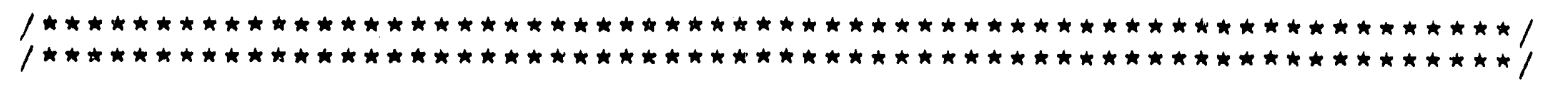

/* Store a running total of the energy used per season * *

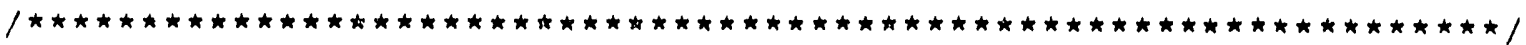

$$
\begin{aligned}
& \text { AESA[current_season] = AESA[current_season] + ESA; } \\
& \text { AENSA[current_season] = AENSA[current_season] + ENSA; }
\end{aligned}
$$

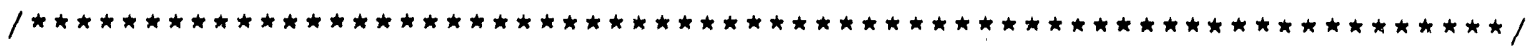
$1 *$ Use the charger model to account for fixed battery $10 s s \star /$

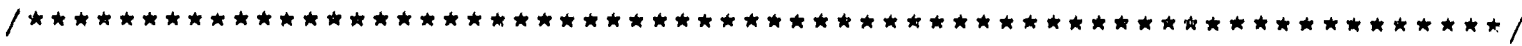

$\begin{array}{ll}\text { ESA } & =\text { model charger }(E S A, \text { el, } n 4) ;\end{array}$

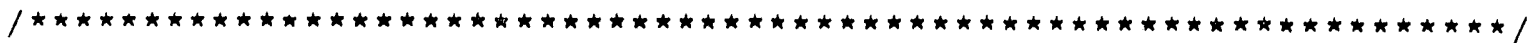

$/ *$ Compute the electricity cost for the season and keep a $\quad$ *

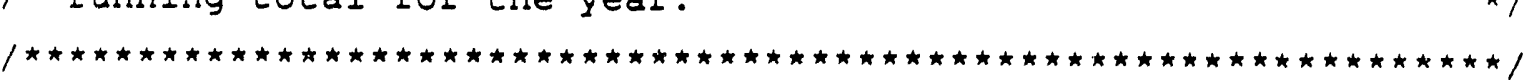

electricity_cost $S A=(E S A / 1000) * 06$

electricity-cost TNSA = (ENSA/1000)*C6;

days [current seasonT;

rent sēasonT; days [current season';

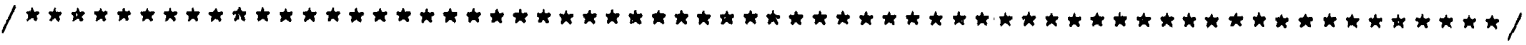

$1 *$ Calculate the depreciation of the batteries for each season $/$

/* note: The variable life SA(NSA) being used is misleading. */

/* The model returns the depreciation of the battery but */

$/ *$ must be summed and inverted at the end of the year to $/ /$

compute the battrey iffe for cost analysis.

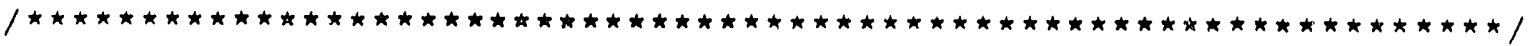

life SA[current season] = battery_dep_season(Tcal, Nfc, Ncy, xO, maxdod_SA, alpha, (double) 1.0 , days [Current season], 1 )

life NSA[current seasoñ] = battery dep season (Tcal, NfC, Ncy, X0, maxdod_NSA, aIpha, (doubleT1.0, days [current_seasōn], I):

if (maxdod SA > maxclod year SA [V type-1] [b type-1])

maxdod year SA[V Eype-1][b type-1]-= maxdod SA;

if (maxdod_SA > maxdod year NSA [V type-1] [b_type-1])

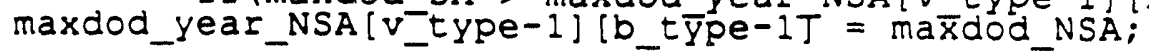

$$
\text { B-8 printed } 07 / 25 / 90 \quad 3: 20 \text { PM }
$$


.1

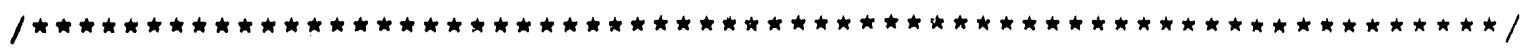

$1 /$ the data the vehicle has depleted the energy of the battery, $\quad * /$

/ are performed and the simulation aborted.

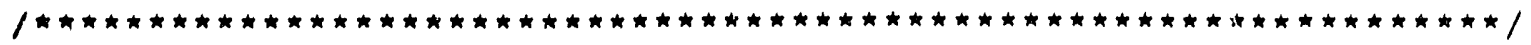

If (out_of_gas[v_type-1][b_type-1]) goto LABEL;

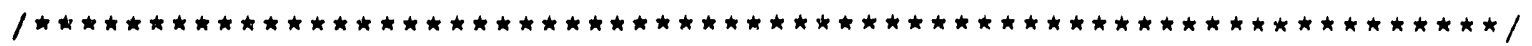

Sum the totals for the entire year to calculate yearly $/ /$
$/ *$ totals.

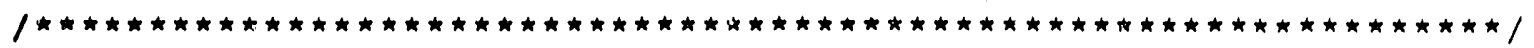

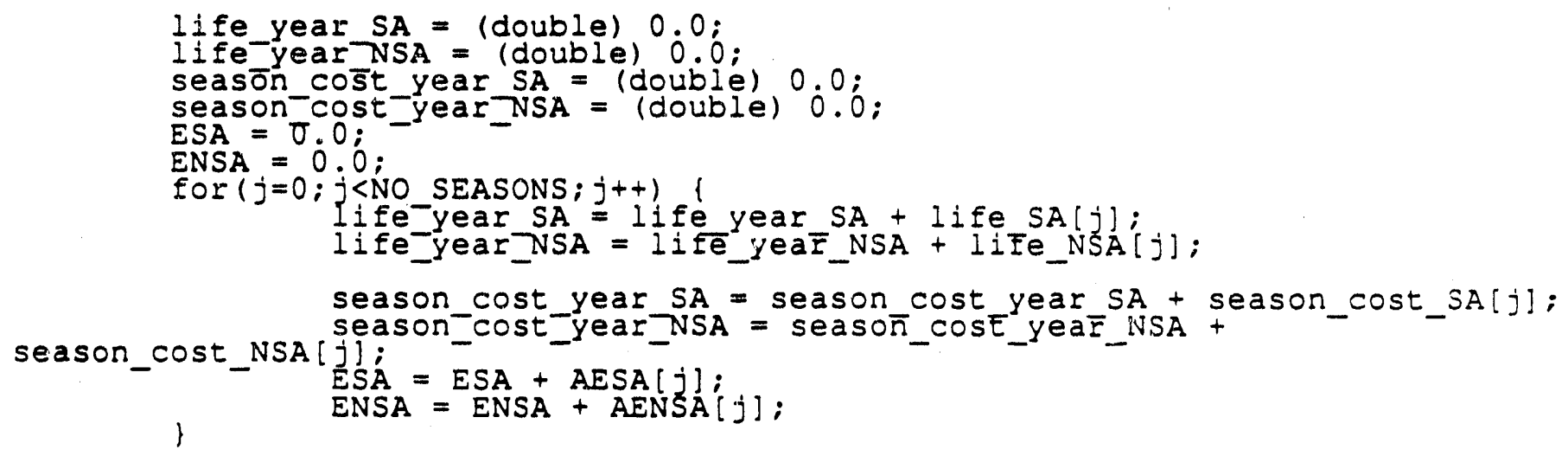

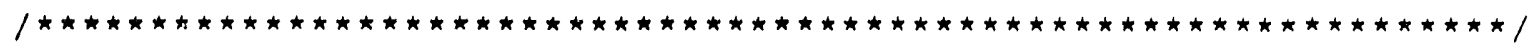

Calculate the average energy use for the year, with and $\star /$
$/$ * without the use of solar arrays.

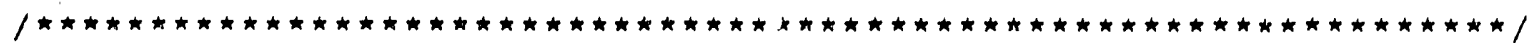

$E N S A=E N S A /$ (double) NO SEASONS;

$E S A=E S A /$ (double) NO_SEASONS;

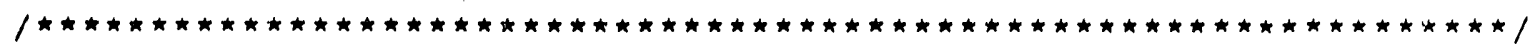

1* Compute battery life for the year. $* /$

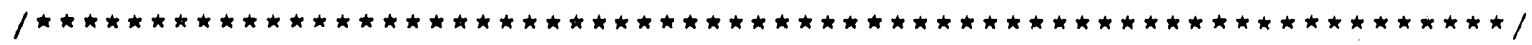

life year $S A=1 / 1$ ife year $S A$;

life_year_NSA = I/life_year_NSA;

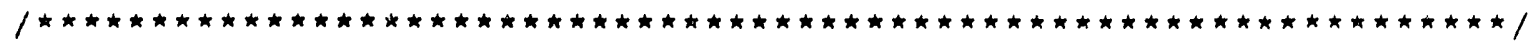

$\begin{array}{ccc}/ \star & \text { Calculate the percent difference of: } & \star / \\ / \star & \text { battery ife increase: } & \star / \\ / \star & \text { energy reduction: } & \star / \\ / \star & \text { DOD reduction: } & \star /\end{array}$

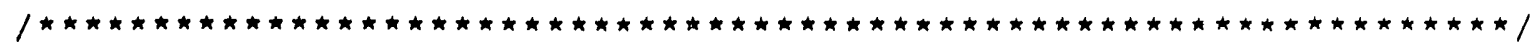

percent battery_life increase[v_type-1][b_type-1] = ( 1 ife_year_SA_life year NSA)/Iife year NSAT*100; ESA) /ENSA) $\star 100.0$; 


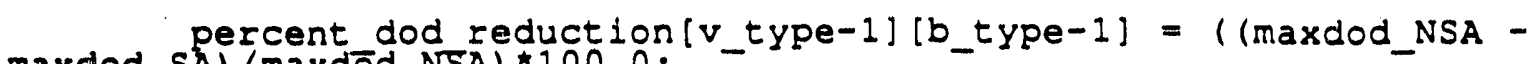
maxdod_SA) /maxdod_NSA) 100.0 ;

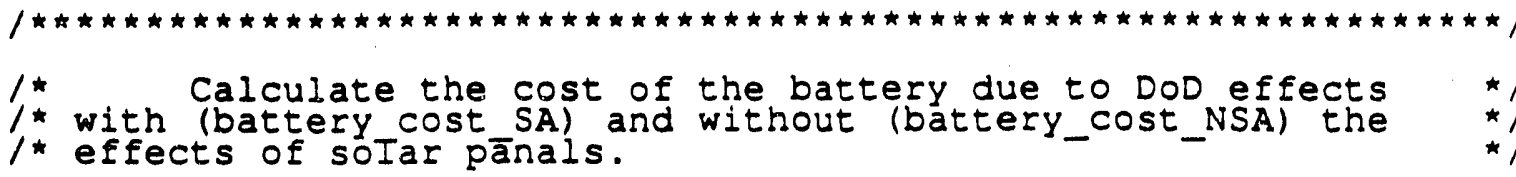

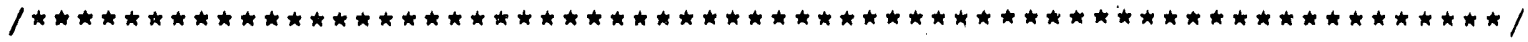

battery cost $S A=\left(c 5^{\star} e 2\right) /(1$ ife year SA):

batterY__COE_NSA $=(c 5 *$ e2) / (IIIE_year_NSA);

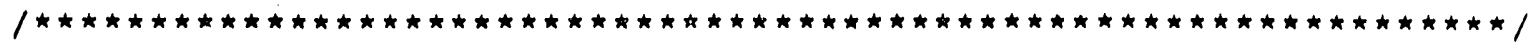

$1 *$ Compute the cost saved per year on battery life and energy
$/ *$ costs and compute the payback period.

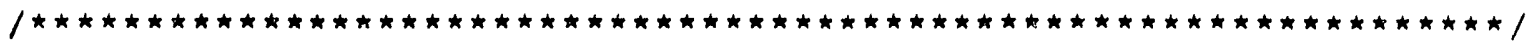

battery cost NSA[v_type-1][b_type-1]= (season_cost_year_NSA +

-cost-SA[v type-1][b type-1] = (season cost_year SA + battery cost SA);

cost-saved [v type-1T[b_type-1] = cost_NSA[v-type=1][b_type-1]- -

cost_SA[v_type-1][b_Eype-1];

LABEL: ;

upfrontcojt [v type-1] [b type-1] $=(c 1+c 2 * a r e a)+(c 3+c 4 \star a r e a \star n 2) ;$ if ( out of gasTv type-1] To type-1] ) = upfrontcost [v_type-1][b_type-1]/ cost_saved[v_type-1][b_type-1];

\}

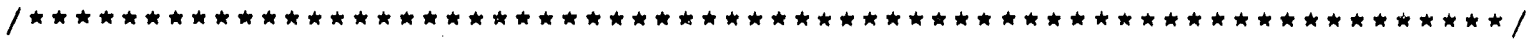

1 * Print the data $\quad$ */

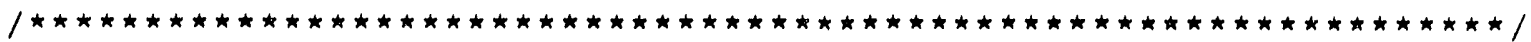

strcpy (stringl," | Up Eront Costs (\$) |");

for $(1=0 ; 1<3 ; 1++), 1<3 ; m++)$ sprintf(string2," $86.11 f \mid "$, upfrontcost [m] [1]);

\} strcat (stringl, string 2 ):

strcat (stringl, "\n");

printf ("os", stringl);

strcpy (stringl," | क energy reduction l"); for $\left(1=0 ; \quad \begin{array}{ll}1<3 ; \\ \text { for }(m=0 ; m<3 ; m++)\end{array}\right.$

if (out_of gas [m] [l])

else

sprintf(string2,"86.11f |",

percent_energy_reduction [m] [I]);

strcat ('string1, string2);

\}

\}

strcat (stringl, "\n");

printe ("os", stringl);

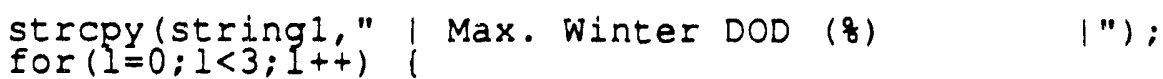

for $(1=0 ; 1<3 ; 1++) \quad$ for $(m=0 ; m<3 ; m++)$

$$
\begin{aligned}
& \text { for }(m=0 ; \underset{\text { if }}{m<3 ; m++) ~(o u t ~ o f ~ g a s ~}[m][1]) \\
& \text {-sprinte(string } 2, " \% 6.11 f(", 100.0) \text {; }
\end{aligned}
$$

printad $07 / 25 / 90$

$3: 20 \mathrm{PM}$ 


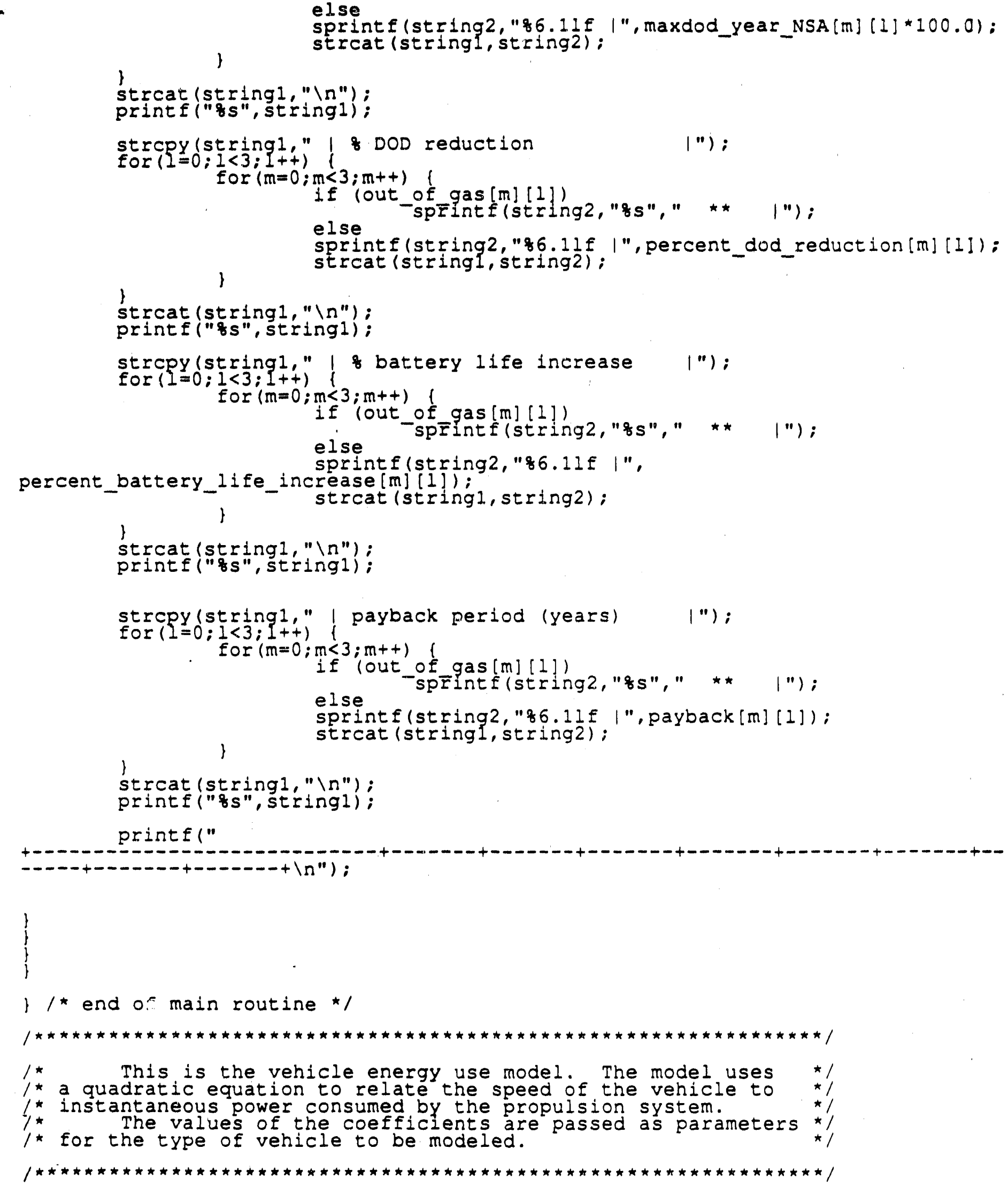


double model_vehicle_energy_use (speed, linear_term, quad_term, intercept, battery eff) double. Speed, IInear_term, quad_term, intercept, battery_eff;

double tmp;

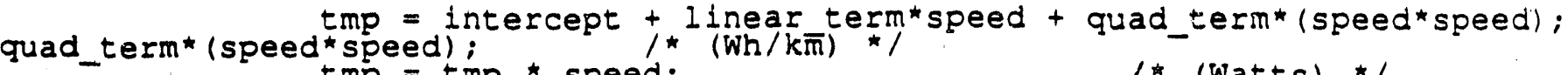

$$
\text { tmp }=\text { timp * speed; } / * \text { (Watts) * }
$$

/

$1 \star *$

efficiency

Account for blinear_termttery efficiency

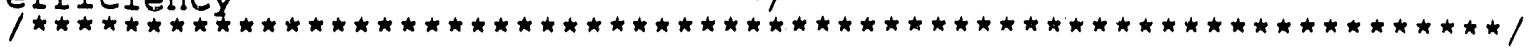

tmp = tmp / battery_eff:

return (tmp):

\}

/

/* The PV system is modeled by accounting for the PV cell */

/* efficiency and the coverter efficiency and tare loss.

/* the energy input by the PV. It is assumed that proper *

/* cicuitry exists to disable the system to avoid negative $/$ * $/$ /

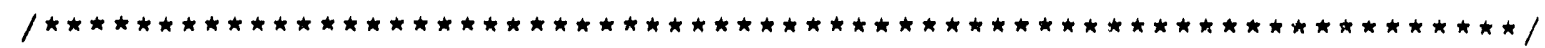

double model_PV system (input, cell eff, tare loss, convert_eff)

double input, ceIl_eff, tare_loss, cōnvert_efF;

double tmp;

tmp $=($ (input*cell_eff) -tare_loss) * convert_erf;

if $(t m p<0.0)$ tmp $=0.0$;

r return (tmp):

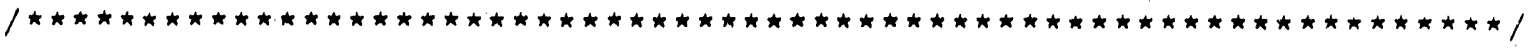

/ The charger model determines the amount of energy that must $\star /$

/ * be replaced during the charging of the batteries. The fixed */

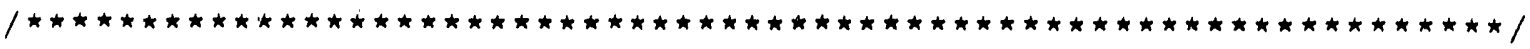

double model charger (energy, fixed_loss, charger_eff)

touble enerḡ, fixed_loss, charger-eff;

return ( (energy + fixed_loss ) / charger_eff ) ;

\}

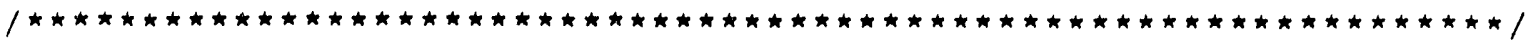

/ Routine to print the header of each page of data. */

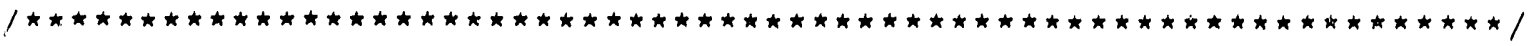

print_head ()

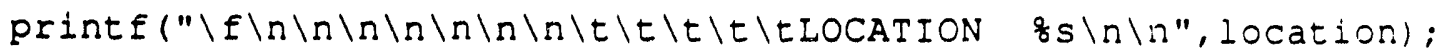

$$
\text { B-12 printed } 07 / 25 / 90 \quad 3: 20 \mathrm{PM}
$$


//kathy/ext_disk/storen/solar/prog1.c

modified 07/25/90 2:28 PM

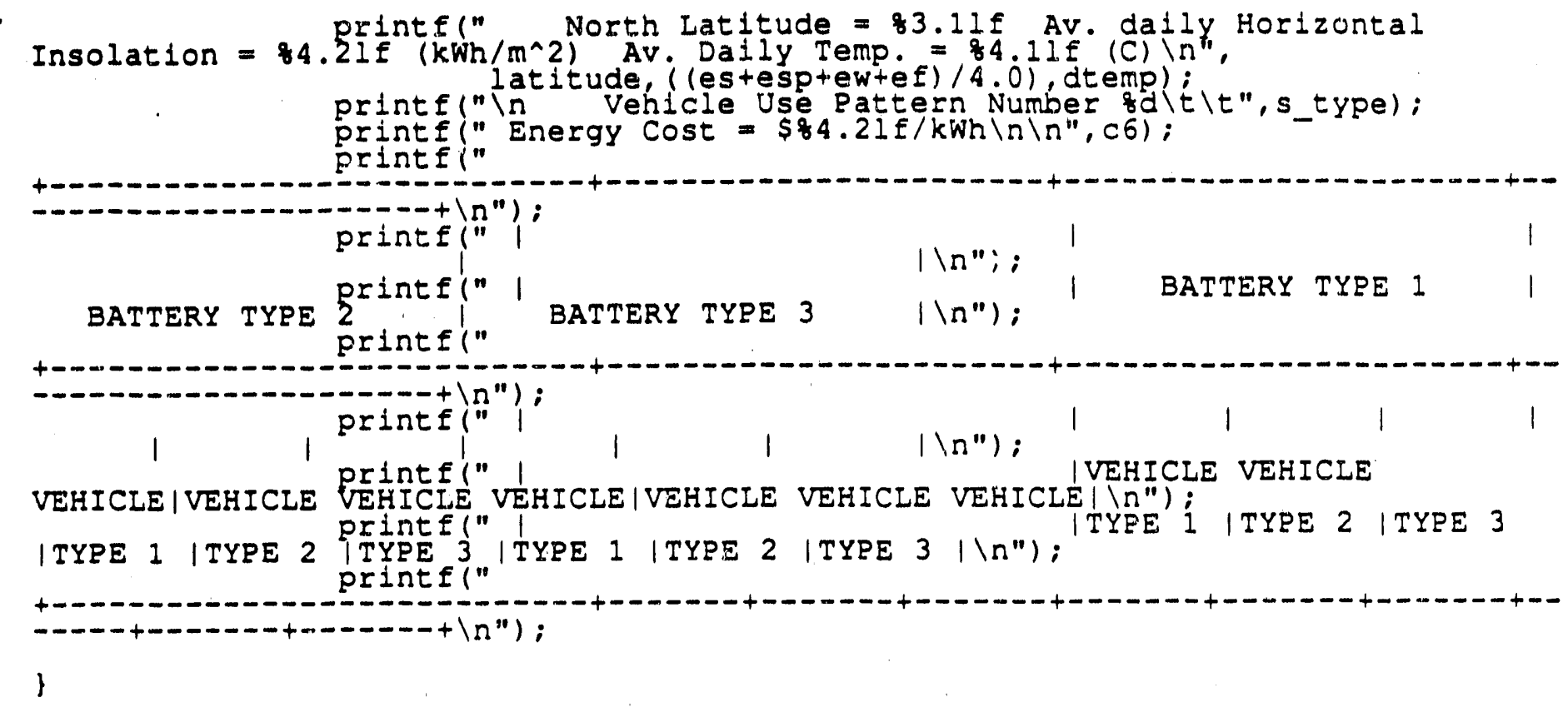




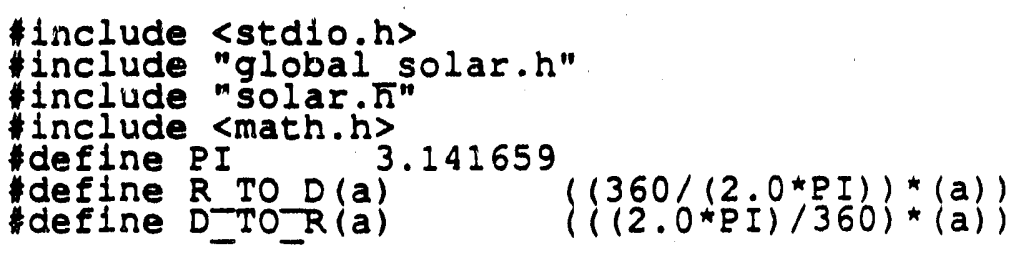

double $V(t, f l a g)$

float $t$; int flag;

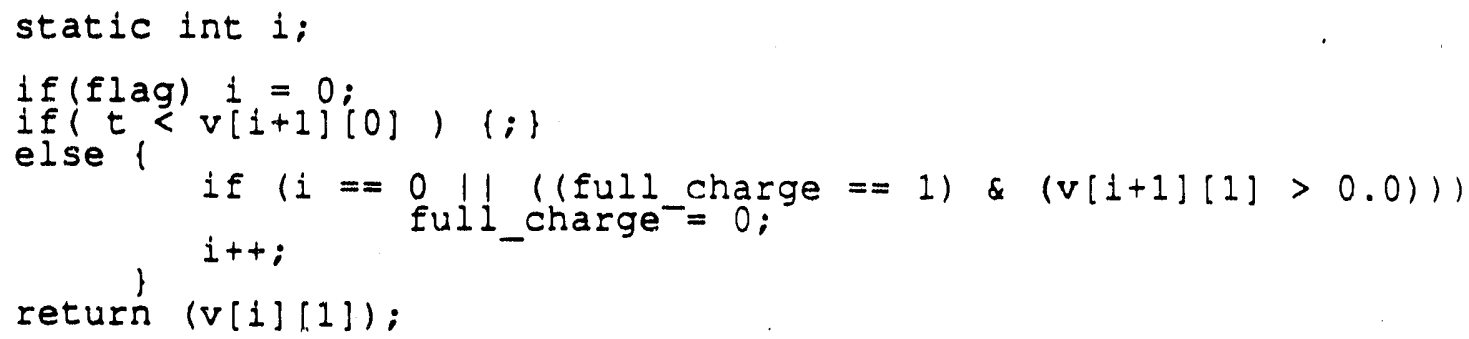


1

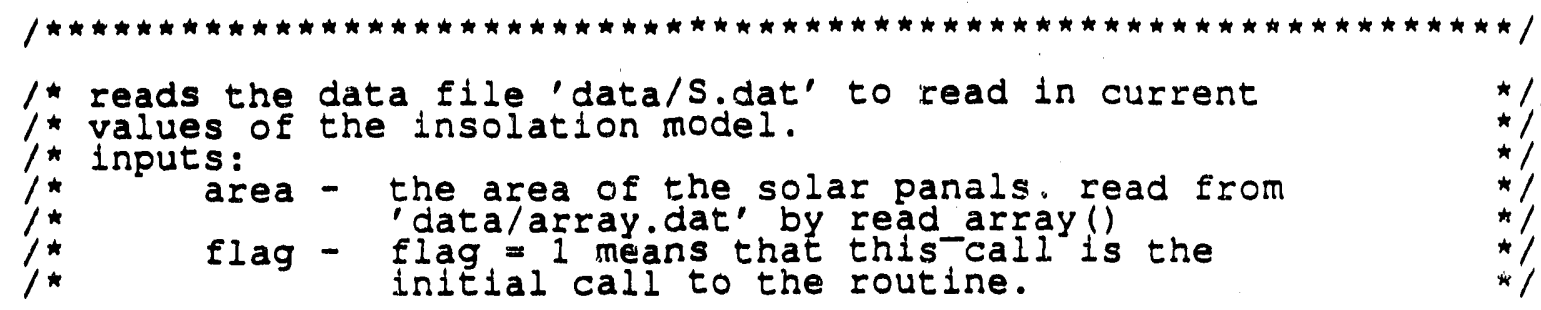

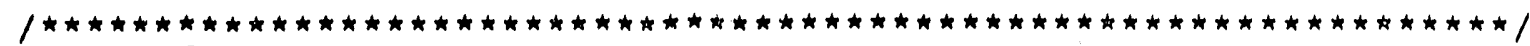
read $S(f l a g)$ int Ilag:

static EILE * $f p ;$

if(flag) ( $f p=$ fopen ("data/s.dat" "r") :
If (!fp) syserr("file not found: s_data.dat In");
return(1):

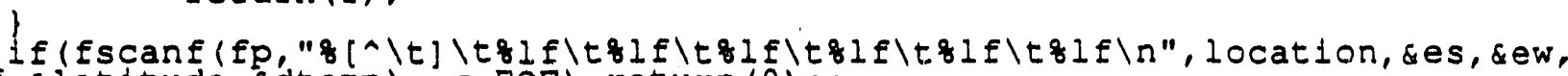
\&esp, \&ef, \&latitude, \&dtemp) $=E O E)$ return $(0) ;:$

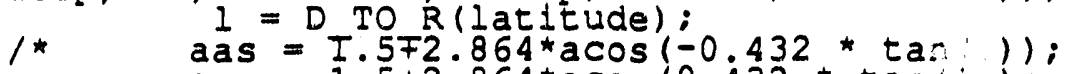

aas $=1 \cdot 5+2.864 * \operatorname{acos}(0.432 * \tan (i)$,

a asp $=6.0 ;$

$\star /$

a a $=6.0 ;$

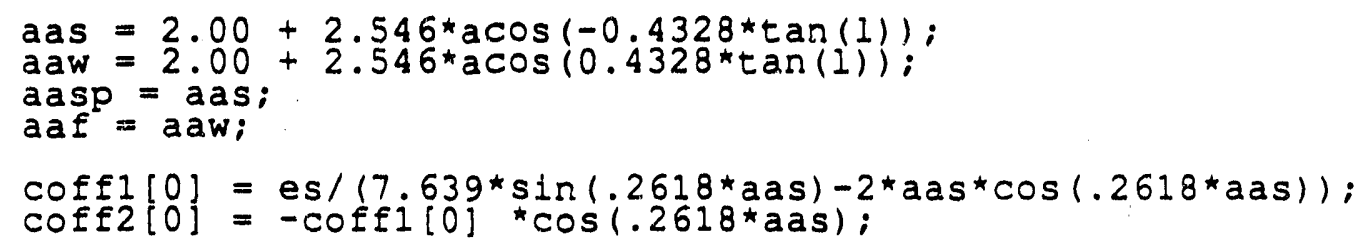

$\operatorname{coffl}[1]=\operatorname{ef} /(7.639 * \sin (.2618 * a a f)-2 * a a f * \cos (.2618 * a a f)) ;$ $\operatorname{coff} 2[1]=-\operatorname{coffl}[1] * \cos (.2618 * \operatorname{aaf}) ;$

$$
\begin{aligned}
& \left.\operatorname{coffl}[2]=e w /(7.639)^{*} \sin (.2618 \star a a w)-2 \star a a w^{\star} \cos (.2618 \star a a w)\right) \text {; } \\
& \operatorname{coff} 2[2]=-\operatorname{coff}[[2] \star \cos (.2618 \star \mathrm{aaw}) ; \\
& \operatorname{coff} 1[3]=\operatorname{esp} /(7.639 * \sin (.2618 * \operatorname{aasp})-2 \star \operatorname{aas} \star \cos (.2618 * \operatorname{arsp})) ; \\
& \operatorname{coff}[3]=-\operatorname{coffl}[3] \star \cos (.2618 \star \operatorname{aasp}) ;
\end{aligned}
$$

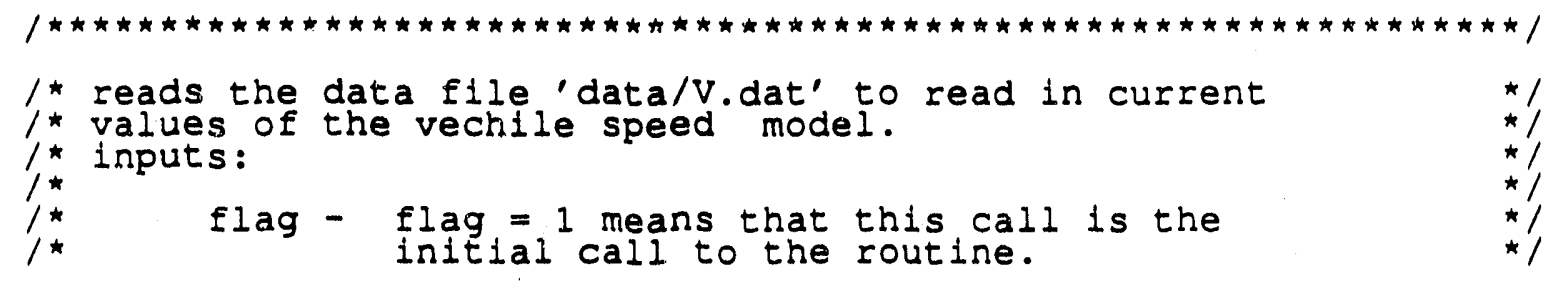

int read $V(f l a g)$

int flagi

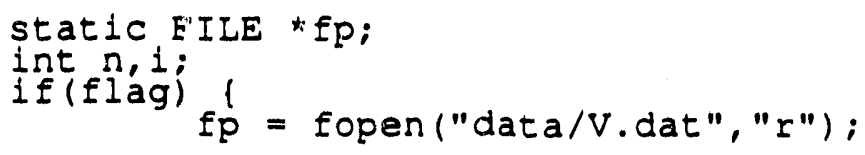


if (fscanf'fp, "8d", \&n)=z EOE) return $(0)$;

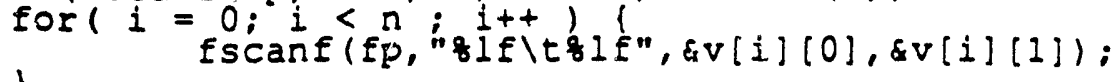

I setype++;
return (n);

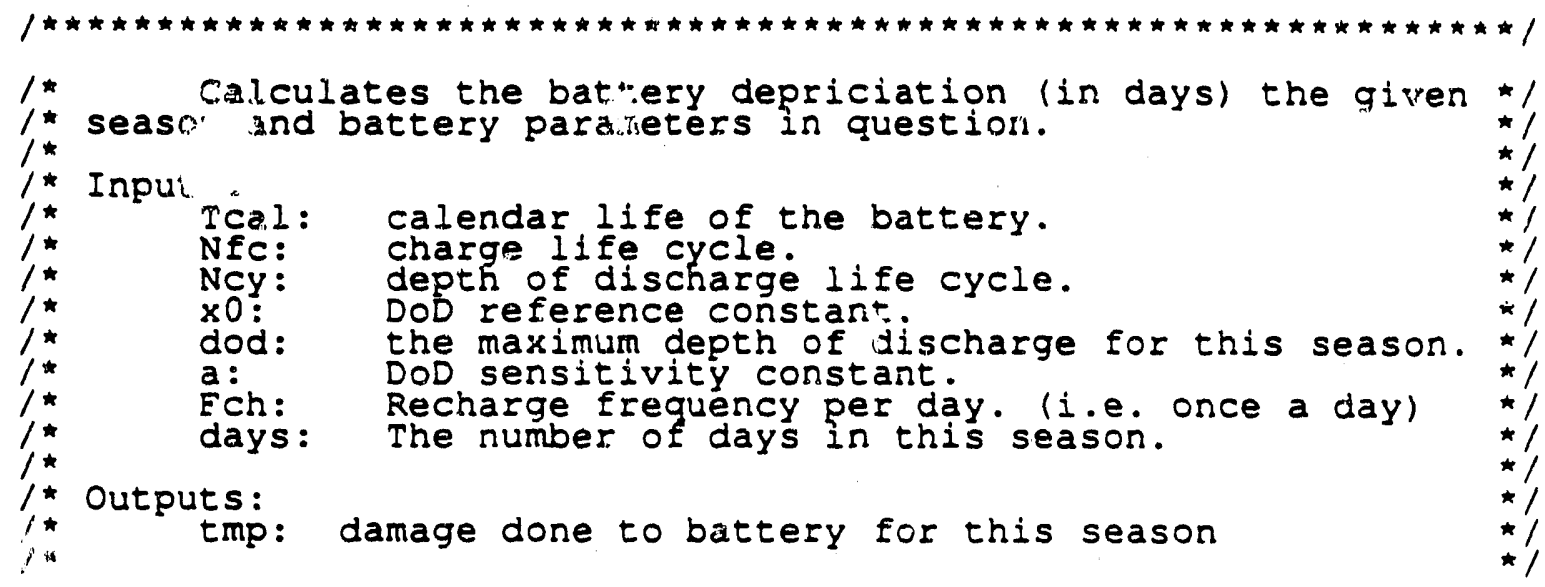

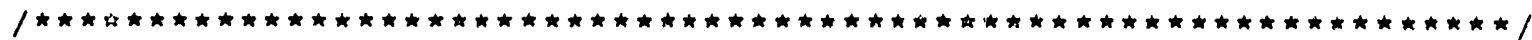

double battery dep season (Tcal, Nfc, Ncy, xO, dod, a, Fch, days, flag) double dod, TCaI, NEC, NCY, a, Ech, xO, days; int flag;

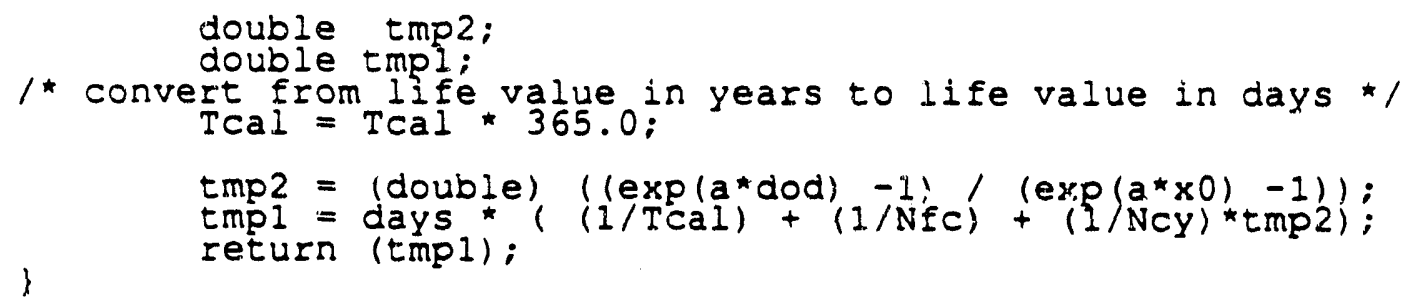

/* This function reacis the values of the array parameters.

$1 *$ Inpurs:

$1 *$ *

$1 *$

outputs:

fiag: indicates initial entry into the procedure.

$1 *$

returns:

1 when valid data is read in

0 when EOF is detected

\section{$1 *$}

read array (flag)

int Elag;

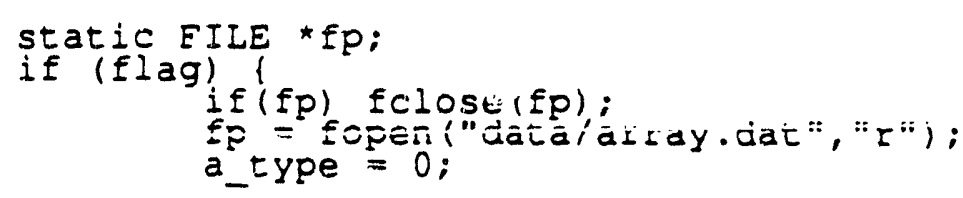


\}

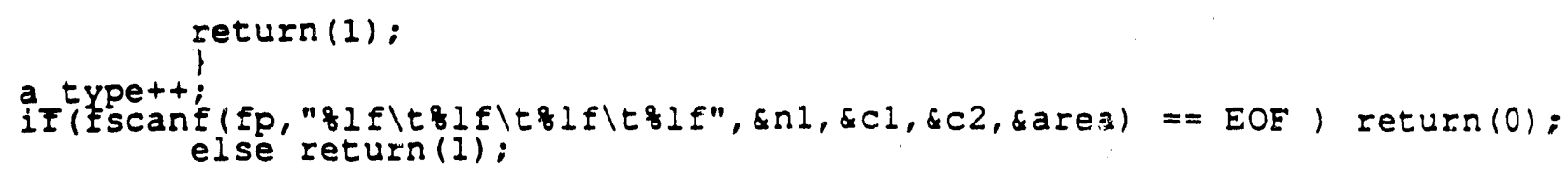

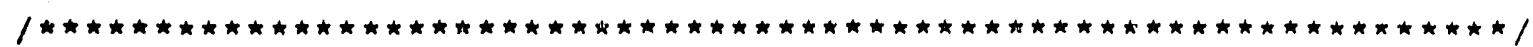

read convert (flag)

int Flag:

\}

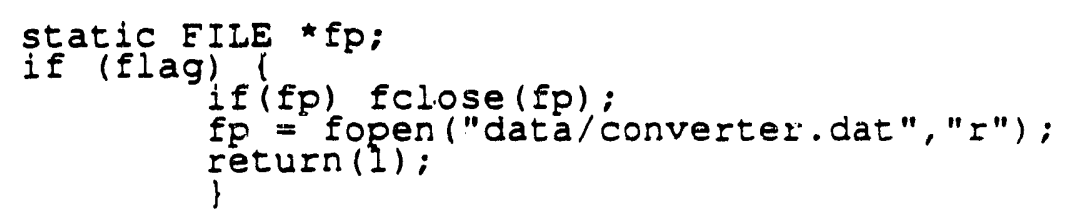

if (fscanf $(f p, " z l f \backslash t z l f \backslash t z l f \backslash t z l f ", \& p 0, \& n 2, \& c 3, \& c 4)==E O E$, return $(0)$; else return(1):

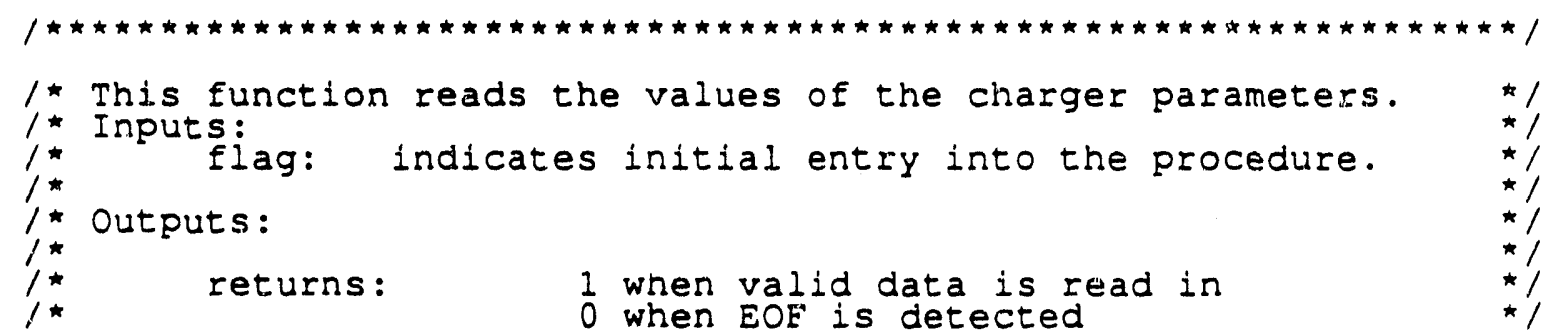

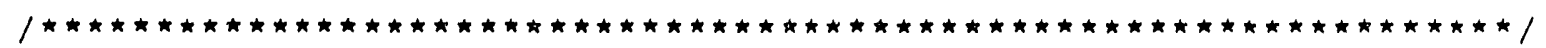

read charger(flag)

int Ilag;

\}

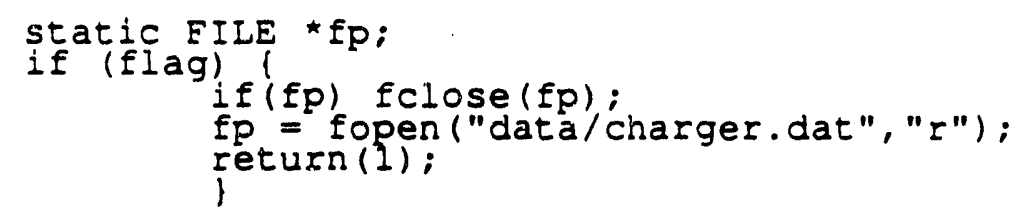

if $(f \operatorname{scanf}(f p, " q I f ", \& n 4)==E O F$, return $(0)$; else return (1);

/

1* This function reads the values of the vehicle parameters.

1 * Inputs:

$1 *$

7 *

fiag: indicates initial entry into the procedure.

outputs:

$1 *$

i*

returns:

1 when valid data is read in

0 when EOF is detected 


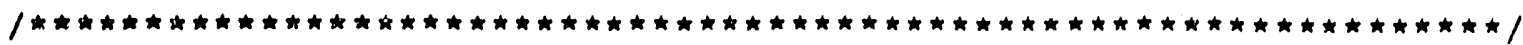

read vehlcle (flag)

int Flag;

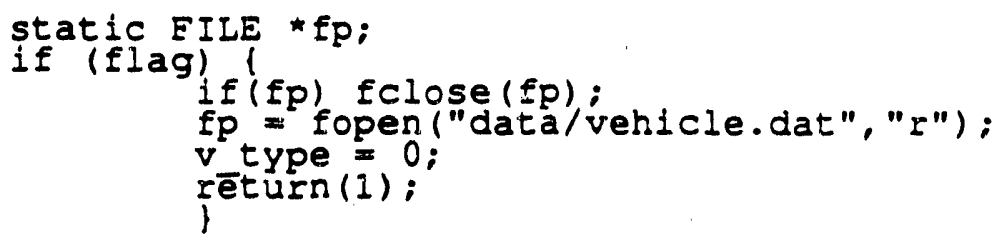

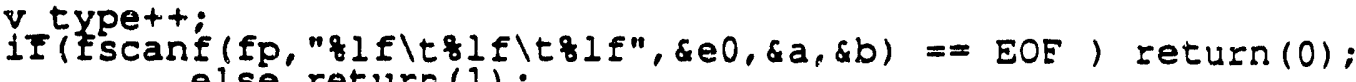 else return(1);}

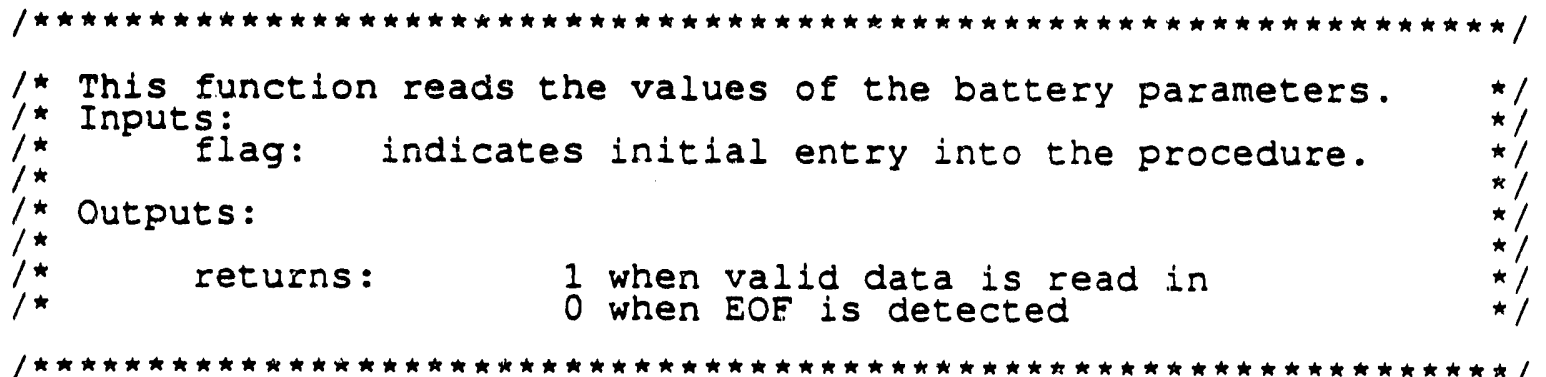

read battery (tIag)

int Flag;

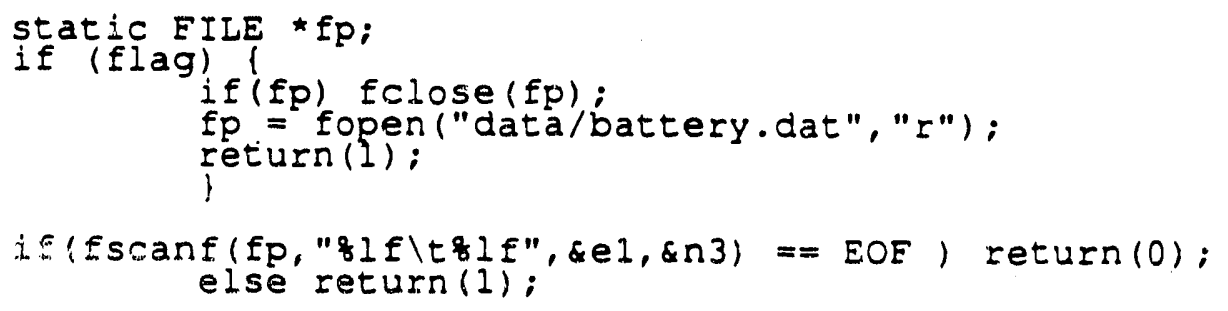

1 * This function reads the values of the battery 1 ife

/* parameters.

$1 \cdot *$ Inputs:

$\frac{1}{1}$ outputs:

returns:

1 when valid data is read in

0 when EOF is detected

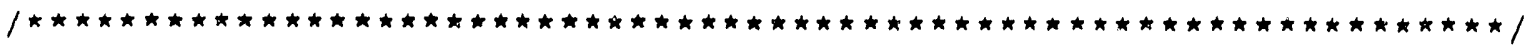

read battery_life(flag)

int Ilag;

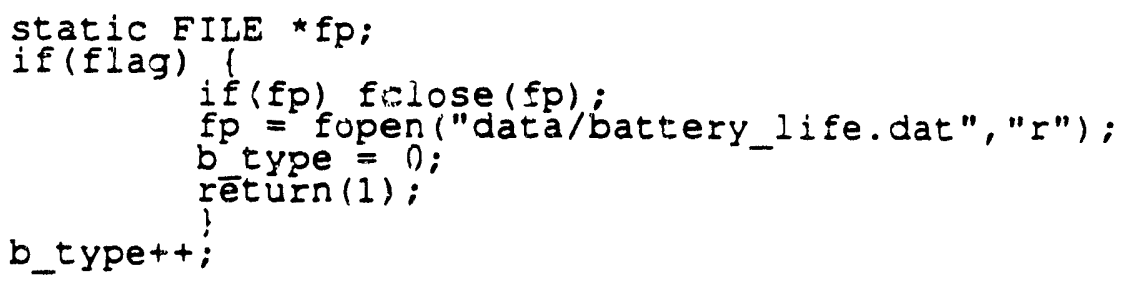




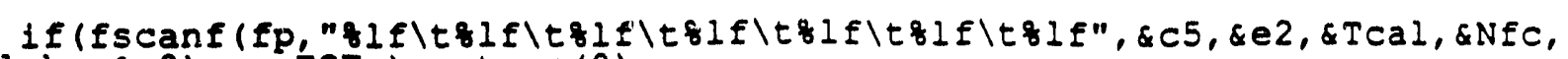

- ENCY, \&alpha, \&XO) =EOF, return $(0)$;

)

else return(1):

/

1 * This function reads the values of the cost parameters.

/* Inputs

fiag: indicates initial entry into the procedure.

* Outputs:

$1 *$

returns:

1 when valid data is read in

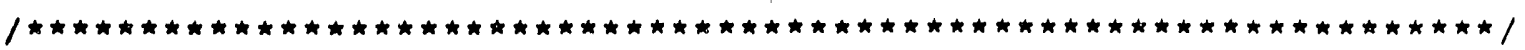

read cost (flag)

int Ilag:

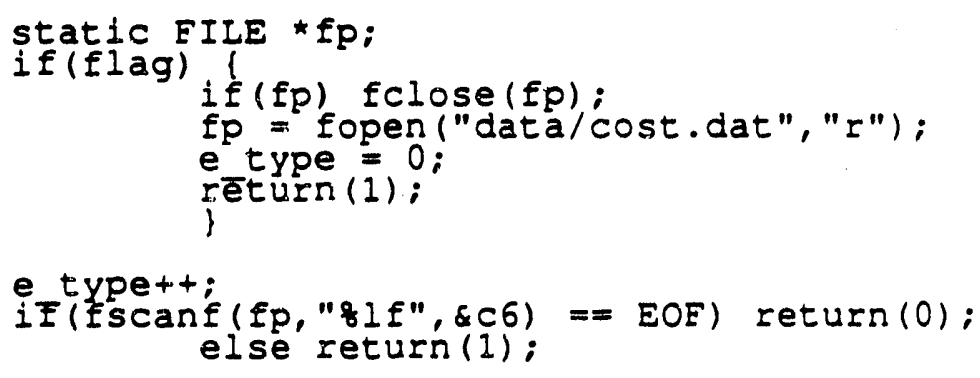


/

1* This routine is used to produce error messages

$\downarrow /$

$1 *$

include <stalo.h>

include <errno.h>

vold syseri (msg)

char *msg:

cha

extern int errno, sys nerr:

extern char "sys erIIst[];

fprinte (stderr, "ERROR: ofs (" od", msg, errno):

if (errno > 0 \& errno < sys nerr)

printf(") os",sys_errlist errnoj):

$\operatorname{expt}(1)$

else fprinte(stderr,") $\backslash \Omega$ "):

) 

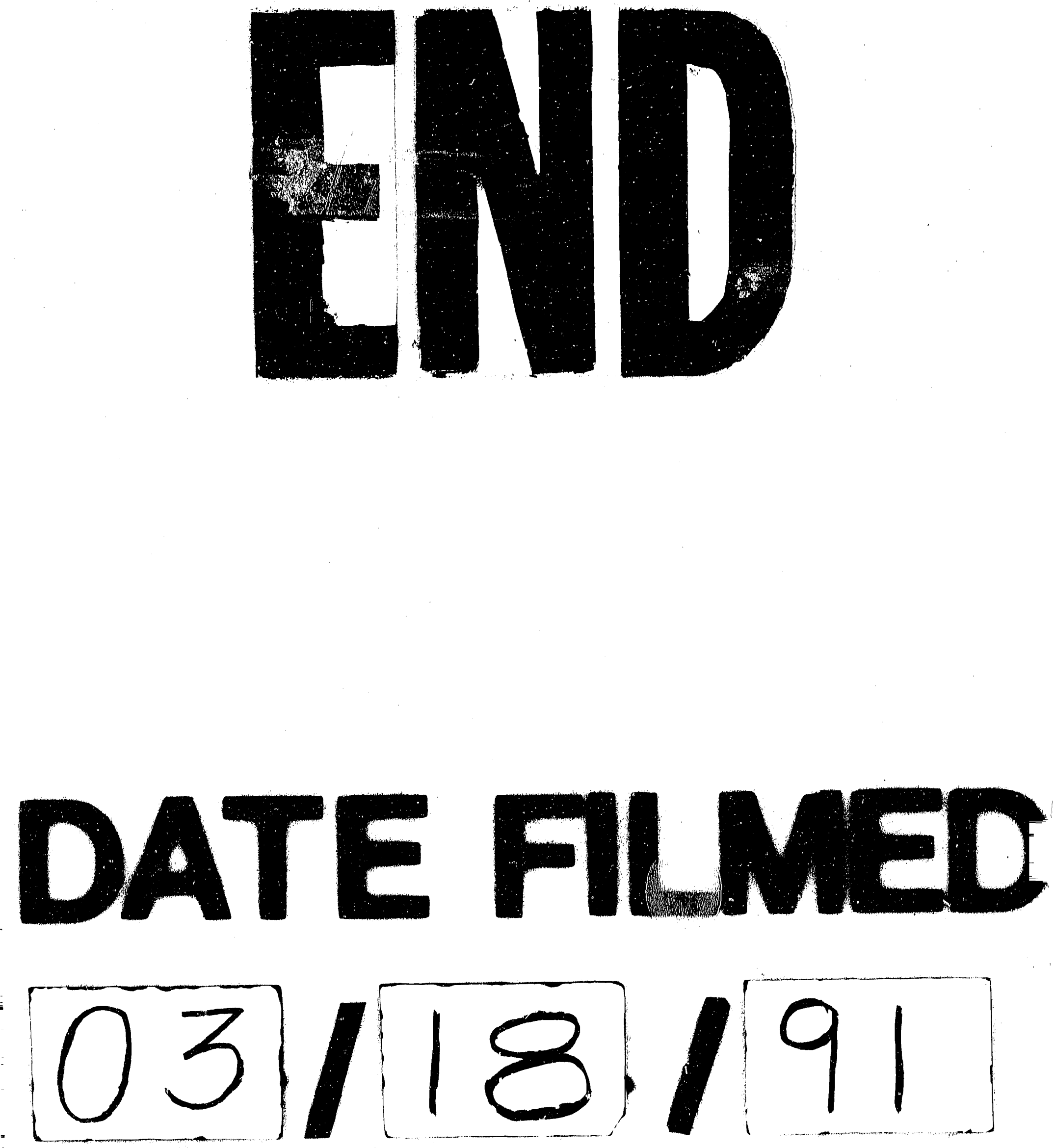
nt. . . w 